\title{
Chiral Ferrocenyl P,N-Ligands for Palladium-Catalyzed Asymmetric Formal [3+2] Cycloaddition of Propargylic Esters with $\beta$-Ketoesters: Access to Functionalized Chiral 2,3-Dihydrofurans
}

Yong Zhou, Fu-Lin Zhu, Zhen-Ting Liu, Xiao-Mao Zhou, ${ }^{*}$ and Xiang-Ping Hu*

Biotechnology Research Center, Hunan Academy of Agricultural Sciences, Changsha, China, and Dalian Institute of Chemical Physics, Chinese Academy of Sciences, Dalian 116023, China xiangping@dicp.ac.cn; zhouxm1972@126.com

\section{Supporting Information}

General Information. S2

General Procedure for Preparation of Chiral Ligands $\left(R_{c}, S_{p}\right)-L 4 \ldots \ldots \ldots \ldots \ldots . . . . . .22$

General Procedure for Pd-Catalyzed Asymmetric [3+2] Cycloaddition of Propargylic Esters with $\beta$-Ketoesters. .54

Transformation of Cycloadduct 3aa. S30

References. S32

Crystal Data and Structure Refinement for $\left(\boldsymbol{R}_{c}, \boldsymbol{S}_{p}\right)$-L4a.EtOH.....................33

Crystal Data and Structure Refinement for (S)-3ej. .534

NMR Spectra. S36 


\section{General Information}

All reactions were carried out under a nitrogen atmosphere. Solvents were purified by standard procedure before use. Commercial reagents were used without further purification. Flash chromatography was performed on silica gel $60(40-63 \mu \mathrm{m}, 60 \AA)$. Thin layer chromatography (TLC) was performed on glass plates coated with silica gel 60 with F254 indicator. Proton nuclear magnetic resonance ( ${ }^{1} \mathrm{H}$ NMR) spectra were recorded on a Bruker $400 \mathrm{MHz}$ spectrometer. Chemical shifts for protons are reported in parts per million downfield from tetramethylsilane and are referenced to residual protium in the NMR solvent $\left(\mathrm{CHCl}_{3}=\delta 7.28\right)$. Carbon nuclear magnetic resonance $\left({ }^{13} \mathrm{C}\right.$ NMR) spectra were recorded on a Bruker $100 \mathrm{MHz}$ spectrometer. Chemical shifts for carbon are reported in parts per million downfield from tetramethylsilane and are referenced to the carbon resonances of the solvent $\left(\mathrm{CDCl}_{3}=\delta 77.07\right)$. Data are represented as follows: chemical shift, multiplicity ( $b r=$ broad, $s=$ singlet, $d=$ doublet, $t=$ triplet, $q=$ quartet, $m=$ multiplet), coupling constants in Hertz $(\mathrm{Hz})$, integration. Infrared spectra were recorded on a Bruker FTIR spectrometer equipped with an ATR and are reported in reciprocal centimeters $\left(\mathrm{cm}^{-1}\right)$. Only the most important and relevant frequencies are reported. Enantiomeric ratios were determined by chiral HPLC with hexane and $i-\mathrm{PrOH}$ as solvents. Optical rotations were recorded on a JASCO $\mathrm{P}-1020$ polarimeter. $\beta$-Ketoesters $\mathbf{1}^{1}$ and propargylic esters $2^{2}$ were prepared following the method from the literature.

\section{General Procedure for Preparation of Chiral Ligands $\left(\boldsymbol{R}_{c}, S_{p}\right)-L 4^{3}$}
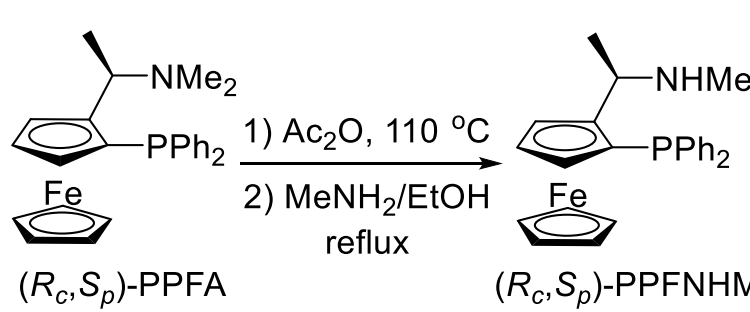

$\left(R_{c}, S_{p}\right)$-PPFNHMe

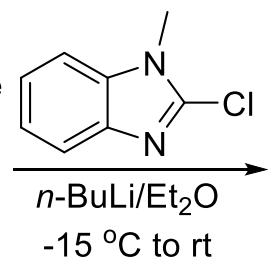

$-15^{\circ} \mathrm{C}$ to rt

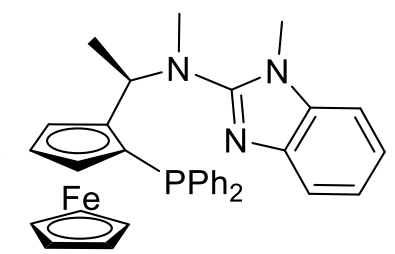

$\left(R_{c}, S_{p}\right)-\mathbf{L} \mathbf{a}$

To a solution of $\left(R_{c}, S_{p}\right)$-PPFNHMe $(1.71 \mathrm{~g}, 4.0 \mathrm{mmol})$ in $10 \mathrm{~mL}$ of Et $2 \mathrm{O}$ was added $n$-BuLi (1.6 $\mathrm{mL}, 4.4 \mathrm{mmol}$ ) at $-15^{\circ} \mathrm{C}$ under a nitrogen atmosphere. The reaction mixture was stirred $30 \mathrm{~min}$ at the same temperature, and then a solution of 2-chloro-1-methyl- $1 \mathrm{H}$-benzo[d]imidazole $(0.66 \mathrm{~g}, 4$ $\mathrm{mmol}$ ) in $10 \mathrm{~mL}$ of $\mathrm{Et}_{2} \mathrm{O}$ was added dropwise to the reaction mixture. The resulting solution was allowed to warm to room temperature after stirring for $30 \mathrm{~min}$. After stirring at room temperature 
for $12 \mathrm{~h}$, the reaction mixture was quenched with saturated aqueous ammonium chloride, then extracted with ethyl acetate $(3 \times 20 \mathrm{~mL})$, washed with brine $(1 \times 10 \mathrm{~mL})$, dried over anhydrous sodium sulfate, and concentrated in vacuo. The residue was purified by column chromatography on silica gel to give $\left(R_{c}, S_{p}\right)$-L4a $(1.90 \mathrm{~g}, 85 \%$ yield $)$ as an orange solid. M.p.: $198-200{ }^{\circ} \mathrm{C}$. $[\alpha]_{D^{21}}=$ -411.1 (c 1.15, $\left.\mathrm{CH}_{2} \mathrm{Cl}_{2}\right) .{ }^{1} \mathrm{H}$ NMR $\left(400 \mathrm{MHz}, \mathrm{CDCl}_{3}\right): \delta$ 7.57-6.96 (m, 14H), 5.45 (dd, $J=6.6,2.3$ $\mathrm{Hz}, 1 \mathrm{H}), 4.58(\mathrm{~s}, 1 \mathrm{H}), 4.40(\mathrm{~s}, 1 \mathrm{H}), 3.97(\mathrm{~s}, 6 \mathrm{H}), 2.82(\mathrm{~s}, 3 \mathrm{H}), 2.44(\mathrm{~s}, 3 \mathrm{H}), 1.73(\mathrm{~d}, J=6.7 \mathrm{~Hz}, 3 \mathrm{H})$; ${ }^{13} \mathrm{C}$ NMR $\left(101 \mathrm{MHz}, \mathrm{CDCl}_{3}\right): \delta 158.2,141.9,140.9(\mathrm{~d}, J=9.9 \mathrm{~Hz}), 138.2(\mathrm{~d}, J=9.4 \mathrm{~Hz}), 136.2$, 135.4 , 135.2, 132.4, 132.2, 129.1, 128.1, 128.0, 127.9, 127.9, 127.7, 121.1, 120.0, 117.2, 107.9, $94.7(\mathrm{~d}, J=26.1 \mathrm{~Hz}), 76.1$ (d, $J=11.2 \mathrm{~Hz}), 72.3(\mathrm{~d}, J=5.0 \mathrm{~Hz}), 70.5(\mathrm{~d}, J=4.4 \mathrm{~Hz}$ ), 69.9, 69.4, $54.1(\mathrm{~d}, J=8.1 \mathrm{~Hz}), 32.3,30.6(\mathrm{~d}, J=5.1 \mathrm{~Hz}), 13.9 ;{ }^{31} \mathrm{P} \mathrm{NMR}\left(162 \mathrm{MHz}, \mathrm{CDCl}_{3}\right): \delta-26.5$. HRMS calc. for $\mathrm{C}_{33} \mathrm{H}_{33} \mathrm{FeN} \mathrm{N}_{3} \mathrm{P}[\mathrm{M}+\mathrm{H}]^{+}:$558.1762, found: 558.1741 .

In a similar procedure, $\left(R_{c}, S_{p}\right)$-L4b-d was prepared as follows:

$\left(R_{c}, S_{p}\right)$-L4b: Orange solid (1.87g, 82\% yield). M.p.: 218-220 ${ }^{\circ} \mathrm{C} .[\alpha]_{\mathrm{D}}{ }^{18}=-369.9\left(c 1.00, \mathrm{CH}_{2} \mathrm{Cl}_{2}\right)$.

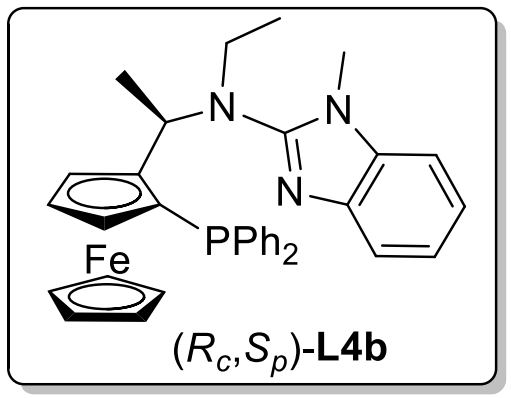
${ }^{1} \mathrm{H}$ NMR $\left(400 \mathrm{MHz}, \mathrm{CDCl}_{3}\right): \delta$ 7.60-7.49 $(\mathrm{m}, 3 \mathrm{H}), 7.34-7.33(\mathrm{~m}, 3 \mathrm{H})$, 7.16-7.01 (m, 8H), 5.27-5.25 (m, 1H), $4.59(\mathrm{~s}, 1 \mathrm{H}), 4.39(\mathrm{~s}, 1 \mathrm{H})$, $3.99(\mathrm{~s}, 1 \mathrm{H}), 3.94(\mathrm{~s}, 5 \mathrm{H}), 3.14(\mathrm{dq}, J=14.2,7.0 \mathrm{~Hz}, 1 \mathrm{H}), 2.94(\mathrm{~s}$, $3 \mathrm{H}$ ), 2.87 (dq, $J=14.0,7.0 \mathrm{~Hz}, 2 \mathrm{H}), 1.74(\mathrm{~d}, J=6.8 \mathrm{~Hz}, 3 \mathrm{H}), 0.70$ (t, $J=7.1 \mathrm{~Hz}, 3 \mathrm{H}) ;{ }^{13} \mathrm{C} \mathrm{NMR}\left(101 \mathrm{MHz}, \mathrm{CDCl}_{3}\right): \delta 156.5,141.9,140.8$ (d, $J=9.8 \mathrm{~Hz}$ ), $138.2(\mathrm{~d}, J=9.3 \mathrm{~Hz}), 135.7,135.4,135.2,132.5$, 132.3, 129.1, 128.1, 128.0, 128.0, 127.9, 127.7, 121.1, 120.1, 117.5, 95.6 (d, $J=26.2 \mathrm{~Hz}$ ), 75.7 (d, $J=11.2 \mathrm{~Hz}$ ), $72.2(\mathrm{~d}, J=5.0 \mathrm{~Hz}$ ), $70.7(\mathrm{~d}, J=4.5 \mathrm{~Hz}), 69.9,69.4,55.5(\mathrm{~d}, J=7.6 \mathrm{~Hz}), 39.6,30.1$ $(\mathrm{d}, J=7.2 \mathrm{~Hz}), 16.1,14.1 ;{ }^{31} \mathrm{P}$ NMR $\left(162 \mathrm{MHz}, \mathrm{CDCl}_{3}\right): \delta$-26.3. HRMS calc. for $\mathrm{C}_{34} \mathrm{H}_{35} \mathrm{FeN}_{3} \mathrm{P}$ $[\mathrm{M}+\mathrm{H}]^{+}:$572.1918, found: 572.1874 .

$\left(R_{c}, S_{p}\right)$-L4c: Orange solid (1.34g, 53\% yield). M.p.: $60-62{ }^{\circ} \mathrm{C} .[\alpha]_{\mathrm{D}}{ }^{18}=-301.6\left(c 1.20, \mathrm{CH}_{2} \mathrm{Cl}_{2}\right) .{ }^{1} \mathrm{H}$

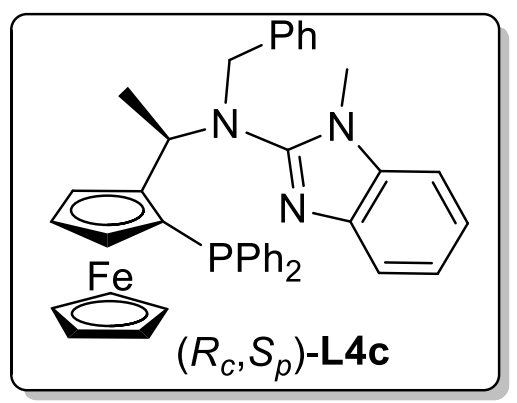

NMR $\left(400 \mathrm{MHz}, \mathrm{CDCl}_{3}\right): \delta$ 7.58-7.53 $(\mathrm{m}, 3 \mathrm{H}), 7.36-7.34(\mathrm{~m}, 3 \mathrm{H})$, 7.23-6.84 (m, 13H), 5.32-5.31 (m, 1H), 4.54-4.53 (m, 1H), $4.41(\mathrm{t}, J$ $=2.4 \mathrm{~Hz}, 1 \mathrm{H}), 4.34(\mathrm{~d}, J=15.4 \mathrm{~Hz}, 1 \mathrm{H}), 4.26(\mathrm{~d}, J=15.4 \mathrm{~Hz}, 1 \mathrm{H})$, 4.09 (s, 1H), $3.92(\mathrm{~s}, 5 \mathrm{H}), 2.86(\mathrm{~s}, 3 \mathrm{H}), 1.80(\mathrm{~d}, J=6.8 \mathrm{~Hz}, 3 \mathrm{H}) ;{ }^{13} \mathrm{C}$ $\operatorname{NMR}\left(101 \mathrm{MHz}, \mathrm{CDCl}_{3}\right): \delta 156.1,141.8,140.4(\mathrm{~d}, J=9.6 \mathrm{~Hz})$, $139.3,138.2(d, J=8.9 \mathrm{~Hz}), 135.4,135.2,132.5,132.4,129.2$, 
128.1, 128.1, 128.0, 127.8, 127.5, 126.5, 121.1, 120.2, 117.7, 108.2, 95.9 (d, J=30.7 Hz), 75.4 (d, $J=11.4 \mathrm{~Hz}$ ), $72.1(\mathrm{~d}, J=4.8 \mathrm{~Hz}), 70.6(\mathrm{~d}, J=4.6 \mathrm{~Hz}), 70.0,69.7,56.0(\mathrm{~d}, J=8.5 \mathrm{~Hz}), 50.1,30.0$ (d, $J=5.9 \mathrm{~Hz}), 17.3 ;{ }^{31} \mathrm{P}$ NMR (162 MHz, $\left.\mathrm{CDCl}_{3}\right): \delta-26.8$. HRMS calc. for $\mathrm{C}_{39} \mathrm{H}_{37} \mathrm{FeN}_{3} \mathrm{P}[\mathrm{M}+\mathrm{H}]^{+}$: 634.2075, found: 634.2106 .

$\left(R_{c}, S_{p}\right)$-L4d: Orange solid (2.03g, 80\% yield). M.p.: $230-232^{\circ} \mathrm{C} .[\alpha]_{\mathrm{D}}{ }^{18}=-262.2\left(c 0.55, \mathrm{CH}_{2} \mathrm{Cl}_{2}\right)$.

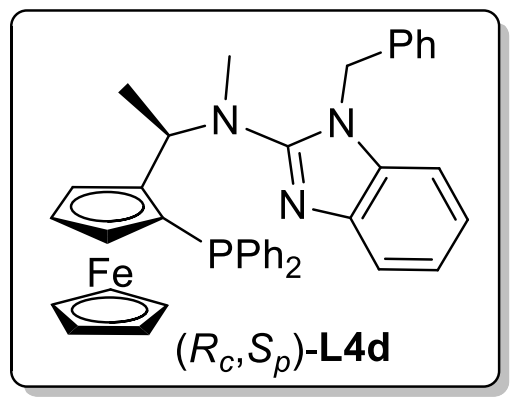

${ }^{1} \mathrm{H} \mathrm{NMR}\left(400 \mathrm{MHz}, \mathrm{CDCl}_{3}\right): \delta 7.61-6.93(\mathrm{~m}, 19 \mathrm{H}), 5.62(\mathrm{dd}, J=17.9$, $5.3 \mathrm{~Hz}, 1 \mathrm{H}), 5.18(\mathrm{~d}, J=5.7 \mathrm{~Hz}, 1 \mathrm{H}), 4.51(\mathrm{~d}, J=18.0 \mathrm{~Hz}, 1 \mathrm{H}), 4.44$ (s, 1H), $4.32(\mathrm{~s}, 1 \mathrm{H}), 3.94(\mathrm{~s}, 1 \mathrm{H}), 3.71(\mathrm{~s}, 5 \mathrm{H}), 2.23(\mathrm{~s}, 3 \mathrm{H}), 1.41(\mathrm{~d}$, $J=6.2 \mathrm{~Hz}, 3 \mathrm{H}) ;{ }^{13} \mathrm{C}$ NMR $\left(101 \mathrm{MHz}, \mathrm{CDCl}_{3}\right): \delta 158.3,141.9,140.2$ (d, $J=8.9 \mathrm{~Hz}), 137.9$ (d, $J=8.9 \mathrm{~Hz}), 137.2,136.7,135.1,134.9$, $132.7,132.5,129.1,128.7,128.1,128.1,127.2,125.8,121.7$, 120.6, 117.3, 108.7, 93.4 (d, $J=26.2 \mathrm{~Hz}), 76.3(\mathrm{~d}, J=10.8 \mathrm{~Hz}), 72.5$ (d, $J=4.6 \mathrm{~Hz}$ ), 70.6 (d, $J=$ $4.4 \mathrm{~Hz}$ ), 69.9, 69.5, 55.1 (d, $J=5.7 \mathrm{~Hz}), 47.5$ (d, $J=14.1 \mathrm{~Hz}), 32.2,13.4 ;{ }^{31} \mathrm{P}$ NMR $(162 \mathrm{MHz}$, $\left.\mathrm{CDCl}_{3}\right): \delta$-26.2. HRMS calc. for $\mathrm{C}_{39} \mathrm{H}_{37} \mathrm{FeN}_{3} \mathrm{P}[\mathrm{M}+\mathrm{H}]^{+}: 634.2075$, found: 634.2102.

\section{General Procedure for Pd-Catalyzed Asymmetric [3+2] Cycloaddition of $\beta$-Ketoesters with Propargylic Esters}

A solution of $\mathrm{Pd}_{2}(\mathrm{dba})_{3} \mathrm{CHCl}_{3}(7.8 \mathrm{mg}, 0.0075 \mathrm{mmol})$ and $\left(R_{c}, S_{p}\right)-\mathrm{L} 4 \mathrm{a}(9.2 \mathrm{mg}, 0.0165 \mathrm{mmol})$ in $1 \mathrm{~mL}$ of anhydrous toluene placed in an oven-dried Schlenk flask was stirred at room temperature under a nitrogen atmosphere for $1 \mathrm{~h}$. A solution of $\beta$-ketoesters $1(0.3 \mathrm{mmol})$, propargylic esters $2(0.33 \mathrm{mmol})$ and $\mathrm{Cs}_{2} \mathrm{CO}_{3}(117.3 \mathrm{mg}, 0.36 \mathrm{mmol})$ in $2 \mathrm{~mL}$ of anhydrous toluene was added. The mixture was stirred at room temperature for $20 \mathrm{~h}$. The reaction mixture was purified by silica gel chromatography to afford dihydrofuran products 3 .

(S)-Methyl 4-methylene-2,5-diphenyl-4,5-dihydrofuran-3-carboxylate (3aa). Pale yellow solid

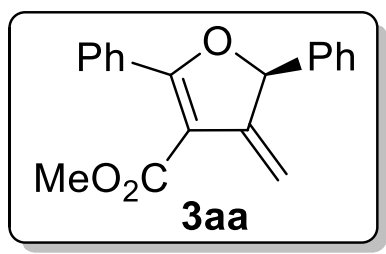

(81 mg, 92\% yield) was obtained after purification with column chromatography on silica gel (hexanes/ethyl acetate, 100/1). M.p.: 63-65 ${ }^{\circ} \mathrm{C} .97 \%$ ee was determined by chiral HPLC (Chiralcel OJ-H, $n$-hexane $/ i-\mathrm{PrOH}=$ 50/50, $0.8 \mathrm{~mL} / \mathrm{min}, 254 \mathrm{~nm}, 40^{\circ} \mathrm{C}$ ): $\mathrm{tR}_{\mathrm{R}}$ (major) $=30.6 \mathrm{~min}, \mathrm{t}_{\mathrm{R}}$ (minor) $=14.8$ min. $[\alpha]_{D^{23}}=-113.1\left(c\right.$ 1.03, $\left.\mathrm{CH}_{2} \mathrm{Cl}_{2}\right) .{ }^{1} \mathrm{H}$ NMR $(400 \mathrm{MHz}$, DMSO-d $): \delta 7.72-7.70(\mathrm{~m}, 2 \mathrm{H})$, 7.55-7.39 (m, 8H), $6.30(\mathrm{t}, J=2.5 \mathrm{~Hz}, 1 \mathrm{H}), 5.50(\mathrm{~d}, J=3.2 \mathrm{~Hz}, 1 \mathrm{H}), 4.67(\mathrm{~d}, J=2.7 \mathrm{~Hz}, 1 \mathrm{H}), 3.66$ 
(s, 3H); ${ }^{13} \mathrm{C}$ NMR (101 MHz, DMSO-d $\left.{ }^{6}\right): \delta 170.5,164.4,148.7,140.3,131.5,130.1,129.4,129.3$, 129.2, 128.5, 127.2, 106.5, 102.9, 87.1, 51.5. HRMS calc. for $\mathrm{C}_{19} \mathrm{H}_{16} \mathrm{O}_{3} \mathrm{Na}[\mathrm{M}+\mathrm{Na}]^{+}: 315.0997$, found: 315.0999 .

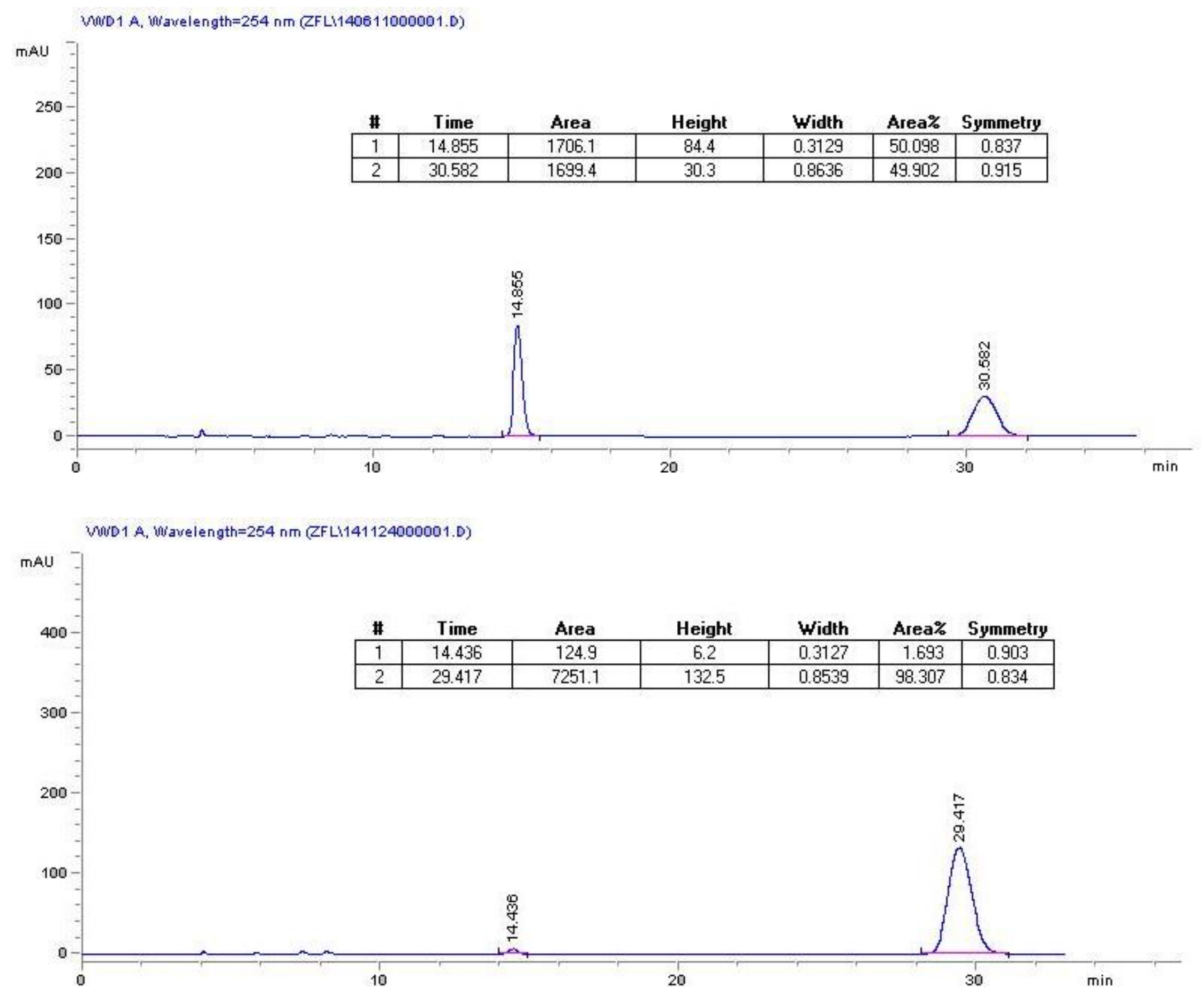

(S)-Methyl 4-methylene-5-phenyl-2-(4-methylphenyl)-4,5-dihydrofuran-3-carboxylate (3ba).

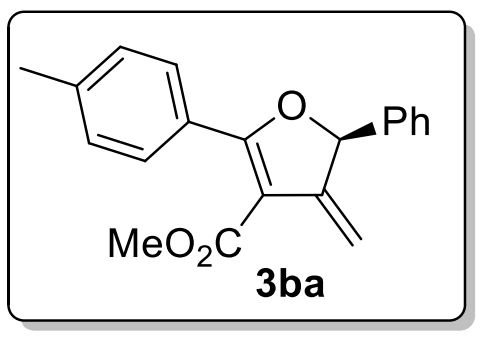

Yellow oil (81 mg, 88\% yield) was obtained after purification with column chromatography on silica gel (hexanes/ethyl acetate, 100/1). $98 \%$ ee was determined by chiral HPLC (Chiralpak AD-H, $n$-hexane/i-PrOH = 90/10, $\left.0.8 \mathrm{~mL} / \mathrm{min}, 254 \mathrm{~nm}, 40^{\circ} \mathrm{C}\right): \mathrm{t}_{\mathrm{R}}$ (major) = $18.8 \mathrm{~min}, \mathrm{t}_{\mathrm{R}}$ (minor) $=9.3 \mathrm{~min} .[\alpha]_{\mathrm{D}}{ }^{23}=-55.9\left(c \mathrm{c} 1.26, \mathrm{CH}_{2} \mathrm{Cl}_{2}\right) .{ }^{1} \mathrm{H}$ NMR (400 MHz, DMSO-d $\left.\mathrm{d}^{6}\right): \delta$ 7.63-7.61 (m, 2H), 7.46-7.37 (m, 5H), 7.28-7.27 (m, 2H), $6.27(\mathrm{t}, \mathrm{J}=$ $2.9 \mathrm{~Hz}, 1 \mathrm{H}), 5.48(\mathrm{~d}, J=3.2 \mathrm{~Hz}, 1 \mathrm{H}), 4.65(\mathrm{~d}, J=2.7 \mathrm{~Hz}, 1 \mathrm{H}), 3.66(\mathrm{~s}, 3 \mathrm{H}), 2.36(\mathrm{~s}, 3 \mathrm{H}) ;{ }^{13} \mathrm{C}$ NMR $\left(101 \mathrm{MHz}, \mathrm{DMSO}-\mathrm{d}^{6}\right): \delta 170.5,164.5,148.8,141.6,140.3,129.4,129.3,129.1,129.0,127.2$, 
127.1, 106.0, 102.5, 86.9, 51.4, 21.5. HRMS calc. for $\mathrm{C}_{20} \mathrm{H}_{18} \mathrm{O}_{3} \mathrm{Na}[\mathrm{M}+\mathrm{Na}]^{+}: 329.1154$, found: 329.1150 .
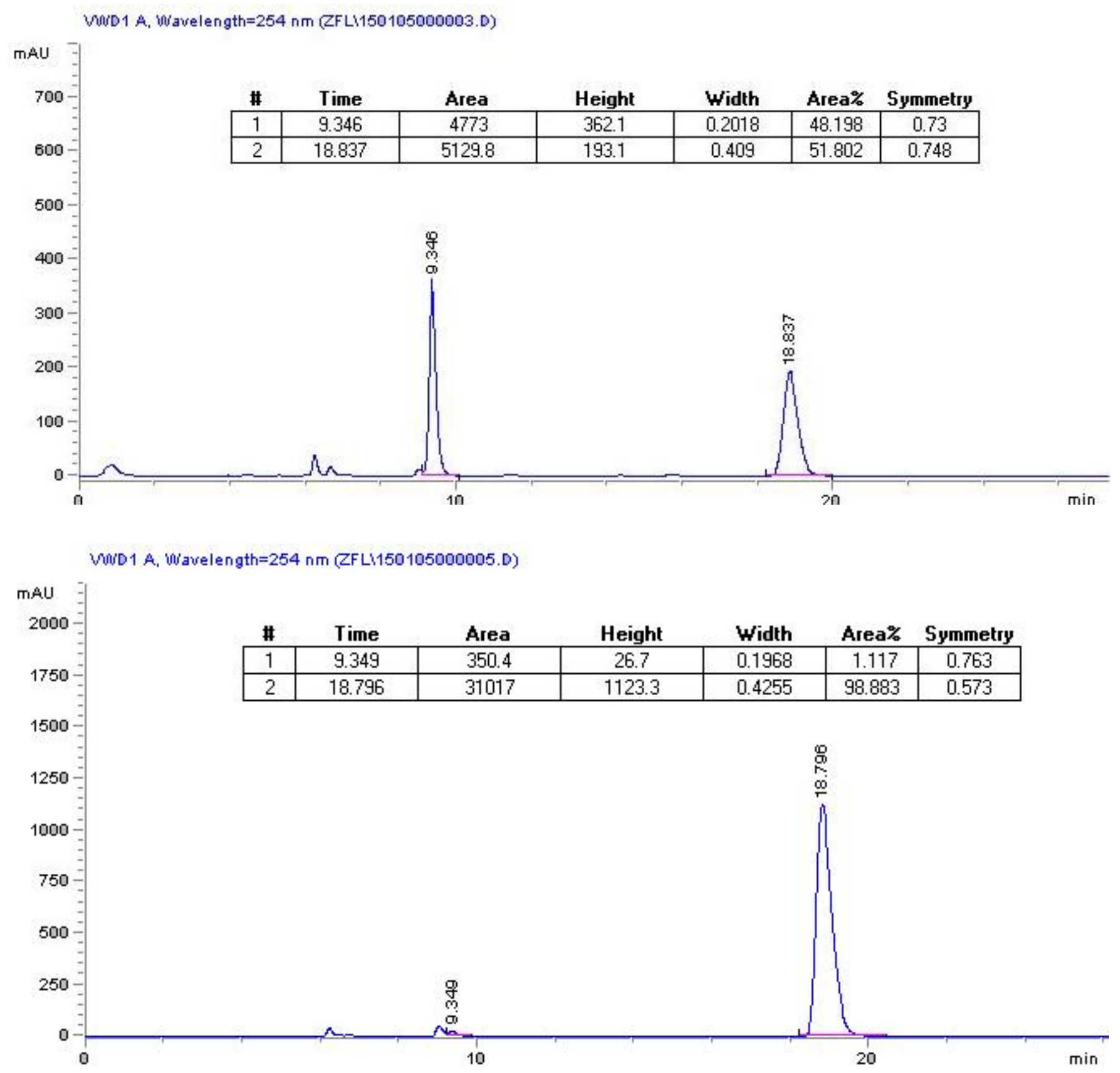

(S)-Methyl 2-(4-methoxyphenyl)-4-methylene-5-phenyl-4,5-dihydrofuran-3-carboxylate

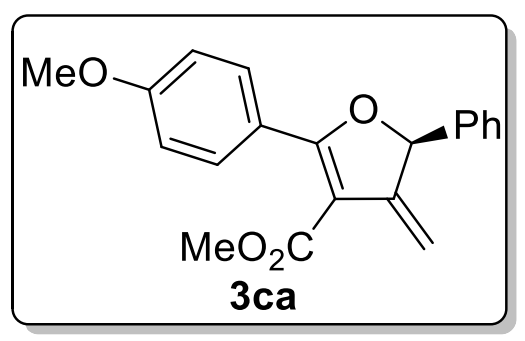

(3ca). Yellow oil ( $77 \mathrm{mg}, 80 \%$ yield) was obtained after purification with column chromatography on silica gel (hexanes/ethyl acetate, 50/1). $98 \%$ ee was determined by chiral HPLC (Chiralpak AD-H, $n$-hexane $/ \mathrm{i}-\mathrm{PrOH}=70 / 30,0.8 \mathrm{~mL} / \mathrm{min}, 254 \mathrm{~nm}, 40^{\circ} \mathrm{C}$ ): $\mathrm{tR}_{\mathrm{R}}$ (major) $=$ $18.3 \mathrm{~min}, \mathrm{t}_{\mathrm{R}}($ minor $)=8.6 \mathrm{~min} .[\alpha]_{\mathrm{D}}{ }^{18}=-23.7\left(c \quad 0.20, \mathrm{CH}_{2} \mathrm{Cl}_{2}\right) .{ }^{1} \mathrm{H}$ 
NMR (400 MHz, DMSO-d $\left.\mathrm{d}^{6}\right): \delta$ 7.73-7.71 (m, 2H), 7.45-7.38 (m, 5H), 7.04-7.01 (m, 2H), $6.25(\mathrm{t}, \mathrm{J}=$ $2.9 \mathrm{~Hz}, 1 \mathrm{H}), 5.45(\mathrm{~d}, J=3.2 \mathrm{~Hz}, 1 \mathrm{H}), 4.62(\mathrm{~d}, J=2.7 \mathrm{~Hz}, 1 \mathrm{H}), 3.82(\mathrm{~s}, 3 \mathrm{H}), 3.68(\mathrm{~s}, 3 \mathrm{H}) ;{ }^{3} \mathrm{C}$ NMR (101 MHz, DMSO-d $\left.{ }^{6}\right): \delta$ 170.3, 164.6, 162.0, 148.9, 140.4, 131.3, 129.3, 129.1, 127.1, 122.1, 113.9, 105.1, 102.1, 86.8, 55.8, 51.4. HRMS calc. for $\mathrm{C}_{20} \mathrm{H}_{18} \mathrm{O}_{4} \mathrm{Na}[\mathrm{M}+\mathrm{Na}]^{+}:$345.1103, found: 345.1101.

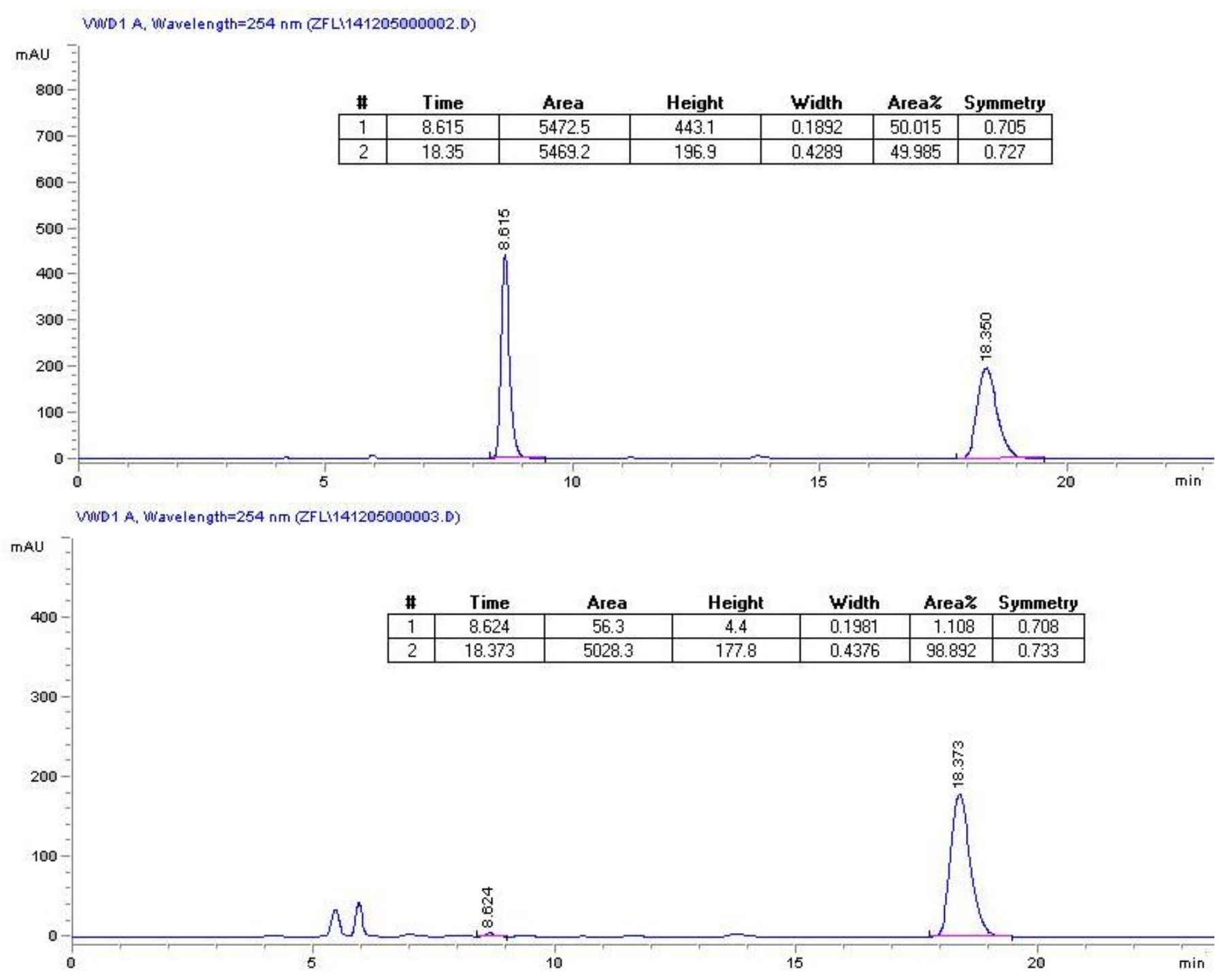

(S)-Methyl 2-(4-fluorophenyl)-4-methylene-5-phenyl-4,5-dihydrofuran-3-carboxylate (3da).

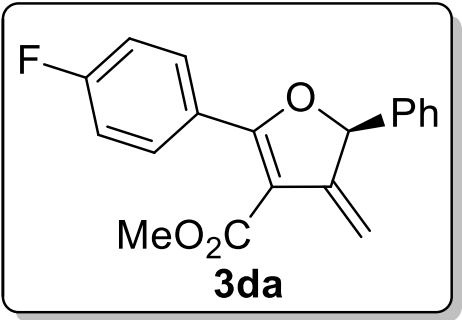

Yellow oil (85 mg, 91\% yield) was obtained after purification with column chromatography on silica gel (hexanes/ethyl acetate, 100/1). 97\% ee was determined by chiral HPLC (Chiralcel OJ-H, $n$-hexane $/ i-\mathrm{PrOH}=50 / 50,0.8 \mathrm{~mL} / \mathrm{min}, 254 \mathrm{~nm}, 40^{\circ} \mathrm{C}$ ): $\mathrm{t}_{\mathrm{R}}$ (major) $=$ $26.6 \mathrm{~min}, \mathrm{t}_{\mathrm{R}}$ (minor) $=9.7 \mathrm{~min} .[\alpha]_{\mathrm{D}}{ }^{19}=-114.8\left(c 0.90, \mathrm{CH}_{2} \mathrm{Cl}_{2}\right) .{ }^{1} \mathrm{H}$ NMR (400 MHz, DMSO-d $)$ : $\delta$ 7.83-7.78 (m, 2H), 7.46-7.29 (m, 7H), $6.30(\mathrm{t}, J=2.9 \mathrm{~Hz}, 1 \mathrm{H}), 5.50$ 
(d, $J=3.2 \mathrm{~Hz}, 1 \mathrm{H}), 4.66(\mathrm{~d}, J=2.7 \mathrm{~Hz}, 1 \mathrm{H}), 3.66(\mathrm{~s}, 3 \mathrm{H}) ;{ }^{13} \mathrm{C}$ NMR (101 MHz, DMSO-d $): \delta 169.6$, 164.3, 163.9 (d, $J=249.4 \mathrm{~Hz}$ ), 148.5, 140.2, 132.1 (d, $J=8.9 \mathrm{~Hz}), 129.3,129.2,127.2,126.5$ (d, $J=3.2 \mathrm{~Hz}), 115.6(\mathrm{~d}, J=21.9 \mathrm{~Hz}), 106.4,103.1,87.1,51.5 ;{ }^{19} \mathrm{~F}$ NMR $\left(376 \mathrm{MHz}\right.$, DMSO-d $\left.{ }^{6}\right): \delta$ -108.5. HRMS calc. for $\mathrm{C}_{19} \mathrm{H}_{15} \mathrm{FO}_{3} \mathrm{Na}[\mathrm{M}+\mathrm{Na}]^{+}: 333.0903$, found: 333.0908 .

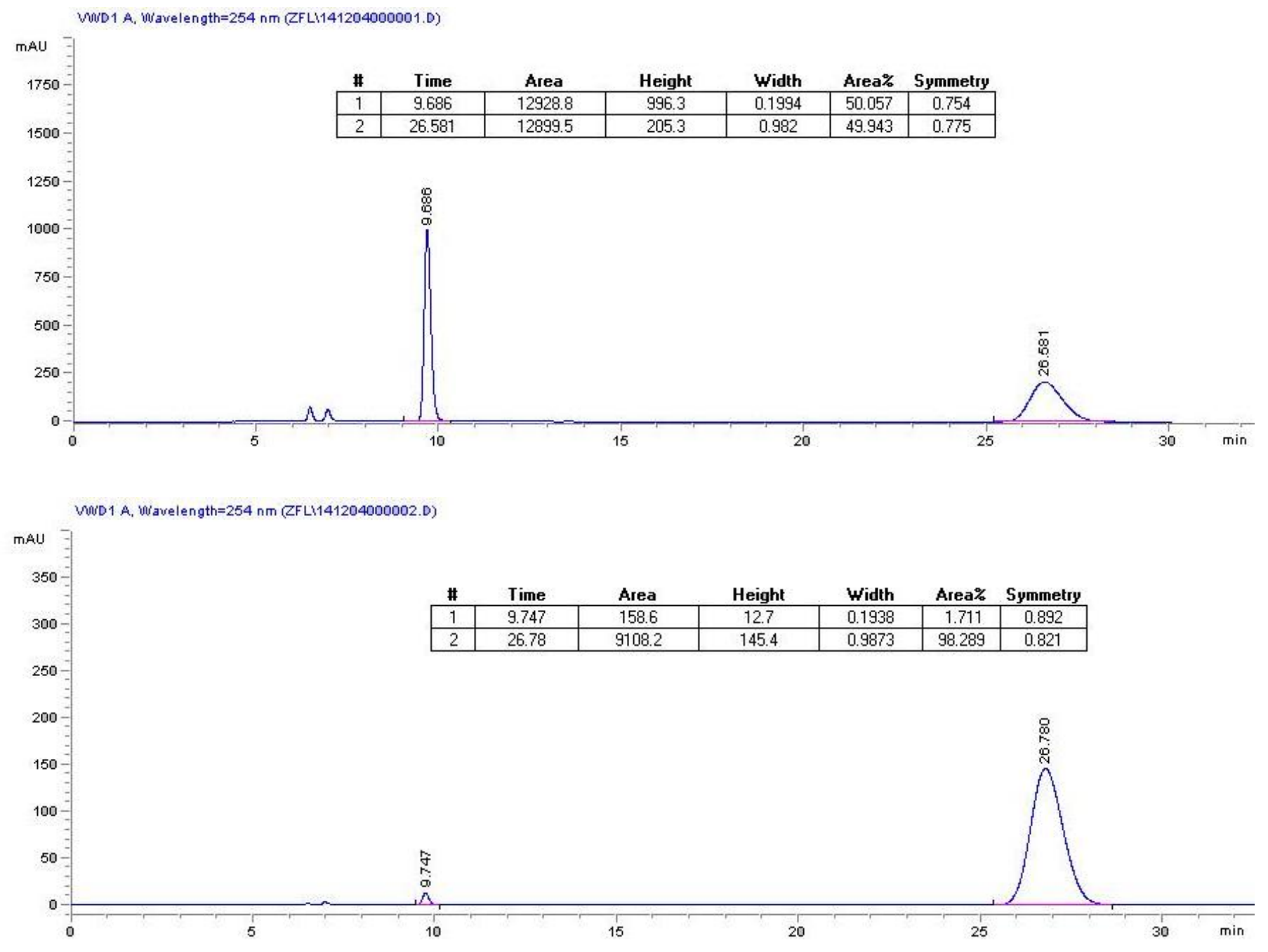

(S)-Methyl 2-(4-bromophenyl)-4-methylene-5-phenyl-4,5-dihydrofuran-3-carboxylate (3ea).

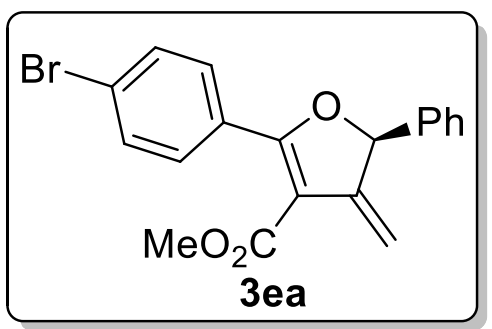

Yellow oil (95 mg, 85\% yield) was obtained after purification with column chromatography on silica gel (hexanes/ethyl acetate, 100/1). 97\% ee was determined by chiral HPLC (Chiralpak AD-H, $n$-hexane $/$ - $\left.\mathrm{PrOH}=80 / 20,0.8 \mathrm{~mL} / \mathrm{min}, 254 \mathrm{~nm}, 40^{\circ} \mathrm{C}\right): \mathrm{tR}_{\mathrm{R}}($ major $)=$ $16.3 \mathrm{~min}, \mathrm{tR}_{\mathrm{R}}($ minor $)=7.8 \mathrm{~min} .[\alpha]_{\mathrm{D}}{ }^{24}=-48.3\left(\mathrm{c} 1.11, \mathrm{CH}_{2} \mathrm{Cl}_{2}\right) .{ }^{1} \mathrm{H}$ NMR (400 MHz, DMSO-d $)$ : $\delta$ 7.70- $7.65(\mathrm{~m}, 4 \mathrm{H}), 7.46-7.39(\mathrm{~m}, 5 \mathrm{H}), 6.30(\mathrm{t}, J=3.0 \mathrm{~Hz}, 1 \mathrm{H}), 5.51$ (d, $J=3.3 \mathrm{~Hz}, 1 \mathrm{H}), 4.68(\mathrm{~d}, J=2.8 \mathrm{~Hz}, 1 \mathrm{H}), 3.67(\mathrm{~s}, 3 \mathrm{H}) ;{ }^{13} \mathrm{C}$ NMR $(101 \mathrm{MHz}$, DMSO-d $): \delta 169.3$, 164.2, 148.5, 140.1, 131.6, 131.4, 129.3, 129.2, 129.2, 127.2, 125.1, 107.0, 103.4, 87.2, 51.6. 
HRMS calc. for $\mathrm{C}_{19} \mathrm{H}_{15} \mathrm{BrO}_{3} \mathrm{Na}[\mathrm{M}+\mathrm{Na}]^{+}:$393.0102, found: 333.0099 .

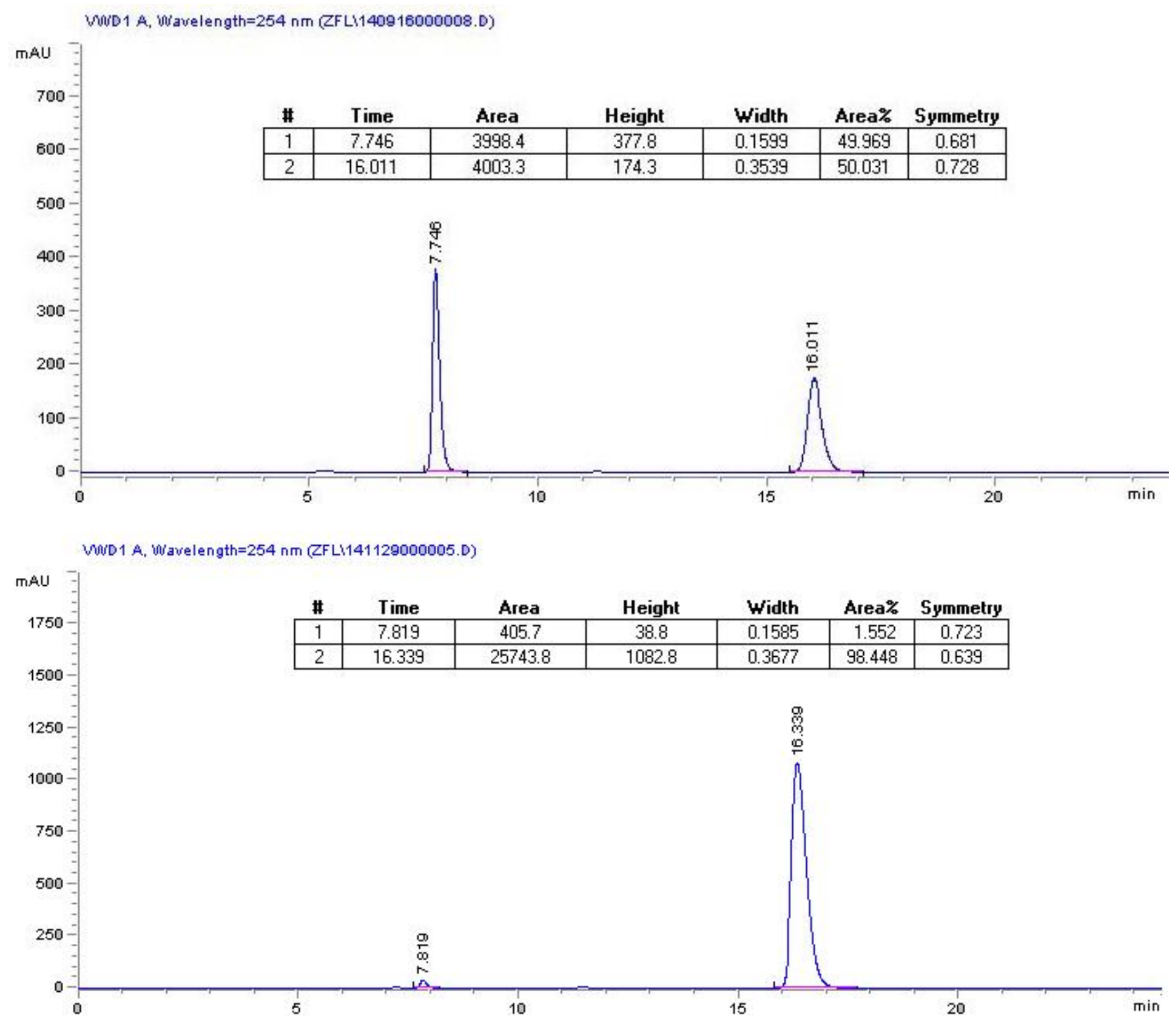

(S)-Methyl 2-(4-chlorophenyl)-4-methylene-5-phenyl-4,5-dihydrofuran-3-carboxylate (3fa).

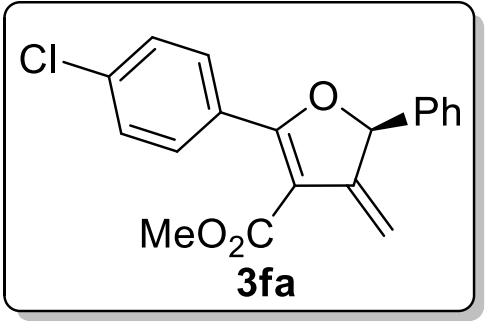

Yellow oil (85 mg, $87 \%$ yield) was obtained after purification with column chromatography on silica gel (hexanes/ethyl acetate, 100/1). 97\% ee was determined by chiral HPLC (Chiralcel OJ-H, $n$-hexane $/ \mathrm{i}-\mathrm{PrOH}=50 / 50,0.8 \mathrm{~mL} / \mathrm{min}, 254 \mathrm{~nm}, 40^{\circ} \mathrm{C}$ ): $\mathrm{t}_{\mathrm{R}}$ (major) $=$ $17.9 \mathrm{~min}, \mathrm{t}_{\mathrm{R}}$ (minor) $=8.0 \mathrm{~min} .[\alpha]_{\mathrm{D}}{ }^{25}=-58.9\left(\mathrm{c} 1.04, \mathrm{CH}_{2} \mathrm{Cl}_{2}\right) .{ }^{1} \mathrm{H}$ NMR (400 MHz, DMSO-d $): \delta$ 7.75-7.72 (m, 2H), 7.56-7.50 (m, 2H), 7.45- 7.35 (m, 5H), $6.29(\mathrm{t}, J$ $=3.0 \mathrm{~Hz}, 1 \mathrm{H}), 5.52(\mathrm{~d}, J=3.3 \mathrm{~Hz}, 1 \mathrm{H}), 4.68(\mathrm{~d}, J=2.7 \mathrm{~Hz}, 1 \mathrm{H}), 3.66(\mathrm{~s}, 3 \mathrm{H}) ;{ }^{13} \mathrm{C} \mathrm{NMR}(101 \mathrm{MHz}$, DMSO-d $): \delta$ 169.2, 164.2, 148.4, 140.1, 136.2, 131.3, 129.3, 129.2, 128.9, 128.6, 127.2, 107.0, 
103.4, 87.3, 51.5. HRMS calc. for $\mathrm{C}_{19} \mathrm{H}_{15} \mathrm{ClO}_{3} \mathrm{Na}[\mathrm{M}+\mathrm{Na}]^{+}: 349.0607$, found: 349.0608 .

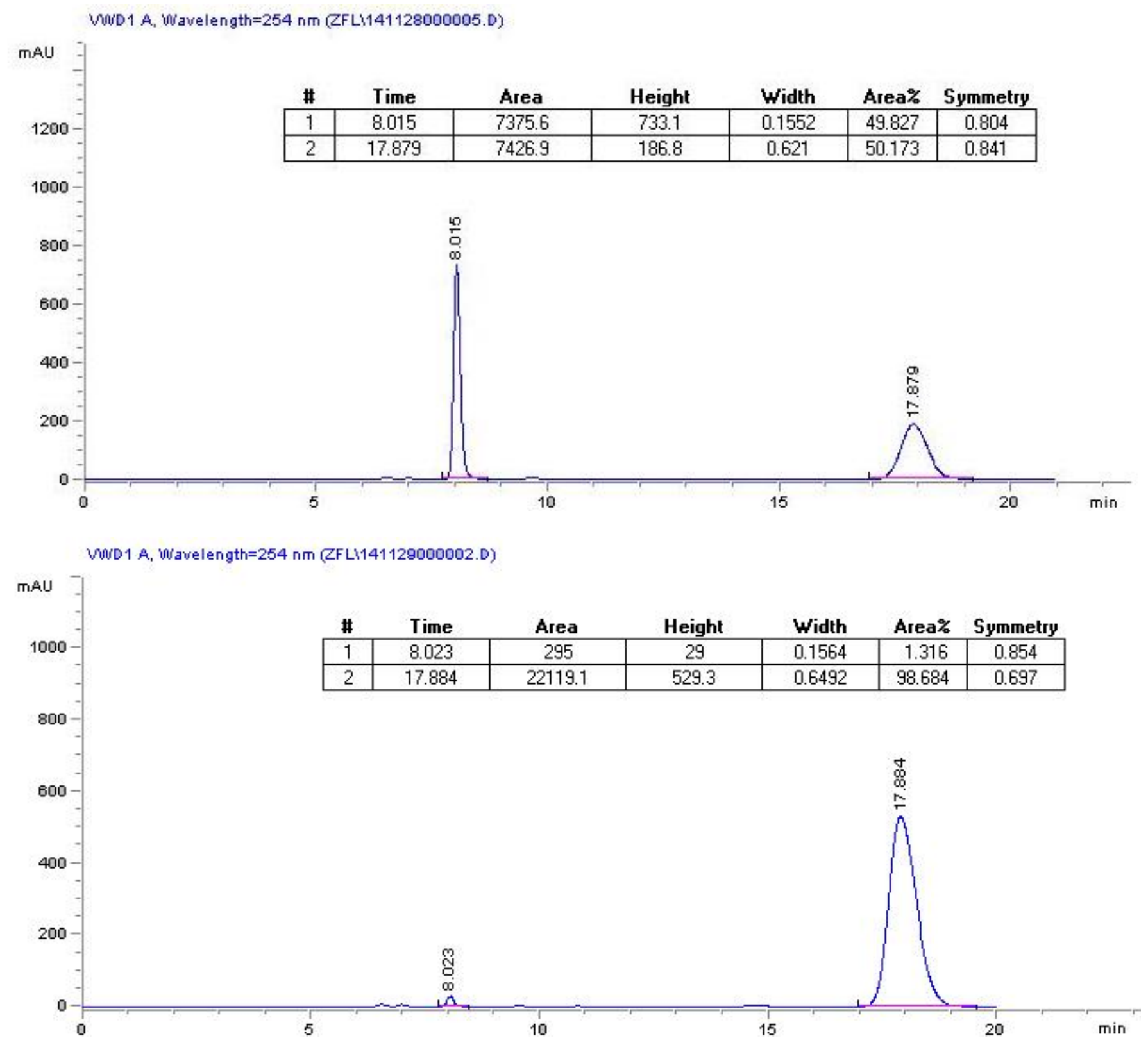

(S)-Methyl 2-(3-chlorophenyl)-4-methylene-5-phenyl-4,5-dihydrofuran-3-carboxylate (3ga).

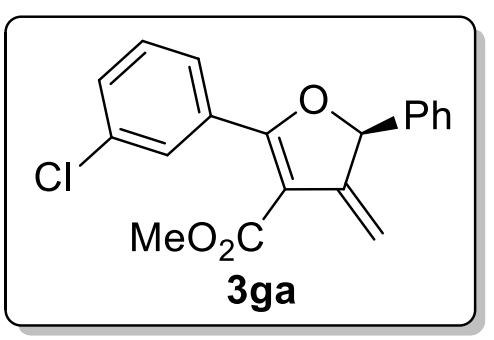

Yellow oil (91 $\mathrm{mg}, 93 \%$ yield) was obtained after purification with column chromatography on silica gel (hexanes/ethyl acetate, 100/1). $96 \%$ ee was determined by chiral HPLC (Chiralcel OJ-H, $n$-hexane $/$ i-PrOH $\left.=80 / 20,0.8 \mathrm{~mL} / \mathrm{min}, 254 \mathrm{~nm}, 40^{\circ} \mathrm{C}\right): \mathrm{tR}_{\mathrm{R}}($ major $)=$ $18.2 \mathrm{~min}, \mathrm{t}_{\mathrm{R}}$ (minor) $=10.6 \mathrm{~min} .[\alpha]_{\mathrm{D}^{25}}=-69.6\left(\mathrm{c} \mathrm{1.11}, \mathrm{CH}_{2} \mathrm{Cl}_{2}\right) .{ }^{1} \mathrm{H}$ NMR (400 MHz, DMSO-d 6 ): $\delta$ 7.78-7.77 (m, 1H), 7.69-7.67 (m, 1H), 7.60-7.37 (m, 7H), $6.30(\mathrm{t}, J=$ $2.9 \mathrm{~Hz}, 1 \mathrm{H}), 5.55(\mathrm{~d}, J=3.2 \mathrm{~Hz}, 1 \mathrm{H}), 4.69(\mathrm{~d}, J=2.7 \mathrm{~Hz}, 1 \mathrm{H}), 3.67(\mathrm{~s}, 3 \mathrm{H}) ;{ }^{13} \mathrm{C} \mathrm{NMR}(101 \mathrm{MHz}$, DMSO-d $): \delta 168.8,164.1,148.3,140.0,133.2,132.1,131.3,130.4,129.3,129.2,129.1,128.1$, 
127.3, 107.4, 103.7, 87.4, 51.6. HRMS calc. for $\mathrm{C}_{19} \mathrm{H}_{15} \mathrm{ClO}_{3} \mathrm{Na}[\mathrm{M}+\mathrm{Na}]^{+}: 349.0607$, found: 349.0598 .

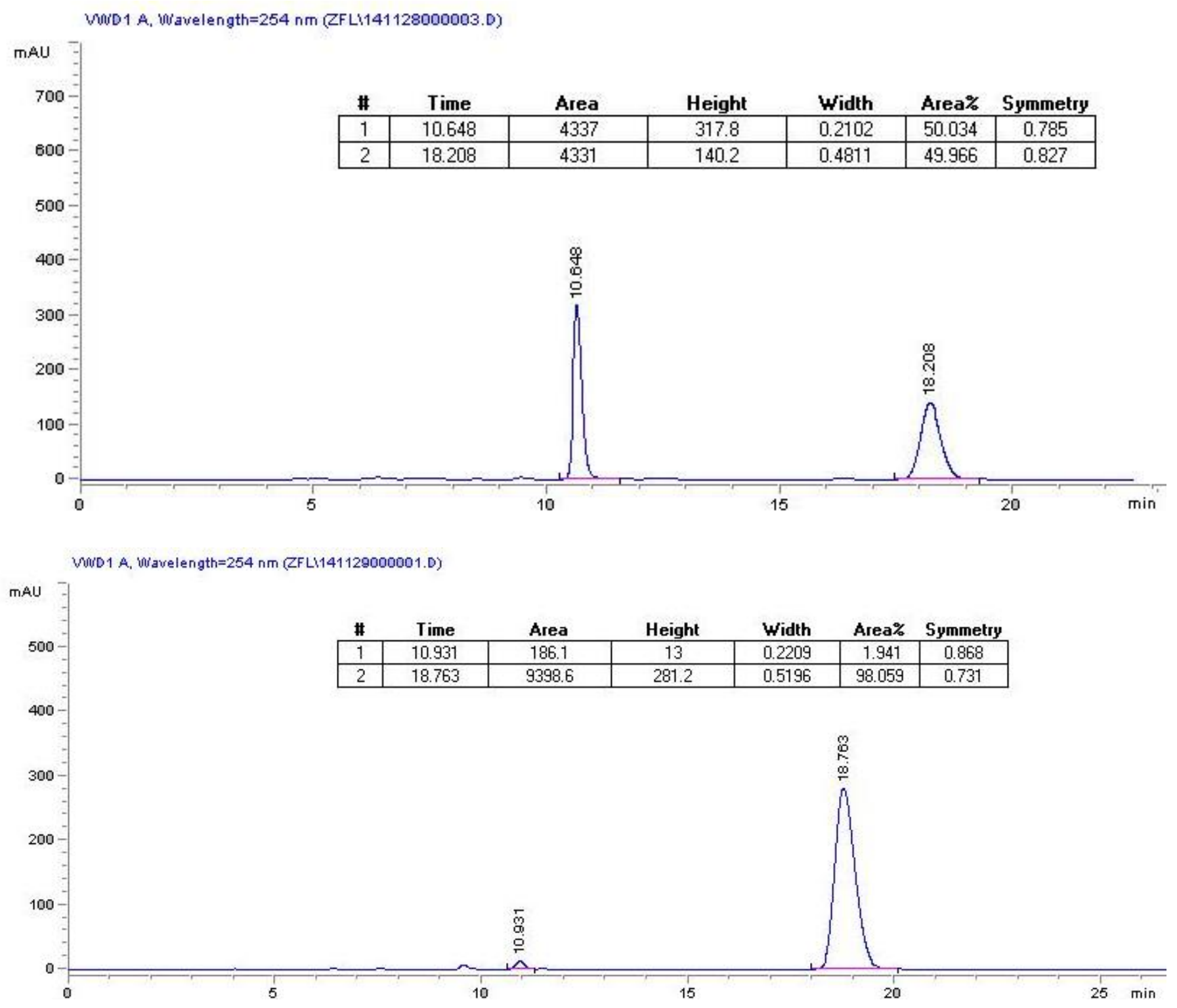

(S)-Methyl 2-(2-chlorophenyl)-4-methylene-5-phenyl-4,5-dihydrofuran-3-carboxylate (3ha).

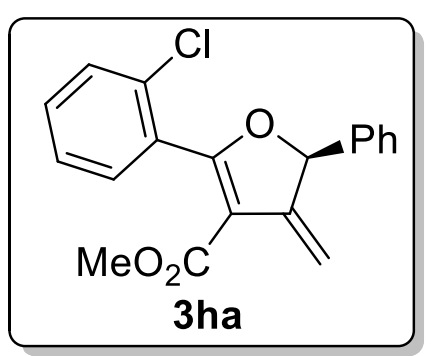

Yellow oil ( $71 \mathrm{mg}, 72 \%$ yield) was obtained after purification with column chromatography on silica gel (hexanes/ethyl acetate, 50/1). 95\% ee was determined by chiral HPLC (Chiralpak AD-H, $n$-hexane/ $\mathrm{-PrOH}=95 / 5$, $\left.0.8 \mathrm{~mL} / \mathrm{min}, 254 \mathrm{~nm}, 40^{\circ} \mathrm{C}\right)$ : $\mathrm{t}_{\mathrm{R}}($ major $)=11.3 \mathrm{~min}, \mathrm{t}_{\mathrm{R}}($ minor $)=10.1 \mathrm{~min}$. $[\alpha]_{D}{ }^{16}=-73.1$ (c 0.20, $\left.\mathrm{CH}_{2} \mathrm{Cl}_{2}\right) .{ }^{1} \mathrm{H}$ NMR $\left(400 \mathrm{MHz}, \mathrm{DMSO}-\mathrm{d}^{6}\right): \delta$ 7.60-7.40 (m, 9H), $6.37(\mathrm{t}, J=3.0 \mathrm{~Hz}, 1 \mathrm{H}), 5.60(\mathrm{~d}, J=3.3 \mathrm{~Hz}, 1 \mathrm{H}), 4.67$ (d, $J=2.8 \mathrm{~Hz}, 1 \mathrm{H}), 3.53(\mathrm{~s}, 3 \mathrm{H}) ;{ }^{13} \mathrm{C}$ NMR $(101 \mathrm{MHz}$, DMSO-d $): \delta 169.5,163.5,147.6,139.9$, 
132.4, 132.2, 131.2, 130.7, 129.9, 129.3, 129.3, 127.5, 127.5, 109.2, 103.4, 88.4, 51.5. HRMS calc. for $\mathrm{C}_{19} \mathrm{H}_{15} \mathrm{ClO}_{3} \mathrm{Na}[\mathrm{M}+\mathrm{Na}]^{+}:$349.0607, found: 349.0599 .

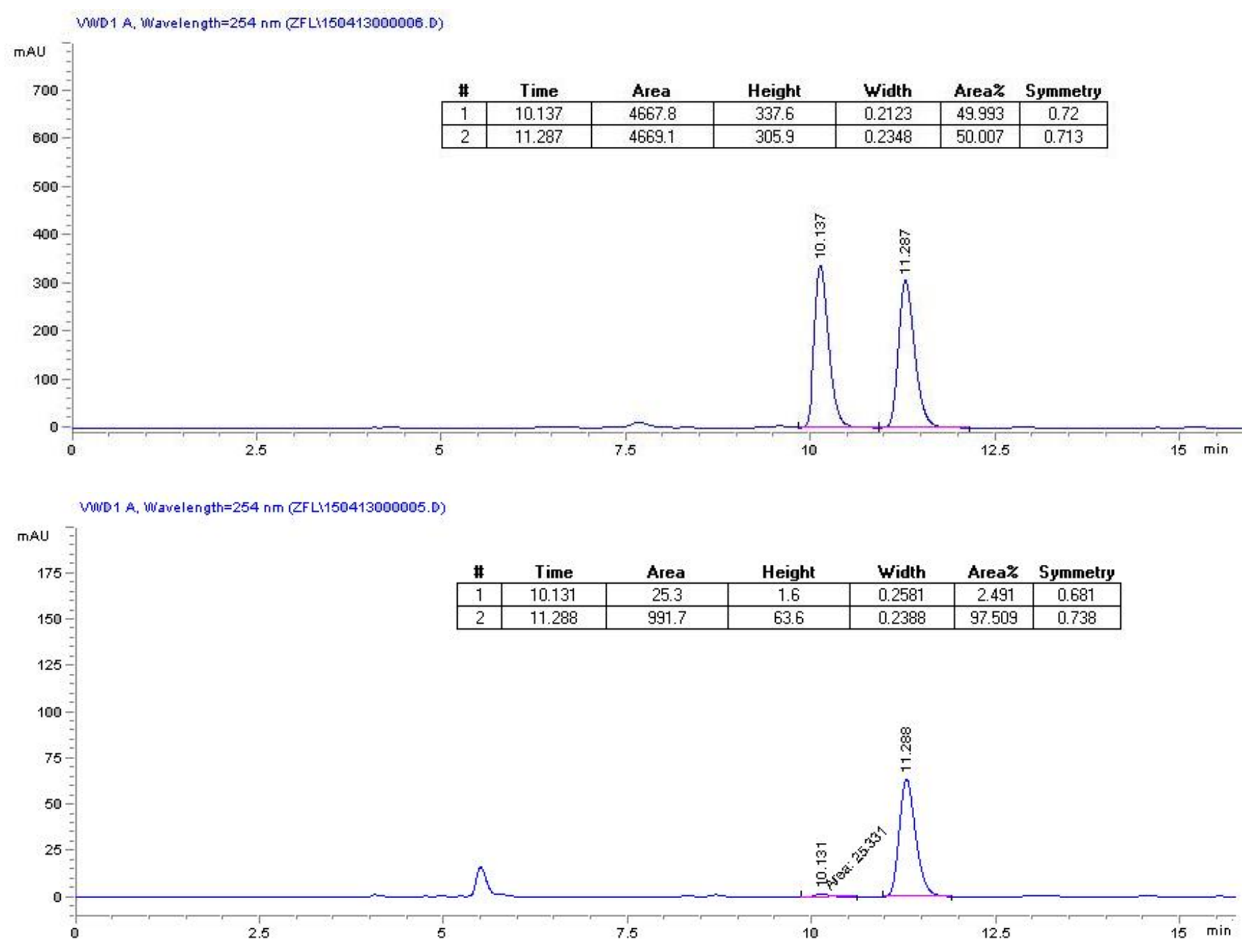

(S)-Methyl 4-methylene-2-(naphthalen-2-yl)-5-phenyl-4,5-dihydrofuran-3-carboxylate (3ia).

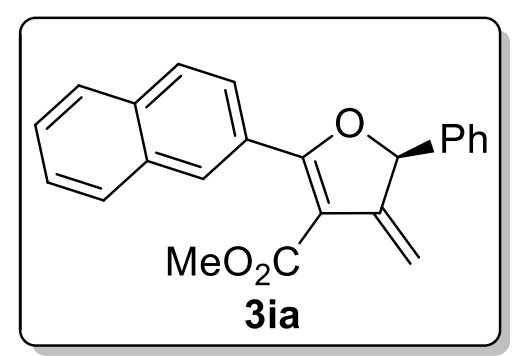

Yellow oil (93 mg, 91\% yield) was obtained after purification with column chromatography on silica gel (hexanes/ethyl acetate, 50/1). $96 \%$ ee was determined by chiral HPLC (Chiralpak AD-H, $n$-hexane $/ i-\mathrm{PrOH}=70 / 30,0.8 \mathrm{~mL} / \mathrm{min}, 254 \mathrm{~nm}, 40^{\circ} \mathrm{C}$ ): tR (major) $=$ $10.7 \mathrm{~min}, \mathrm{t}_{\mathrm{R}}$ (minor) $=7.6 \mathrm{~min} .[\alpha]_{\mathrm{D}}{ }^{25}=-31.7\left(\right.$ c $\left.1.00, \mathrm{CH}_{2} \mathrm{Cl}_{2}\right) .{ }^{1} \mathrm{H}$ NMR (400 MHz, DMSO-d $\left.)^{6}\right): \delta 8.35(\mathrm{~s}, 1 \mathrm{H}), 8.05-7.97(\mathrm{~m}, 3 \mathrm{H})$, 7.78-7.75 (m, 1H), 7.64-7.55 (m, 2H), 7.48-7.39 (m, 5H), $6.36(\mathrm{t}, J=3.0 \mathrm{~Hz}, 1 \mathrm{H}), 5.55(\mathrm{~d}, J=3.3$ $\mathrm{Hz}, 1 \mathrm{H}), 4.70(\mathrm{~d}, J=2.7 \mathrm{~Hz}, 1 \mathrm{H}), 3.68(\mathrm{~s}, 3 \mathrm{H}) ;{ }^{13} \mathrm{C}$ NMR (101 MHz, DMSO-d $\left.)^{6}\right): \delta 170.3,164.4$, $148.8,140.3,134.3,132.5,129.7,129.3,129.3,129.2,128.3,128.0,127.8,127.5,127.3,127.2$, 
126.1, 106.9, 103.0, 87.3, 51.5. HRMS calc. for $\mathrm{C}_{23} \mathrm{H}_{18} \mathrm{O}_{3} \mathrm{Na}[\mathrm{M}+\mathrm{Na}]^{+}: 365.1154$, found: 365.1152 .

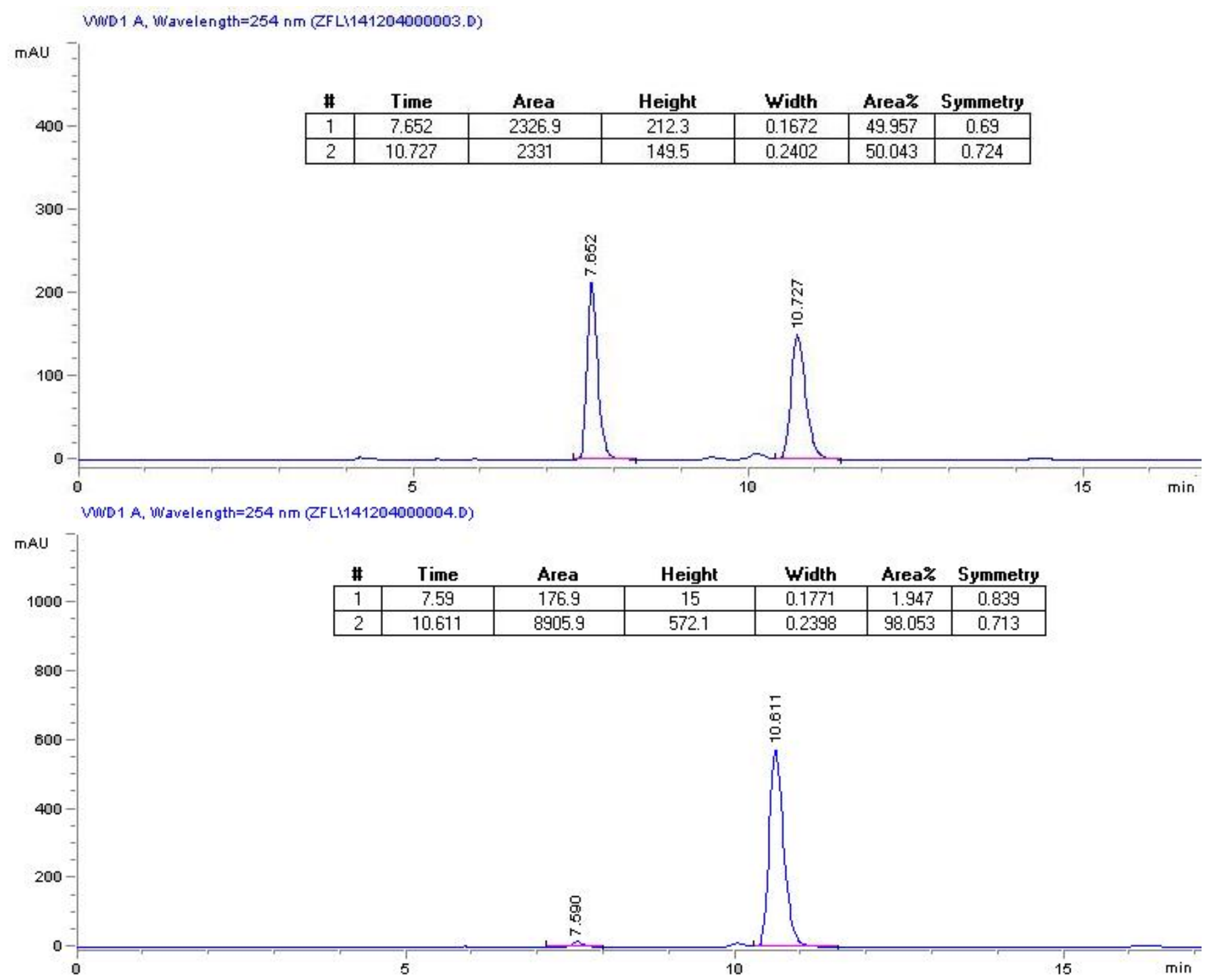

(S)-Methyl 4-methylene-5-phenyl-2-(thiophen-2-yl)-4,5-dihydrofuran-3-carboxylate (3ja).

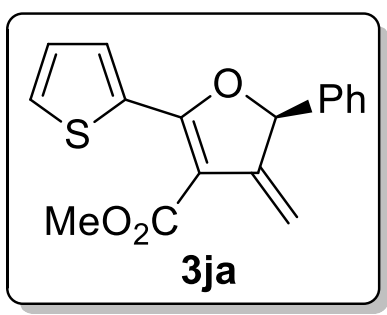

Yellow oil $(79 \mathrm{mg}, 88 \%$ yield) was obtained after purification with column chromatography on silica gel (hexanes/ethyl acetate, 100/1). 97\% ee was determined by chiral HPLC (Chiralpak AD-H, $n$-hexane/ $i-\mathrm{PrOH}=90 / 10$, $\left.0.8 \mathrm{~mL} / \mathrm{min}, 254 \mathrm{~nm}, 40^{\circ} \mathrm{C}\right): \mathrm{t}_{\mathrm{R}}$ (major) $=8.6 \mathrm{~min}, \mathrm{t}_{\mathrm{R}}($ minor $)=7.3 \mathrm{~min}$. $[\alpha]_{D}{ }^{18}=-3.10\left(c 1.01, \mathrm{CH}_{2} \mathrm{Cl}_{2}\right) .{ }^{1} \mathrm{H}$ NMR $\left(400 \mathrm{MHz}, \mathrm{DMSO}-\mathrm{d}^{6}\right): \delta 8.15$ (dd, $J=3.8,1.0 \mathrm{~Hz}, 1 \mathrm{H}), 7.96(\mathrm{dd}, J=5.0,1.0 \mathrm{~Hz}, 1 \mathrm{H}), 7.44-7.37(\mathrm{~m}, 5 \mathrm{H}), 7.24(\mathrm{dd}, J=4.9,4.0 \mathrm{~Hz}$, $1 \mathrm{H}), 6.28(\mathrm{t}, J=2.8 \mathrm{~Hz}, 1 \mathrm{H}), 5.52(\mathrm{~d}, J=3.1 \mathrm{~Hz}, 1 \mathrm{H}), 4.67(\mathrm{~d}, J=2.6 \mathrm{~Hz}, 1 \mathrm{H}), 3.81(\mathrm{~s}, 3 \mathrm{H}) ;{ }^{13} \mathrm{C}$ NMR (101 MHz, DMSO-d 6 ): $\delta 164.5,163.9,148.2,140.4,133.9,133.5,130.8,129.3,129.2$, 128.3, 127.2, 104.4, 103.6, 86.7, 51.6. HRMS calc. for $\mathrm{C}_{17} \mathrm{H}_{14} \mathrm{O}_{3} \mathrm{SNa}[\mathrm{M}+\mathrm{Na}]^{+}: 321.0561$, found: 321.0560 . 


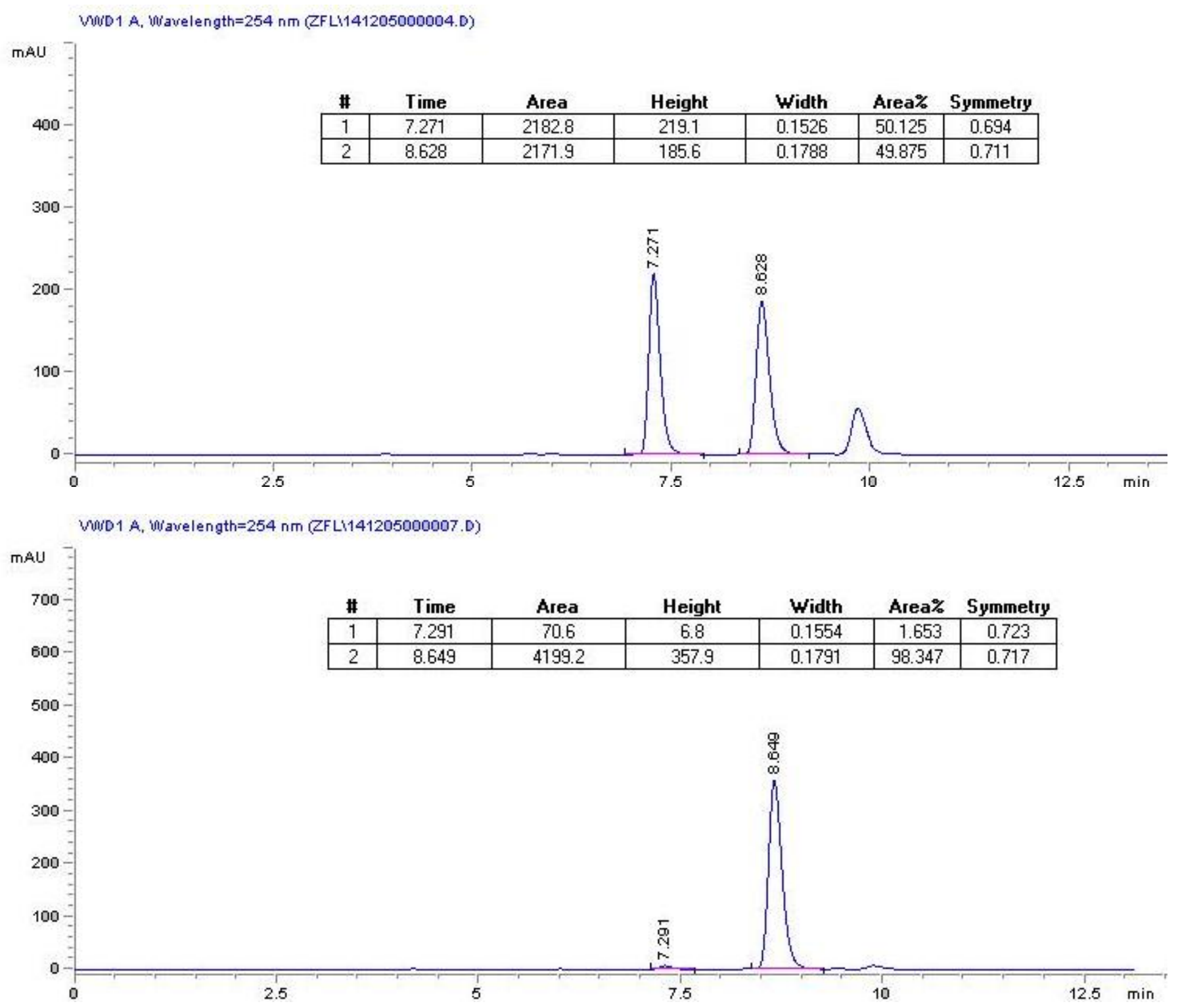

(S)-Methyl 2-methyl-4-methylene-5-phenyl-4,5-dihydrofuran-3-carboxylate (3ka). Colorless

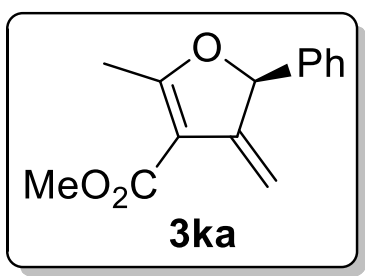
oil (52 mg, 76\% yield) was obtained after purification with column chromatography on silica gel (hexanes/ethyl acetate, 70/1). $91 \%$ ee was determined by chiral HPLC (Chiralcel OJ-H, $n$-hexane/i-PrOH $=50 / 50,0.8$ $\left.\mathrm{mL} / \mathrm{min}, 254 \mathrm{~nm}, 40^{\circ} \mathrm{C}\right): \mathrm{t}_{\mathrm{R}}($ major$)=19.1 \mathrm{~min}, \mathrm{t}_{\mathrm{R}}($ minor $)=11.4 \mathrm{~min} .[\alpha]_{\mathrm{D}}{ }^{18}$ $=-172.5\left(c 0.50, \mathrm{CH}_{2} \mathrm{Cl}_{2}\right) .{ }^{1} \mathrm{H}$ NMR $\left(400 \mathrm{MHz}, \mathrm{DMSO}-\mathrm{d}^{6}\right): \delta$ 7.43-7.33 $(\mathrm{m}$, $5 \mathrm{H}$ ), 6.14 (t, $J=2.9 \mathrm{~Hz}, 1 \mathrm{H}$ ), $5.39(\mathrm{~d}, J=3.1 \mathrm{~Hz}, 1 \mathrm{H}), 4.48(\mathrm{~d}, J=2.6 \mathrm{~Hz}, 1 \mathrm{H}), 3.73(\mathrm{~s}, 3 \mathrm{H}), 2.39$ (s, $3 \mathrm{H}) ;{ }^{13} \mathrm{C}$ NMR (101 MHz, DMSO-d $): \delta$ 176.2, 164.8, 147.6, 140.0, 129.2, 129.1, 127.3, 106.0, 100.8, 87.7, 51.3, 15.8. HRMS calc. for $\mathrm{C}_{14} \mathrm{H}_{14} \mathrm{O}_{3} \mathrm{Na}[\mathrm{M}+\mathrm{Na}]^{+}: 253.0841$, found: 253.0849 . 
MAU

\begin{tabular}{rl|c|c|c|c|c|c|}
\multicolumn{1}{c}{ \# } & \multicolumn{1}{c}{ Time } & \multicolumn{1}{c}{ Area } & Height & \multicolumn{2}{c|}{ Width Area\% Symmetry } \\
\hline 1 & 11.455 & 2489.1 & 191.3 & 0.2027 & 49.968 & 0.936 \\
\hline 2 & 19.082 & 2492.3 & 106.8 & 0.3655 & 50.032 & 0.972 \\
\hline
\end{tabular}

400

300

300

20

100



$0-$

mAL

500

500

400

300

200

100

100

0

Woid 1 A, Wiavelength $=254 \mathrm{~nm}$ (ZFL:150207000004.D)

\begin{tabular}{|c|c|c|c|c|c|c|}
\hline$\#$ & Time & Area & Height & width & Area\% & Symmetry \\
\hline 1 & 11.3 & 263.7 & 20 & 0.2044 & 4.299 & 0.946 \\
\hline 2 & 18.894 & 5868.8 & 247.7 & 0.3696 & 95.701 & 1.021 \\
\hline
\end{tabular}

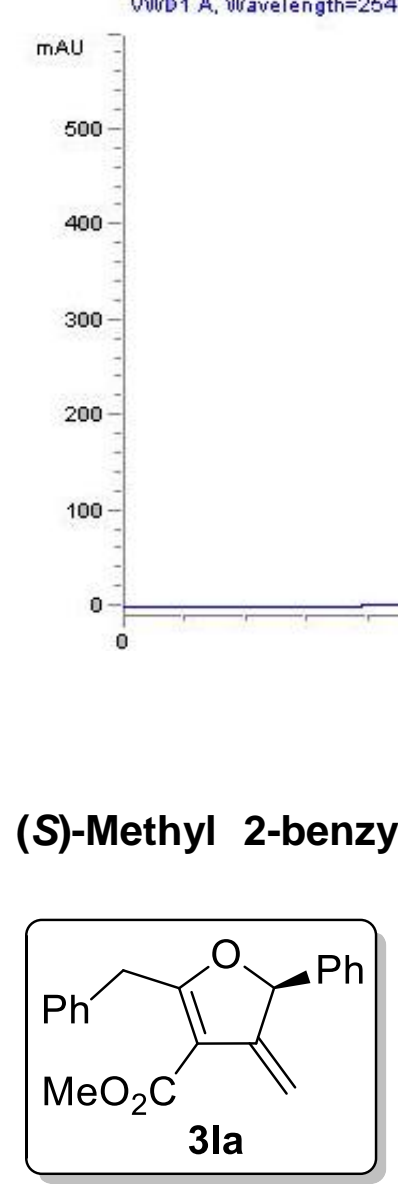

(S)-Methyl 2-benzyl-4-methylene-5-phenyl-4,5-dihydrofuran-3-carboxylate (3la). Colorless oil (64 mg, 70\% yield) was obtained after purification with column

chromatography on silica gel (hexanes/ethyl acetate, 200/1). 96\% ee was determined by chiral HPLC (Chiralpak AD-H, $n$-hexane/ $i$-PrOH $=90 / 10,0.8$ $\left.\mathrm{mL} / \mathrm{min}, 254 \mathrm{~nm}, 40^{\circ} \mathrm{C}\right): \mathrm{t}_{\mathrm{R}}($ major $)=9.3 \mathrm{~min}, \mathrm{t}_{\mathrm{R}}($ minor $)=6.0 \mathrm{~min} .[\alpha]_{\mathrm{D}^{25}}=$ -104.1 (c 0.83, $\left.\mathrm{CH}_{2} \mathrm{Cl}_{2}\right)$. ${ }^{1} \mathrm{H}$ NMR (400 MHz, DMSO-d $\left.{ }^{6}\right): \delta$ 7.35-7.20 (m, $10 \mathrm{H}), 6.14$ (t, J=2.9 Hz, 1H), 5.45 (d, J=3.0 Hz, 1H), 4.53 (d, J=2.6 Hz, 1H), 4.18 (s, $2 \mathrm{H}), 3.78$ (s, 3H); ${ }^{13} \mathrm{C}$ NMR (101 MHz, DMSO-d 6 ): $\delta$ 176.8, 164.7, 147.5, 140.1, 136.5, 129.3, 129.1, 129.1, 129.0, 127.3, 127.1, 106.1, 101.9, 87.6, 51.5, 34.7. HRMS calc. for $\mathrm{C}_{20} \mathrm{H}_{18} \mathrm{O}_{3} \mathrm{Na}[\mathrm{M}+\mathrm{Na}]^{+}$: 329.1154, found: 329.1152. 

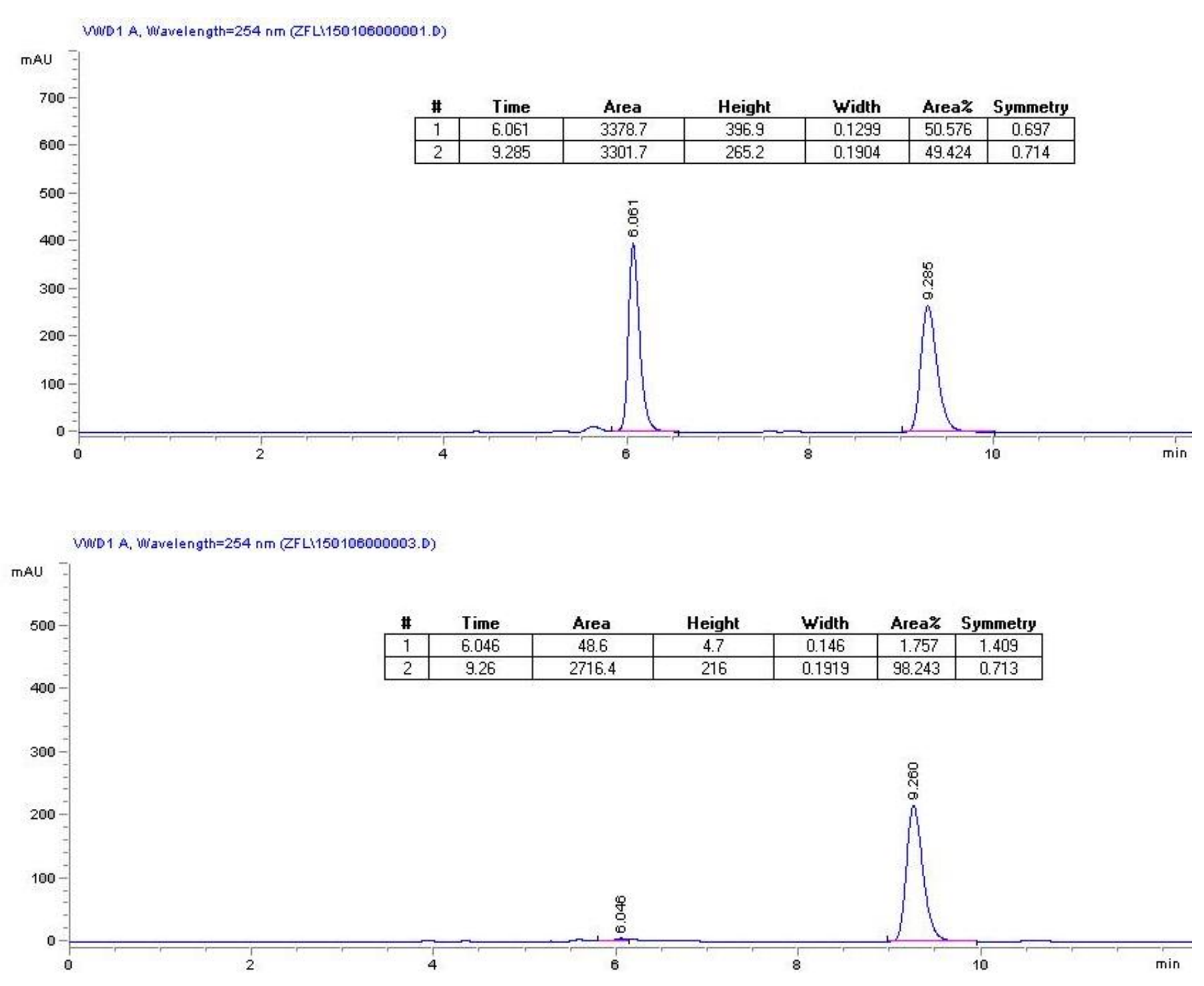

(S)-Methyl 4-methylene-2-phenyl-5-(4-methylphenyl)-4,5-dihydrofuran-3-carboxylate (3ab).

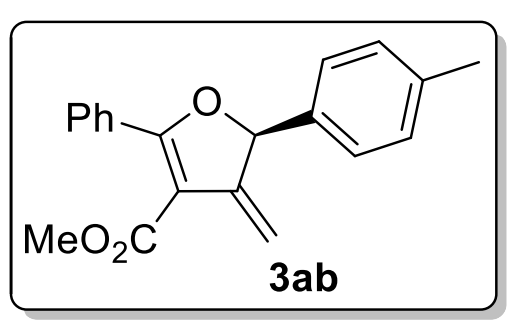

Yellow oil (86 $\mathrm{mg}, 94 \%$ yield) was obtained after purification with column chromatography on silica gel (hexanes/ethyl acetate, 100/1). 97\% ee was determined by chiral HPLC (Chiralpak AD-H, $n$-hexane $\left./ \mathrm{i}-\mathrm{PrOH}=90 / 10,0.8 \mathrm{~mL} / \mathrm{min}, 254 \mathrm{~nm}, 40^{\circ} \mathrm{C}\right): \mathrm{t}_{\mathrm{R}}($ major $)=$ $12.4 \mathrm{~min}, \mathrm{t}_{\mathrm{R}}$ (minor) $=7.7 \mathrm{~min} .[\alpha]_{\mathrm{D}}{ }^{25}=-80.8\left(\mathrm{c} 1.15, \mathrm{CH}_{2} \mathrm{Cl}_{2}\right) .{ }^{1} \mathrm{H}$ NMR (400 MHz, DMSO-d $): \delta$ 7.71-7.68 (m, 2H), 7.54-7.44 (m, 3H), 7.31-7.22 (m, 4H), $6.25(\mathrm{t}, J=$ $2.9 \mathrm{~Hz}, 1 \mathrm{H}), 5.50(\mathrm{~d}, J=3.3 \mathrm{~Hz}, 1 \mathrm{H}), 4.63(\mathrm{~d}, J=2.7 \mathrm{~Hz}, 1 \mathrm{H}), 3.65(\mathrm{~s}, 1 \mathrm{H}), 2.31(\mathrm{~s}, 3 \mathrm{H}) ;{ }^{13} \mathrm{C} \mathrm{NMR}$ (101 MHz, DMSO-d6): $\delta 170.5,164.4,148.8,138.6,137.3,131.5,130.2,129.8,129.4,128.4$, 127.3, 106.5, 102.7, 87.2, 51.4, 21.2. HRMS calc. for $\mathrm{C}_{20} \mathrm{H}_{19} \mathrm{O}_{3}[\mathrm{M}+\mathrm{H}]^{+}: 307.1334$, found: 307.1335 . 


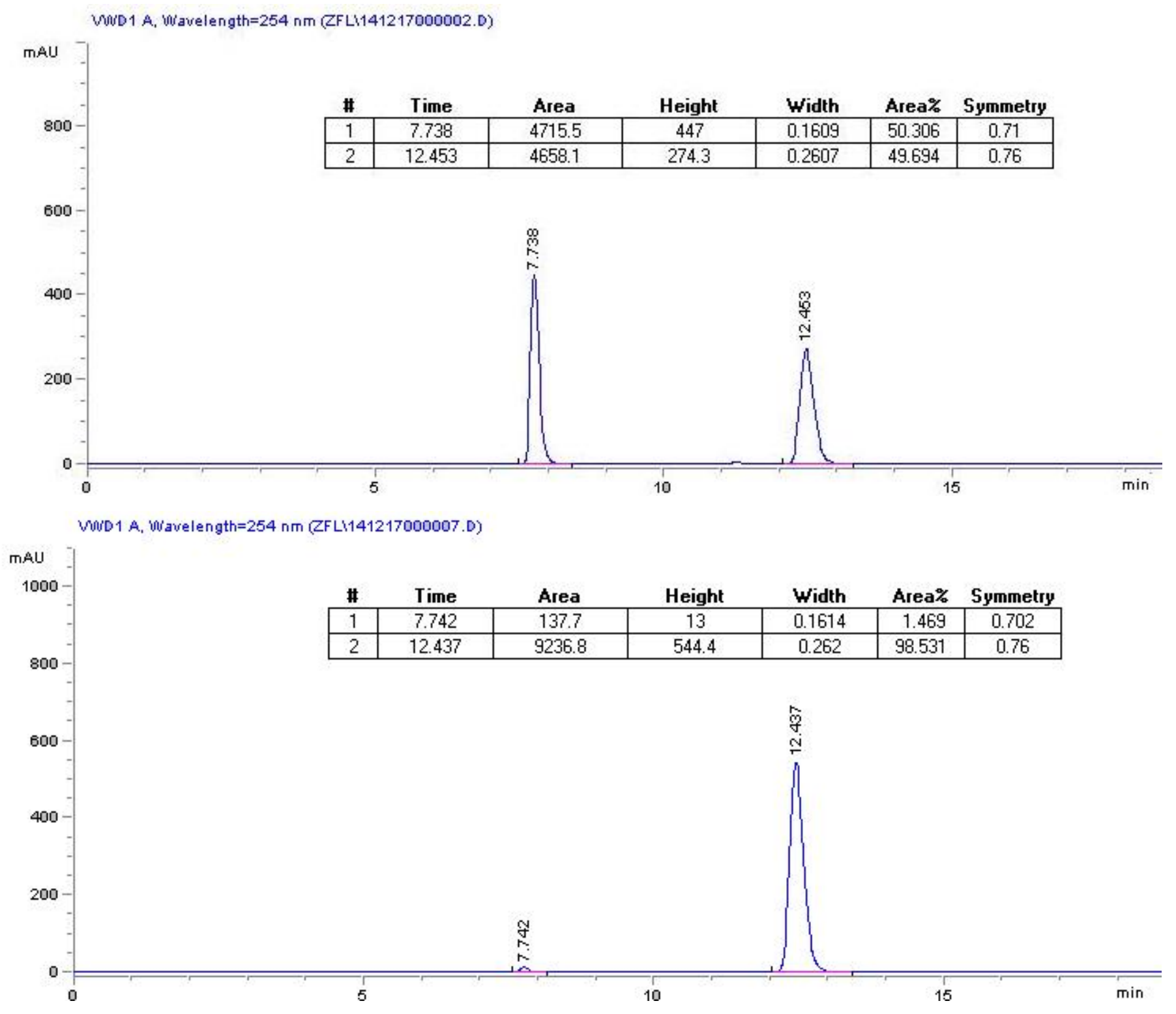

(S)-Methyl 5-(4-methoxyphenyl)-4-methylene-2-phenyl-4,5-dihydrofuran-3-carboxylate

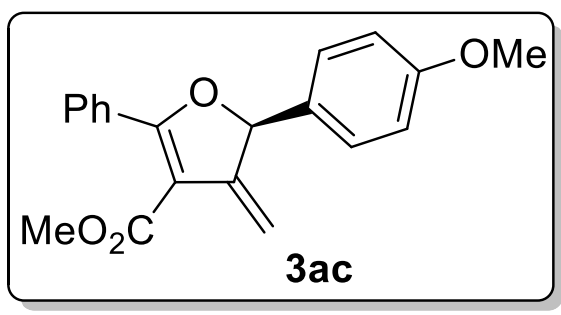
(3ac). Pale yellow solid (90 mg, 93\% yield) was obtained after purification with column chromatography on silica gel (hexanes/ethyl acetate, 40/1). M.p.: 64-66 ${ }^{\circ} \mathrm{C}$. 96\% ee was determined by chiral HPLC (Chiralpak AD-H, $n$-hexane/ $i$-PrOH $=$ 90/10, $0.8 \mathrm{~mL} / \mathrm{min}, 254 \mathrm{~nm}, 40^{\circ} \mathrm{C}$ ): $\mathrm{t}_{\mathrm{R}}$ (major) $=16.8 \mathrm{~min}, \mathrm{t}_{\mathrm{R}}$ $($ minor $)=10.5 \mathrm{~min} .[\alpha]_{\mathrm{D}}{ }^{26}=-151.9\left(c 1.13, \mathrm{CH}_{2} \mathrm{Cl}_{2}\right) .{ }^{1} \mathrm{H}$ NMR $\left(400 \mathrm{MHz}\right.$, DMSO-d $\left.{ }^{6}\right): \delta$ 7.69-7.67 $(\mathrm{m}, 2 \mathrm{H}), 7.52-7.43(\mathrm{~m}, 3 \mathrm{H}), 7.36-7.34(\mathrm{~m}, 2 \mathrm{H}), 7.00-6.98(\mathrm{~m}, 2 \mathrm{H}), 6.25(\mathrm{t}, J=2.9 \mathrm{~Hz}, 1 \mathrm{H}), 5.51$ (d, $J=3.3 \mathrm{~Hz}, 1 \mathrm{H}), 4.61(\mathrm{~d}, J=2.7 \mathrm{~Hz}, 1 \mathrm{H}), 3.77(\mathrm{~s}, 3 \mathrm{H}), 3.65(\mathrm{~s}, 3 \mathrm{H}) ;{ }^{13} \mathrm{C} \mathrm{NMR}(101 \mathrm{MHz}$, DMSO-d 6 ): $\delta 170.4,164.4,160.1,148.9,132.3,131.4,130.2,129.4,129.0,128.4,114.6,106.5$, 
102.7, 87.2, 55.6, 51.4. HRMS calc. for $\mathrm{C}_{20} \mathrm{H}_{17} \mathrm{O}_{4}[\mathrm{M}-\mathrm{H}]: 321.1127$, found: 321.1130 .

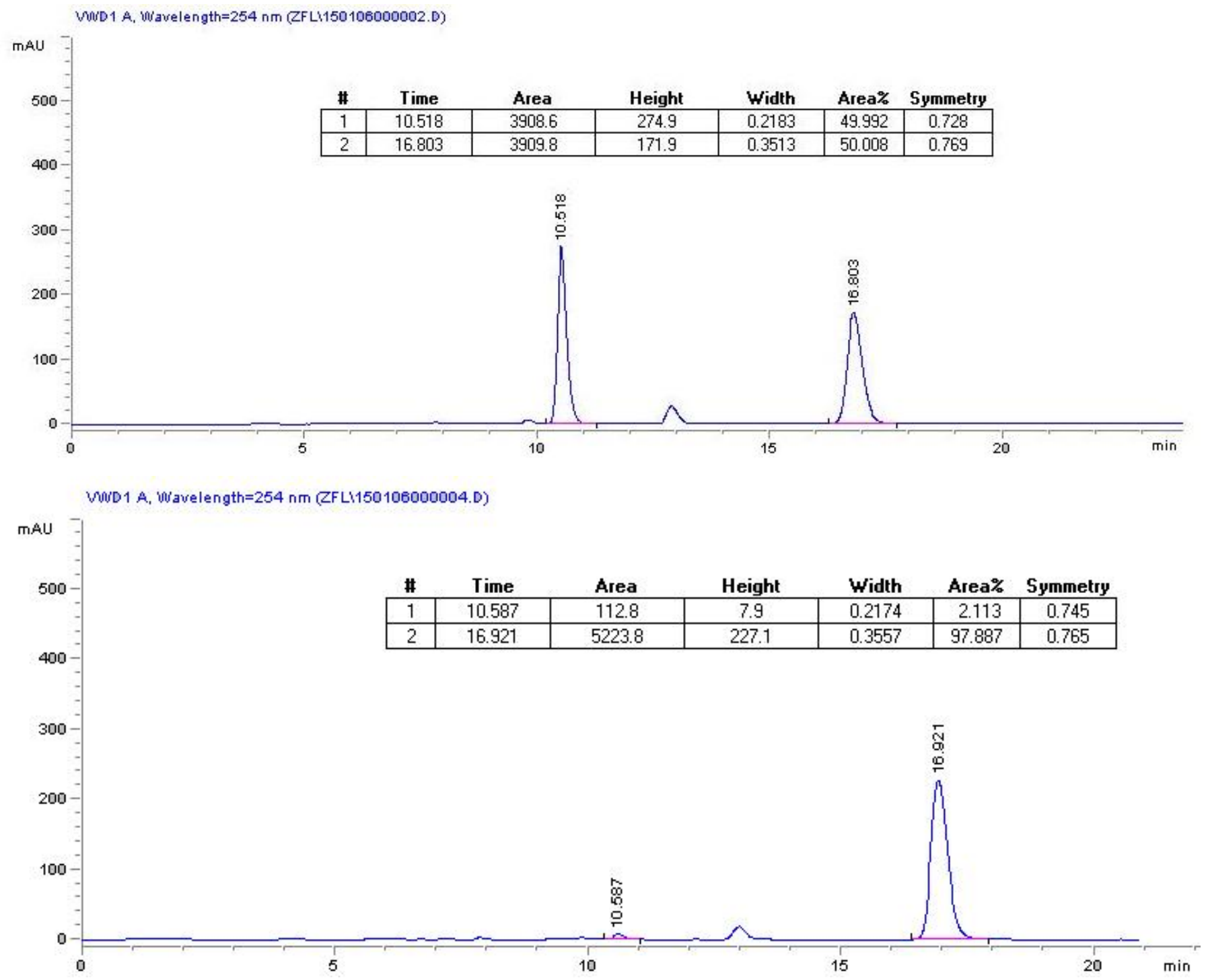

(S)-Methyl 4-methylene-2-phenyl-5-(4-(trifluoromethyl)phenyl)-4,5-dihydrofuran-3-carboxy-

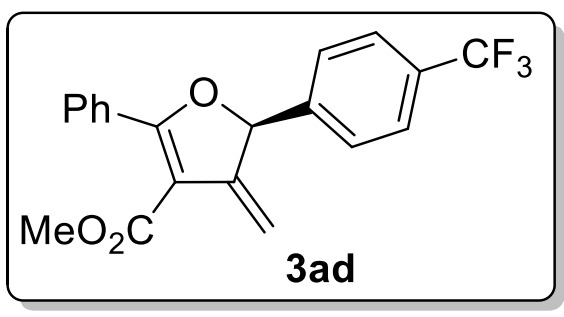
late (3ad). Pale yellow solid ( $89 \mathrm{mg}, 82 \%$ yield) was obtained after purification with column chromatography on silica gel (hexanes/ethyl acetate, 100/1). M.p.: $56-58{ }^{\circ} \mathrm{C} .96 \%$ ee was determined by chiral HPLC (Chiralpak AD-H, $n$-hexane $/ i-\mathrm{PrOH}=$ 90/10, $0.8 \mathrm{~mL} / \mathrm{min}, 254 \mathrm{~nm}, 40^{\circ} \mathrm{C}$ ): $\mathrm{t}_{\mathrm{R}}$ (major) $=9.5 \mathrm{~min}, \mathrm{t}_{\mathrm{R}}$ $($ minor $)=6.7 \mathrm{~min} .[\alpha]_{\mathrm{D}}{ }^{26}=-31.6\left(c 1.00, \mathrm{CH}_{2} \mathrm{Cl}_{2}\right) .{ }^{1} \mathrm{H}$ NMR $\left(400 \mathrm{MHz}, \mathrm{DMSO}-\mathrm{d}^{6}\right): \delta$ 7.82-7.65 $(\mathrm{m}$, $6 \mathrm{H}), 7.56-7.46(\mathrm{~m}, 3 \mathrm{H}), 6.45(\mathrm{t}, J=2.8 \mathrm{~Hz}, 1 \mathrm{H}), 5.51(\mathrm{~d}, J=3.2 \mathrm{~Hz}, 1 \mathrm{H}), 4.74(\mathrm{~d}, J=2.7 \mathrm{~Hz}, 1 \mathrm{H})$, 3.66 (s, 3H); ${ }^{13} \mathrm{C}$ NMR $(101 \mathrm{MHz}$, DMSO-d 6 ): $\delta$ 170.4, 164.2, 148.2, 144.7, 131.6, 129.8, 129.7 (q, $J=24.6 \mathrm{~Hz}$ ), 129.4, 128.5, 127.8, 126.3 (q, $J=3.8 \mathrm{~Hz}$ ), 124.0 (q, $J=162.4 \mathrm{~Hz}$ ), 106.5, 103.4, 85.9, 51.5; ${ }^{19} \mathrm{~F}$ NMR (376 MHz, DMSO-d $)$ : $\delta$-61.2. HRMS calc. for $\mathrm{C}_{20} \mathrm{H}_{15} \mathrm{~F}_{3} \mathrm{O}_{3} \mathrm{Na}[\mathrm{M}+\mathrm{Na}]^{+}$: 
383.0871, found: 383.0873 .

Woid A, Wiavelength $=254 \mathrm{~nm}$ (ZFLi141217000003.D)

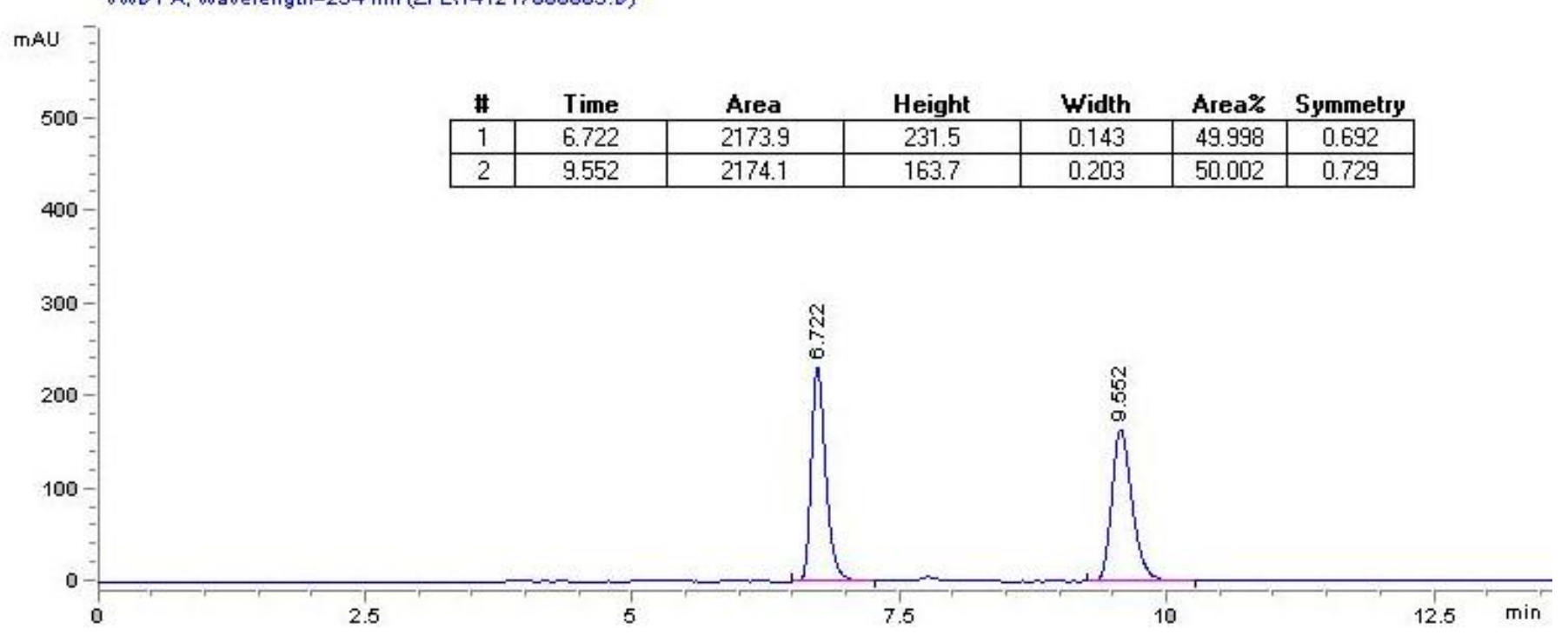

WuD $1 \mathrm{~A}$, Wiavelength $=254 \mathrm{~nm}$ (ZFLi141217000008.D)

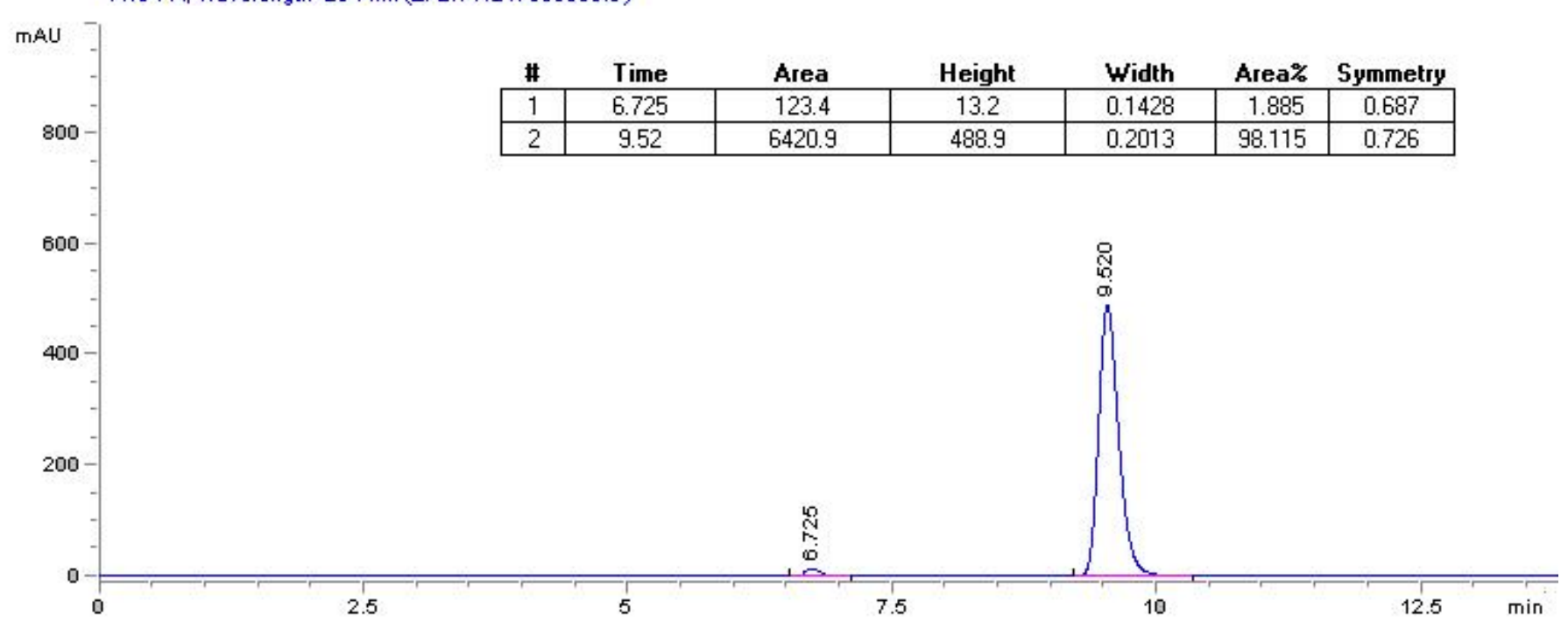

(S)-Methyl 5-(4-fluorophenyl)-4-methylene-2-phenyl-4,5-dihydrofuran-3-carboxylate (3ae).

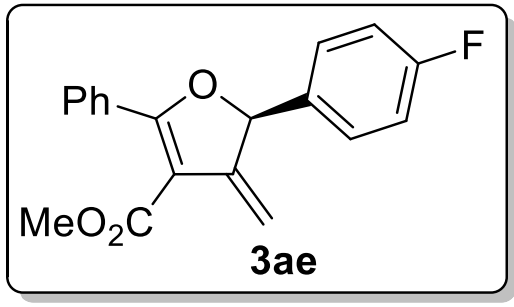

Yellow oil (87 mg, 93\% yield) was obtained after purification with column chromatography on silica gel (hexanes/ethyl acetate, 100/1). 97\% ee was determined by chiral HPLC (Chiralpak AD-H, $n$-hexane $/ \mathrm{i}-\mathrm{PrOH}=90 / 10,0.8 \mathrm{~mL} / \mathrm{min}, 254 \mathrm{~nm}, 40^{\circ} \mathrm{C}$ ): $\mathrm{tR}_{\mathrm{R}}($ major $)=$ $11.6 \mathrm{~min}, \mathrm{tR}_{\mathrm{R}}$ (minor) $=7.9 \mathrm{~min} .[\alpha]_{\mathrm{D}^{20}}=-122.1\left(\mathrm{c} \mathrm{1.01}, \mathrm{CH}_{2} \mathrm{Cl}_{2}\right) .{ }^{1} \mathrm{H}$

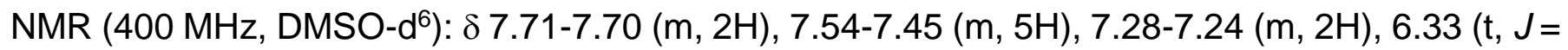
$2.8 \mathrm{~Hz}, 1 \mathrm{H}), 5.52(\mathrm{~d}, J=3.2 \mathrm{~Hz}, 1 \mathrm{H}), 4.66(\mathrm{~d}, J=2.7 \mathrm{~Hz}, 1 \mathrm{H}), 3.66(\mathrm{~s}, 3 \mathrm{H}) ;{ }^{13} \mathrm{C} N \mathrm{NMR}(101 \mathrm{MHz}$, DMSO-d $\left.{ }^{6}\right): \delta 170.3,164.3,162.7(d, J=245.0 \mathrm{~Hz}), 148.6,136.6(\mathrm{~d}, J=3.0 \mathrm{~Hz}), 131.5,130.0$, $129.5(\mathrm{~d}, J=8.5 \mathrm{~Hz}), 129.4,128.4,116.2(\mathrm{~d}, J=21.7 \mathrm{~Hz}), 106.5,103.1,86.4,51.5 ;{ }^{19} \mathrm{~F}$ NMR $(376$ 
$\mathrm{MHz}$, DMSO-d $\left.{ }^{6}\right): \delta$-113.2. HRMS calc. for $\mathrm{C}_{19} \mathrm{H}_{15} \mathrm{FO}_{3} \mathrm{Na}[\mathrm{M}+\mathrm{Na}]^{+}:$333.0903, found: 333.0908.

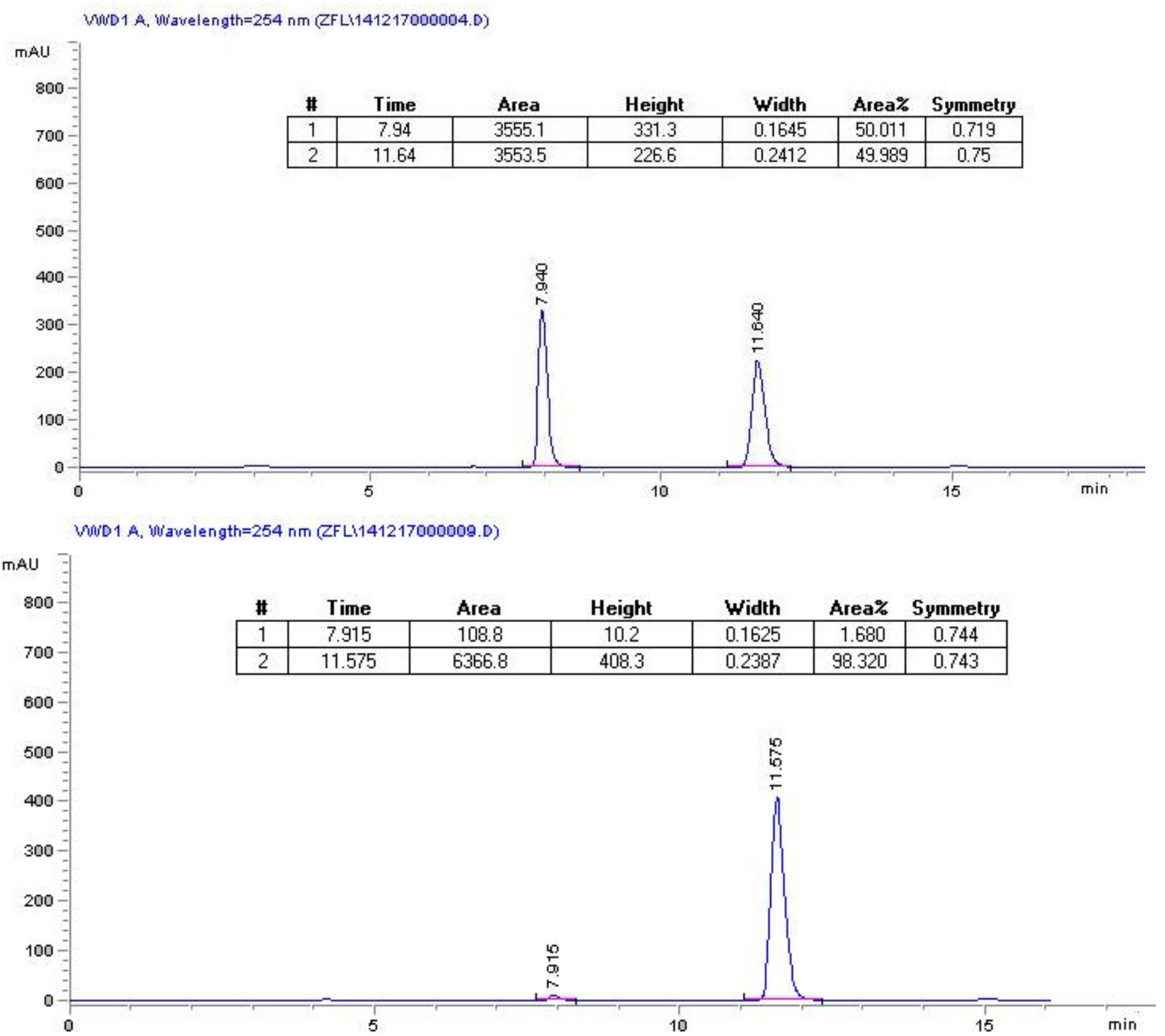

(S)-Methyl 4-(4-bromophenyl)-5-methylene-2-phenyl-4,5-dihydrofuran-3-carboxylate (3af).

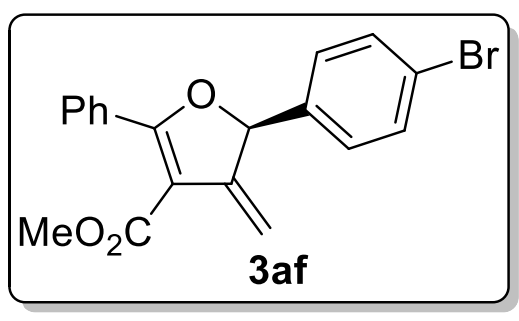

Pale yellow solid (101 $\mathrm{mg}, 91 \%$ yield) was obtained after purification with column chromatography on silica gel (hexanes/ethyl acetate, 100/1). M.p.: 71-73 C. 95\% ee was determined by chiral HPLC (Chiralpak AD-H, $n$-hexane/i-PrOH = 95/5, $\left.0.8 \mathrm{~mL} / \min , 254 \mathrm{~nm}, 40^{\circ} \mathrm{C}\right): \mathrm{t}_{\mathrm{R}}$ (major) = $17.7 \mathrm{~min}, \mathrm{t}_{\mathrm{R}}$ (minor) $=10.7 \mathrm{~min} .[\alpha]_{\mathrm{D}^{23}}=-94.3\left(c\right.$ 1.02, $\left.\mathrm{CH}_{2} \mathrm{Cl}_{2}\right) .{ }^{1} \mathrm{H}$ NMR $\left(400 \mathrm{MHz}, \mathrm{DMSO}-\mathrm{d}^{6}\right): \delta 7.72-7.63(\mathrm{~m}, 4 \mathrm{H})$, 7.54-7.38 (m, 5H), $6.31(\mathrm{~s}, 1 \mathrm{H}), 5.51(\mathrm{~d}, J=3.1 \mathrm{~Hz}, 1 \mathrm{H}), 4.69(\mathrm{~d}, J=2.4 \mathrm{~Hz}, 1 \mathrm{H}), 3.66(\mathrm{~s}, 3 \mathrm{H}) ;{ }^{13} \mathrm{C}$ NMR (101 MHz, DMSO-d6): $\delta$ 170.3, 164.3, 148.3, 139.6, 132.3, 131.5, 130.0, 129.4, 129.3, 
128.4, 122.5, 106.5, 103.2, 86.2, 51.5. HRMS calc. for $\mathrm{C}_{19} \mathrm{H}_{14} \mathrm{BrO}_{3}[\mathrm{M}-\mathrm{H}]: 369.0126$, found: 369.0123 .

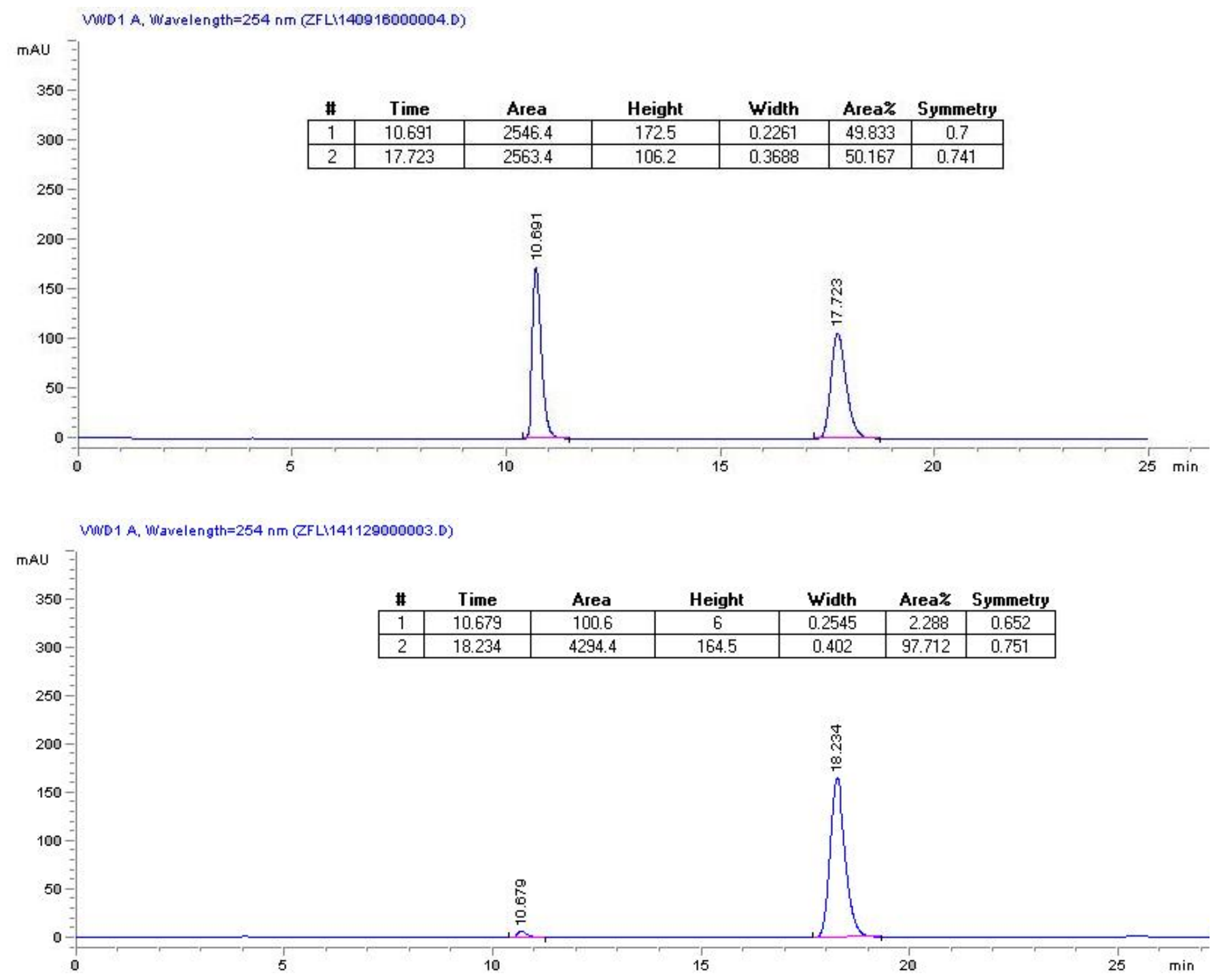

(S)-Methyl 5-(4-chlorophenyl)-4-methylene-2-phenyl-4,5-dihydrofuran-3-carboxylat (3ag).

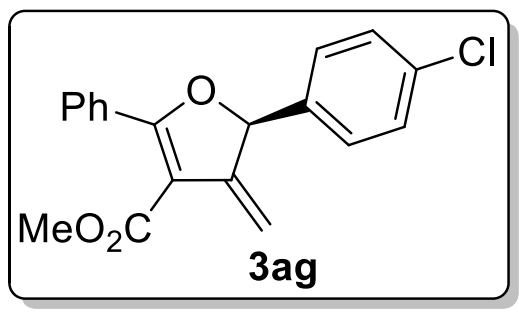

Yellow oil ( $86 \mathrm{mg}, 88 \%$ yield) was obtained after purification with column chromatography on silica gel (hexanes/ethyl acetate, 100/1). 96\% ee was determined by chiral HPLC (Chiralpak AD-H, $n$-hexane $/ \mathrm{i}-\mathrm{PrOH}=70 / 30,0.8 \mathrm{~mL} / \mathrm{min}, 254 \mathrm{~nm}, 40^{\circ} \mathrm{C}$ ): $\mathrm{tR}_{\mathrm{R}}$ (major) $=$ $8.9 \mathrm{~min}, \mathrm{tR}_{\mathrm{R}}$ (minor) $=6.3 \mathrm{~min} .[\alpha]_{\mathrm{D}}{ }^{25}=-115.3\left(c 1.11, \mathrm{CH}_{2} \mathrm{Cl}_{2}\right) .{ }^{1} \mathrm{H}$ NMR (400 MHz, DMSO-d $)$ : $\delta$ 7.72-7.70 (m, 2H), 7.52-7.44 (m, 7H), $6.33(\mathrm{t}, J=2.9 \mathrm{~Hz}, 1 \mathrm{H}), 5.52$ $(\mathrm{d}, J=3.2 \mathrm{~Hz}, 1 \mathrm{H}), 4.68(\mathrm{~d}, J=2.7 \mathrm{~Hz}, 1 \mathrm{H}), 3.66(\mathrm{~s}, 3 \mathrm{H}) ;{ }^{13} \mathrm{C}$ NMR $(101 \mathrm{MHz}$, DMSO-d $): \delta 170.4$, 164.3, 148.4, 139.2, 133.9, 131.5, 130.0, 129.4, 129.3, 129.1, 128.4, 106.5, 103.2, 86.2, 51.5. HRMS calc. for $\mathrm{C}_{19} \mathrm{H}_{15} \mathrm{ClO}_{3} \mathrm{Na}[\mathrm{M}+\mathrm{Na}]^{+}: 349.0607$, found: 349.0598 . 
Wio1 A, Wavelength=254 nm (ZFL:141204000007.D)

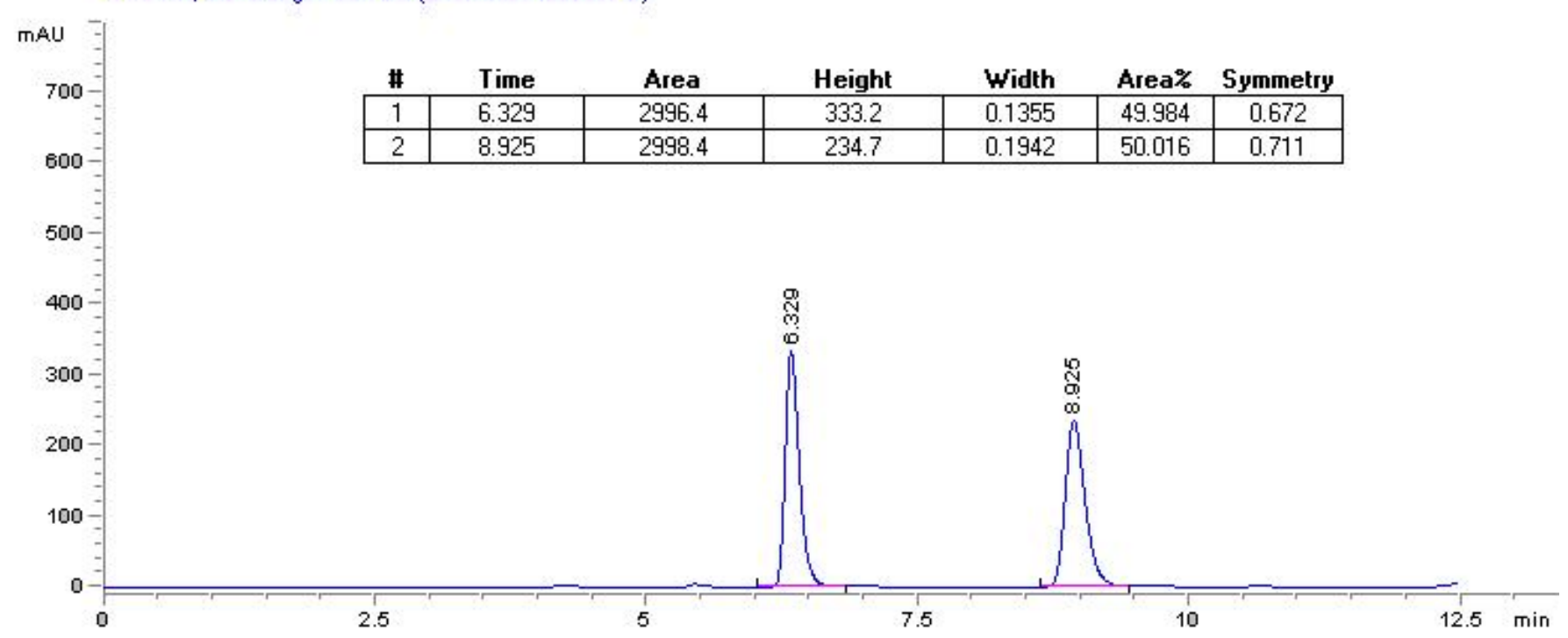

WidD1 A, wavelength $=254 \mathrm{~nm}$ (ZFLi141204000008.D)

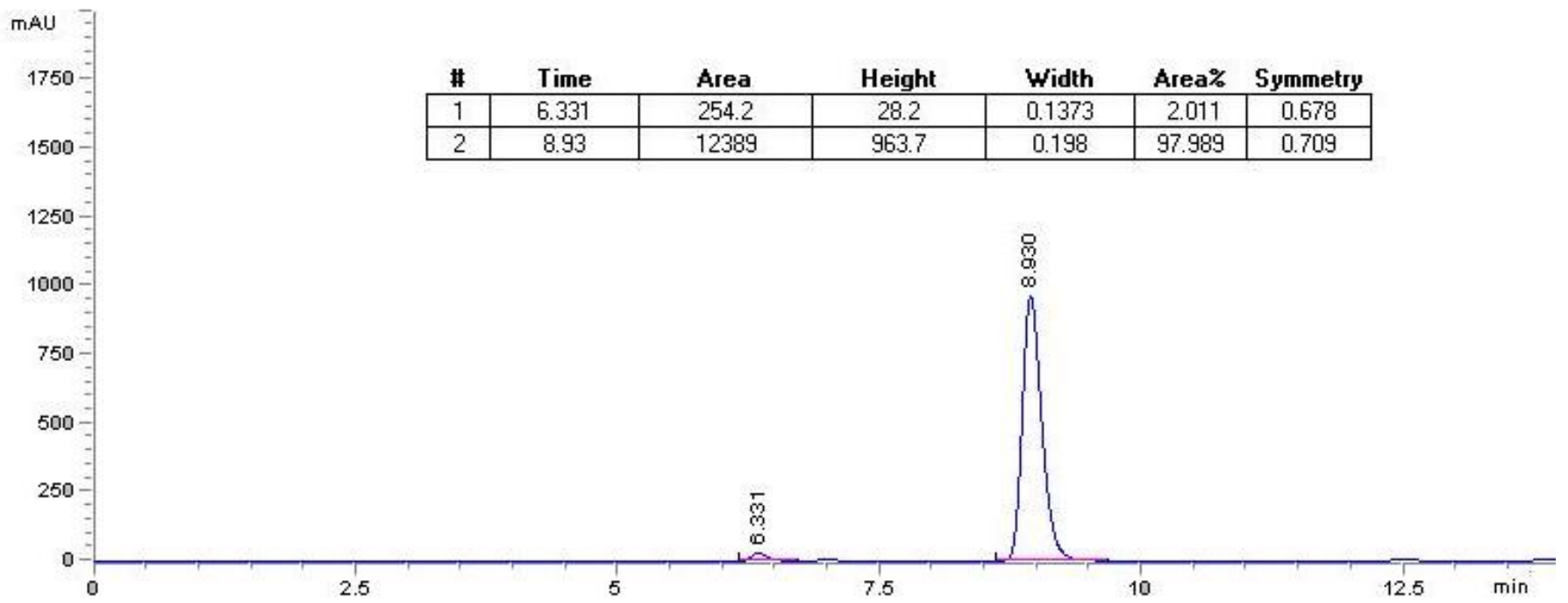

(S)-Methyl 5-(3-chlorophenyl)-4-methylene-2-phenyl-4,5-dihydrofuran-3-carboxylate (3ah).

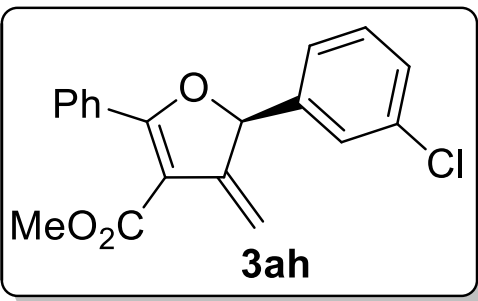

Yellow oil (86 mg, 88\% yield) was obtained after purification with column chromatography on silica gel (hexanes/ethyl acetate, 100/1). 97\% ee was determined by chiral HPLC (Chiralpak AD-H, $n$-hexane $\left./ \mathrm{i}-\mathrm{PrOH}=90 / 10,0.8 \mathrm{~mL} / \mathrm{min}, 254 \mathrm{~nm}, 40^{\circ} \mathrm{C}\right): \mathrm{t}_{\mathrm{R}}($ major$)=$ $10.0 \mathrm{~min}, \mathrm{t}_{\mathrm{R}}($ minor $)=7.3 \mathrm{~min} .[\alpha]_{\mathrm{D}}{ }^{25}=-124.4\left(c 1.12, \mathrm{CH}_{2} \mathrm{Cl}_{2}\right) .{ }^{1} \mathrm{H}$

NMR (400 MHz, DMSO-d6): $\delta$ 7.73-7.71 (m, 2H), 7.55-7.38 (m, 7H), $6.34(\mathrm{t}, \mathrm{J}=2.9 \mathrm{~Hz}, 1 \mathrm{H}), 5.53$ $(\mathrm{d}, J=3.2 \mathrm{~Hz}, 1 \mathrm{H}), 4.72(\mathrm{~d}, J=2.7 \mathrm{~Hz}, 1 \mathrm{H}), 3.66(\mathrm{~s}, 3 \mathrm{H}) ;{ }^{13} \mathrm{C}$ NMR $\left(101 \mathrm{MHz}, \mathrm{DMSO}-\mathrm{d}^{6}\right): \delta 170.3$, 164.2, 148.2, 142.6, 133.9, 131.6, 131.3, 129.9, 129.4, 129.1, 128.5, 127.0, 125.8, 106.5, 103.3, 86.0, 51.5. HRMS calc. for $\mathrm{C}_{19} \mathrm{H}_{15} \mathrm{ClO}_{3} \mathrm{Na}[\mathrm{M}+\mathrm{Na}]^{+}: 349.0607$, found: 349.0599. 


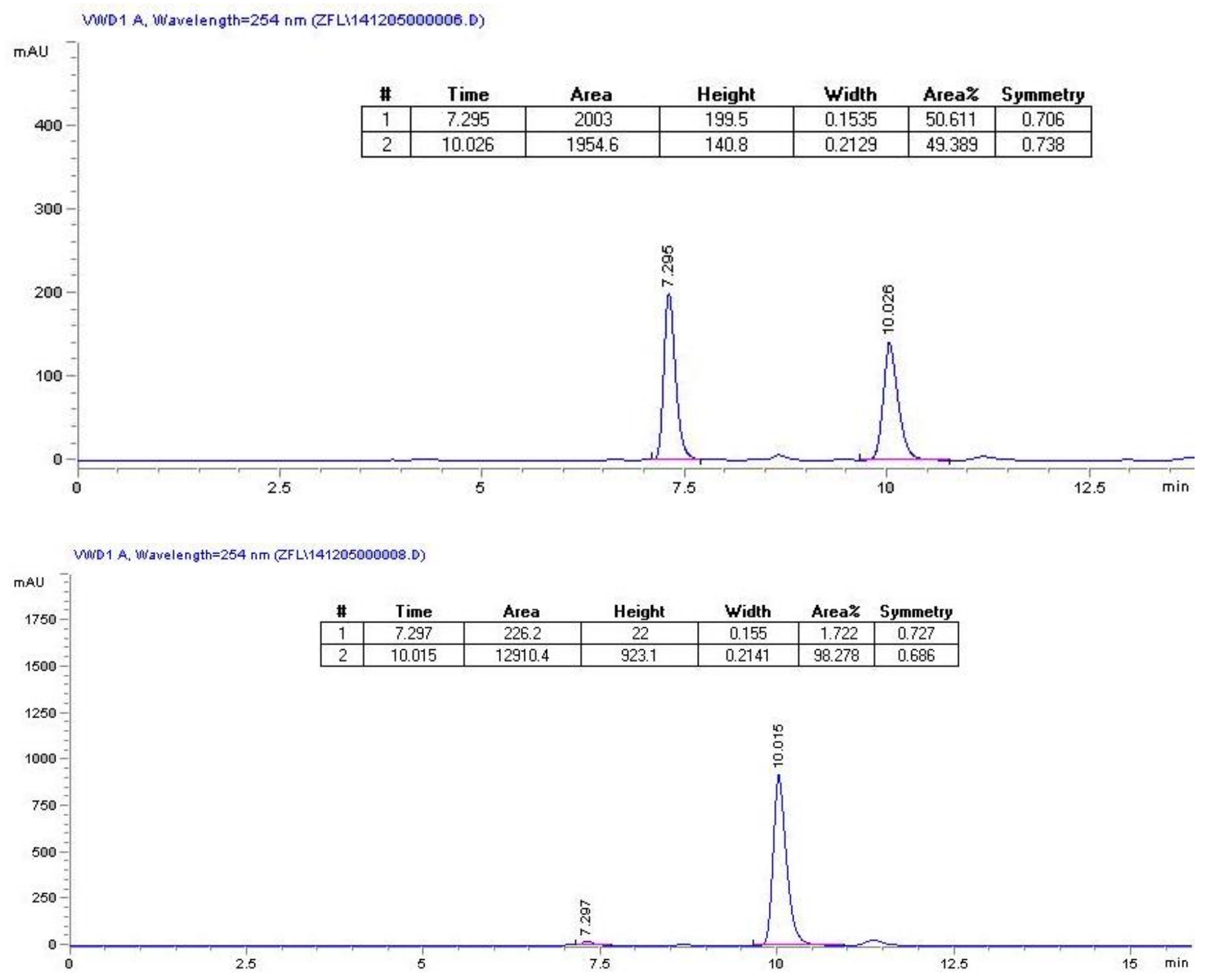

(S)-Methyl 5-(2-chlorophenyl)-4-methylene-2-phenyl-4,5-dihydrofuran-3-carboxylate (3ai).

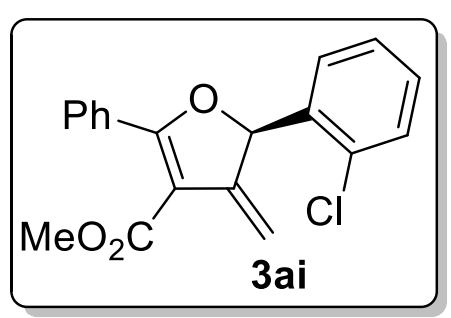

Yellow oil (69 $\mathrm{mg}, 70 \%$ yield) was obtained after purification with column chromatography on silica gel (hexanes/ethyl acetate, 100/1). 98\% ee was determined by chiral HPLC (Chiralpak AD-H, $n$-hexane $/$ - $\left.\mathrm{PrOH}=90 / 10,0.8 \mathrm{~mL} / \mathrm{min}, 254 \mathrm{~nm}, 40^{\circ} \mathrm{C}\right): \mathrm{t}_{\mathrm{R}}$ (major) $=$ $11.2 \mathrm{~min}, \mathrm{t}_{\mathrm{R}}$ (minor) $=7.7 \mathrm{~min} .[\alpha]_{\mathrm{D}}{ }^{21}=-24.4\left(c 1.16, \mathrm{CH}_{2} \mathrm{Cl}_{2}\right) .{ }^{1} \mathrm{H} \mathrm{NMR}$ (400 MHz, DMSO-d 6 ): $\delta$ 7.72-7.69 (m, 2H), 7.57-7.41 (m, 7H), $6.60(\mathrm{t}, J=3.1 \mathrm{~Hz}, 1 \mathrm{H}), 5.51(\mathrm{~d}, J=$ $3.4 \mathrm{~Hz}, 1 \mathrm{H}$ ), 4.68 (d, $J=2.9 \mathrm{~Hz}, 1 \mathrm{H}$ ), $3.66(\mathrm{~s}, 3 \mathrm{H}) ;{ }^{13} \mathrm{C}$ NMR (101 MHz, DMSO-d 6 ): $\delta$ 170.5, 164.2, 147.7, 137.0, 132.8, 131.5, 131.1, 130.5, 130.0, 129.9, 129.4, 128.4, 128.3, 106.8, 102.6, 84.5, 51.5. HRMS calc. for $\mathrm{C}_{19} \mathrm{H}_{14} \mathrm{ClO}_{3}[\mathrm{M}-\mathrm{H}]=325.0631$, found: 325.0621 . 

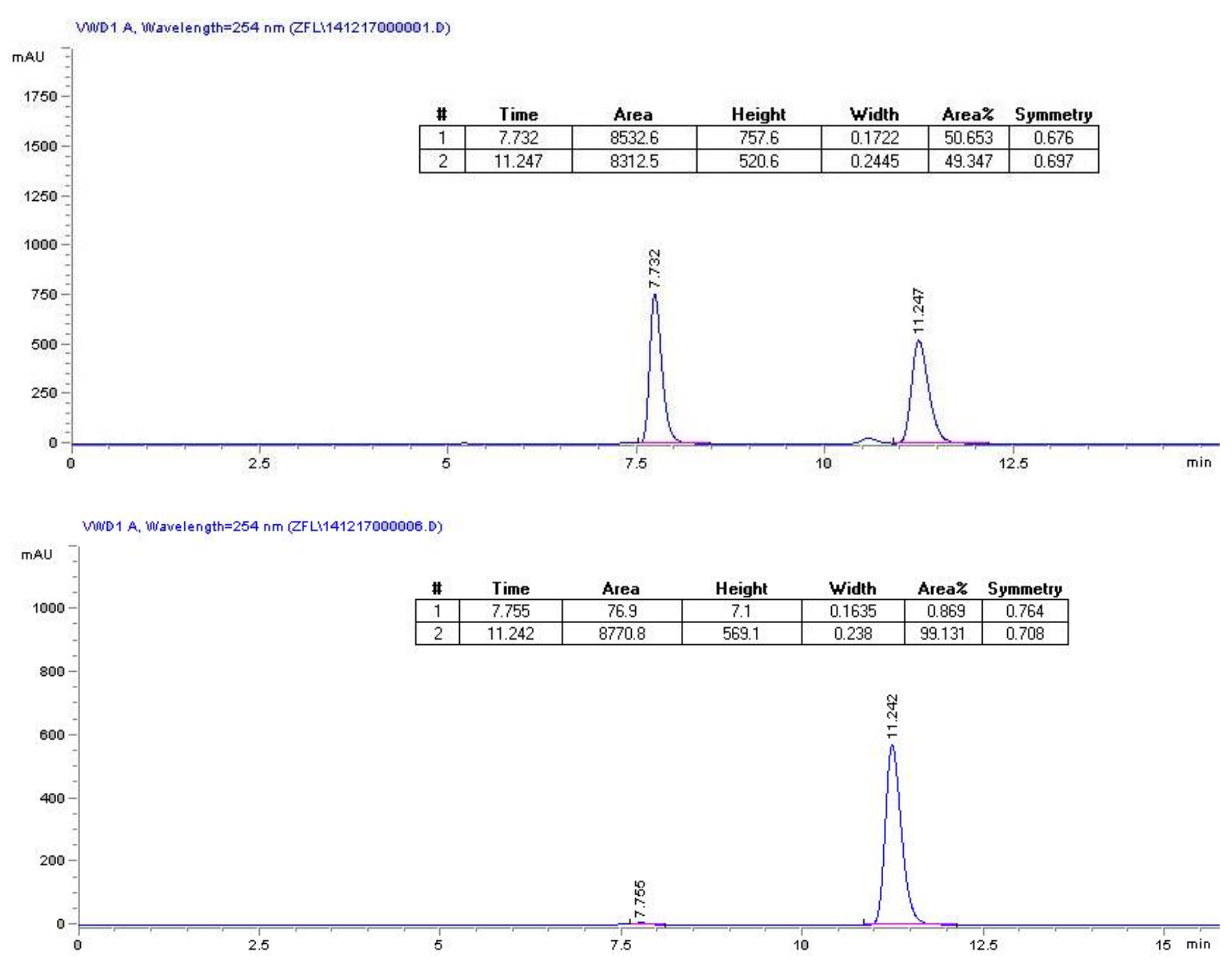

(S)-Methyl 4-methylene-5-(naphthalen-2-yl)-2-phenyl-4,5-dihydrofuran-3-carboxylate (3aj).

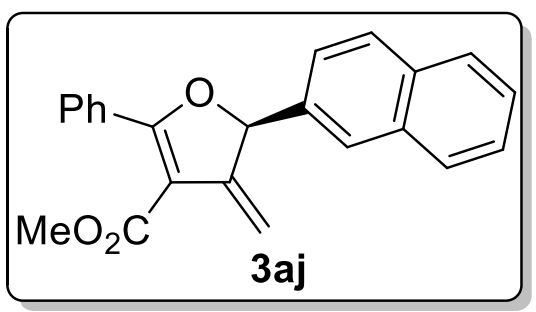
Pale yellow solid ( $88 \mathrm{mg}, 86 \%$ yield) was obtained after purification with column chromatography on silica gel (hexanes/ethyl acetate, 40/1). M.p.: $134-136{ }^{\circ} \mathrm{C}$. 95\% ee was determined by chiral HPLC (Chiralpak AD-H, $n$-hexane/i-PrOH $=$ 90/10, $0.8 \mathrm{~mL} / \mathrm{min}, 254 \mathrm{~nm}, 40{ }^{\circ} \mathrm{C}$ ): tR (major) = $19.9 \mathrm{~min}, \mathrm{tR}_{\mathrm{R}}$ $($ minor $)=9.9$ min. $[\alpha]_{D^{25}}=-120.1\left(c 0.84, \mathrm{CH}_{2} \mathrm{Cl}_{2}\right) .{ }^{1} \mathrm{H}$ NMR $\left(400 \mathrm{MHz}, \mathrm{DMSO}-\mathrm{d}^{6}\right): \delta 8.00-7.92(\mathrm{~m}$, 4H), 7.76-7.75 (m, 2H), 7.56-7.46 (m, 6H), $6.48(\mathrm{t}, J=2.8 \mathrm{~Hz}, 1 \mathrm{H}), 5.55(\mathrm{~d}, J=3.2 \mathrm{~Hz}, 1 \mathrm{H}), 4.70$ (d, $J=2.6 \mathrm{~Hz}, 1 \mathrm{H}), 3.68(\mathrm{~s}, 3 \mathrm{H}) ;{ }^{13} \mathrm{C}$ NMR $(101 \mathrm{MHz}$, DMSO-d $): \delta$ 170.7, 164.4, 148.7, 137.5, 133.4, 133.1, 131.5, 130.1, 129.5, 129.3, 128.6, 128.5, 128.1, 127.1, 127.0, 126.7, 124.7, 106.7, 103.1, 87.4, 51.5. HRMS calc. for $\mathrm{C}_{23} \mathrm{H}_{18} \mathrm{O}_{3} \mathrm{Na}[\mathrm{M}+\mathrm{Na}]^{+}: 365.1154$, found: 365.1159 . 
mAl

\begin{tabular}{|c|c|c|c|c|c|c|}
\hline$\#$ & Time & Area & Height & width & Area\% & Symmetry \\
\hline 1 & 9.9 & 1659.8 & 123.2 & 0.2067 & 50.131 & 0.74 \\
\hline 2 & 19.918 & 1651.1 & 59.6 & 0.4279 & 49.869 & 0.784 \\
\hline
\end{tabular}

200
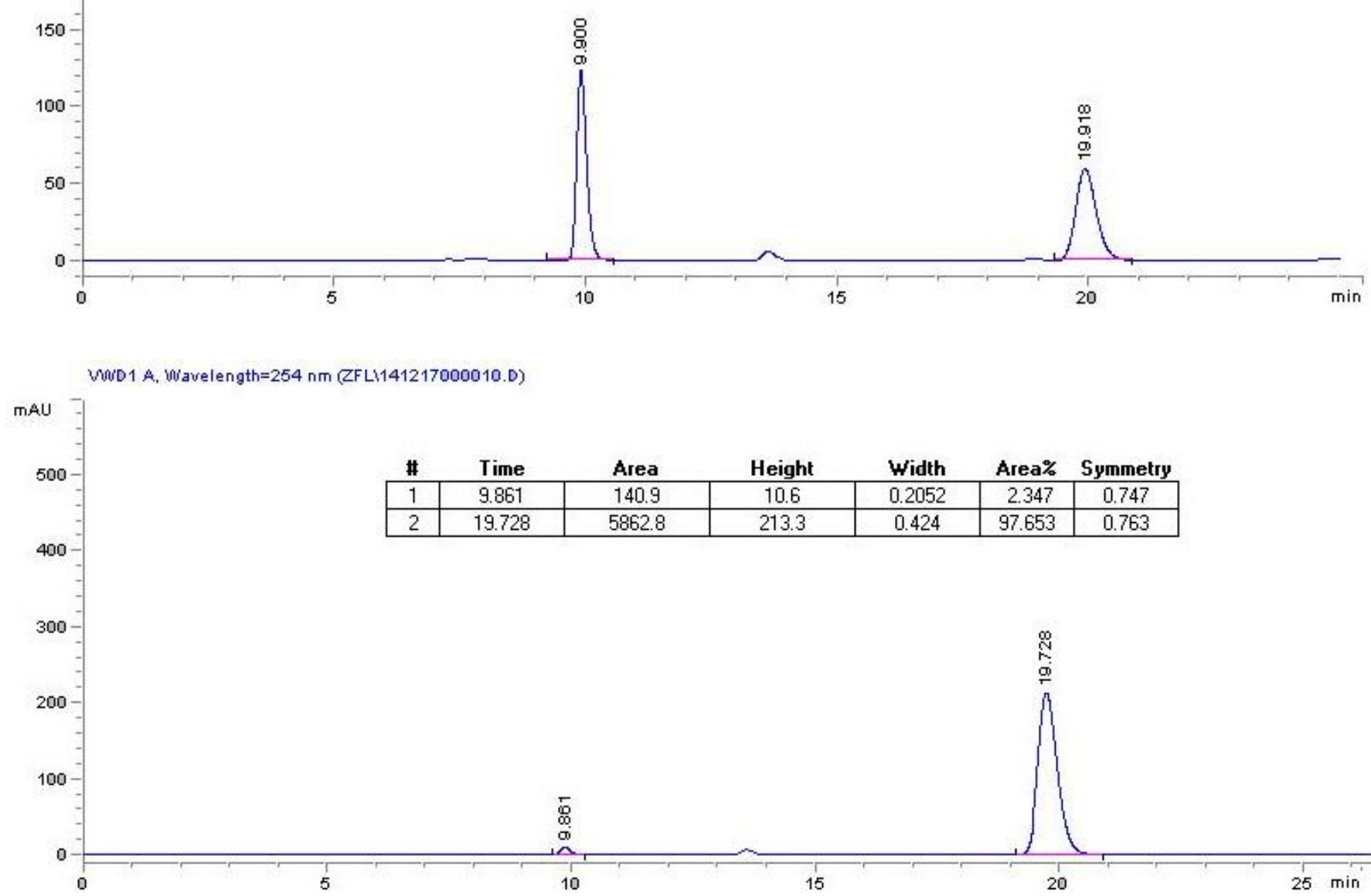

(S)-Methyl 4-methylene-2-phenyl-5-(thiophen-2-yl)-4,5-dihydrofuran-3-carboxylate (3ak).

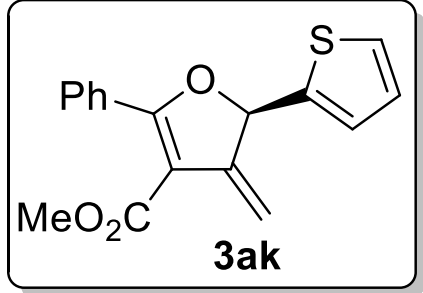

Pale yellow solid (78 $\mathrm{mg}, 87 \%$ yield) was obtained after purification with column chromatography on silica gel (hexanes/ethyl acetate, 100/1). M.p.: $43-45{ }^{\circ} \mathrm{C}$. $83 \%$ ee was determined by chiral HPLC (Chiralpak $\mathrm{AD}-\mathrm{H}, n$-hexane $/ \mathrm{i}-\mathrm{PrOH}=90 / 10,0.8 \mathrm{~mL} / \mathrm{min}, 254 \mathrm{~nm}, 40^{\circ} \mathrm{C}$ ): $\mathrm{t}_{\mathrm{R}}$ (major) $=12.2 \mathrm{~min}, \mathrm{t}_{\mathrm{R}}$ (minor) $=8.2 \mathrm{~min} .[\alpha]_{\mathrm{D}}{ }^{22}=-308.7\left(c \quad 0.90, \mathrm{CH}_{2} \mathrm{Cl}_{2}\right) .{ }^{1} \mathrm{H}$

NMR (400 MHz, DMSO-d $)$ : $\delta$ 7.67-7.62 (m, 3H), 7.52-7.44 (m, 3H), 7.34-7.33 (m, 1H), 7.10-7.08 $(\mathrm{m}, 1 \mathrm{H}), 6.64(\mathrm{t}, J=2.9 \mathrm{~Hz}, 1 \mathrm{H}), 5.60(\mathrm{~d}, J=3.2 \mathrm{~Hz}, 1 \mathrm{H}), 4.81(\mathrm{~d}, J=2.7 \mathrm{~Hz}, 1 \mathrm{H}), 3.66(\mathrm{~s}, 3 \mathrm{H})$; ${ }^{13} \mathrm{C}$ NMR (101 MHz, DMSO-d $): \delta$ 169.6, 164.2, 148.0, 142.7, 131.6, 130.0, 129.4, 128.5, 128.1, 128.0, 127.5, 106.5, 103.9, 82.7, 51.5. HRMS calc. for $\mathrm{C}_{17} \mathrm{H}_{14} \mathrm{O}_{3} \mathrm{SNa}[\mathrm{M}+\mathrm{Na}]^{+}: 321.0561$, found: 321.0562 . 

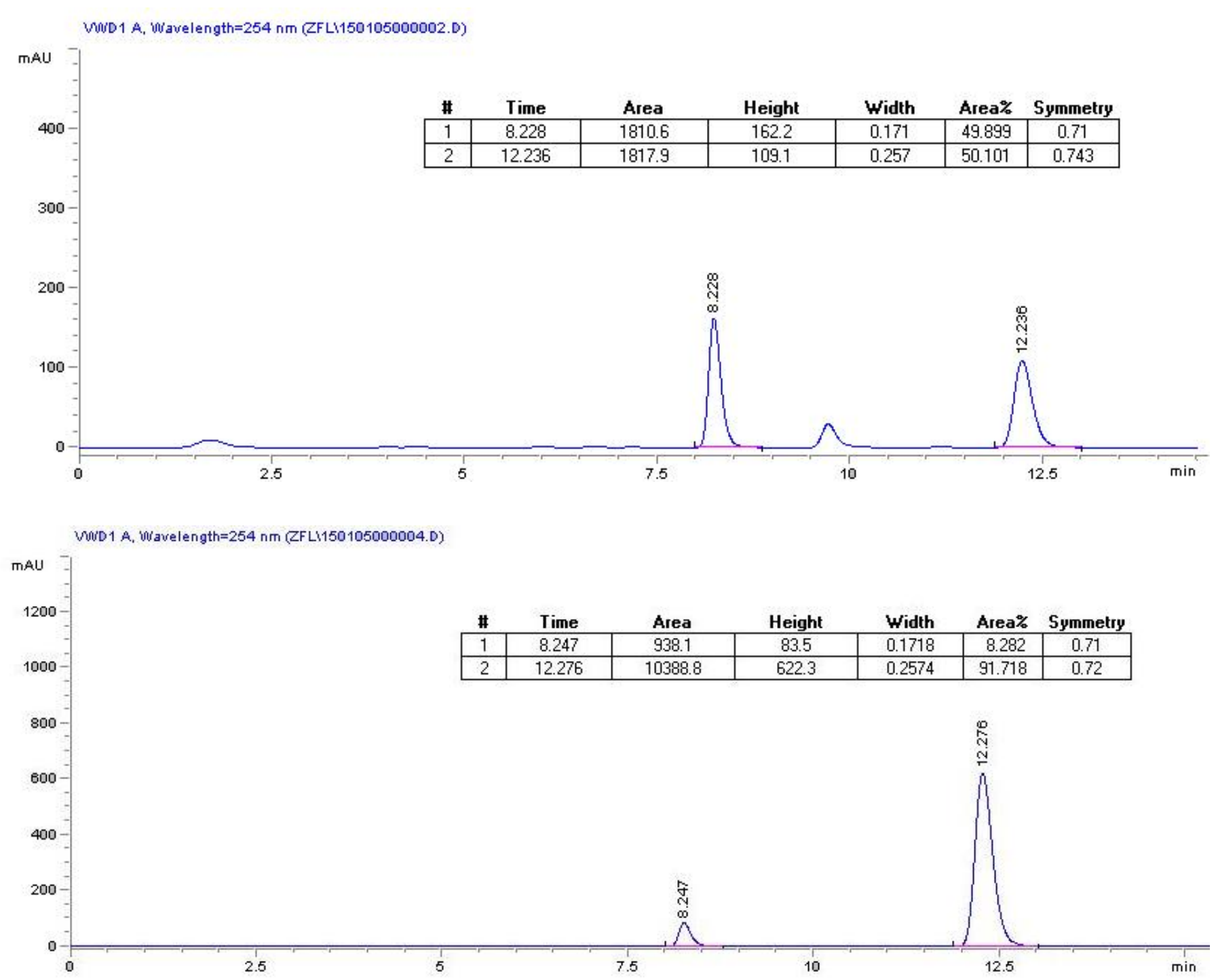

(S)-Methyl 5-methyl-4-methylene-2-phenyl-4,5-dihydrofuran-3-carboxylate (3al). Colorless

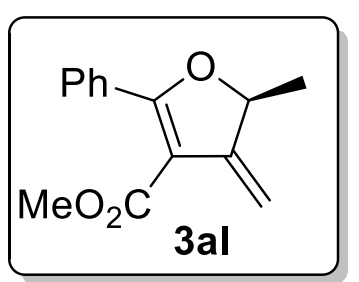

oil (59 mg, $85 \%$ yield) was obtained after purification with column chromatography on silica gel (hexanes/ethyl acetate, 100/1). $56 \%$ ee was determined by chiral HPLC (Chiralpak AD-H, $n$-hexane $/ i-\mathrm{PrOH}=90 / 10,0.8$ $\left.\mathrm{mL} / \mathrm{min}, 254 \mathrm{~nm}, 40^{\circ} \mathrm{C}\right): \mathrm{tR}_{\mathrm{R}}($ major $)=11.9 \mathrm{~min}, \mathrm{tR}_{\mathrm{R}}($ minor $)=11.0 \mathrm{~min} .[\alpha]_{\mathrm{D}^{18}}=$ -23.0 (c 0.62, $\mathrm{CH}_{2} \mathrm{Cl}_{2}$ ). ${ }^{1} \mathrm{H}$ NMR (400 MHz, DMSO-d $\left.\mathrm{d}^{6}\right): \delta$ 7.64-7.62 (m, 2H), 7.53-7.43 (m, 3H), $5.40(\mathrm{~d}, J=3.1 \mathrm{~Hz}, 1 \mathrm{H}), 5.36(\mathrm{qd}, J=6.4,3.1 \mathrm{~Hz}, 1 \mathrm{H}), 4.81(\mathrm{~d}, J=2.6 \mathrm{~Hz}, 1 \mathrm{H}), 3.62(\mathrm{~s}, 3 \mathrm{H}), 1.47$ (d, $J=6.4 \mathrm{~Hz}, 3 \mathrm{H}) ;{ }^{13} \mathrm{C}$ NMR (101 MHz, DMSO-d 6 ): $\delta 170.3,164.5,149.8,131.3,130.4,129.3$, 128.3, 106.1, 100.3, 82.8, 51.3, 22.2. HRMS calc. for $\mathrm{C}_{14} \mathrm{H}_{15} \mathrm{O}_{3}[\mathrm{M}+\mathrm{H}]^{+}: 231.1021$, found: 231.1014 . 


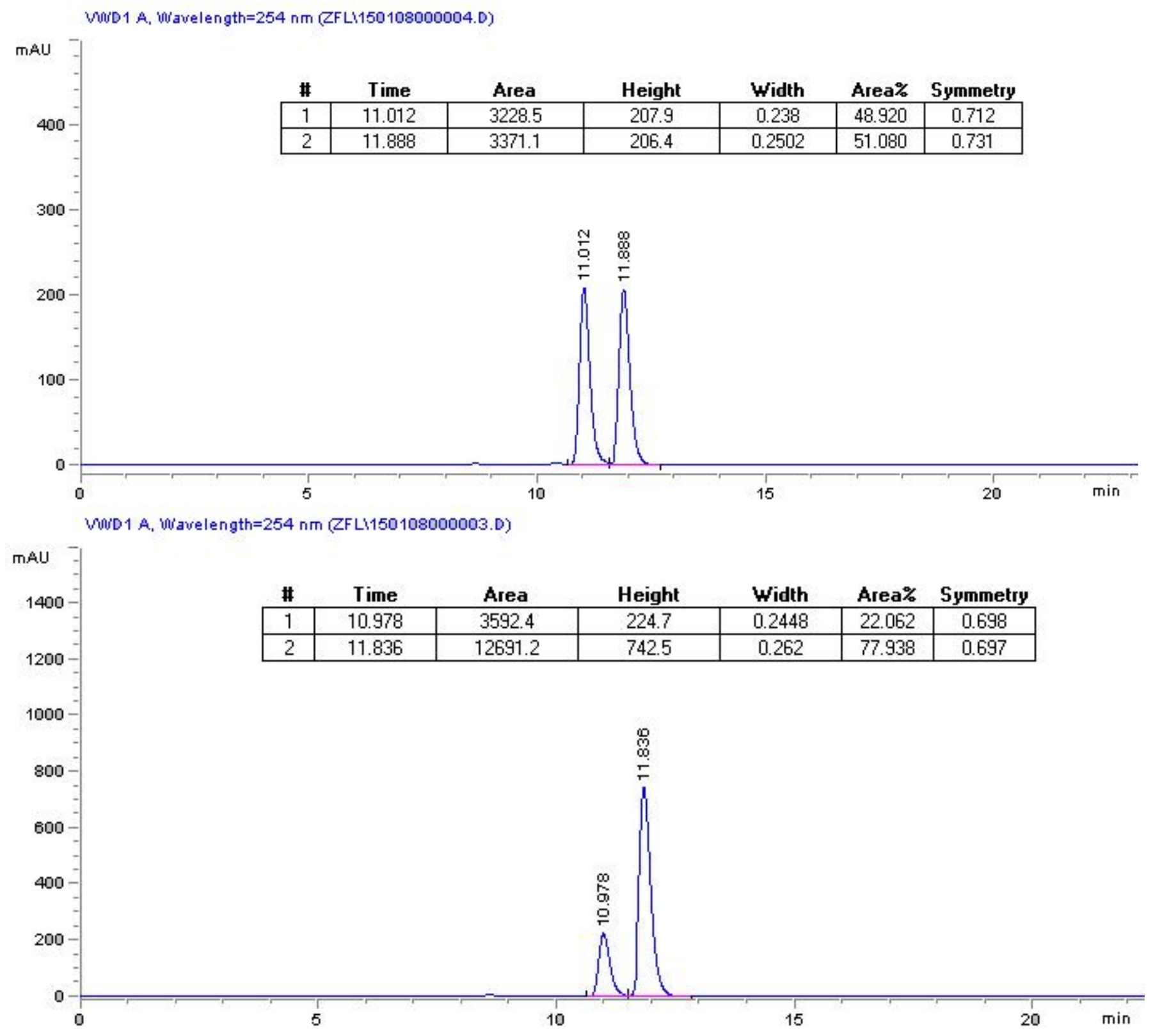

(S,E)-Methyl 4-benzylidene-2,5-diphenyl-4,5-dihydrofuran-3-carboxylate (3am). Yellow oil

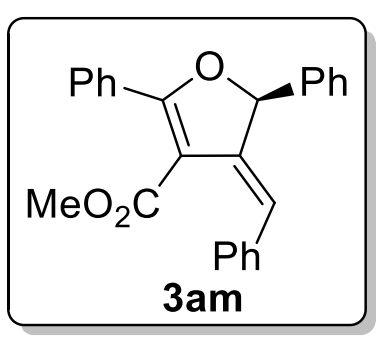

(97 mg, 88\% yield) was obtained after purification with column chromatography on silica gel (hexanes/ethyl acetate, 100/1), >95/5 d.r. was determined by ${ }^{1} \mathrm{H}$ NMR. $89 \%$ ee was determined by chiral HPLC (Chiralpak AD-H, $n$-hexane/i-PrOH = 90/10, $0.8 \mathrm{~mL} / \mathrm{min}, 254 \mathrm{~nm}, 40^{\circ} \mathrm{C}$ ): $\mathrm{t}_{\mathrm{R}}$ (major) $=18.1 \mathrm{~min}, \mathrm{tR}_{\mathrm{R}}$ (minor $)=15.5 \mathrm{~min} .[\alpha]_{\mathrm{D}}^{22}=-73.7\left(c 1.00, \mathrm{CH}_{2} \mathrm{Cl}_{2}\right)$. ${ }^{1} \mathrm{H}$ NMR $(400 \mathrm{MHz}$, DMSO-d6): $\delta$ 7.78-7.76 $(\mathrm{m}, 2 \mathrm{H}), 7.52-7.41(\mathrm{~m}, 8 \mathrm{H}), 7.30-7.16(\mathrm{~m}, 3 \mathrm{H})$, 7.07-7.05 (m, 2H), $6.43(\mathrm{~d}, J=2.8 \mathrm{~Hz}, 1 \mathrm{H}), 6.08(\mathrm{~d}, J=2.6 \mathrm{~Hz}, 1 \mathrm{H}), 3.11(\mathrm{~s}, 3 \mathrm{H}) ;{ }^{13} \mathrm{C}$ NMR $(101$ MHz, DMSO-d 6 ): $\delta$ 165.8, 165.7, 142.7, 140.6, 137.8, 131.5, 129.4, 129.4, 129.3, 129.0, 128.4, 
128.3, 127.9, 127.4, 126.9, 117.6, 108.2, 87.6, 51.6. HRMS calc. for $\mathrm{C}_{25} \mathrm{H}_{21} \mathrm{O}_{3}[\mathrm{M}+\mathrm{H}]^{+}:$369.1491, found: 369.1471 .

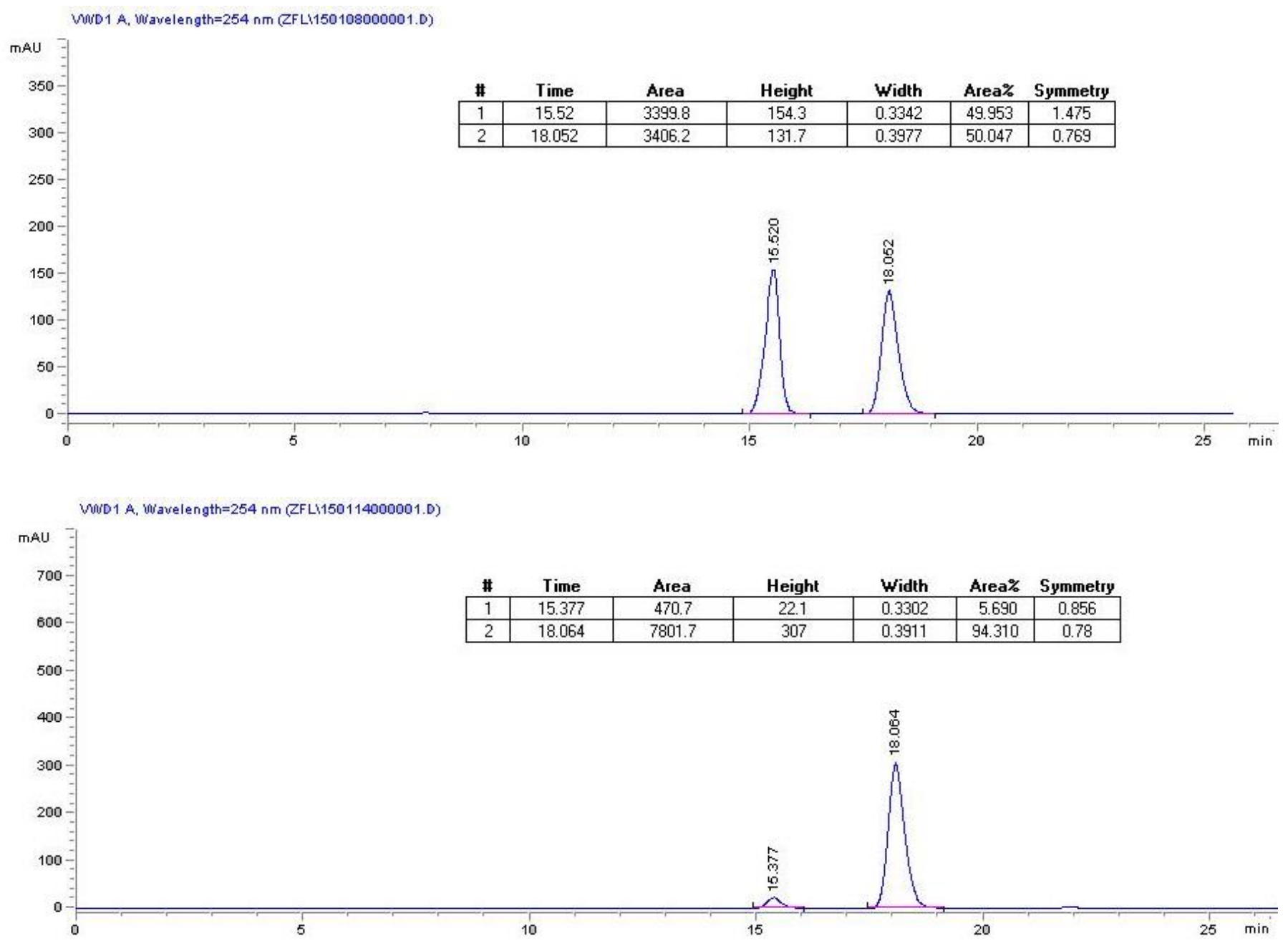

(S,E)-Methyl 4-pentylidene-5-phenyl-2-(p-tolyl)-4,5-dihydrofuran-3-carboxylate

(3an).

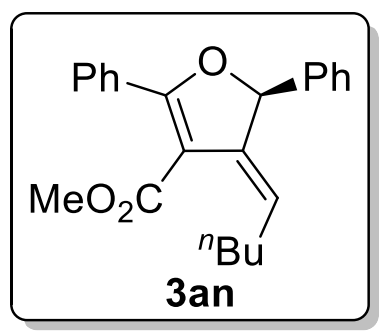

Yellow oil (86 $\mathrm{mg}, 82 \%$ yield) was obtained after purification with column chromatography on silica gel (hexanes/ethyl acetate, 100/1). 95\% ee was determined by chiral HPLC (Chiralpak AD-H, $n$-hexane/ $\mathrm{i}-\mathrm{PrOH}=97 / 3,0.8$ $\left.\mathrm{mL} / \mathrm{min}, 254 \mathrm{~nm}, 40^{\circ} \mathrm{C}\right): \mathrm{t}_{\mathrm{R}}$ (major) $=14.4 \mathrm{~min}$, $\mathrm{t}_{\mathrm{R}}($ minor $)=10.9 \mathrm{~min} .[\alpha]_{\mathrm{D}}{ }^{24}$ $=-60.4\left(c 0.80, \mathrm{CH}_{2} \mathrm{Cl}_{2}\right) .{ }^{1} \mathrm{H}$ NMR $(400 \mathrm{MHz}$, DMSO-d $)$ for 92:8 mixture of geometric isomers: $\delta 7.61-7.58(\mathrm{~m}, 2 \mathrm{H}), 7.50-7.37(\mathrm{~m}, 8 \mathrm{H}) 6.17(\mathrm{dd}, J=4.5,2.1 \mathrm{~Hz}, 1 \mathrm{H}), 4.91$ (td, $J=7.7,2.6 \mathrm{~Hz}, 1 \mathrm{H}), 3.74(\mathrm{~s}, 3 \mathrm{H}), 2.04-1.92(\mathrm{~m}, 2 \mathrm{H}), 1.26-1.20(\mathrm{~m}, 4 \mathrm{H}), 0.82(\mathrm{t}, J=7.1 \mathrm{~Hz}, 3 \mathrm{H})$; ${ }^{13} \mathrm{C}$ NMR (101 MHz, DMSO-d $): \delta$ 167.1, 162.3, 141.1, 140.5, 131.0, 129.6, 129.2, 129.1, 129.0, $127.4,127.1,118.9,108.0,86.8,52.5,32.0,27.7,22.2,14.1$. HRMS calc. for $\mathrm{C}_{23} \mathrm{H}_{25} \mathrm{O}_{3}[\mathrm{M}+\mathrm{H}]^{+}$: 349.1804 , found: 349.1806 . 


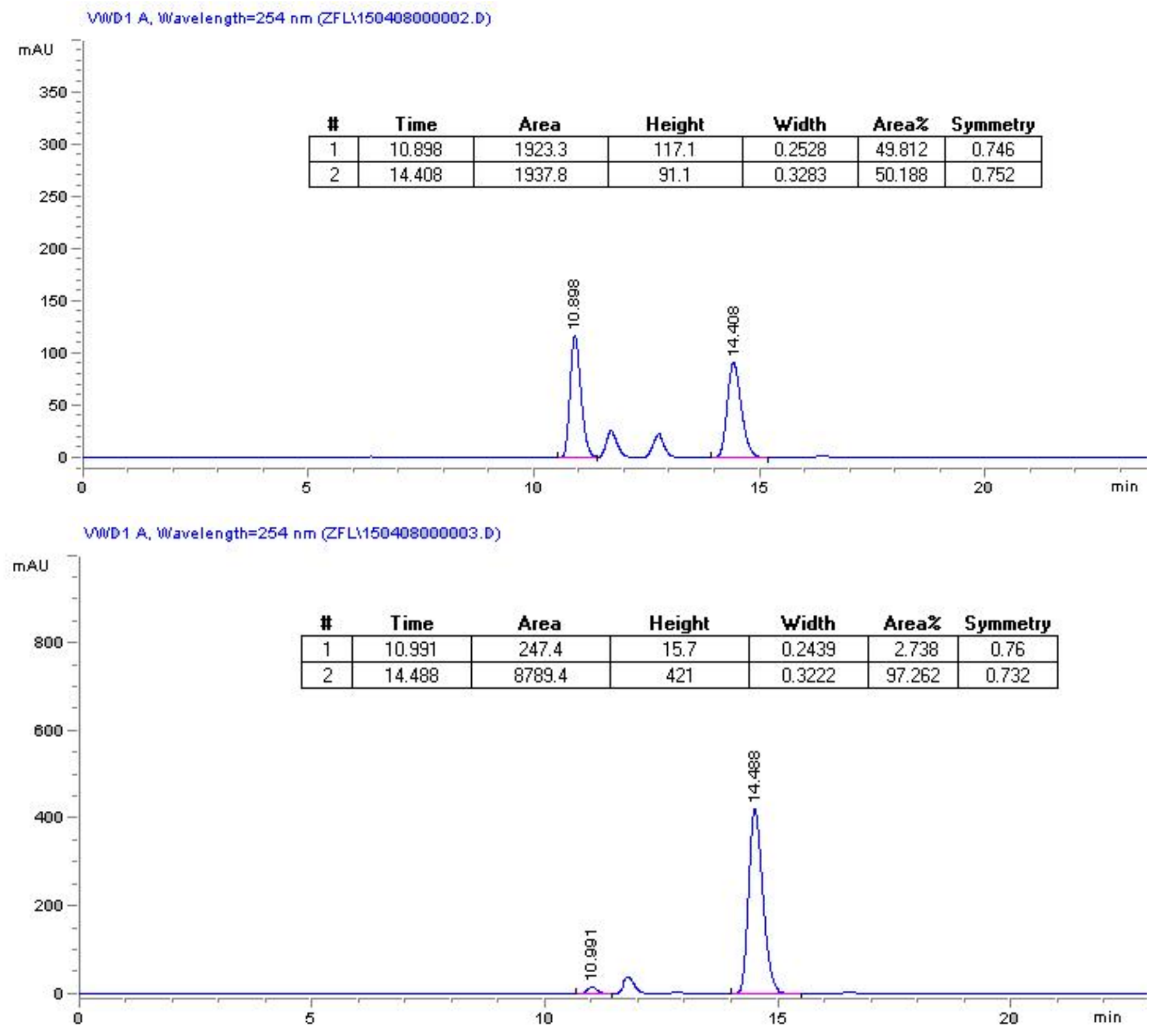

(S)-Methyl 2-(4-bromophenyl)-4-methylene-5-(naphthalen-2-yl)-4,5-dihydrofuran-3-carbox-

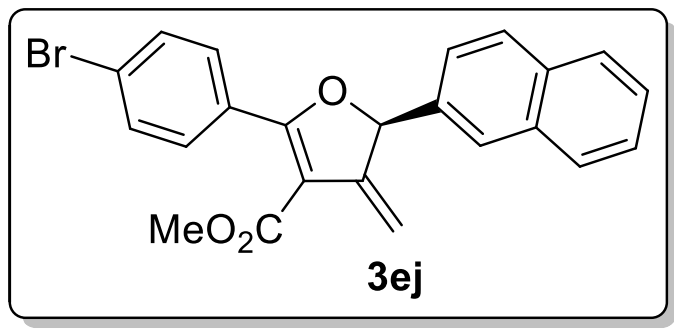
ylate (3ej). Pale yellow solid (116 $\mathrm{mg}, 92 \%$ yield) was obtained after purification with column chromatography on silica gel (hexanes/ethyl acetate, 50/1). M.p.: 111-113 ${ }^{\circ} \mathrm{C}$. $96 \%$ ee was determined by chiral HPLC (Chiralpak $\mathrm{AD}-\mathrm{H}, n$-hexane $/ \mathrm{i}-\mathrm{PrOH}=80 / 20,0.8 \mathrm{~mL} / \mathrm{min}, 254 \mathrm{~nm}, 40$ $\left.{ }^{\circ} \mathrm{C}\right): \mathrm{t}_{\mathrm{R}}($ major $)=26.8 \mathrm{~min}, \mathrm{t}_{\mathrm{R}}($ minor $)=10.0 \mathrm{~min} .[\alpha]_{\mathrm{D}}{ }^{23}=-67.1\left(c 0.52, \mathrm{CH}_{2} \mathrm{Cl}_{2}\right) .{ }^{1} \mathrm{H} \mathrm{NMR}(400 \mathrm{MHz}$, DMSO-d $\left.{ }^{6}\right): \delta$ 7.99-7.93 (m, 4H), 7.72-7.70 (m, 4H), 7.57-7.47 (m, 3H), $6.48(\mathrm{t}, J=2.9 \mathrm{~Hz}, 1 \mathrm{H})$, $5.55(\mathrm{~d}, J=3.3 \mathrm{~Hz}, 1 \mathrm{H}), 4.70(\mathrm{~d}, J=2.7 \mathrm{~Hz}, 1 \mathrm{H}), 3.68(\mathrm{~s}, 3 \mathrm{H}) ;{ }^{13} \mathrm{C}$ NMR $(101 \mathrm{MHz}$, DMSO-d $)$ : $\delta$ 
169.4, 164.2, 148.4, 137.3, 133.4, 133.1, 131.6, 131.5, 129.3, 129.3, 128.6, 128.1, 127.1, 127.1, 126.8, 125.1, 124.7, 107.1, 103.6, 87.5, 51.6. HRMS calc. for $\mathrm{C}_{23} \mathrm{H}_{18} \mathrm{BrO}_{3}[\mathrm{M}+\mathrm{H}]^{+}:$443.0259, found: 443.0261 .

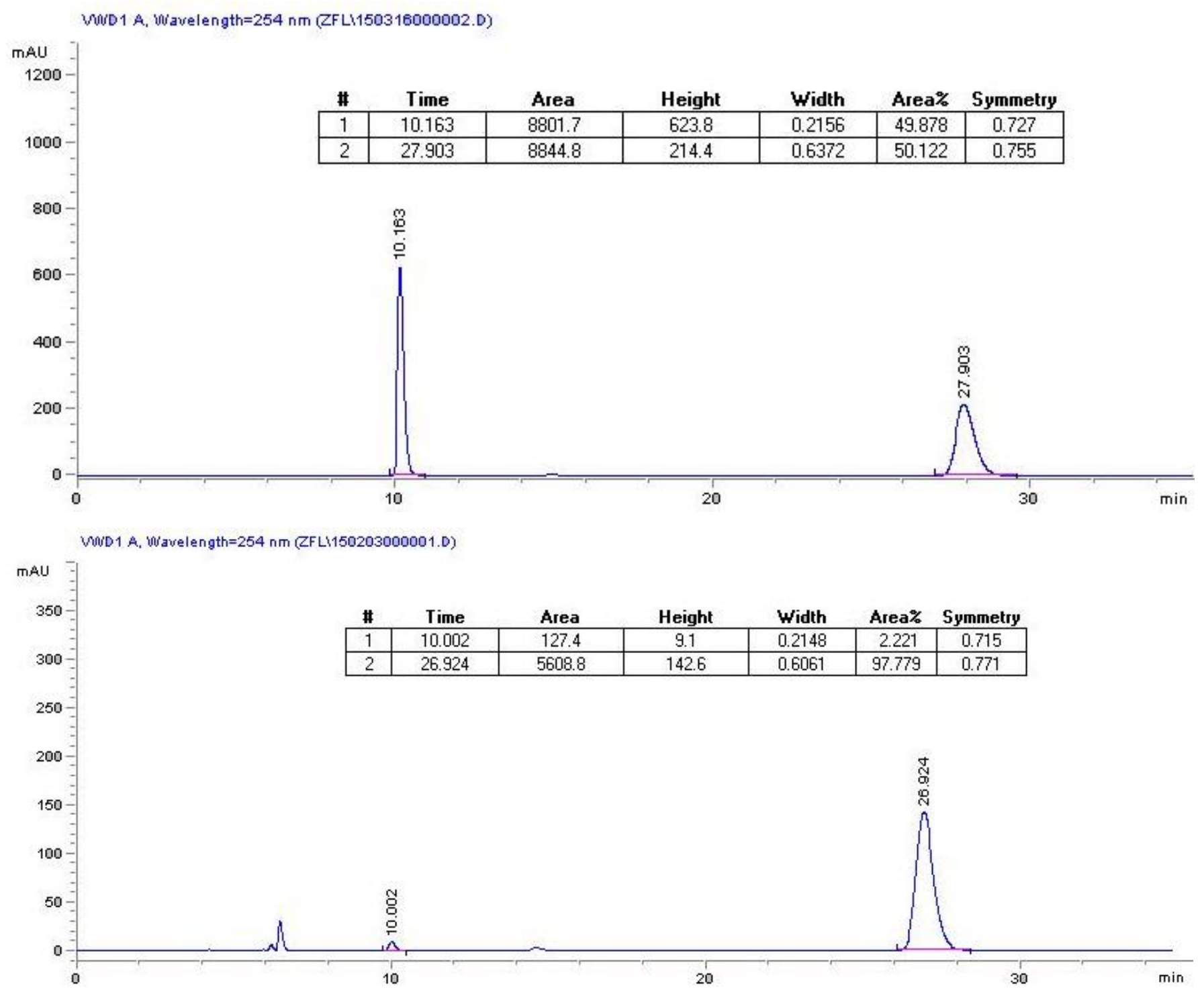

\section{Transformation of Cycloadduct 3aa}

\section{Hydrogenation of 3aa}

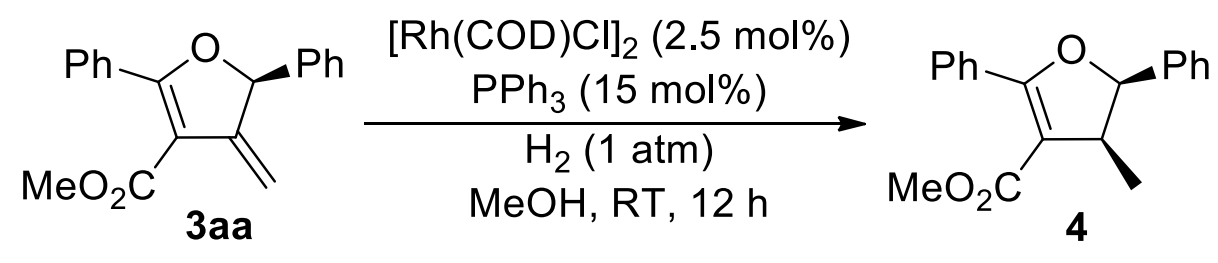


$[\mathrm{Rh}(\mathrm{COD}) \mathrm{Cl}]_{2}(2.5 \mathrm{mg}, 0.005 \mathrm{mmol})$ and $\mathrm{PPh}_{3}(7.9 \mathrm{mg}, 0.03 \mathrm{mmol})$ were stirred in $1 \mathrm{~mL}$ of anhydrous methanol at room temperature under nitrogen atmosphere for 20 minutes. A solution of $3 \mathrm{aa}(58.4 \mathrm{mg}, 0.20 \mathrm{mmol}$ ) in $1 \mathrm{~mL}$ of anhydrous methanol was added. The hydrogenation was performed at room temperature under $1 \mathrm{~atm}$ of $\mathrm{H}_{2}$ pressure for $12 \mathrm{~h}$. After concentration of the reaction mixture under reduced pressure, the residue was purified by silica gel chromatography (hexanes/ ethyl acetate, $50 / 1)$ to afford 4 (47.6 mg, $81 \%$ yield) as a colorless oil. $98 \%$ ee was determined by chiral HPLC (Chiralcel OJ-H, $n$-hexane/i-PrOH $=50 / 50,0.8 \mathrm{~mL} / \mathrm{min}, 254 \mathrm{~nm}, 40$ $\left.{ }^{\circ} \mathrm{C}\right): \operatorname{tR}($ major $)=25.1 \mathrm{~min}, \mathrm{tR}_{\mathrm{R}}($ minor $)=15.1 \mathrm{~min} .[\alpha]_{\mathrm{D}}{ }^{23}=148.1\left(\mathrm{c} 0.60, \mathrm{CH}_{2} \mathrm{Cl}_{2}\right) .{ }^{1} \mathrm{H}$ NMR $(400$ $\left.\mathrm{MHz}, \mathrm{CDCl}_{3}\right): \delta$ 7.87-7.85 (m, 2H), 7.44-7.30 (m, 8H), $5.80(\mathrm{~d}, J=8.9 \mathrm{~Hz}, 1 \mathrm{H}), 3.69(\mathrm{~s}, 3 \mathrm{H})$, 3.55-3.49 (m, $1 \mathrm{H}), 0.81(\mathrm{~d}, J=7.0 \mathrm{~Hz}, 3 \mathrm{H}) ;{ }^{13} \mathrm{C} \mathrm{NMR}\left(101 \mathrm{MHz}, \mathrm{CDCl}_{3}\right): \delta 165.7,165.1,136.9$, 130.6, 130.1, 129.6, 128.3, 127.8, 127.7, 126.3, 109.4, 87.1, 51.0, 42.8, 15.9. HRMS calc. for $\mathrm{C}_{19} \mathrm{H}_{19} \mathrm{O}_{3}[\mathrm{M}+\mathrm{H}]^{+}: 295.1334$, found: 295.1337 .
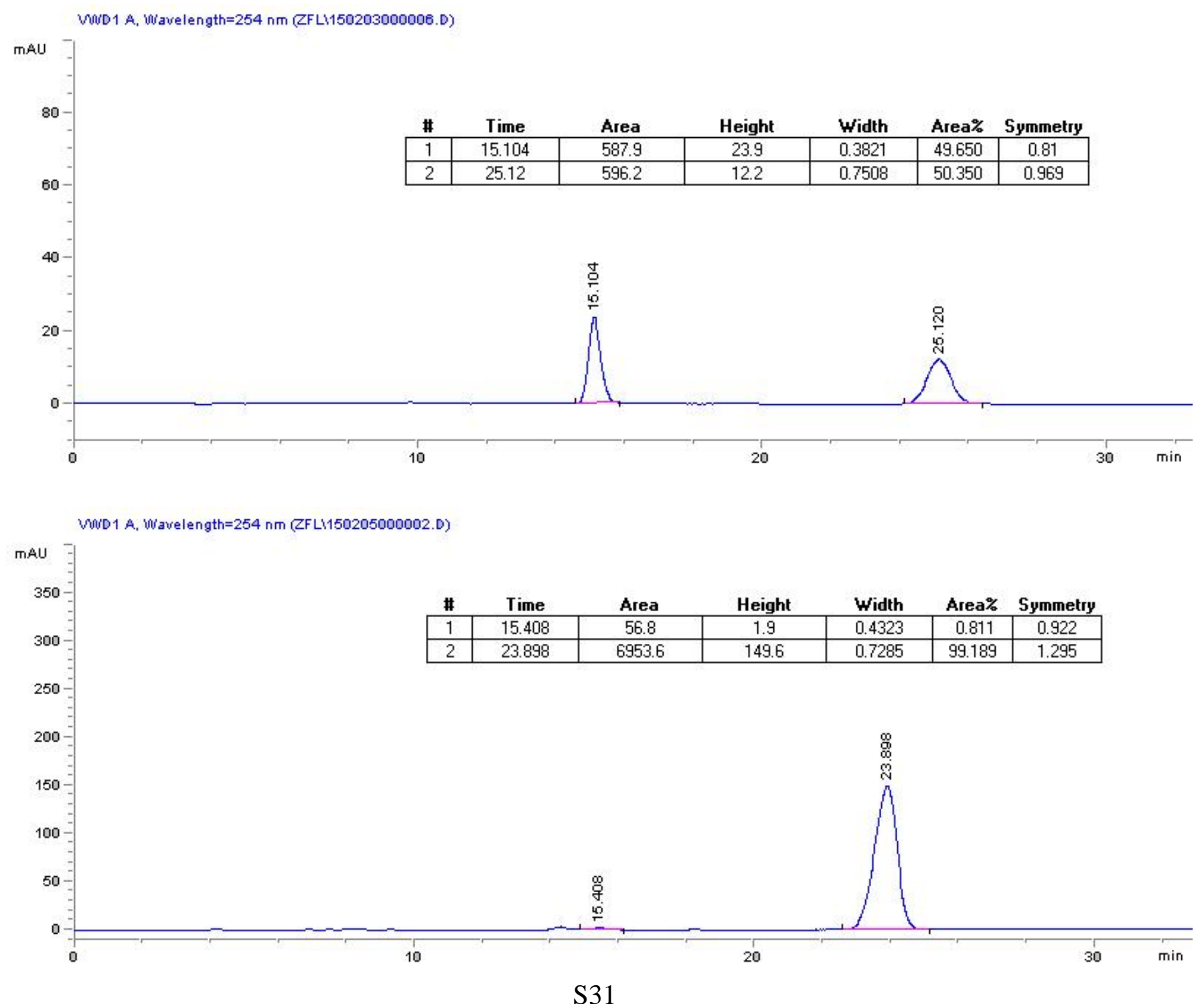

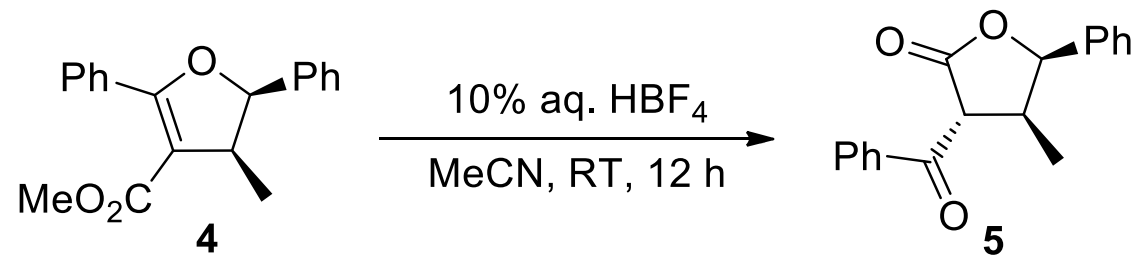

To a solution of $4(59.0 \mathrm{mg}, 0.20 \mathrm{mmol})$ in $1 \mathrm{~mL}$ of acetonitrile was added $0.2 \mathrm{~mL}$ of aqueous $\mathrm{HBF}_{4}(10 \%)$. The mixture was stirred at room temperature for $12 \mathrm{~h}$. The reaction was diluted by 8 $\mathrm{mL}$ of water and extracted with diethyl ether $(10 \mathrm{~mL} \times 3)$. The combined extracts were washed with water, dried over anhydrous $\mathrm{Na}_{2} \mathrm{SO}_{4}$, and concentrated under vaccum, the residue was then purified by silica gel chromatography (hexanes/ ethyl acetate, 20/1) to afford a colorless oil 5 (45.9 mg, 82\% yield). ${ }^{1} \mathrm{H}$ NMR (400 MHz, $\left.\mathrm{CDCl}_{3}\right): \delta 8.11-8.09(\mathrm{~m}, 2 \mathrm{H}), 7.66-7.26(\mathrm{~m}, 8 \mathrm{H}), 5.89(\mathrm{~d}$, $J=6.6 \mathrm{~Hz}, 1 \mathrm{H}), 4.37(\mathrm{~d}, J=4.6 \mathrm{~Hz}, 1 \mathrm{H}), 3.39(\mathrm{qd}, J=7.1,4.7 \mathrm{~Hz}, 1 \mathrm{H}), 0.81(\mathrm{~d}, J=7.2 \mathrm{~Hz}, 3 \mathrm{H})$; ${ }^{13} \mathrm{C}$ NMR $\left(101 \mathrm{MHz}, \mathrm{CDCl}_{3}\right): \delta 192.8,172.2,135.8,135.4,134.2,129.4,128.9,128.6,128.3$, 125.5, 83.5, 57.3, 38.9, 15.1. HRMS calc. for $\mathrm{C}_{18} \mathrm{H}_{17} \mathrm{O} 33[\mathrm{M}+\mathrm{H}]^{+}: 281.1178$, found: 281.1177 .

\section{References}

1. EidJr, C. N.; Konopelski, J. P. Tetrahedron 1991, 47, 975.

2. (a) Fang, P.; Hou, X.-L. Org. Lett. 2009, 11, 4612. (b) Bhanuchandra, M.; Kuram, M. R.; Sahoo, A. K. J. Org. Chem. 2013, 78, 11824.

3. (a) Hayashi, T.; Mise, T.; Fukushima, M.; Kagotani, M.; Nagashima, N.; Hamada, Y.; Matsumoto, A.; Kawakami, S.; Konishi, M.; Yamamoto, K.; Kumada, M. Bull. Chem. Soc. Jpn. 1980, 53, 1138. (b) Boaz, N. W.; Debenham, S. D.; Mackenzie, E. B.; Large, S. E. Org. Lett. 2002, 4, 2421. (c) Hu, X.-P.; Bai, C.-M.; Dai, H.-C.; Chen, H.-L.; Zheng, Z. J. Mol. Catal. A. Chem. 2008, 73, 6022. 


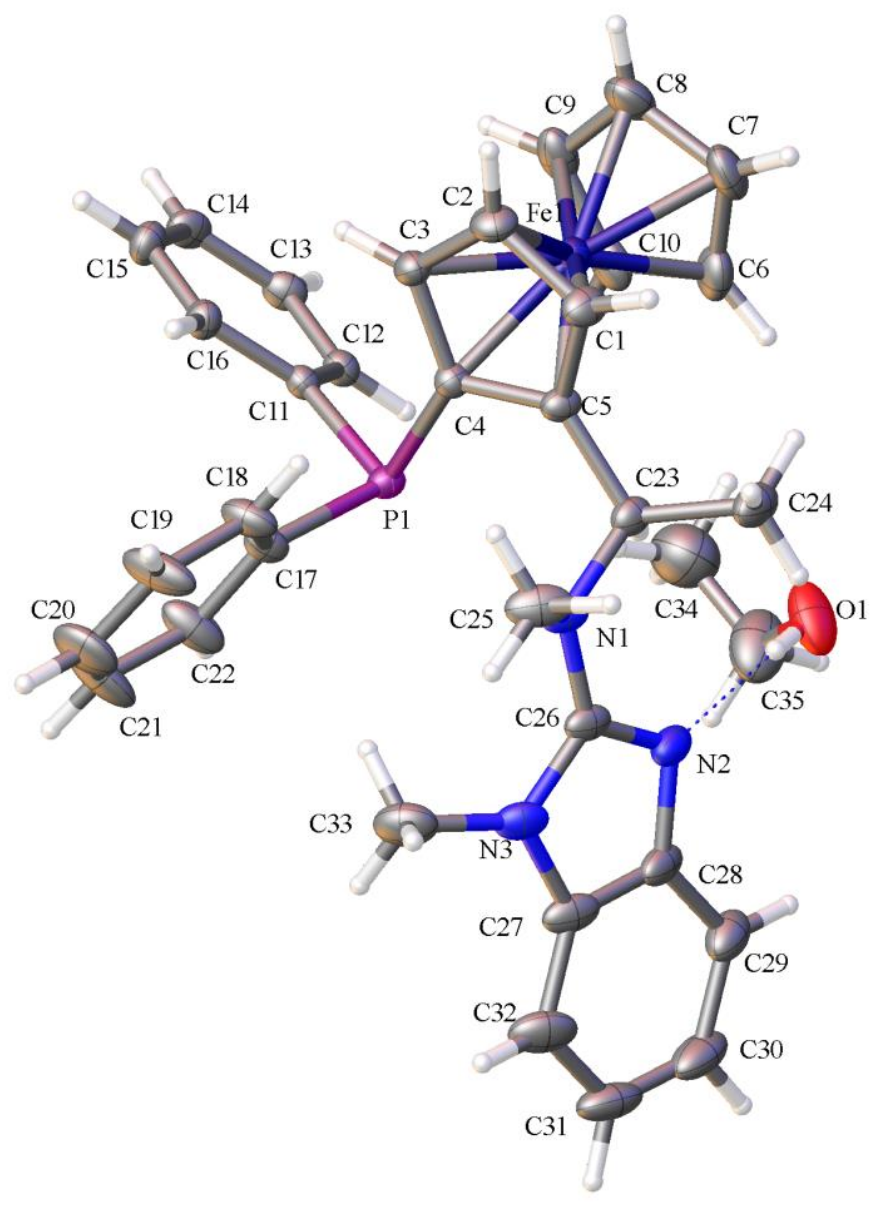

Table 1. Crystal data and structure refinement for sa3830.

Identification code

Empirical formula

Formula weight

Temperature

Wavelength

Crystal system

Space group

Unit cell dimensions

Volume

$\mathrm{Z}$

Density (calculated)

Absorption coefficient sa3830

C35 H38 Fe N3 O P

603.50

$173.1500 \mathrm{~K}$

$0.71073 \AA$

Orthorhombic

P 212121

$a=10.678(2) \AA \quad \alpha=90^{\circ}$.

$\mathrm{b}=10.701(2) \AA \quad \beta=90^{\circ}$.

$\mathrm{c}=27.346(6) \AA \quad \gamma=90^{\circ}$.

3124.8(11) $\AA^{3}$

4

$1.283 \mathrm{Mg} / \mathrm{m}^{3}$

$0.565 \mathrm{~mm}^{-1}$ 
F(000)

Crystal size

Theta range for data collection

Index ranges

Reflections collected

Independent reflections

Completeness to theta $=26.000^{\circ}$

Absorption correction

Max. and min. transmission

Refinement method

Data / restraints / parameters

Goodness-of-fit on $\mathrm{F}^{2}$

Final $\mathrm{R}$ indices [I $>2 \operatorname{sigma}(\mathrm{I})]$

$\mathrm{R}$ indices (all data)

Absolute structure parameter

Extinction coefficient

Largest diff. peak and hole
1272

$0.521 \times 0.315 \times 0.115 \mathrm{~mm}^{3}$

1.489 to $27.478^{\circ}$.

$-13<=\mathrm{h}<=13,-13<=\mathrm{k}<=13,-35<=\mathrm{l}<=35$

21674

$6729[\mathrm{R}(\mathrm{int})=0.0453]$

$99.5 \%$

Semi-empirical from equivalents

1.0000 and 0.8537

Full-matrix least-squares on $\mathrm{F}^{2}$

6729 / 0 / 375

1.095

$\mathrm{R} 1=0.0483, \mathrm{wR} 2=0.0965$

$\mathrm{R} 1=0.0524, \mathrm{wR} 2=0.0985$

$0.044(10)$

$\mathrm{n} / \mathrm{a}$

0.421 and -0.311 e. $\AA^{-3}$

\section{Crystal Data and Structure Refinement for $(S)-3$ ej}

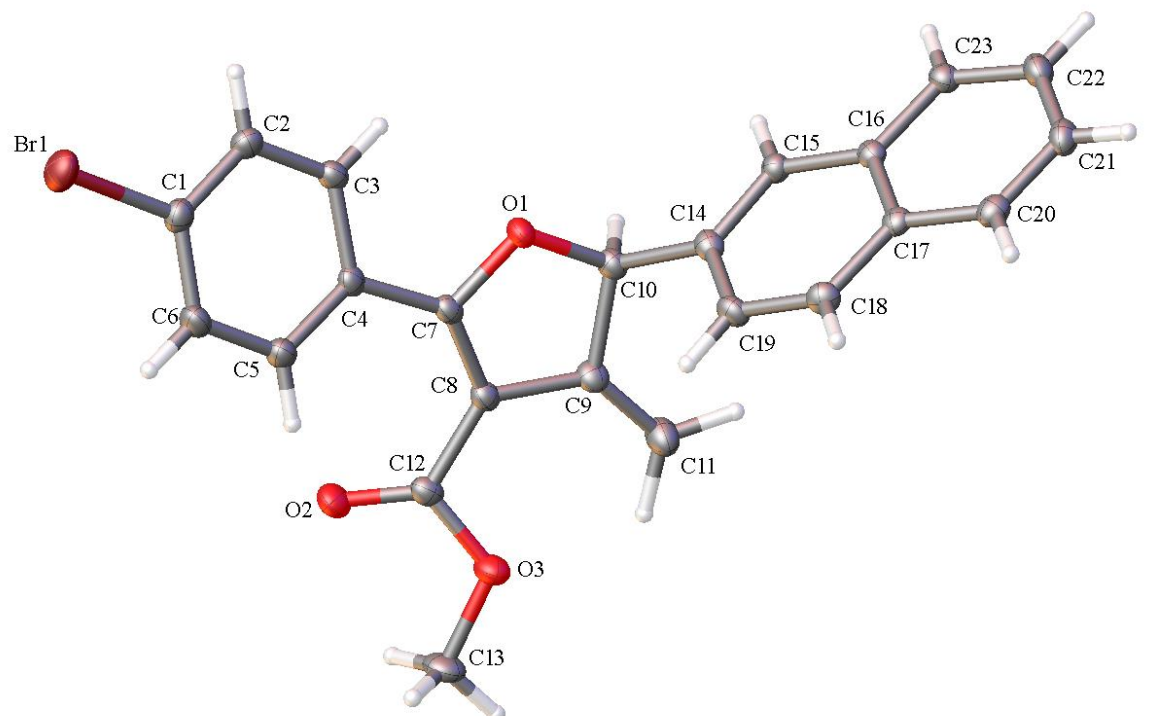

Table 1. Crystal data and structure refinement for sa3855.

Identification code

Empirical formula

Formula weight

Temperature sa3855

421.27

$173.1500 \mathrm{~K}$ 
Wavelength

Crystal system

Space group

Unit cell dimensions

Volume

$\mathrm{Z}$

Density (calculated)

Absorption coefficient

$\mathrm{F}(000)$

Crystal size

Theta range for data collection

Index ranges

Reflections collected

Independent reflections

Completeness to theta $=26.000^{\circ}$

Absorption correction

Max. and min. transmission

Refinement method

Data / restraints / parameters

Goodness-of-fit on $\mathrm{F}^{2}$

Final $\mathrm{R}$ indices [I $>2 \operatorname{sigma}(\mathrm{I})]$

$\mathrm{R}$ indices (all data)

Absolute structure parameter

Extinction coefficient

Largest diff. peak and hole
$0.71073 \AA$

Monoclinic

P 1211

$$
\begin{aligned}
& a=5.4774(11) \AA \quad \alpha=90^{\circ} \text {. } \\
& \mathrm{b}=14.656(3) \AA \quad \beta=103.09(3)^{\circ} \text {. } \\
& \mathrm{c}=11.788(2) \AA \quad \gamma=90^{\circ} \text {. }
\end{aligned}
$$

921.8(3) $\AA^{3}$

2

\section{$1.518 \mathrm{Mg} / \mathrm{m}^{3}$}

$2.251 \mathrm{~mm}^{-1}$

428

$0.35 \times 0.29 \times 0.2 \mathrm{~mm}^{3}$

3.298 to $27.483^{\circ}$.

$-7<=\mathrm{h}<=7,-19<=\mathrm{k}<=18,-15<=\mathrm{l}<=15$

10187

$4129[\mathrm{R}(\mathrm{int})=0.0348]$

$99.6 \%$

Semi-empirical from equivalents

1.0000 and 0.6665

Full-matrix least-squares on $\mathrm{F}^{2}$

4129 / 1 / 253

1.087

$\mathrm{R} 1=0.0336, \mathrm{wR} 2=0.0781$

$\mathrm{R} 1=0.0355, \mathrm{wR} 2=0.0793$

$0.008(6)$

$\mathrm{n} / \mathrm{a}$

0.257 and -0.450 e. $\AA^{-3}$ 


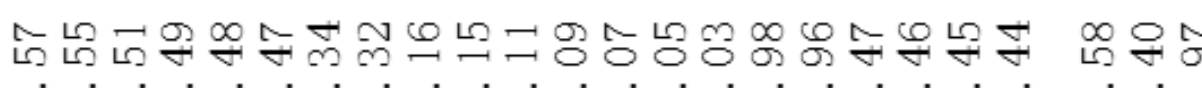

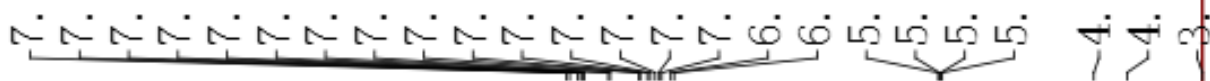
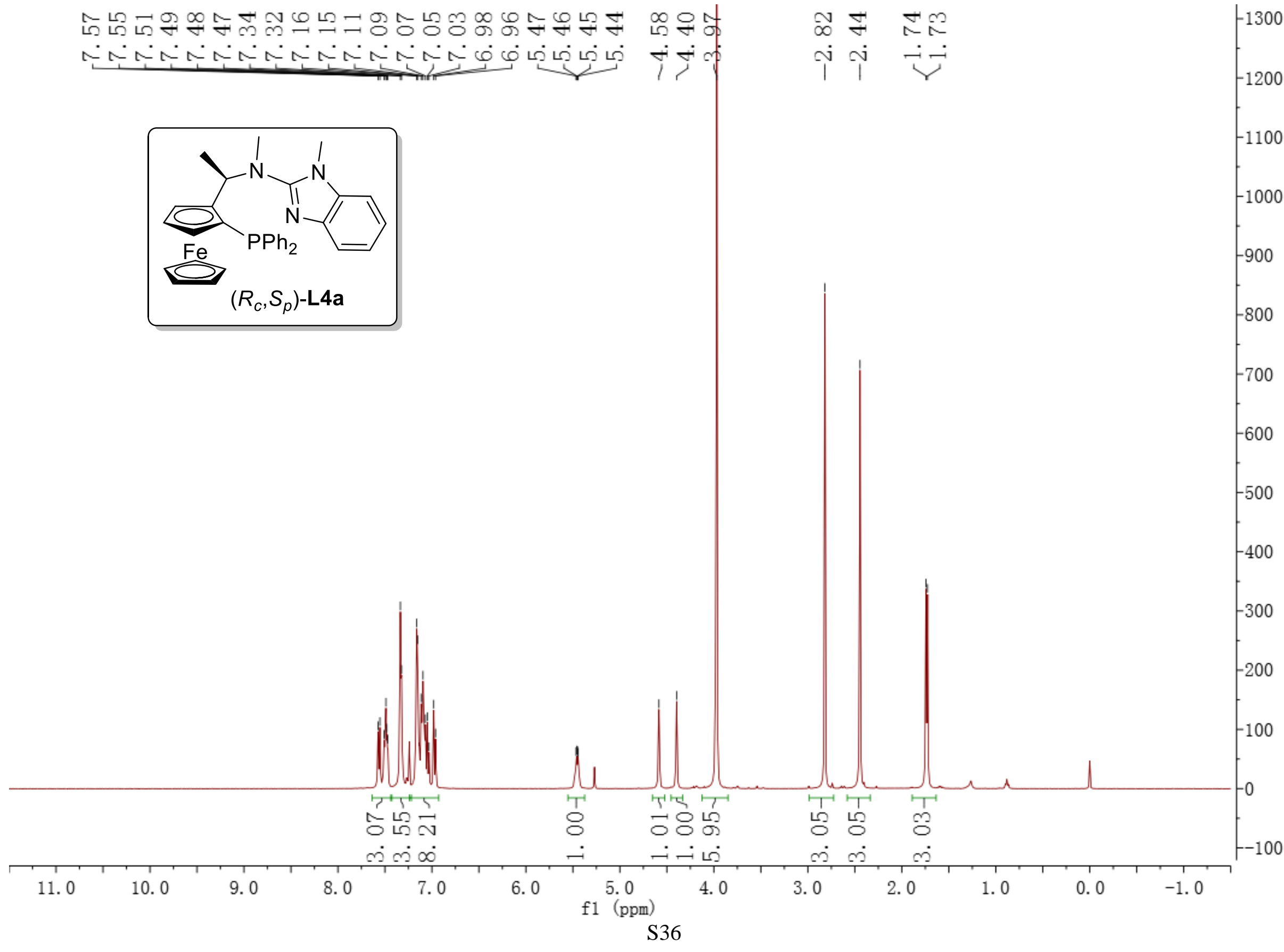
드르요

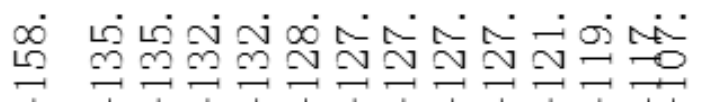

단ㄷㄴ

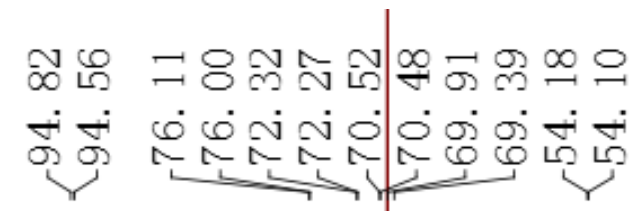

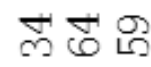

용

क

रो
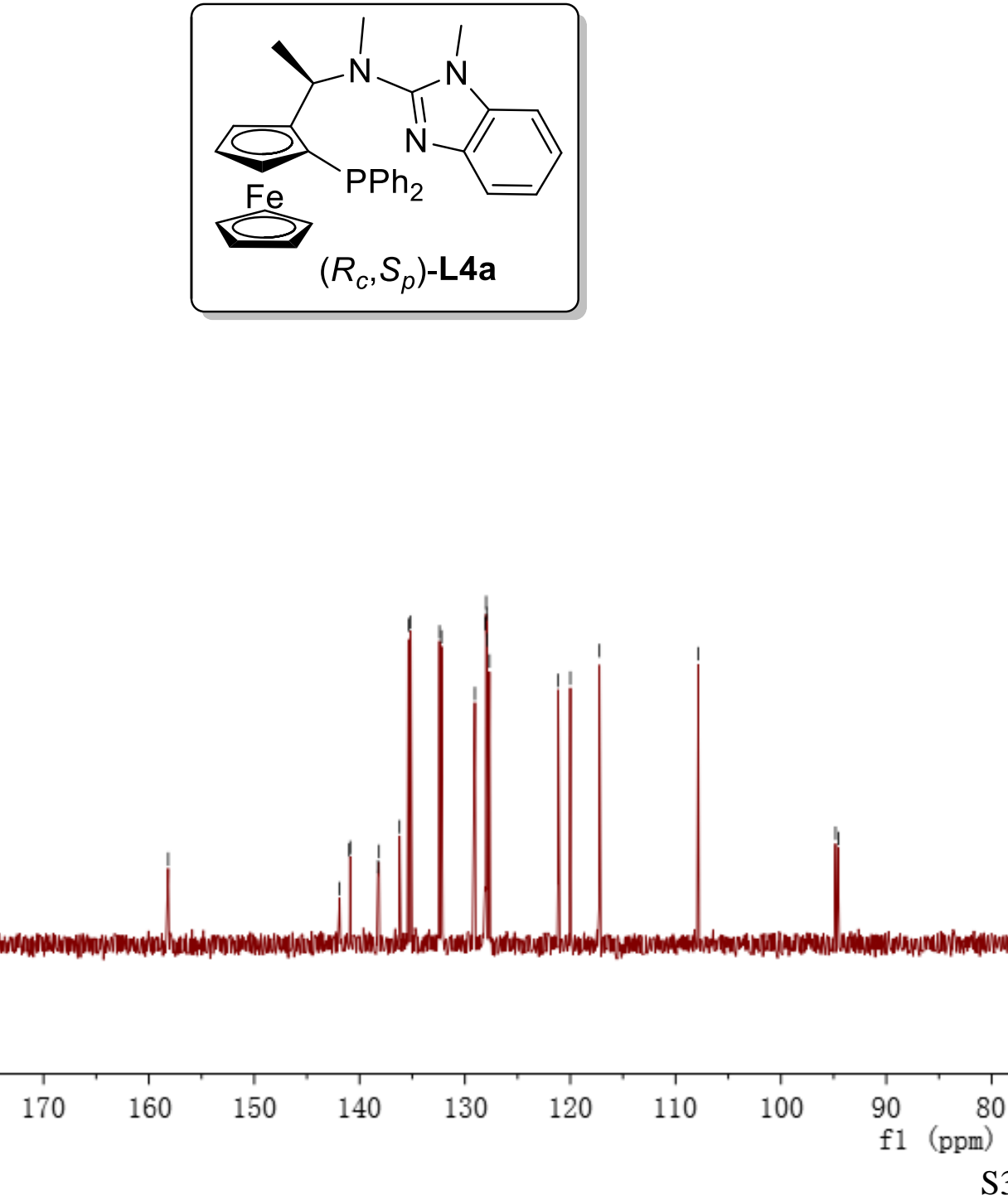


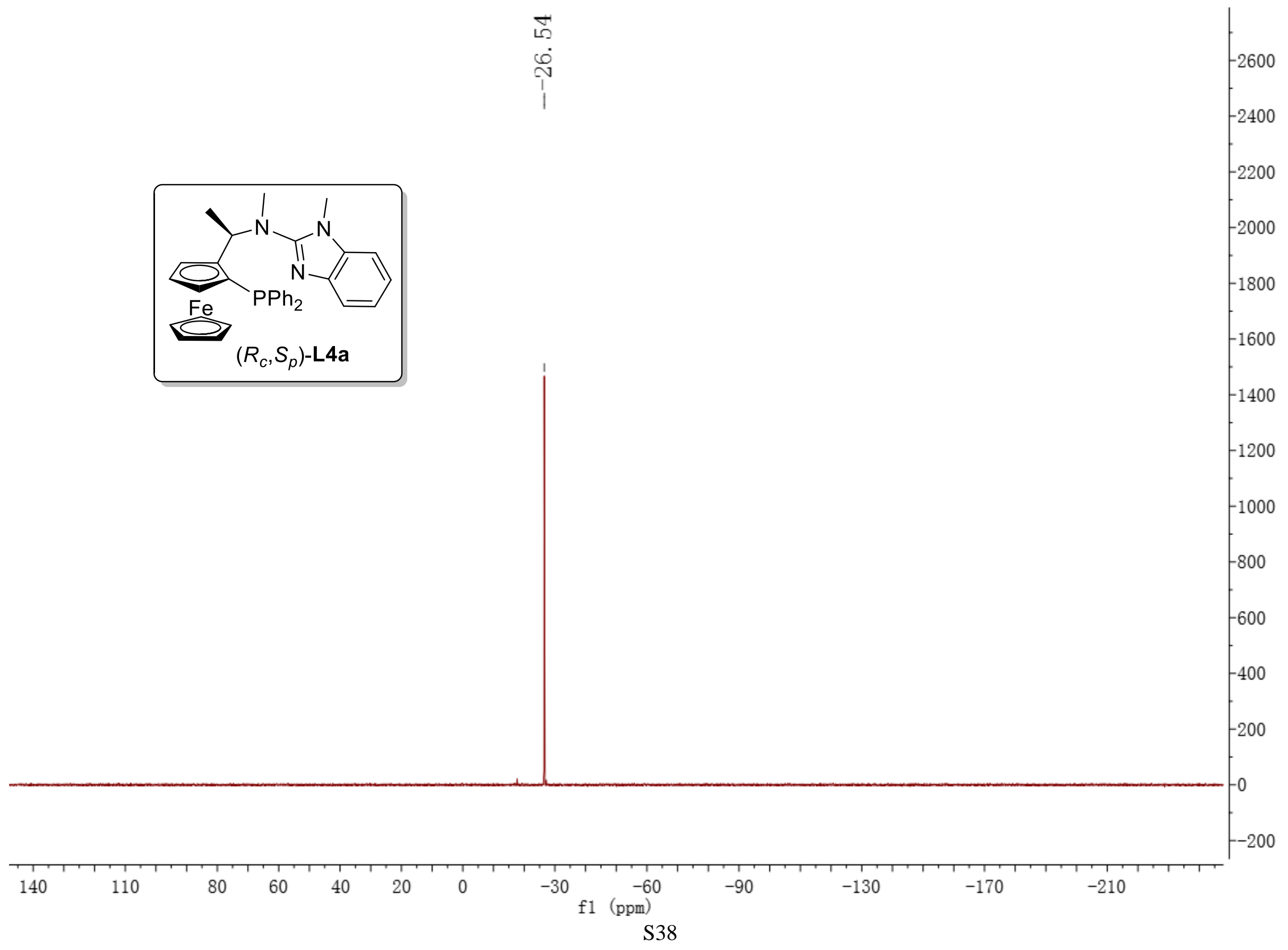




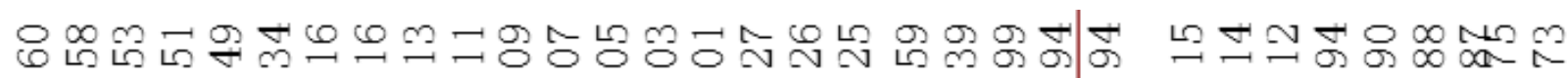

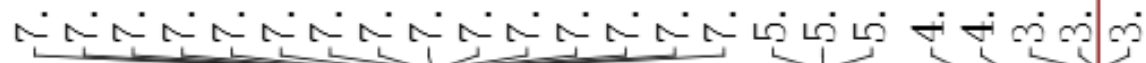
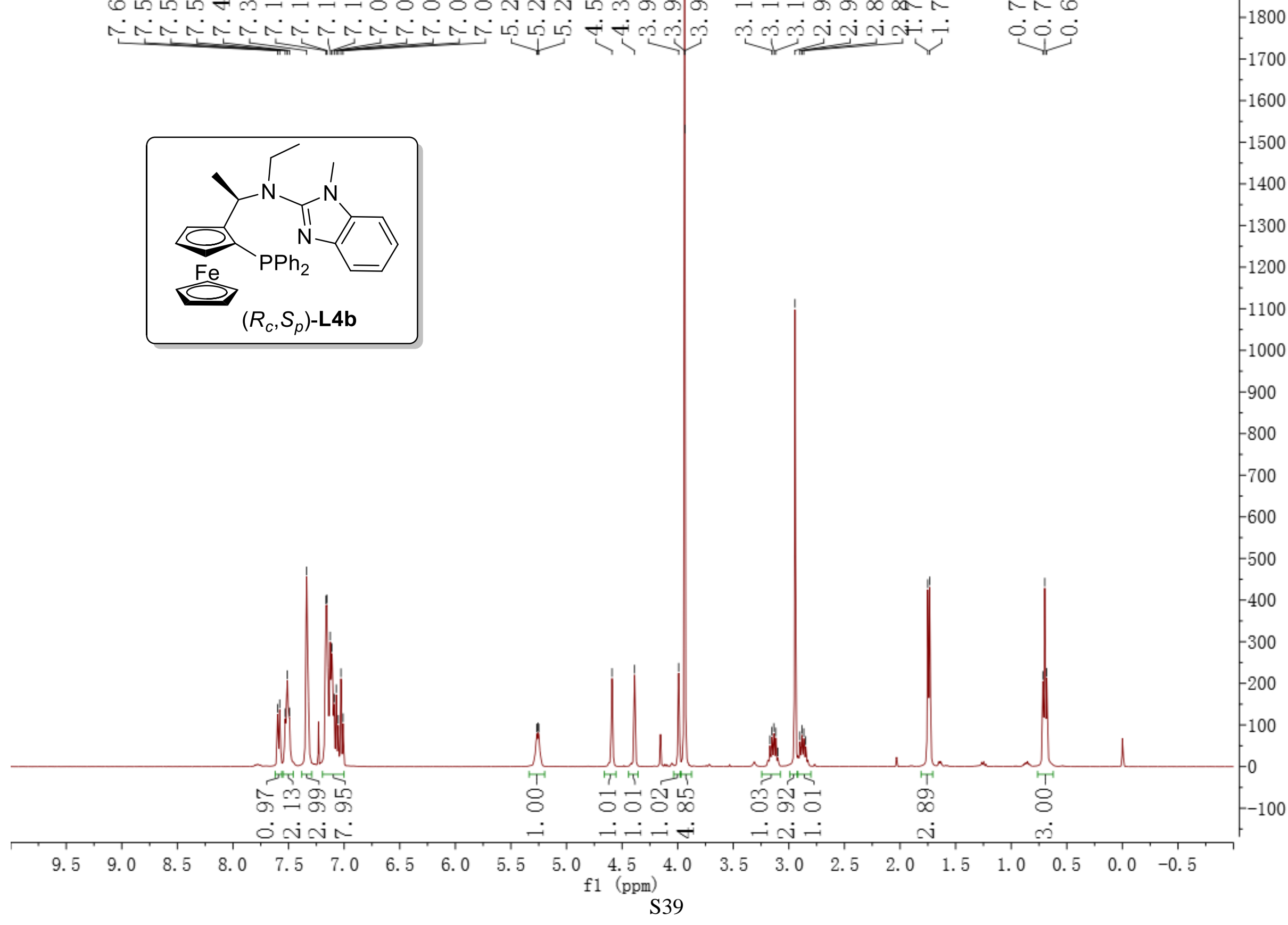

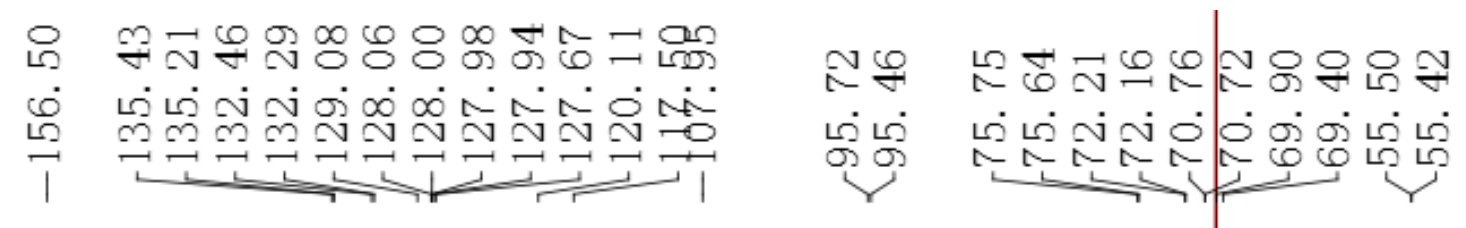

용
용

용

ம்
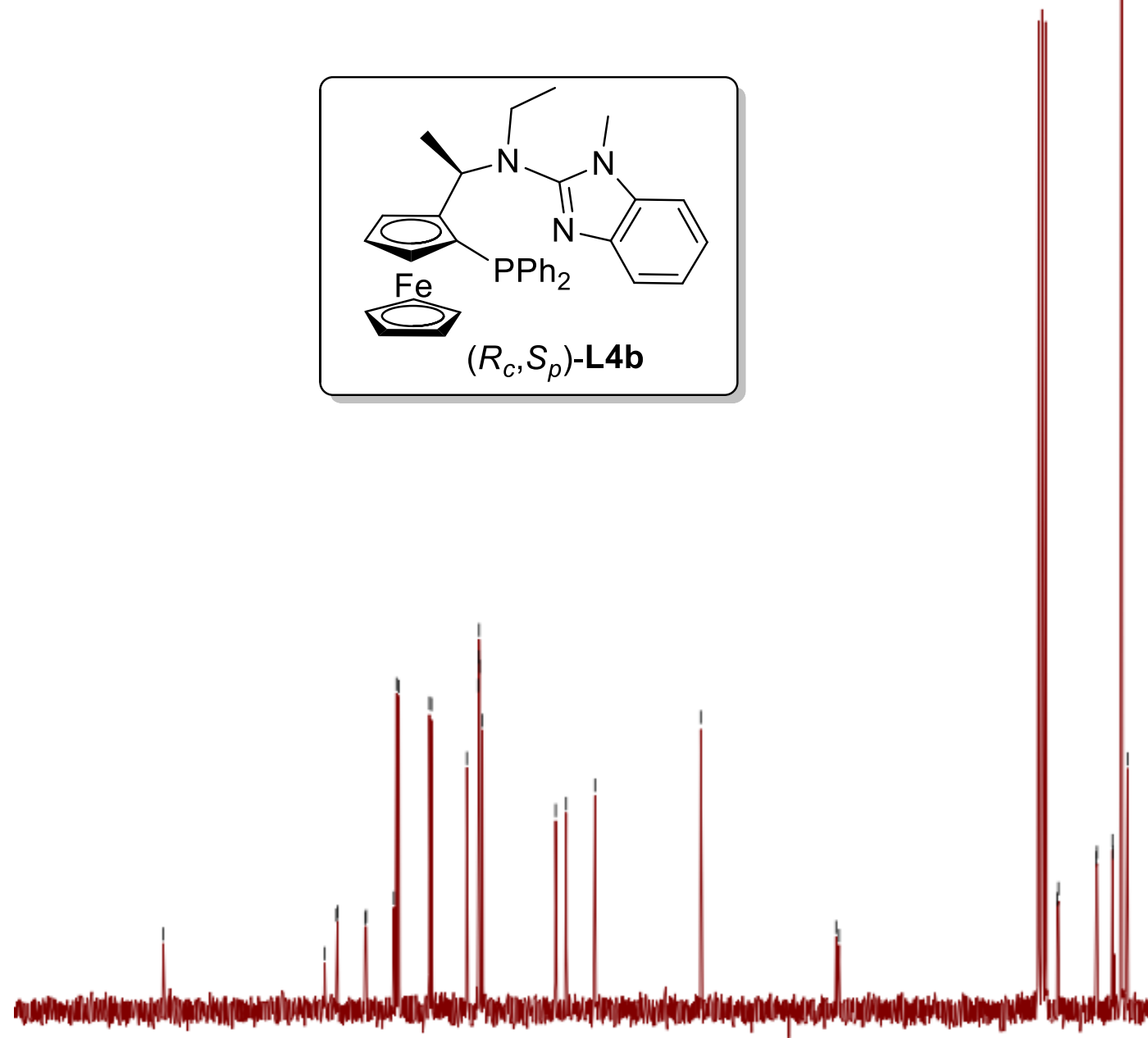


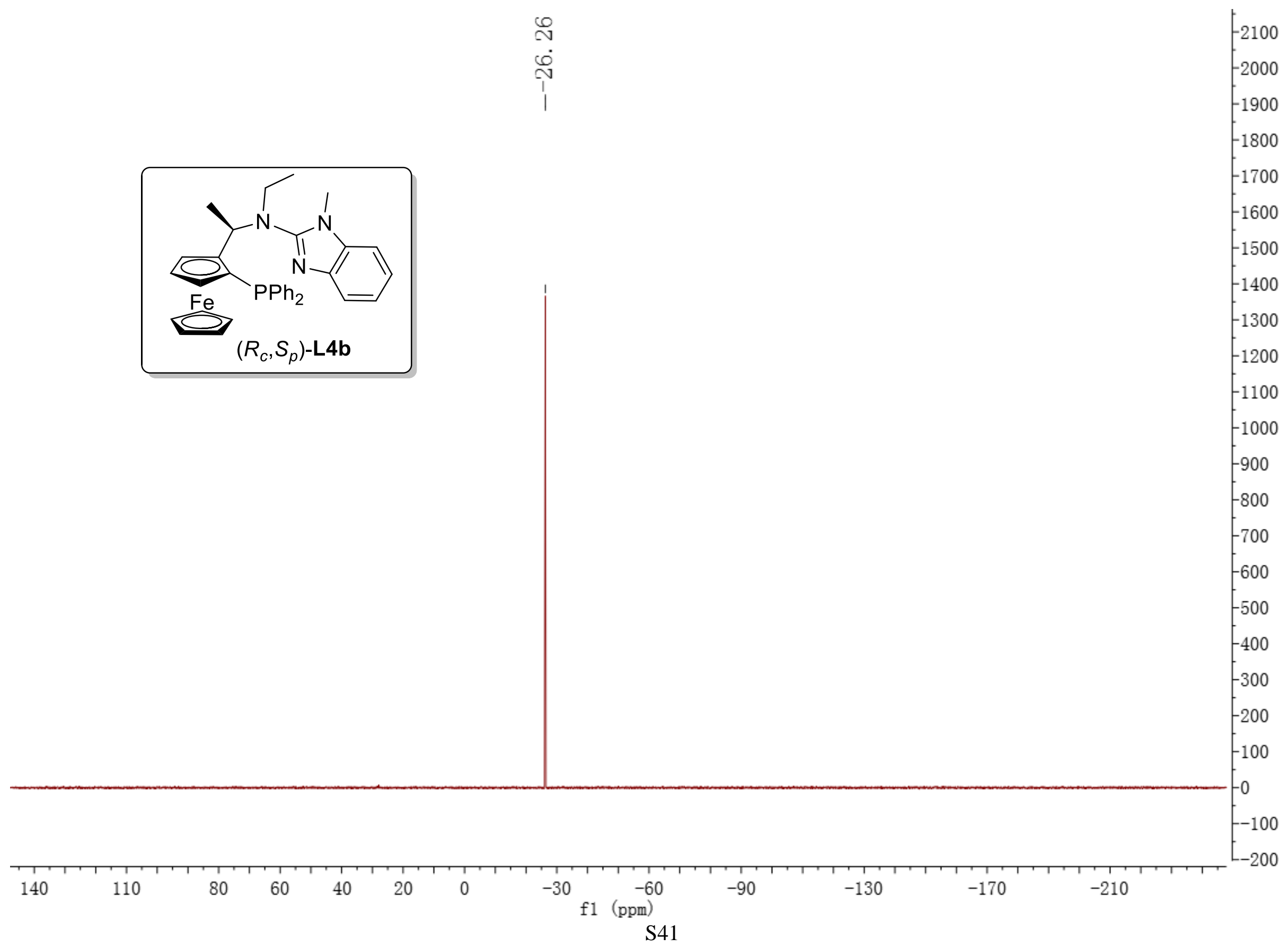




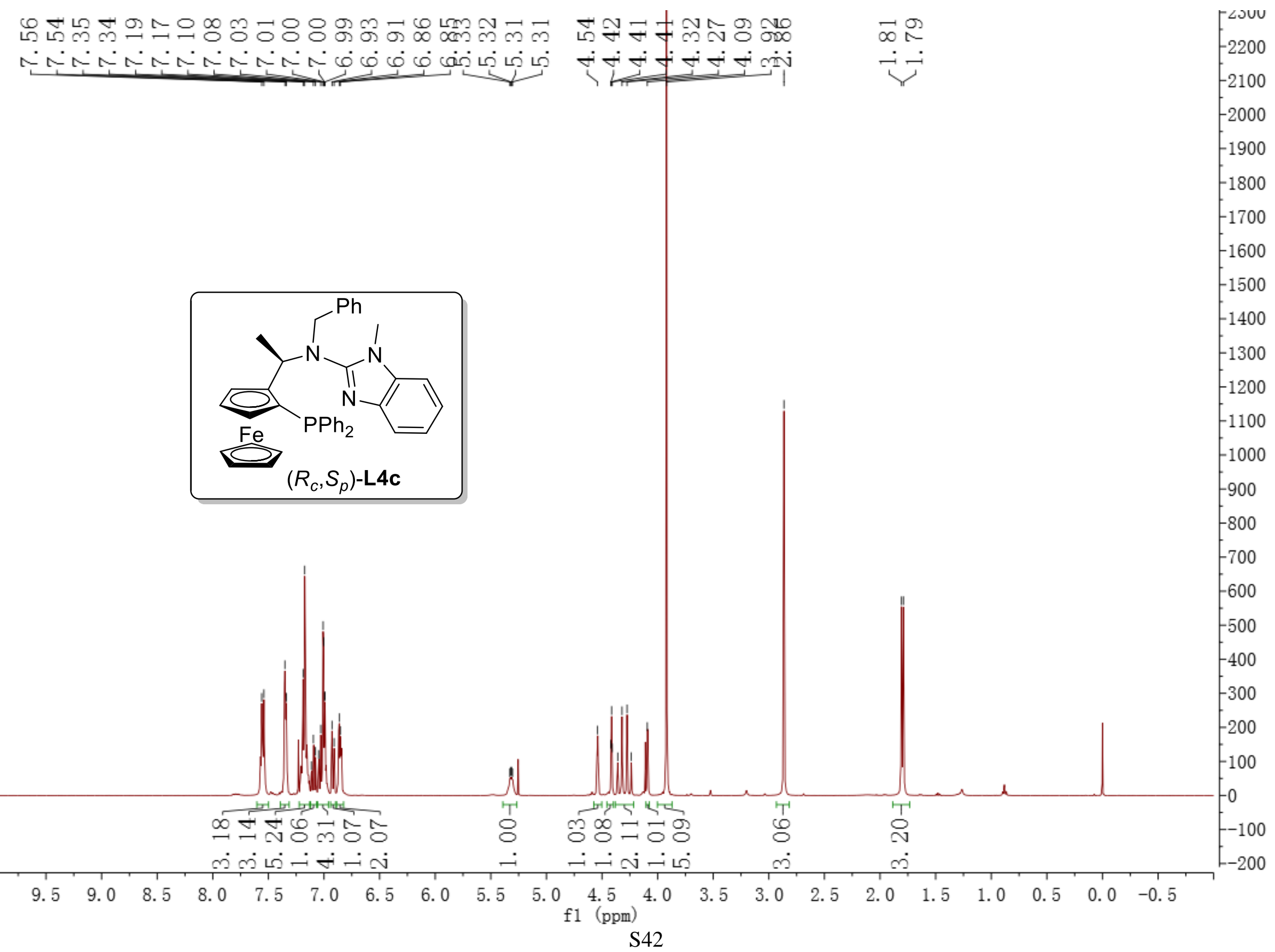




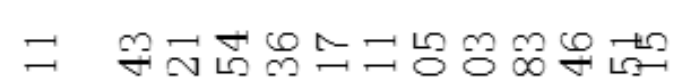

占

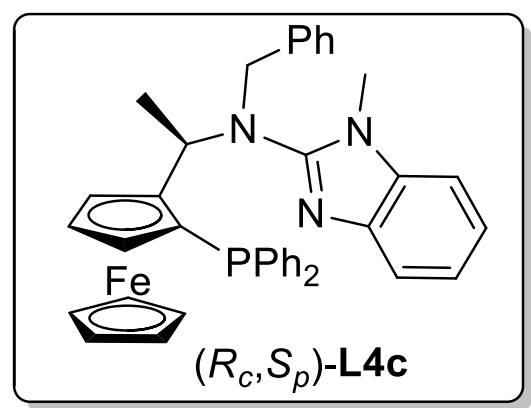

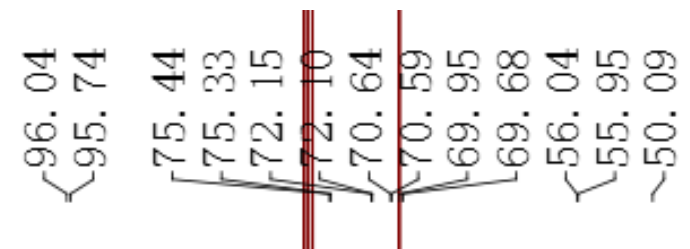

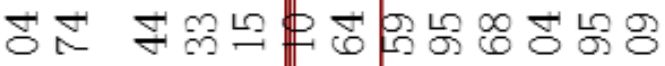

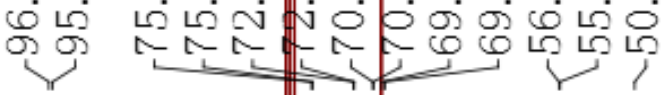

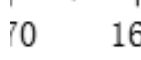

150

140

130

120

110

100

90

$\mathrm{f} 1$ (ppm) 


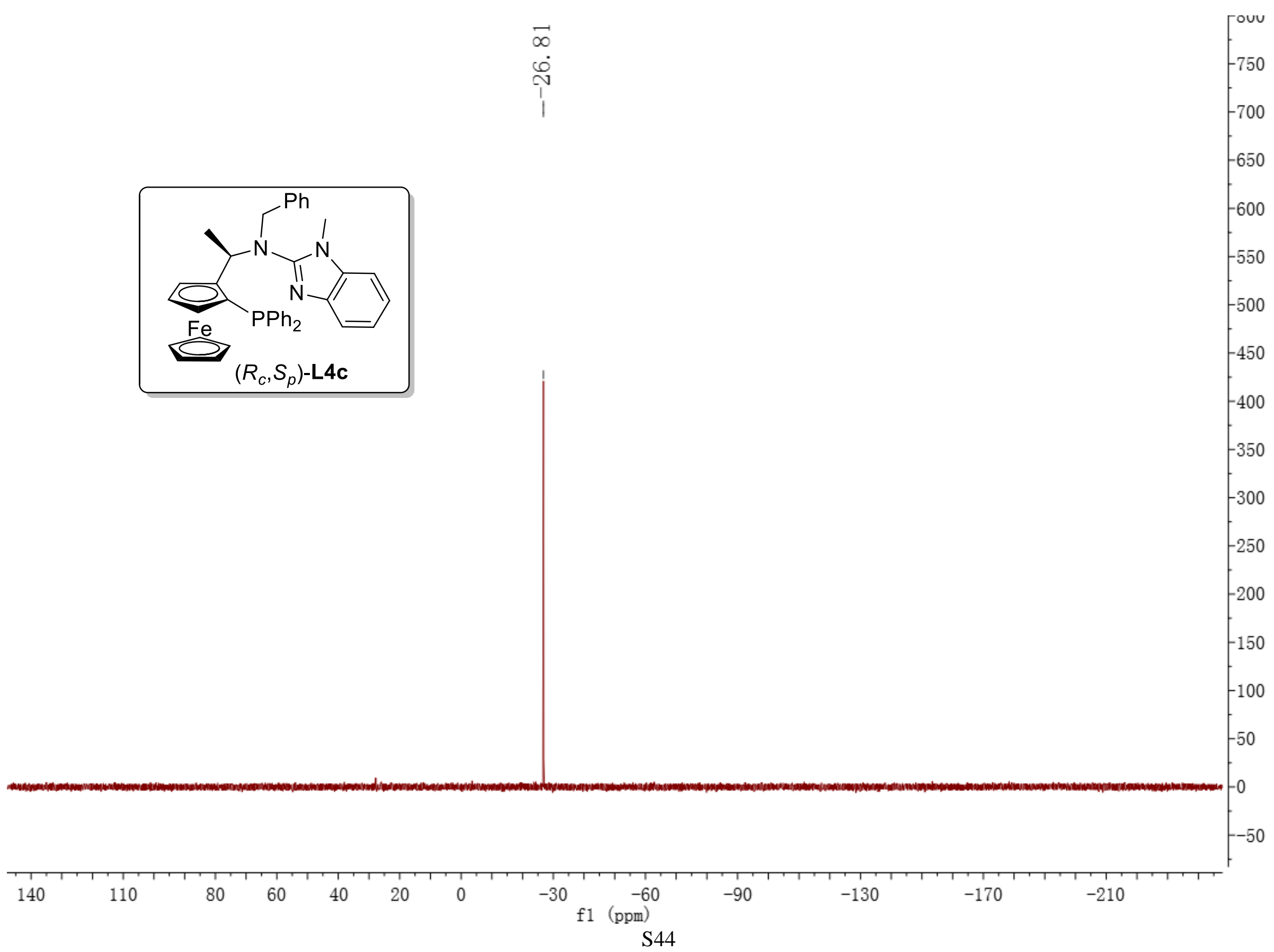




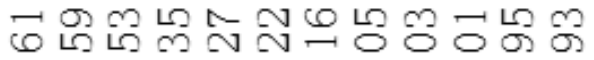

NNNNNNNNNNEO

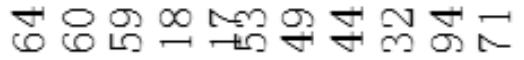

نَ

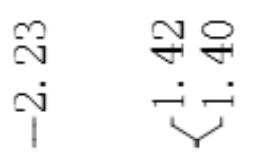

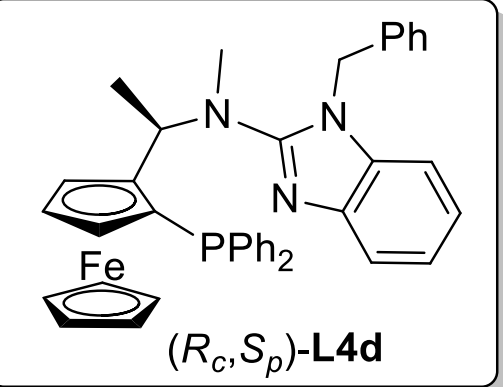

$\left(R_{c}, S_{p}\right)$-L4d

$\because \neg .7$ ம் - $-\dot{-}$ 


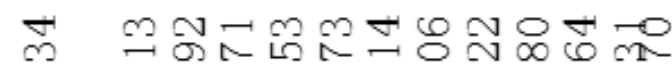

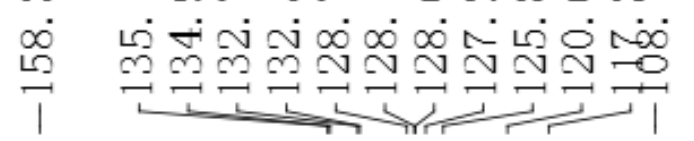

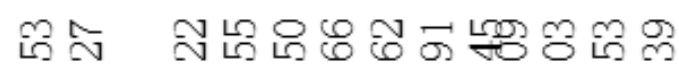

ন

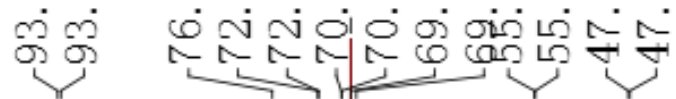

$\stackrel{\text { i่ }}{\text { i }}$

$-1100$
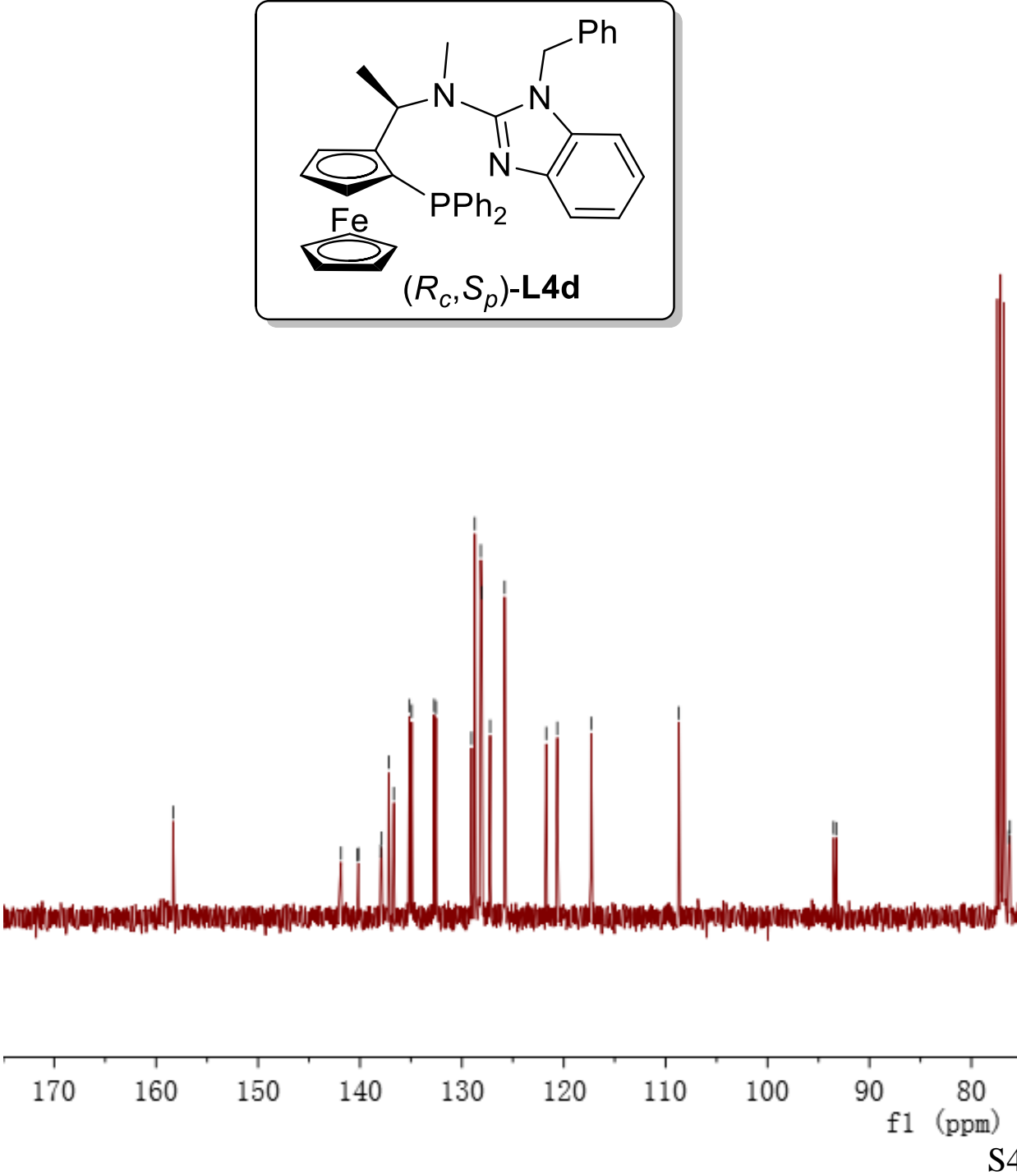


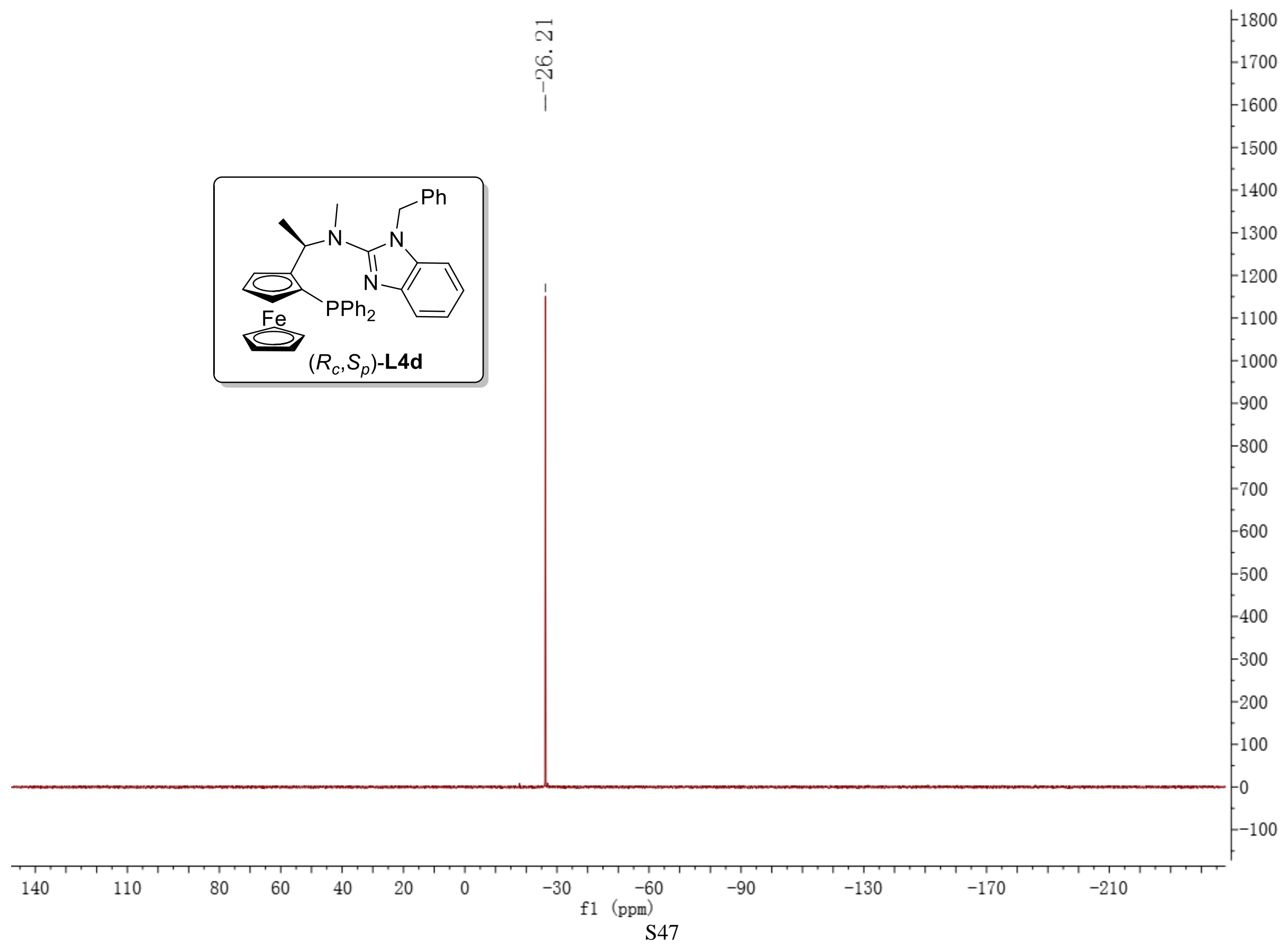




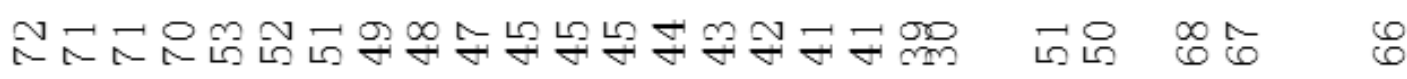

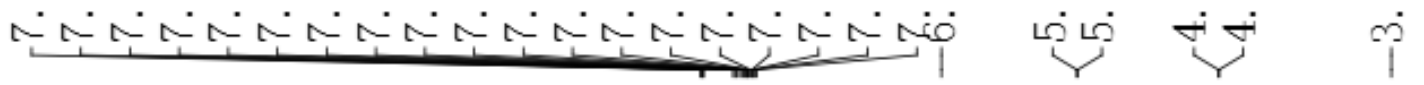

-23000
-22000
-21000
-20000
-19000
-18000
-17000
-16000
-15000
-14000
-13000
-12000
-11000
-10000
-9000
-8000
-7000
-6000
-5000
-4000
-3000
-2000
-1000
-0
-1000
-2000




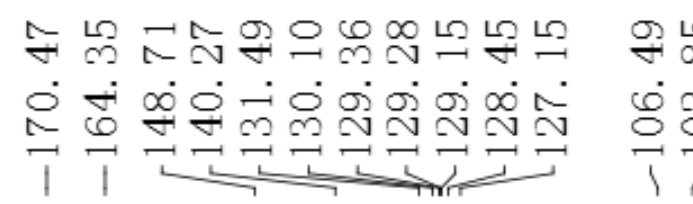

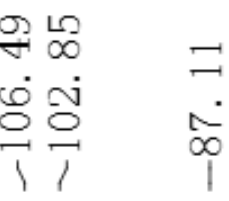

$\underset{1}{\approx} \quad \frac{\pi}{1}$

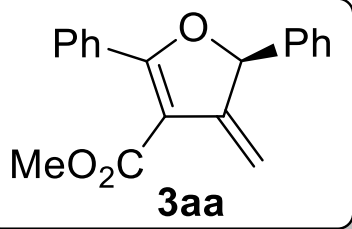



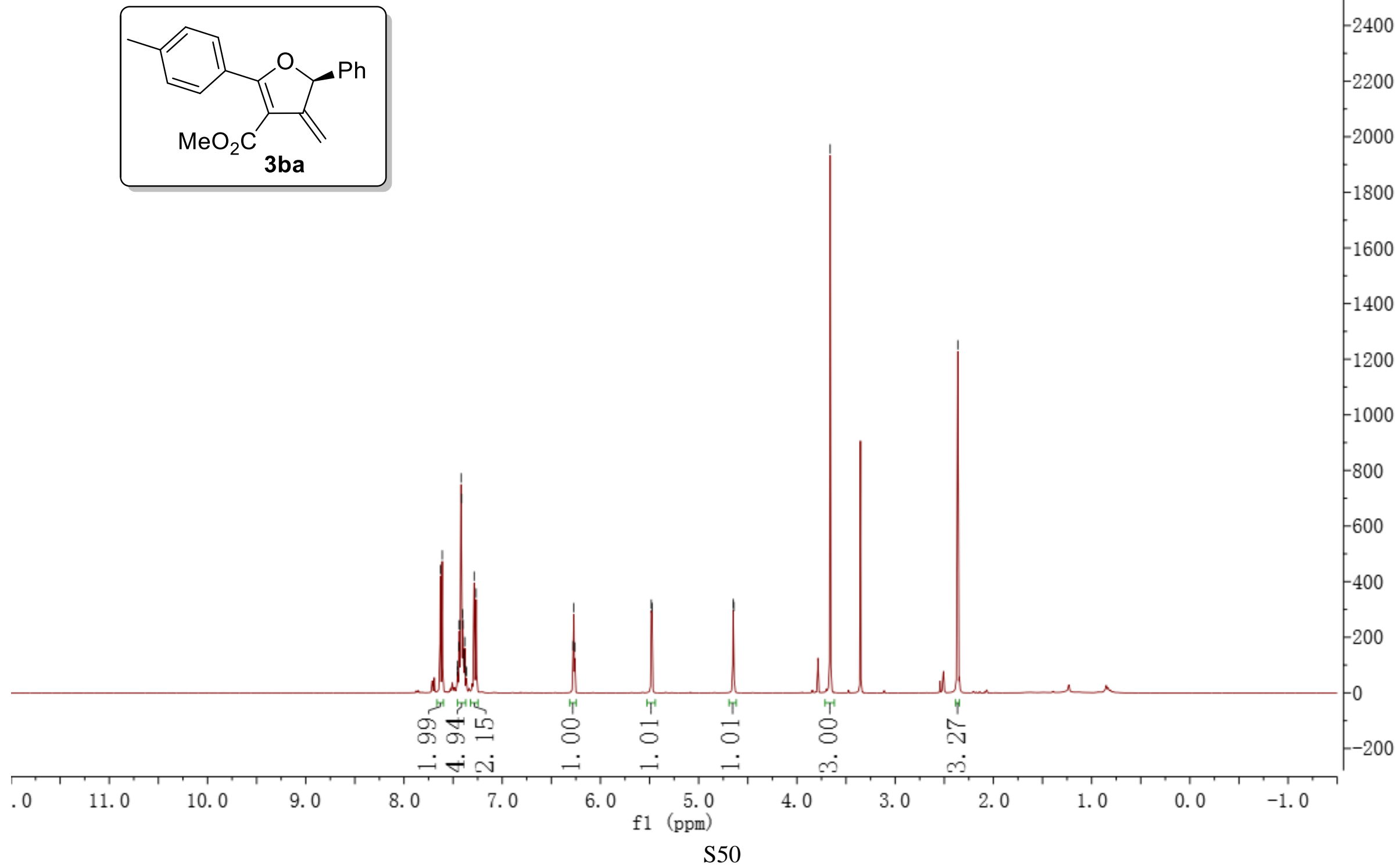


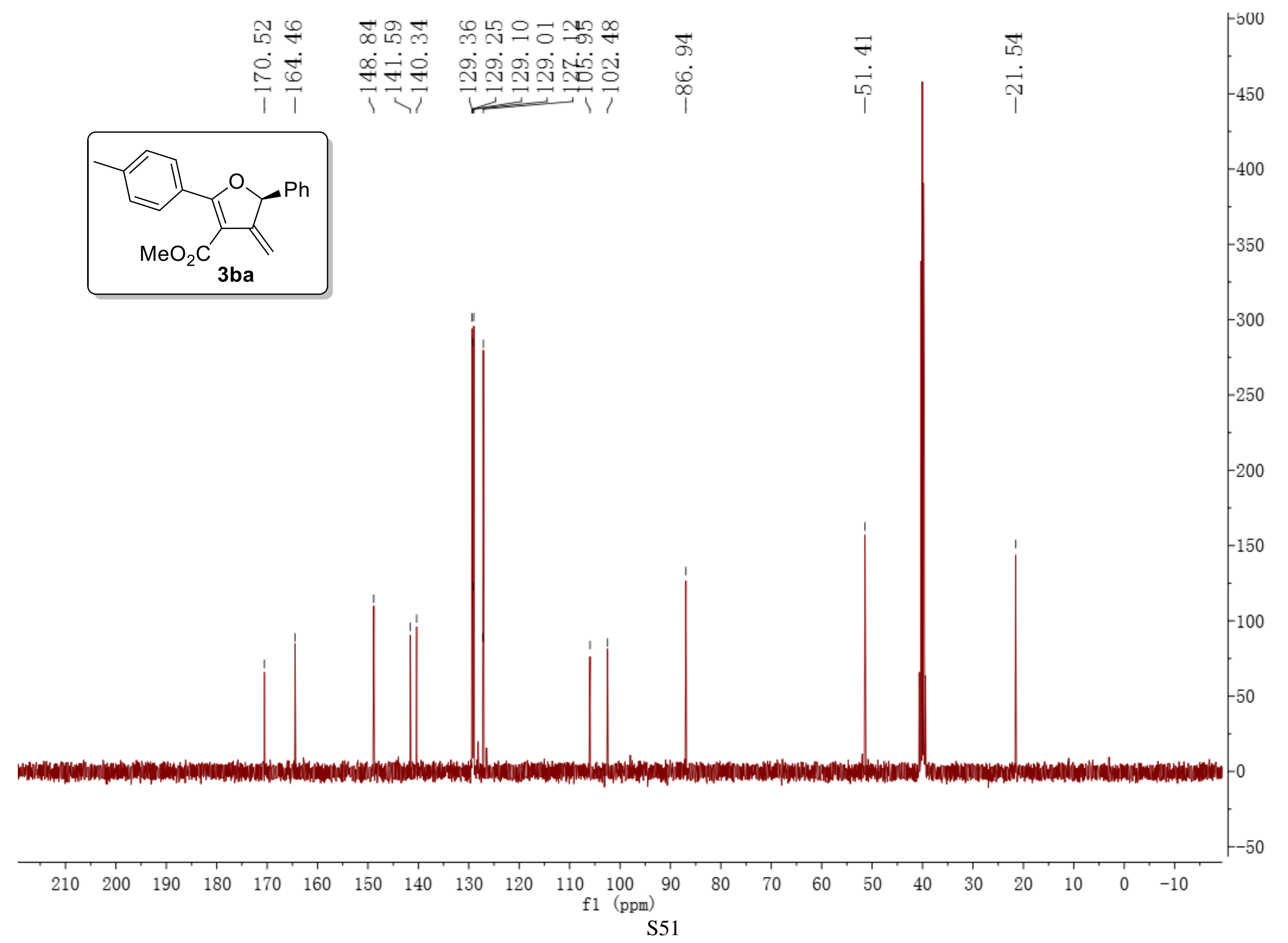




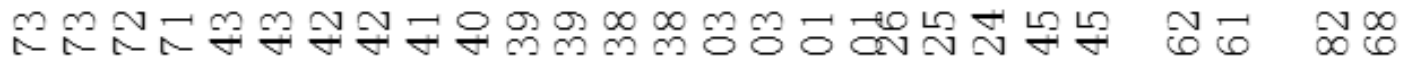

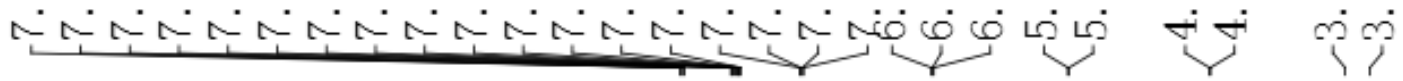




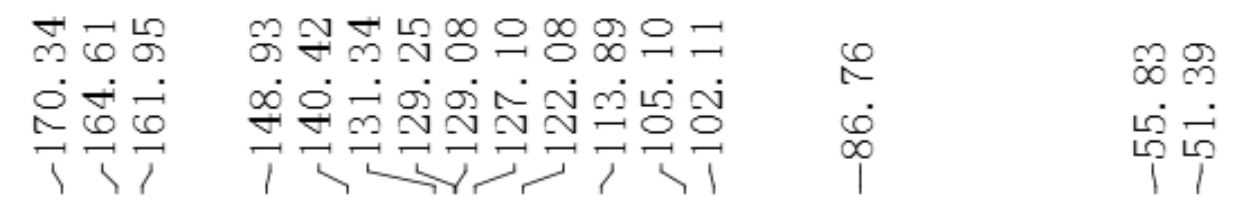

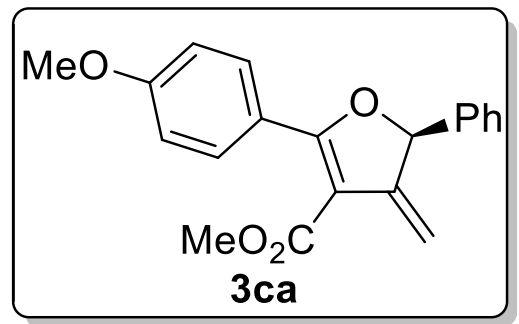




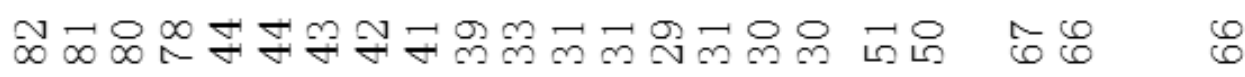

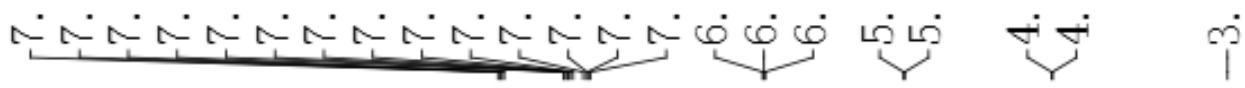

$-5500$

$-5000$

$-4500$

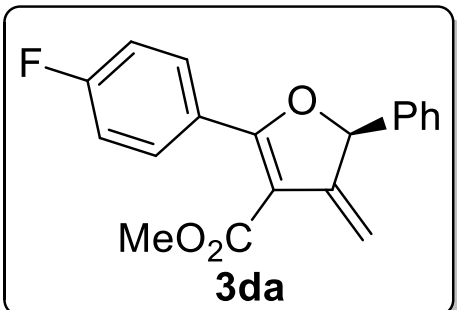

$-4000$

$-3500$

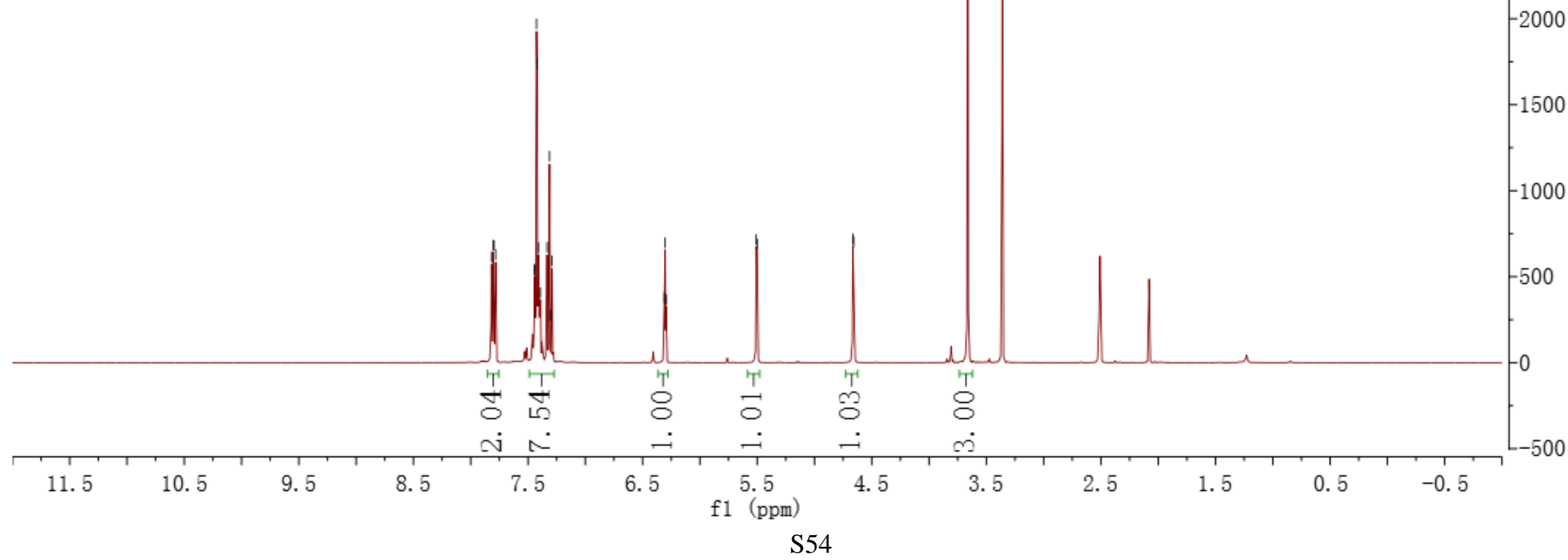


$\infty \propto ⿻ 上$

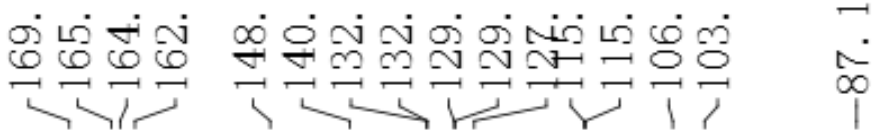

$-850$

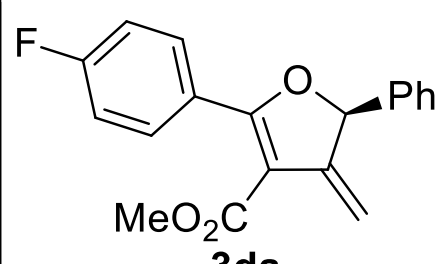


\begin{tabular}{l}
$\stackrel{1}{\circ}$ \\
$\infty$ \\
0 \\
\hdashline \\
\hline
\end{tabular}
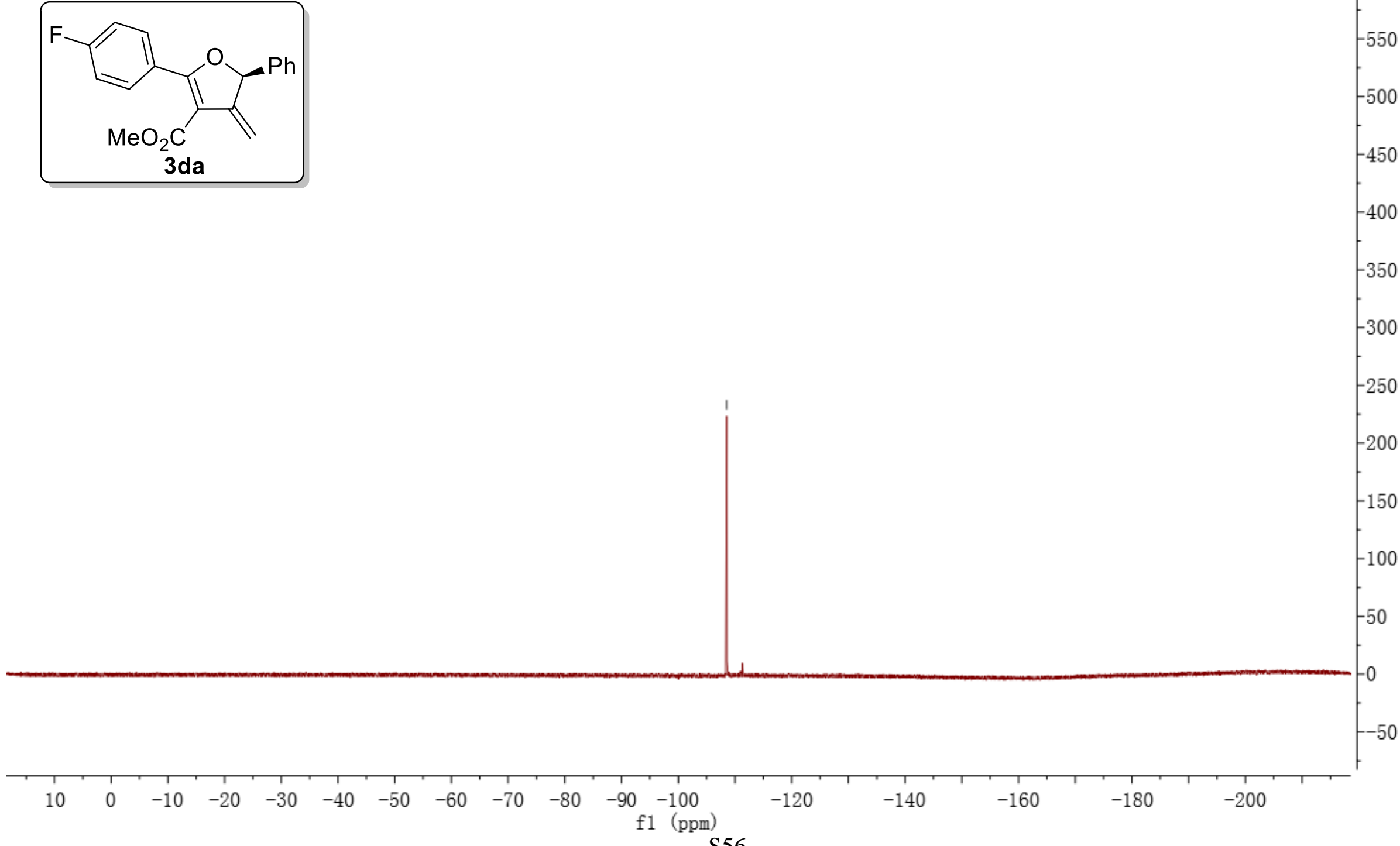


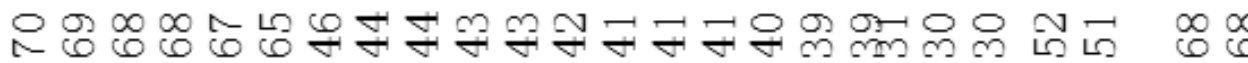

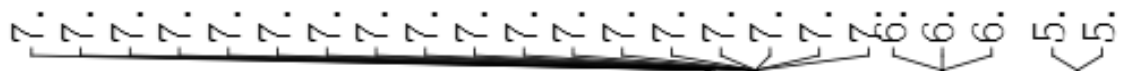
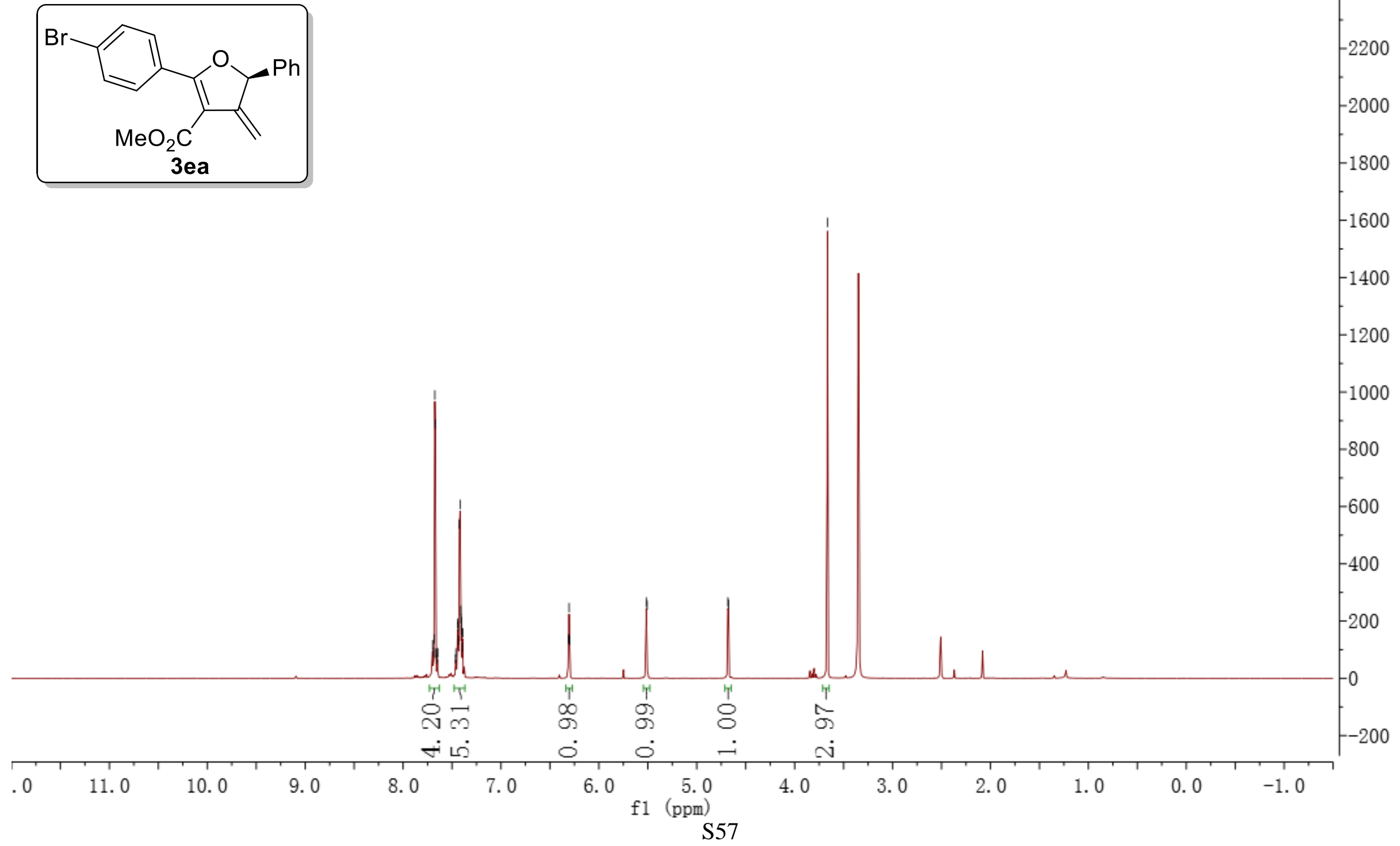


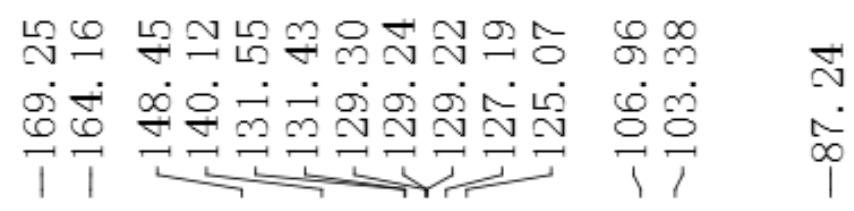
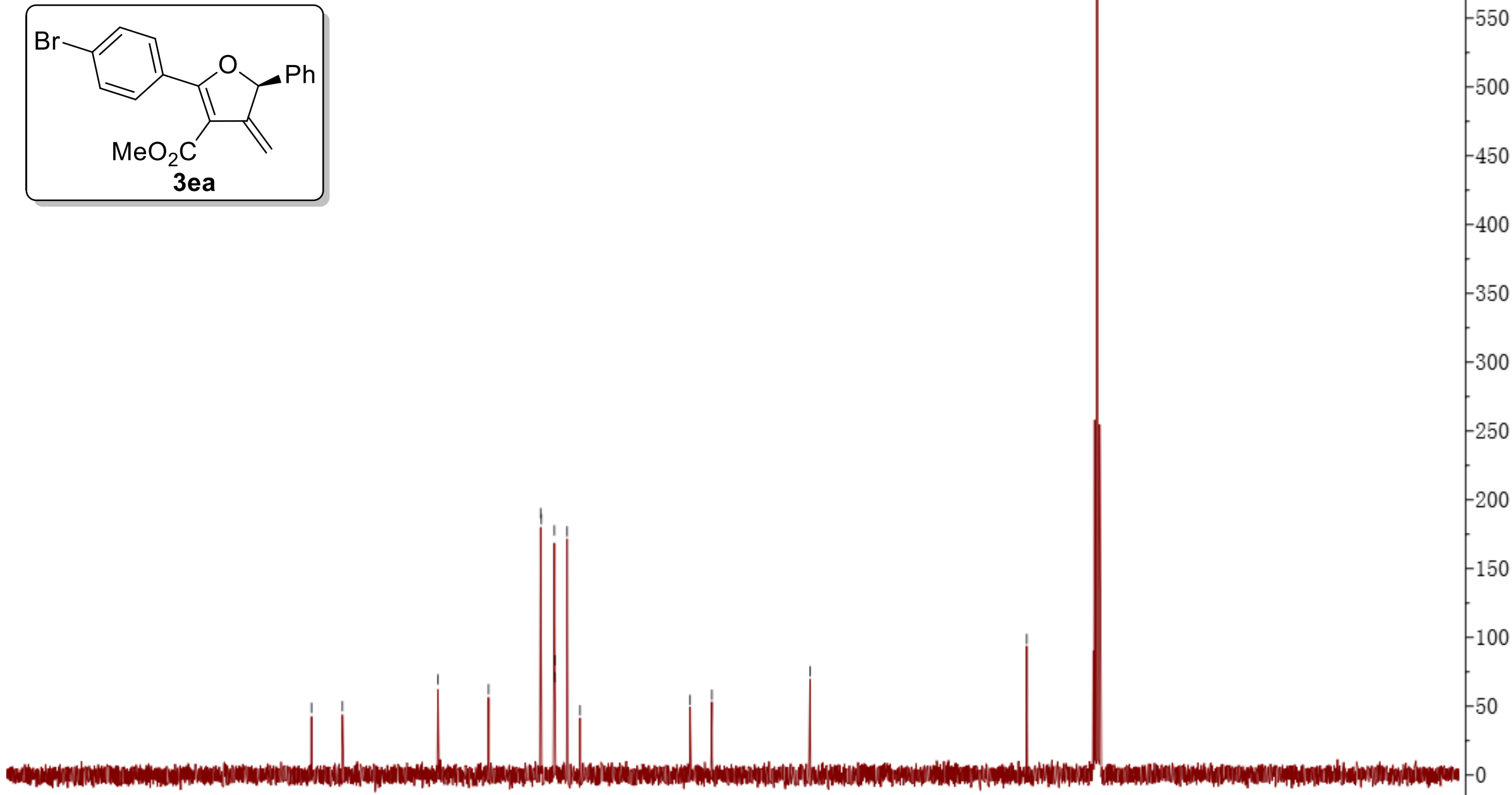


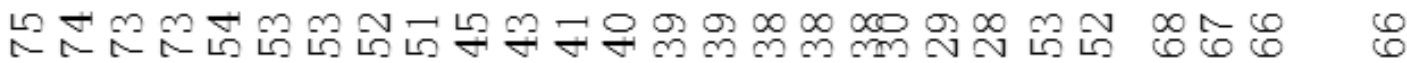

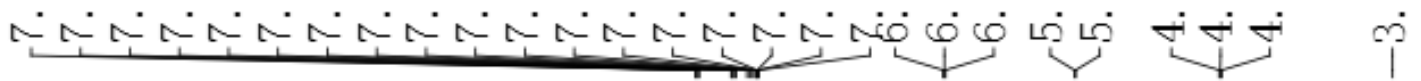
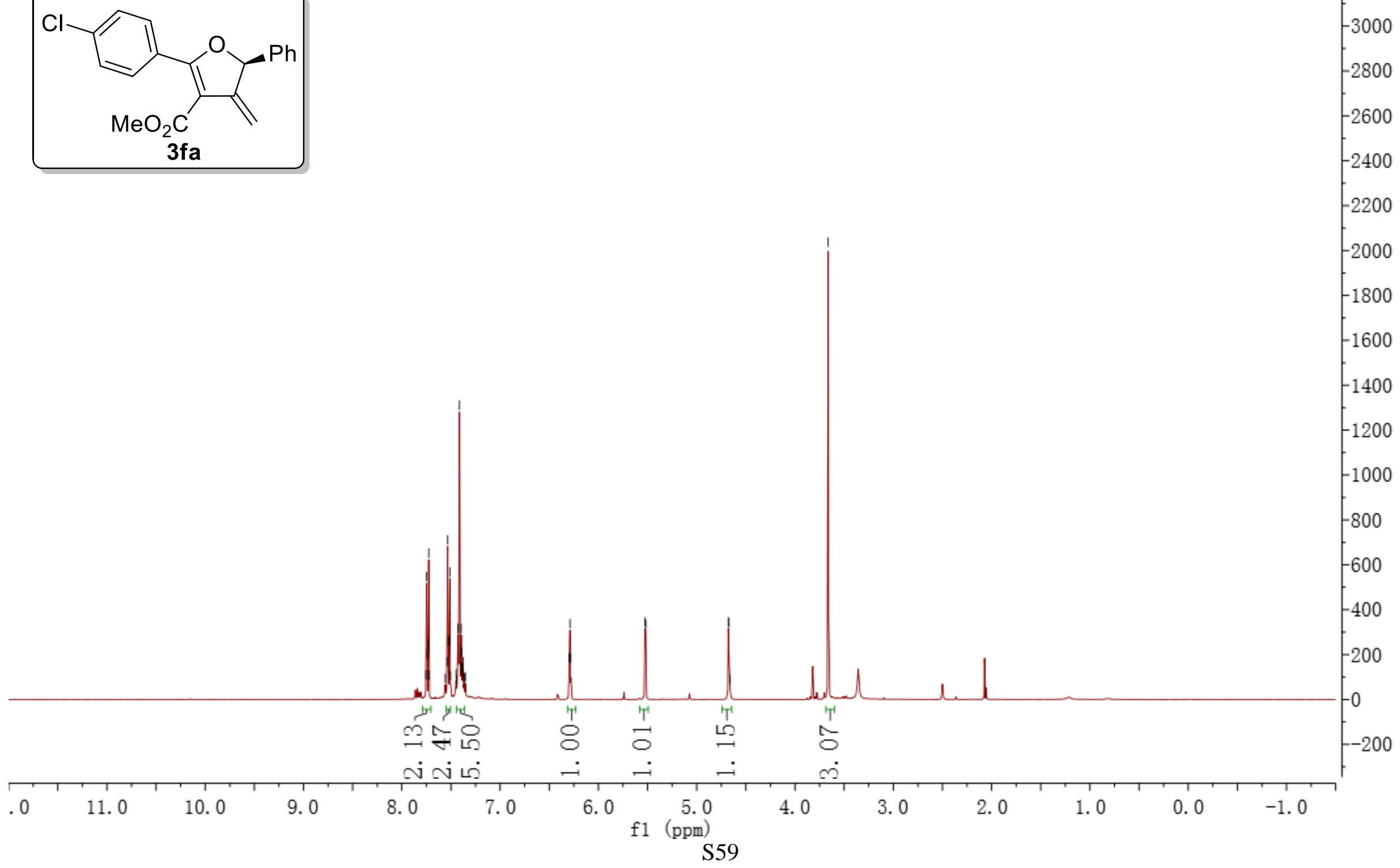


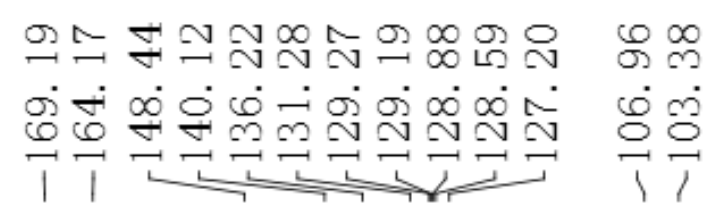

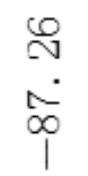

एं

$-600$

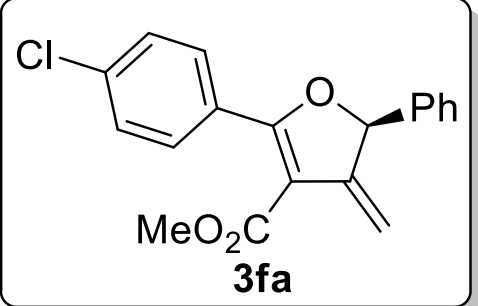



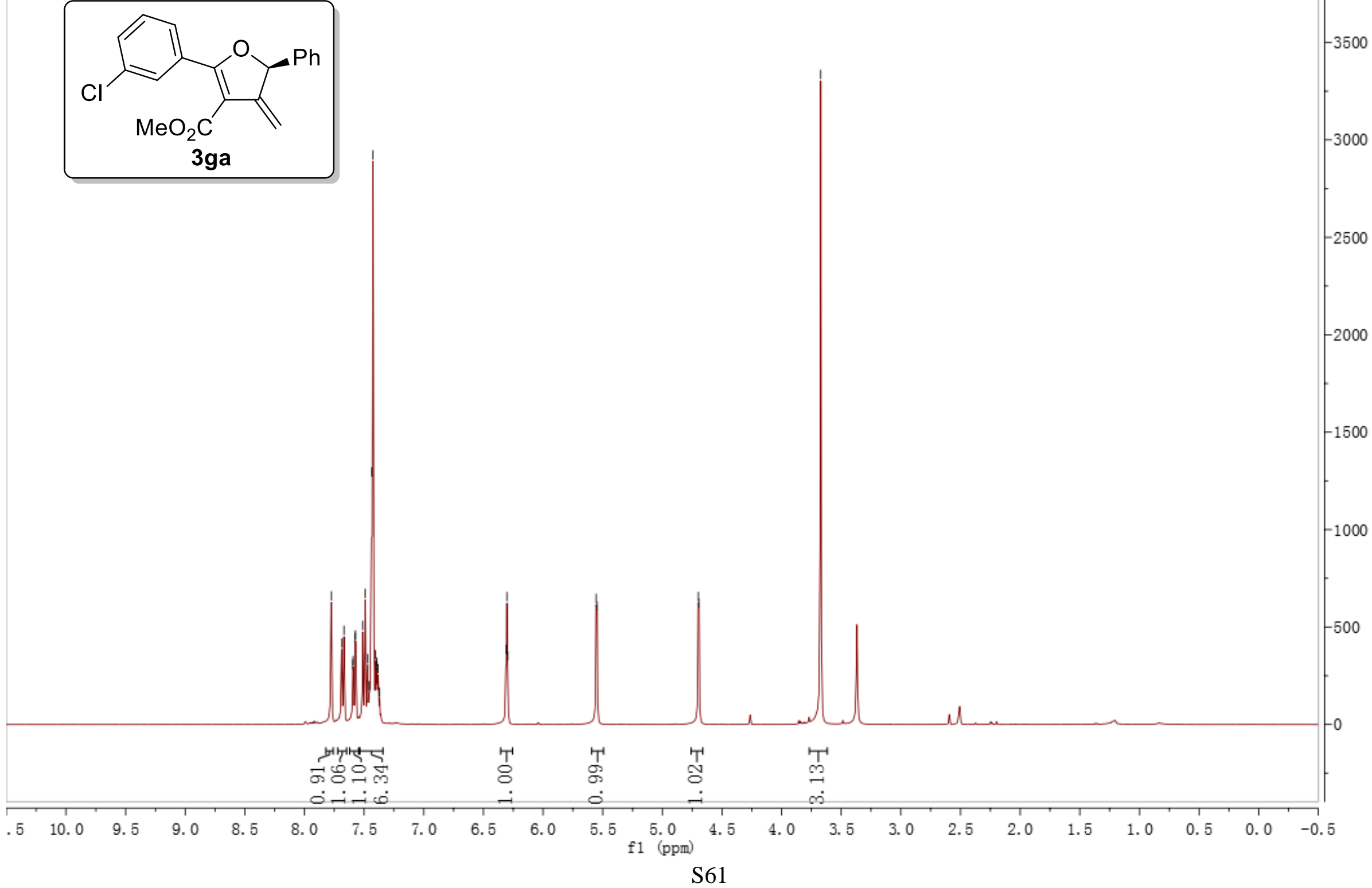


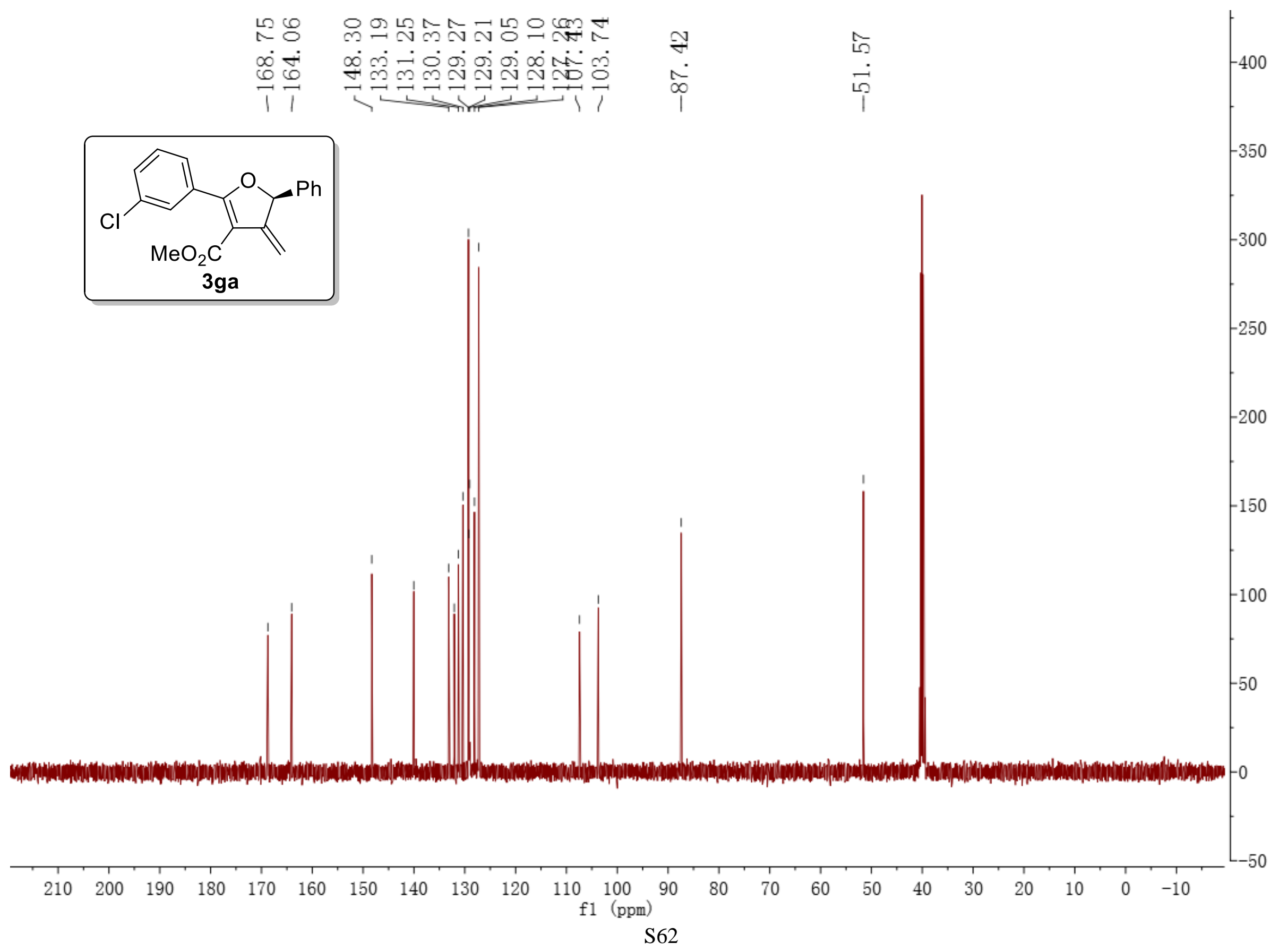




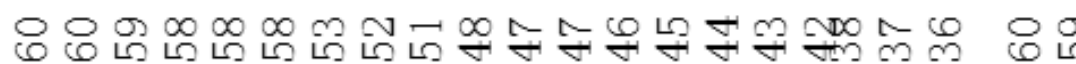

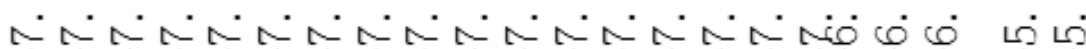

$\begin{array}{ll}\infty \\ 0 & 0\end{array}$

نं

芒
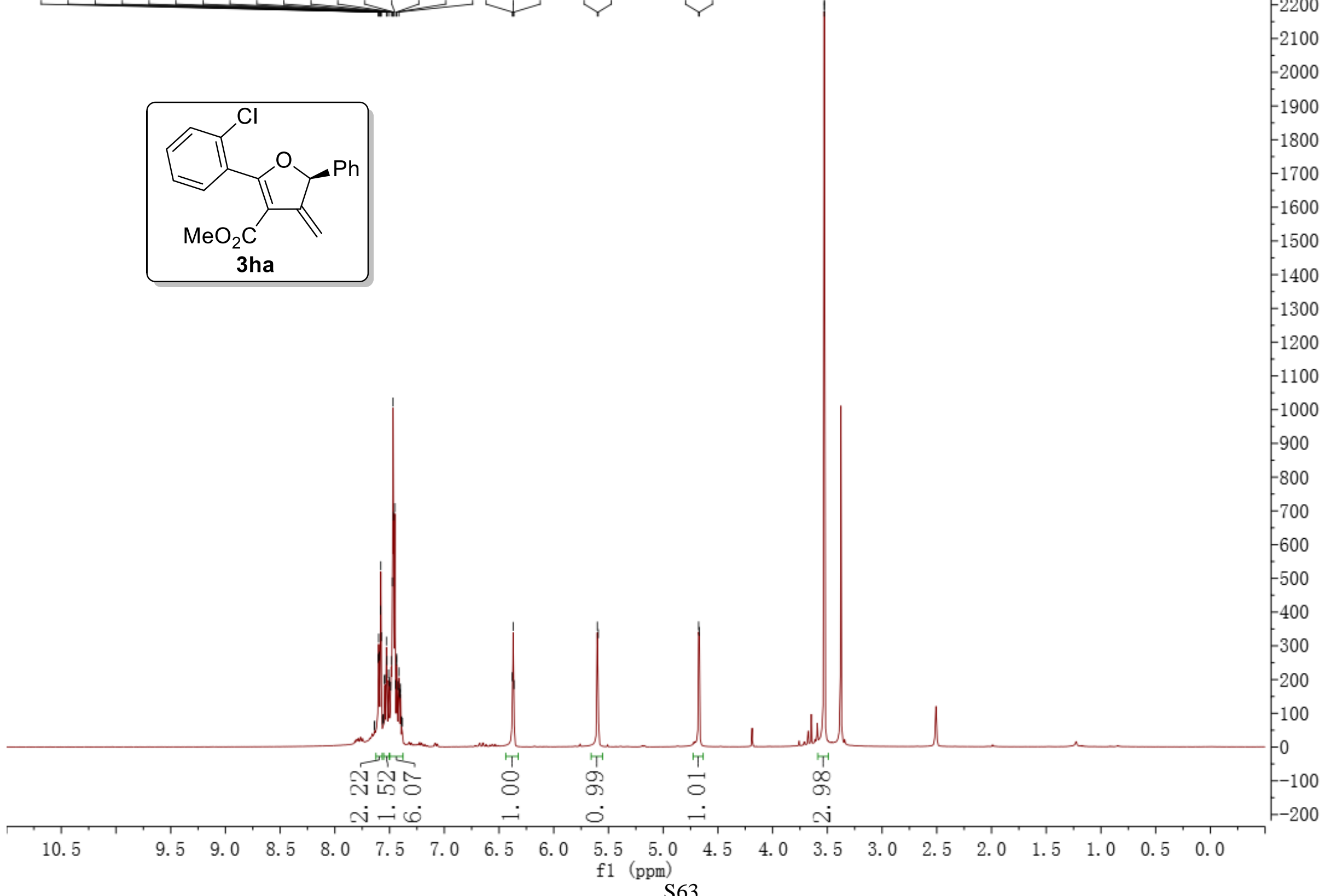


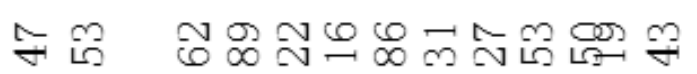 \\ $\begin{array}{ll}\dot{0} & \dot{0} \\ 1\end{array}$ \\ $\sigma$
$\infty$
$\infty$
$\infty$
1}

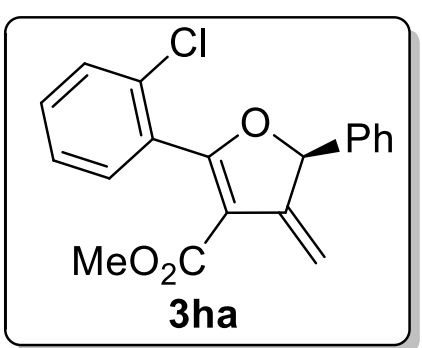

$\stackrel{\infty}{\stackrel{\infty}{\dot{p}}}$

$-380$

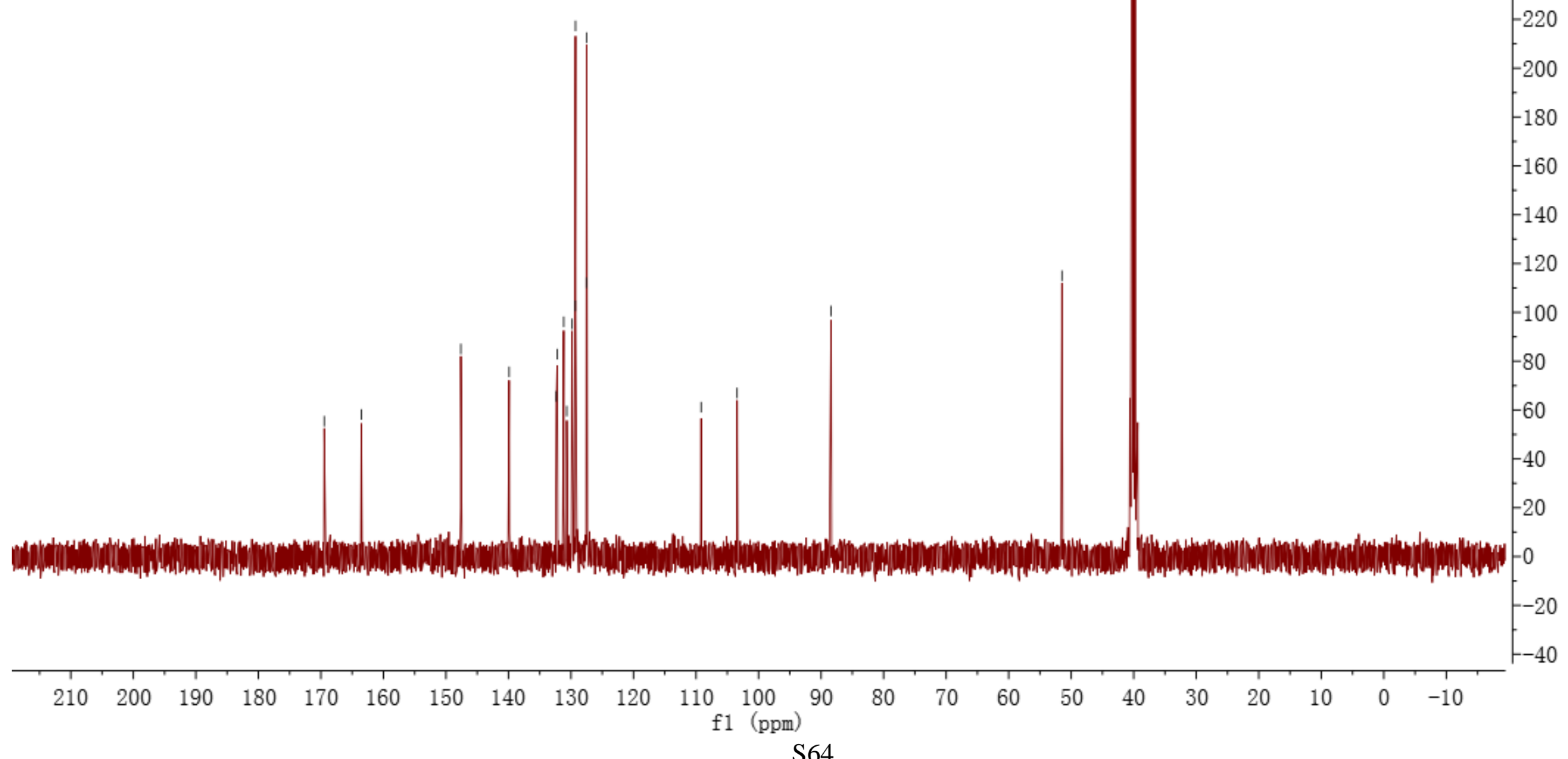




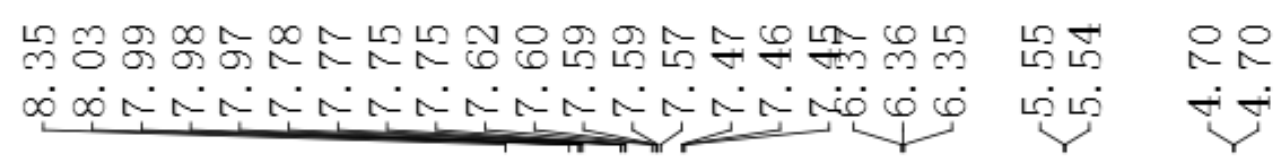

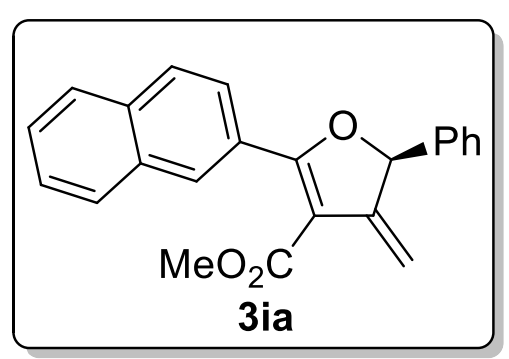

$\$$

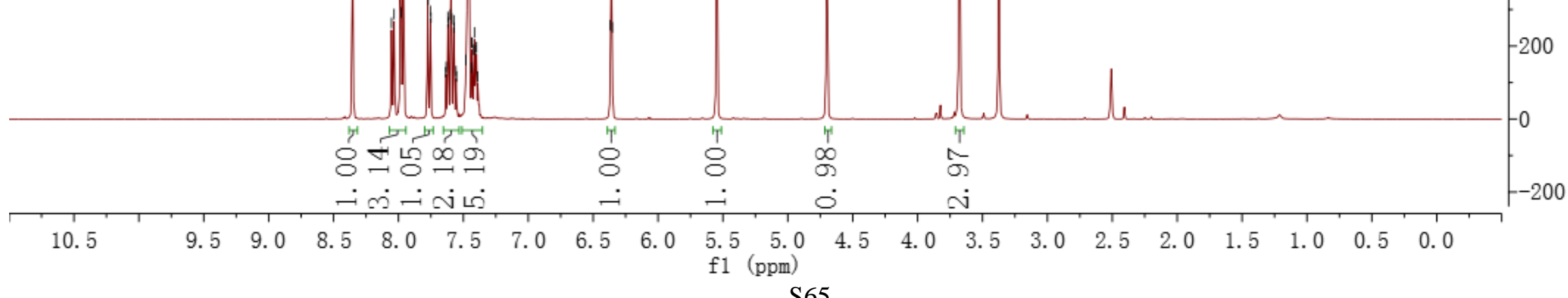


궁

부

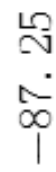

।
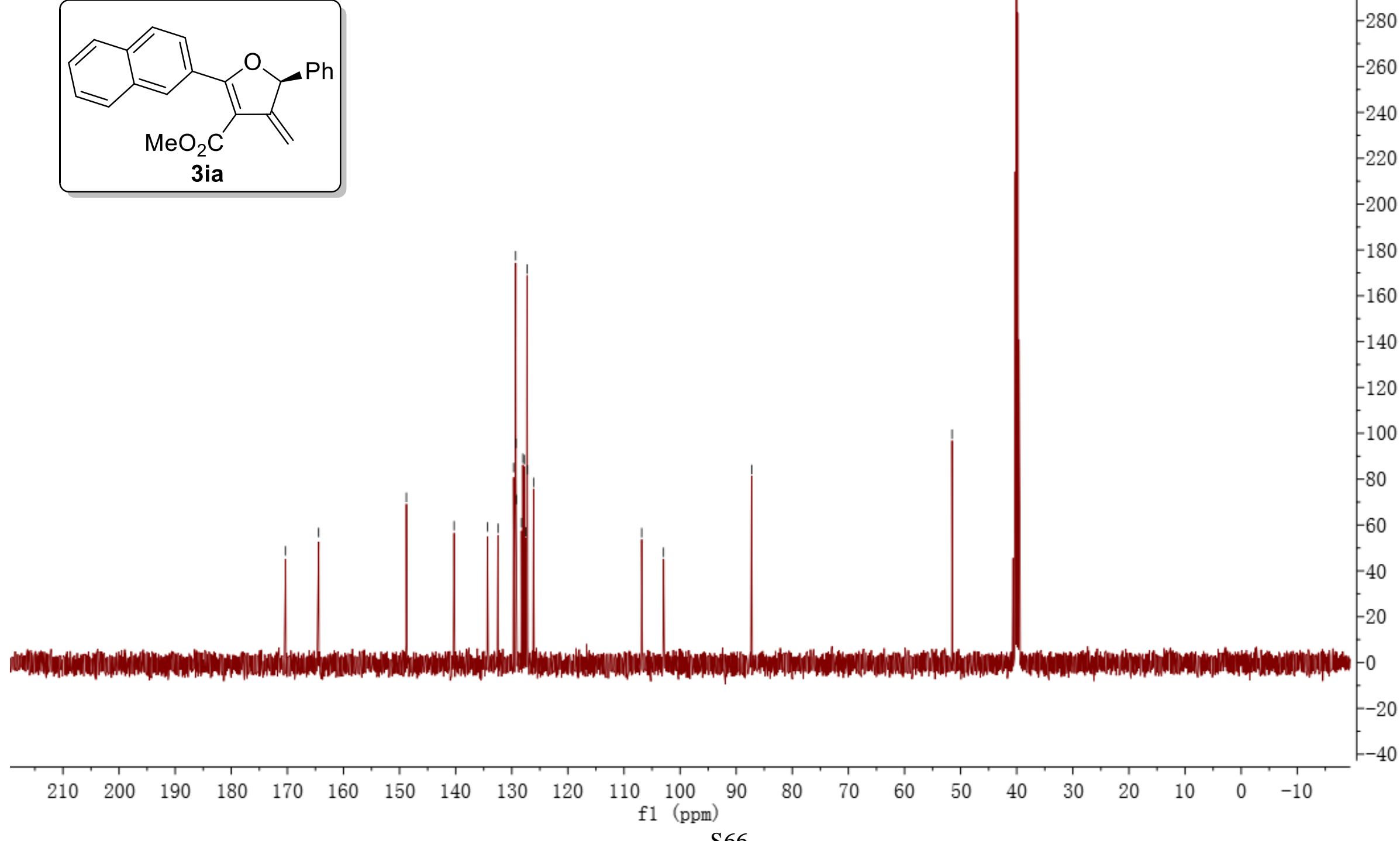


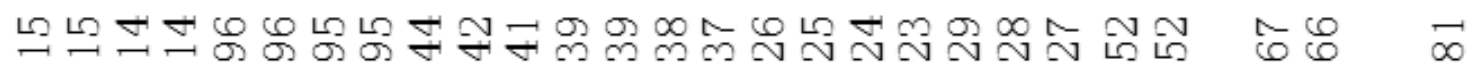

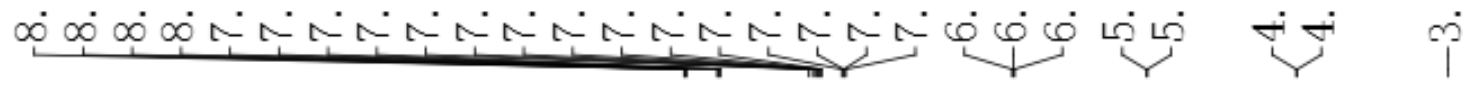
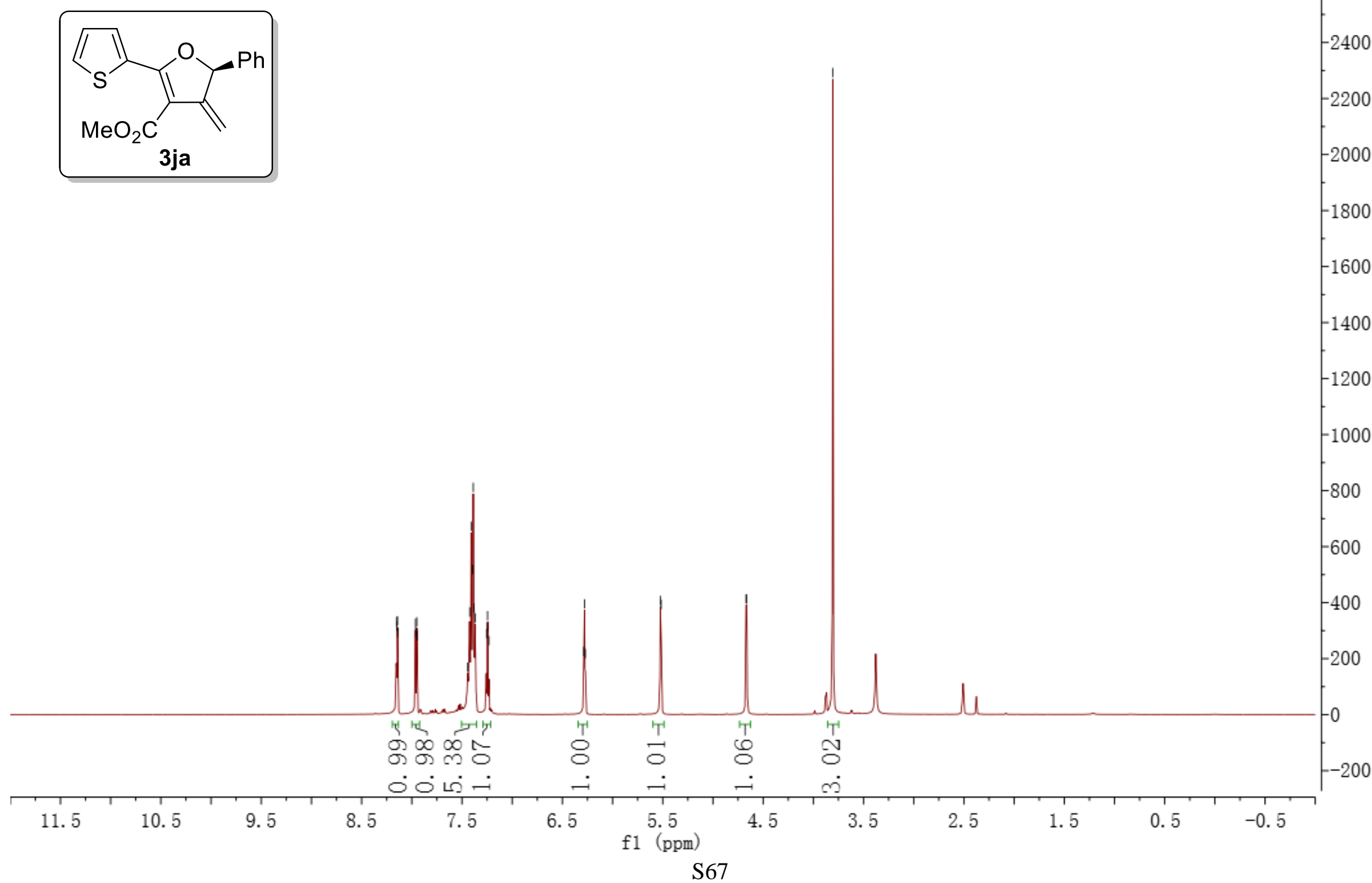


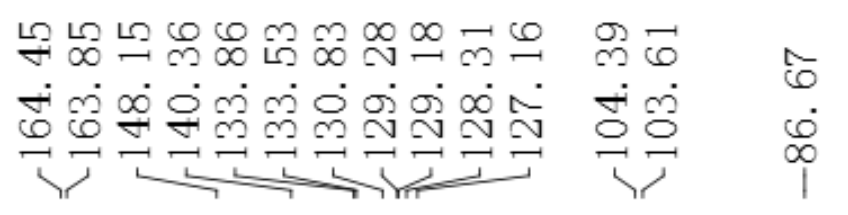

$-850$

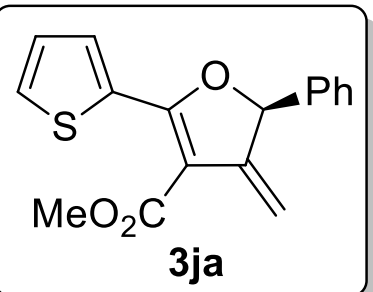




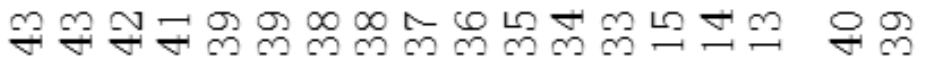

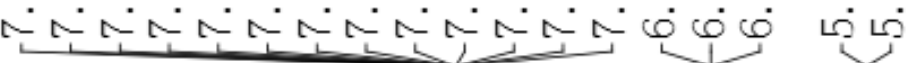

$$
\begin{aligned}
& \stackrel{\infty}{2} \\
& \text { تं }
\end{aligned}
$$

m)
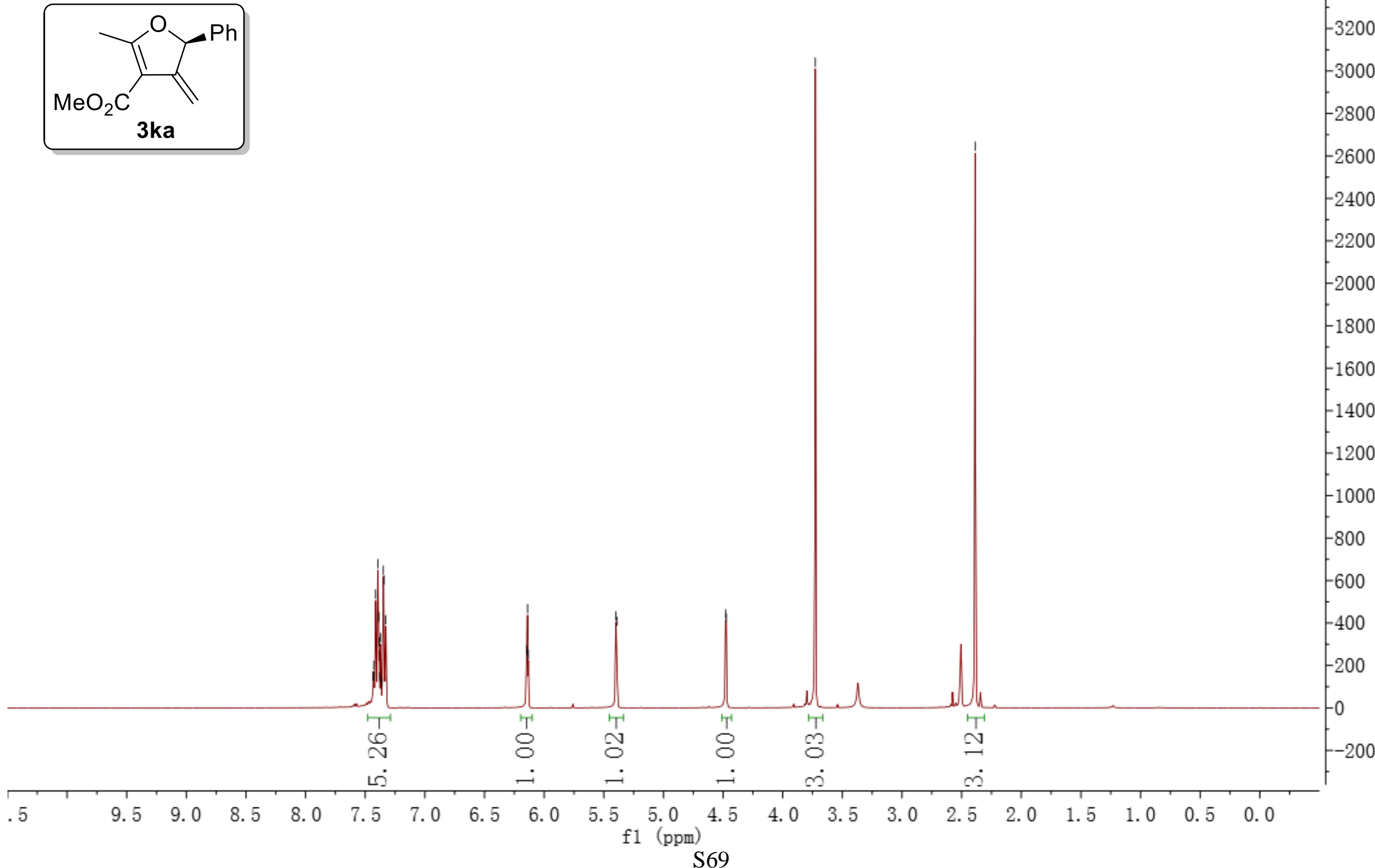


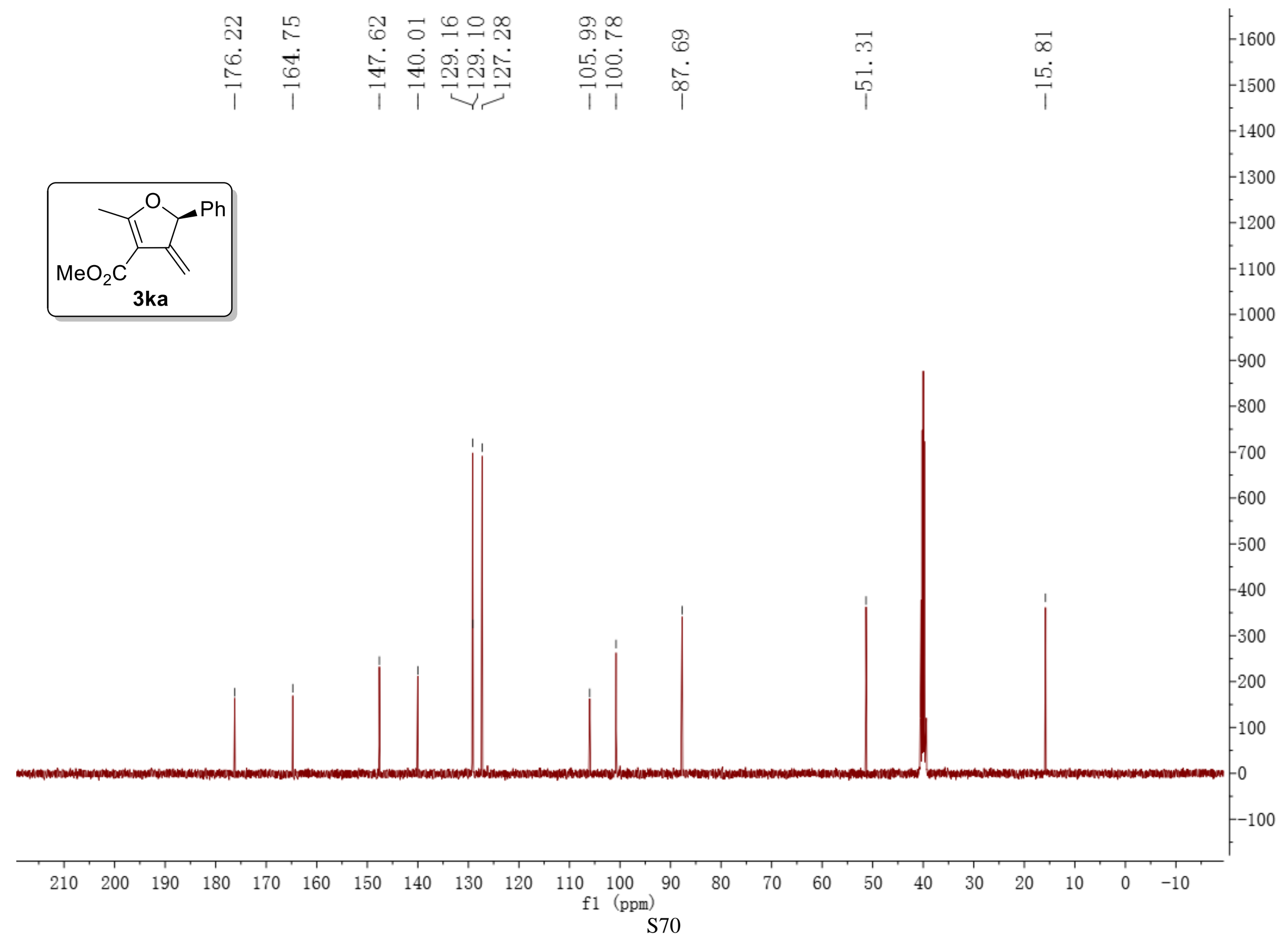




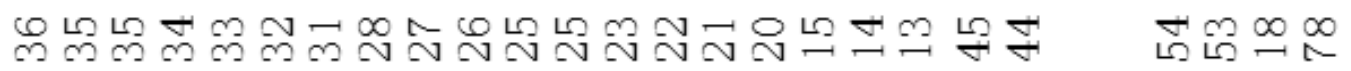

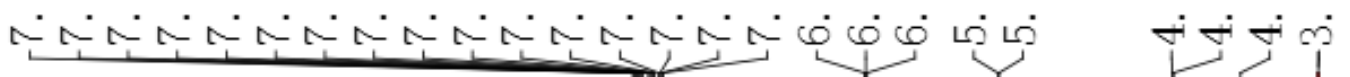
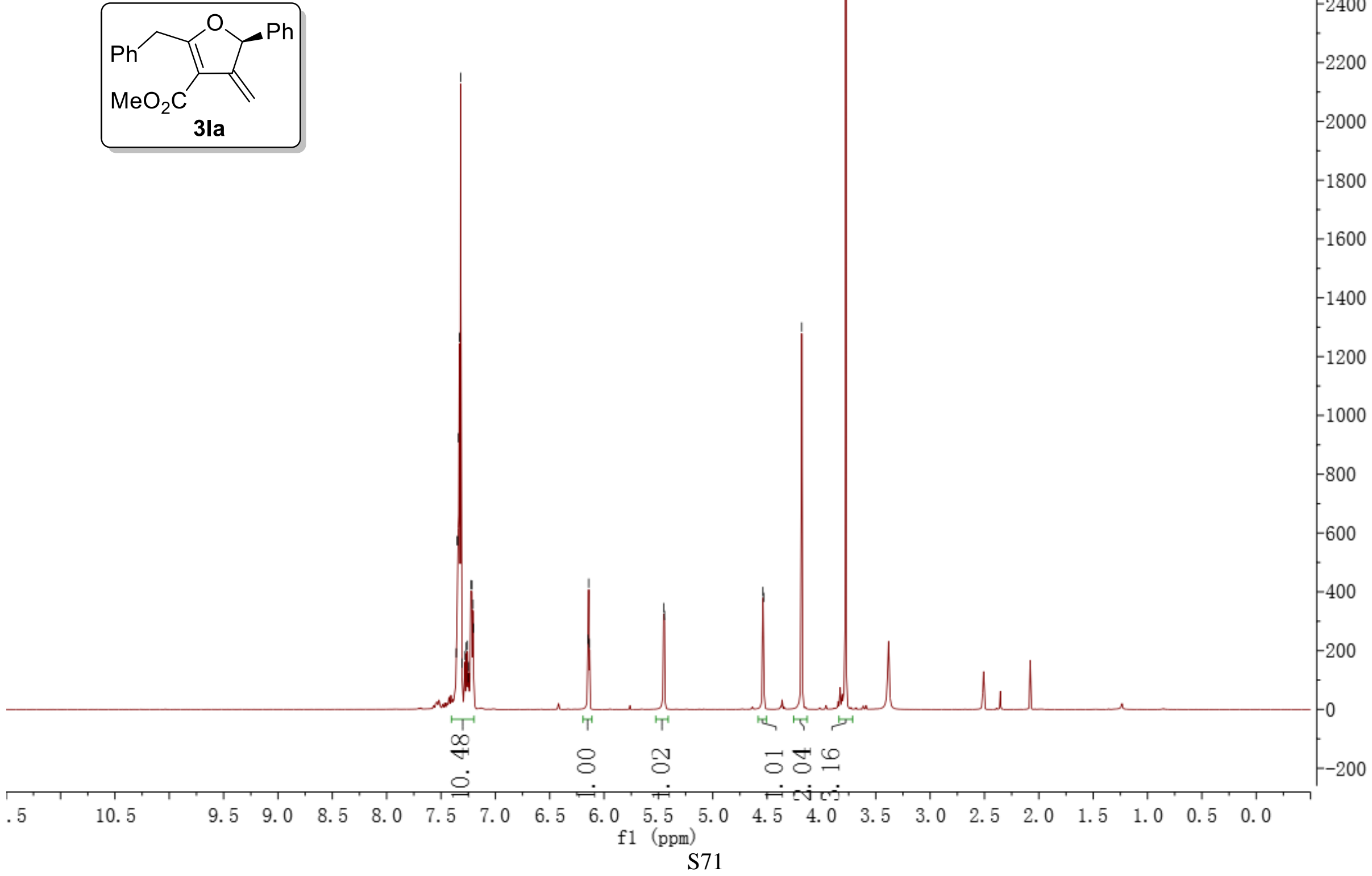


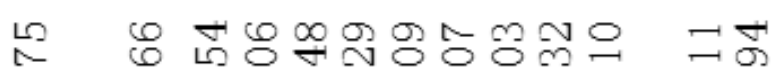

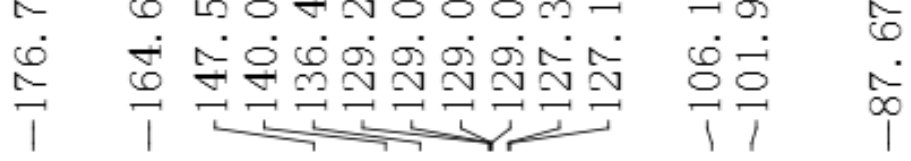
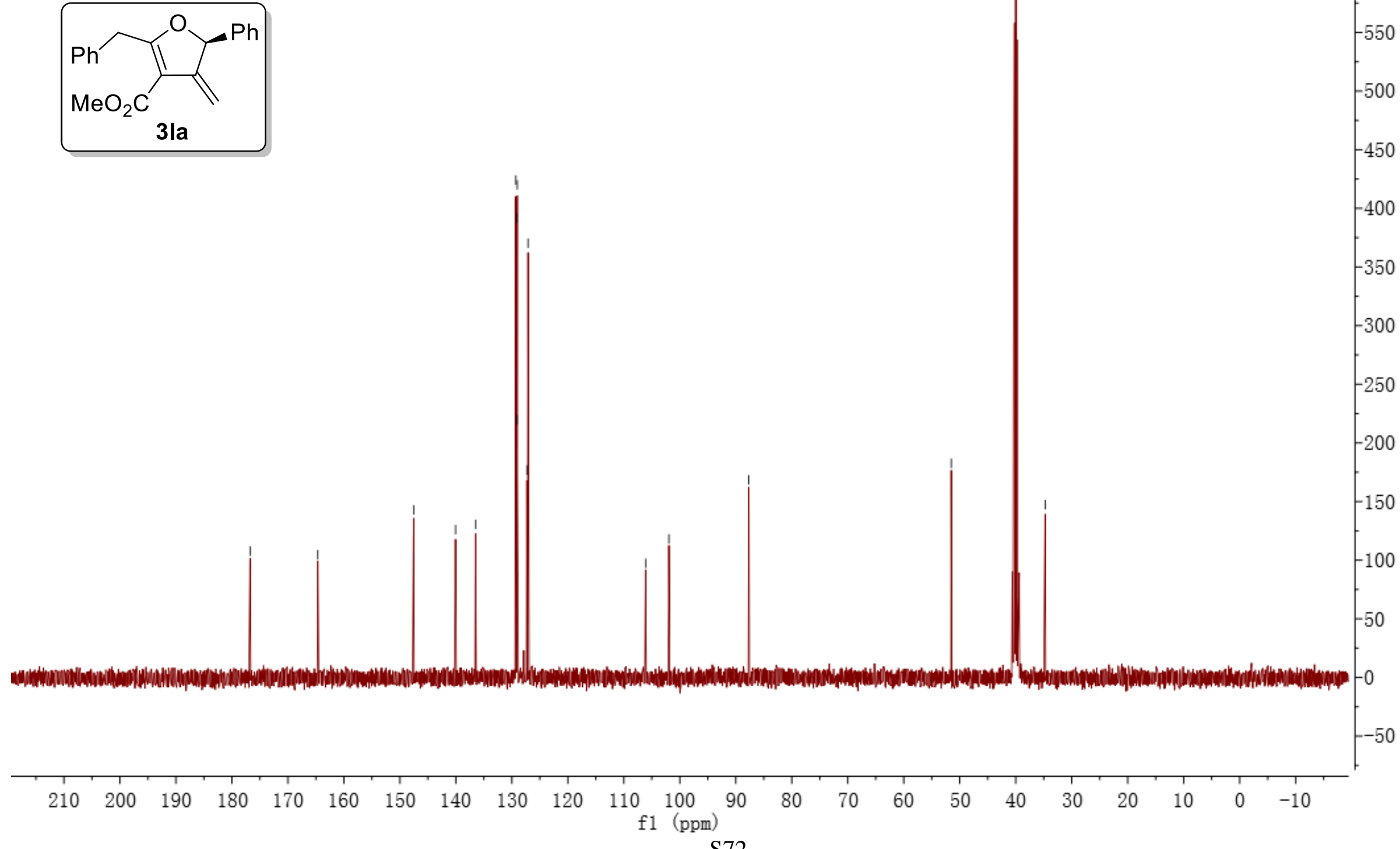


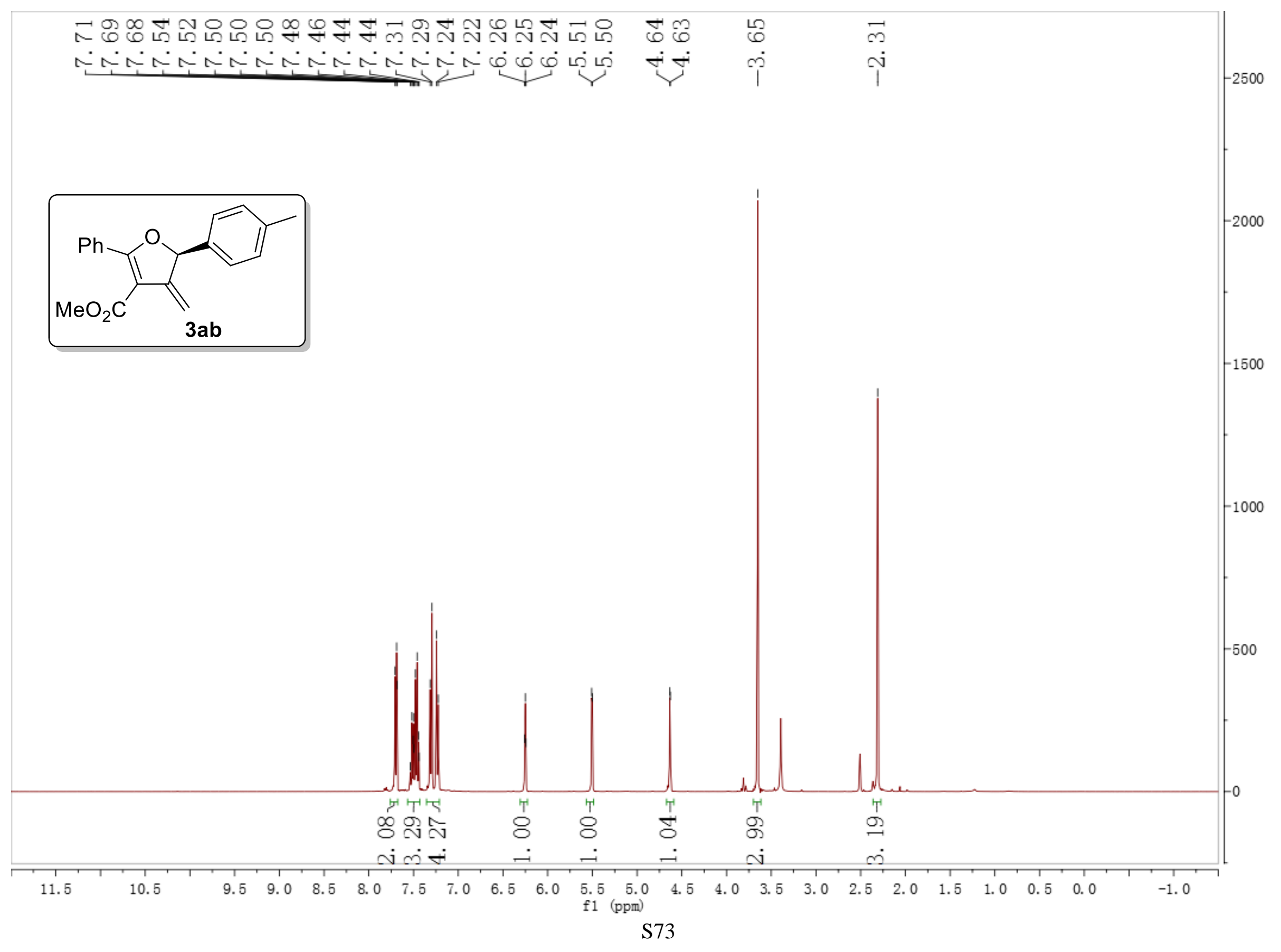




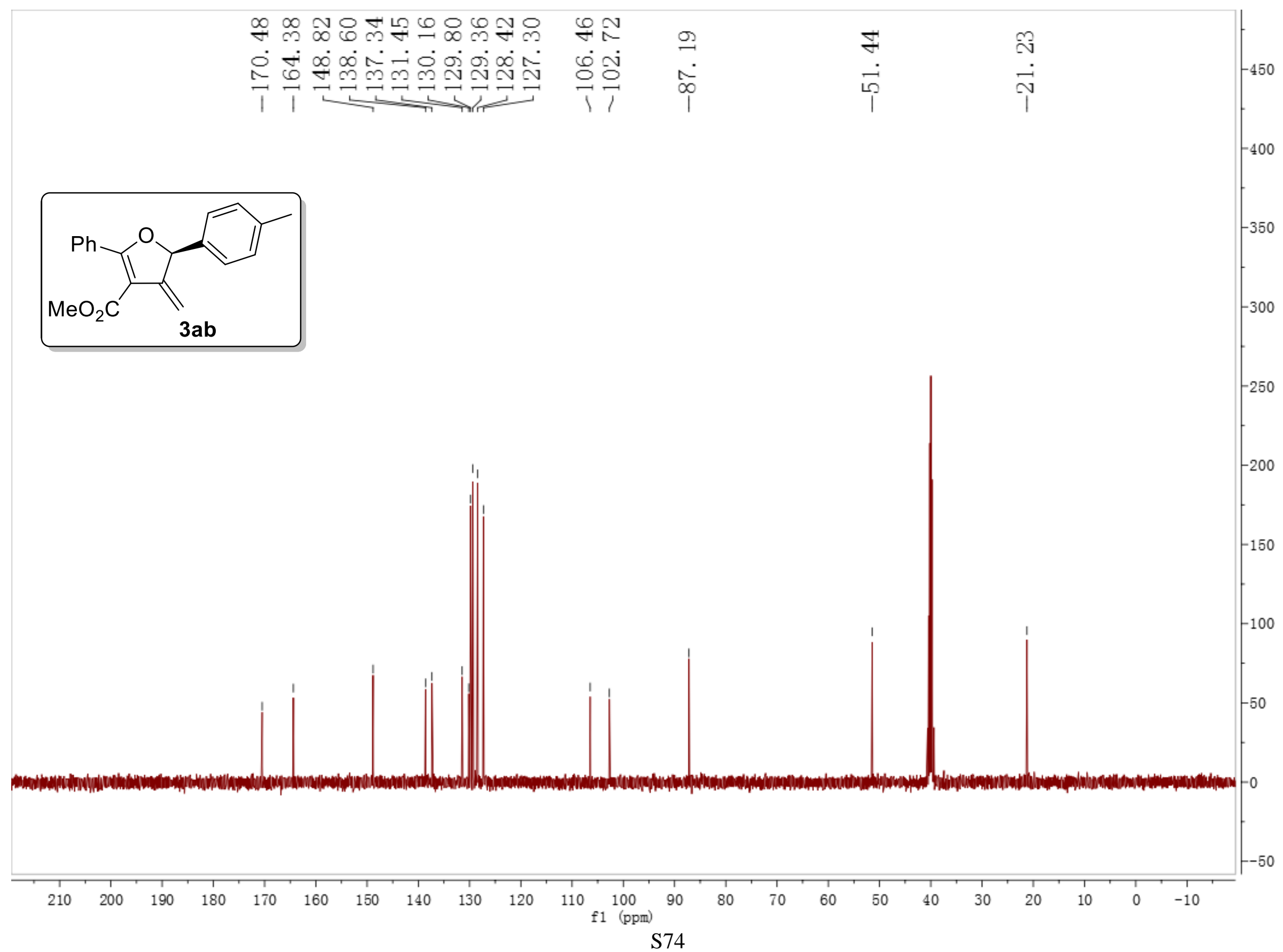




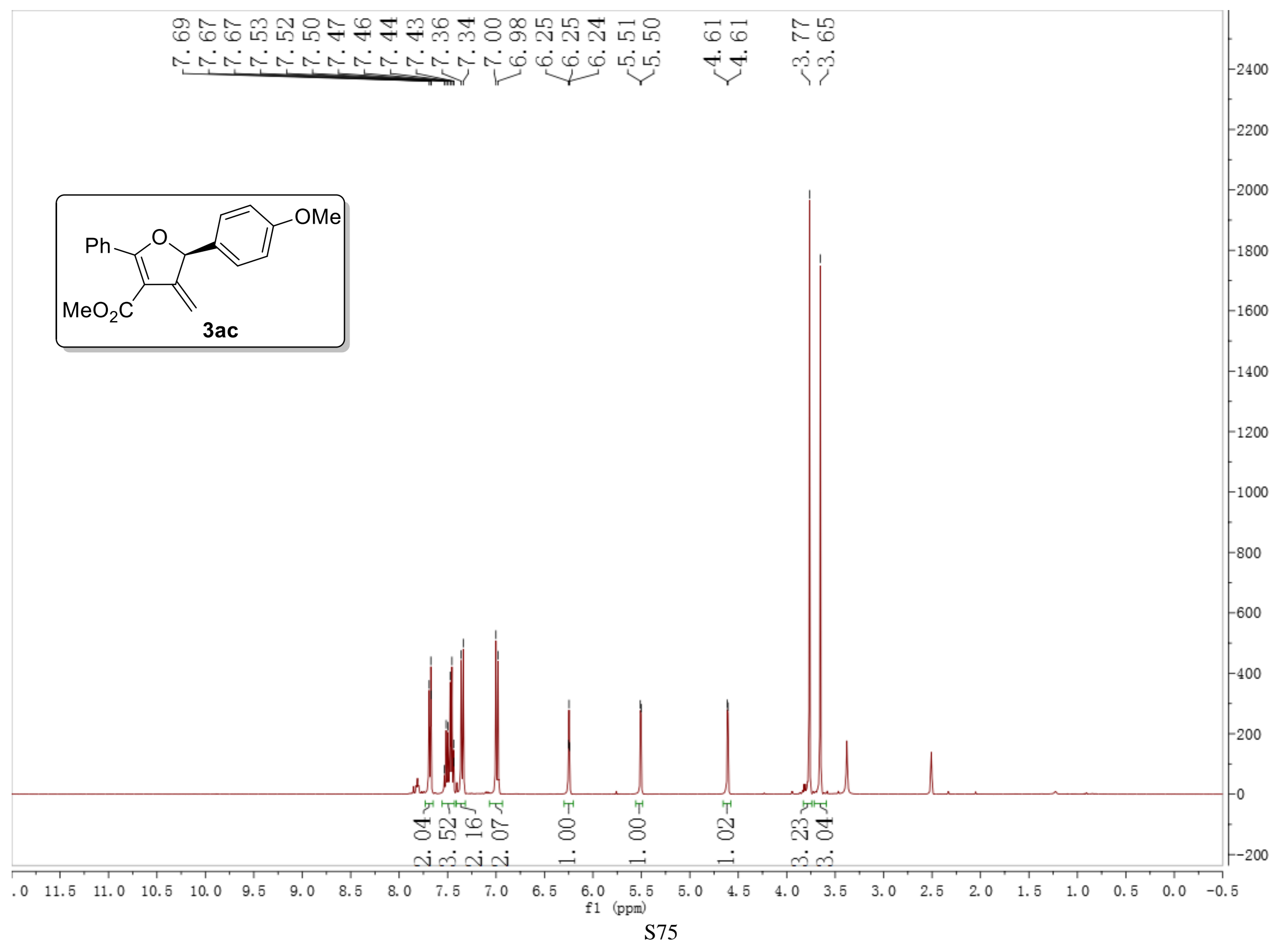




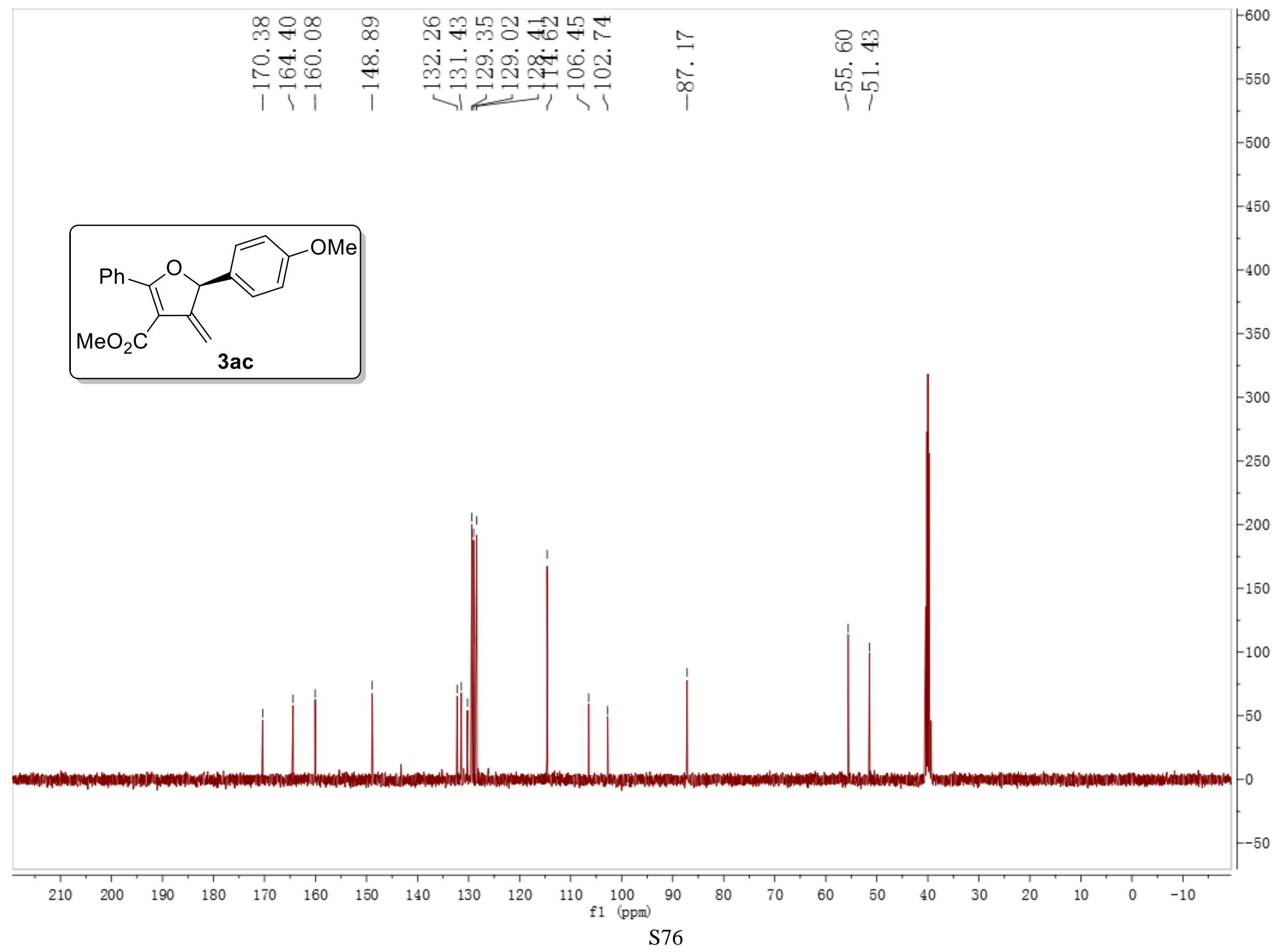




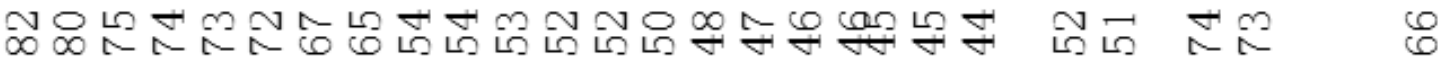

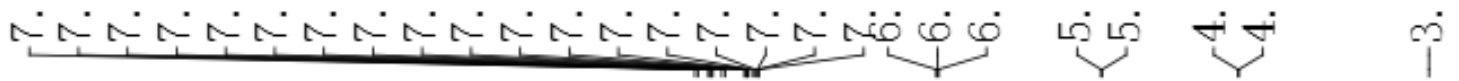
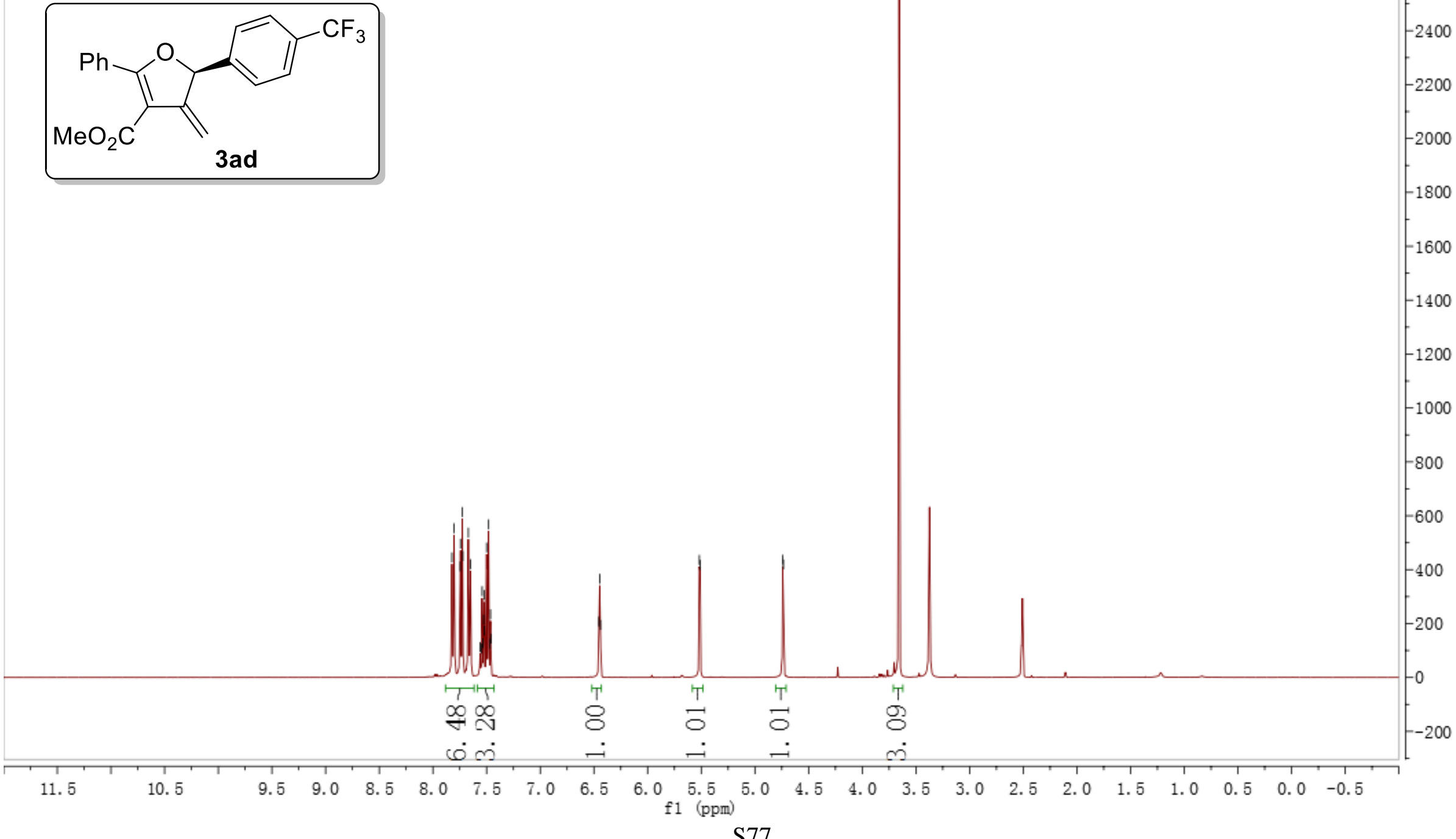


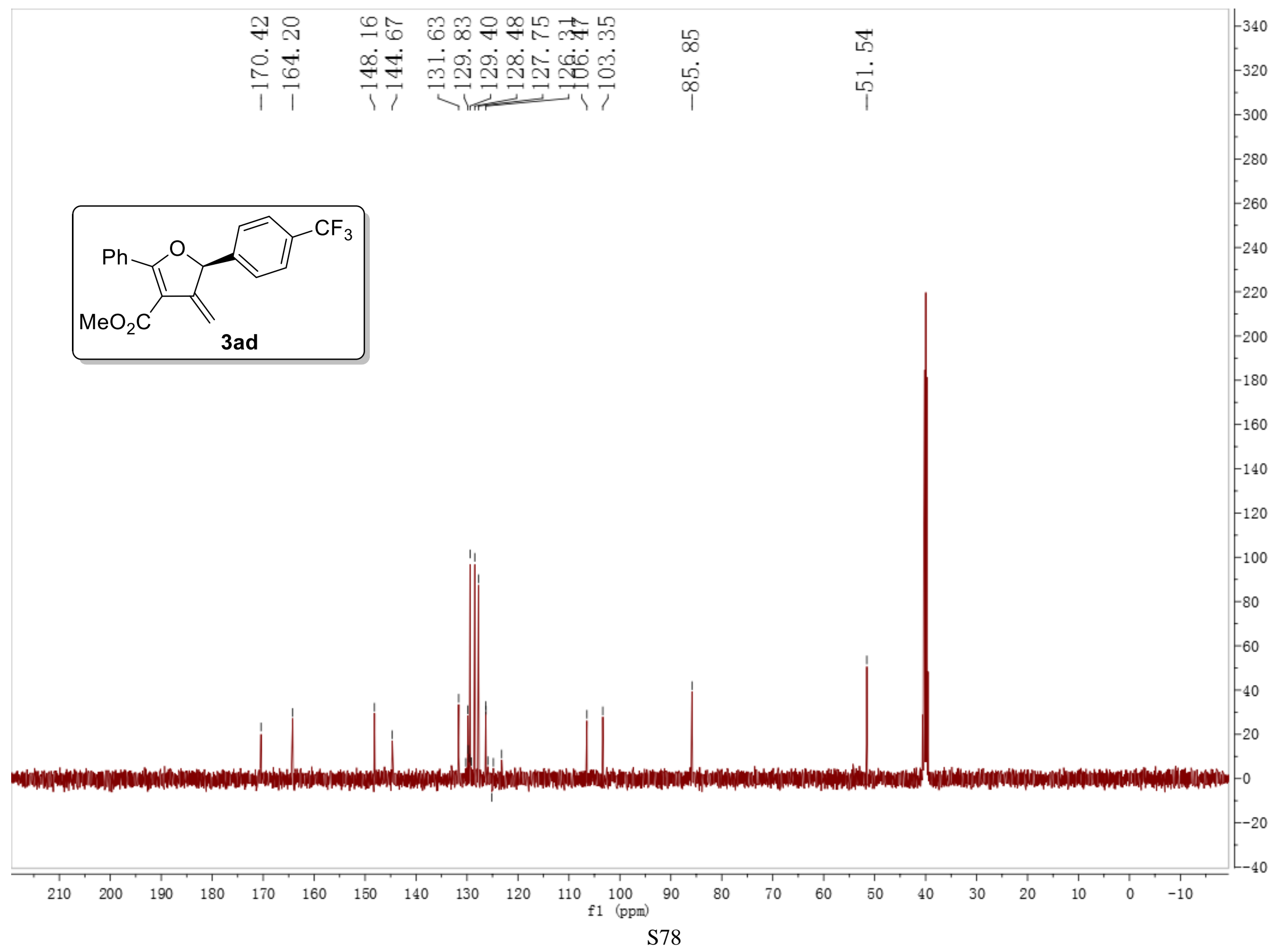



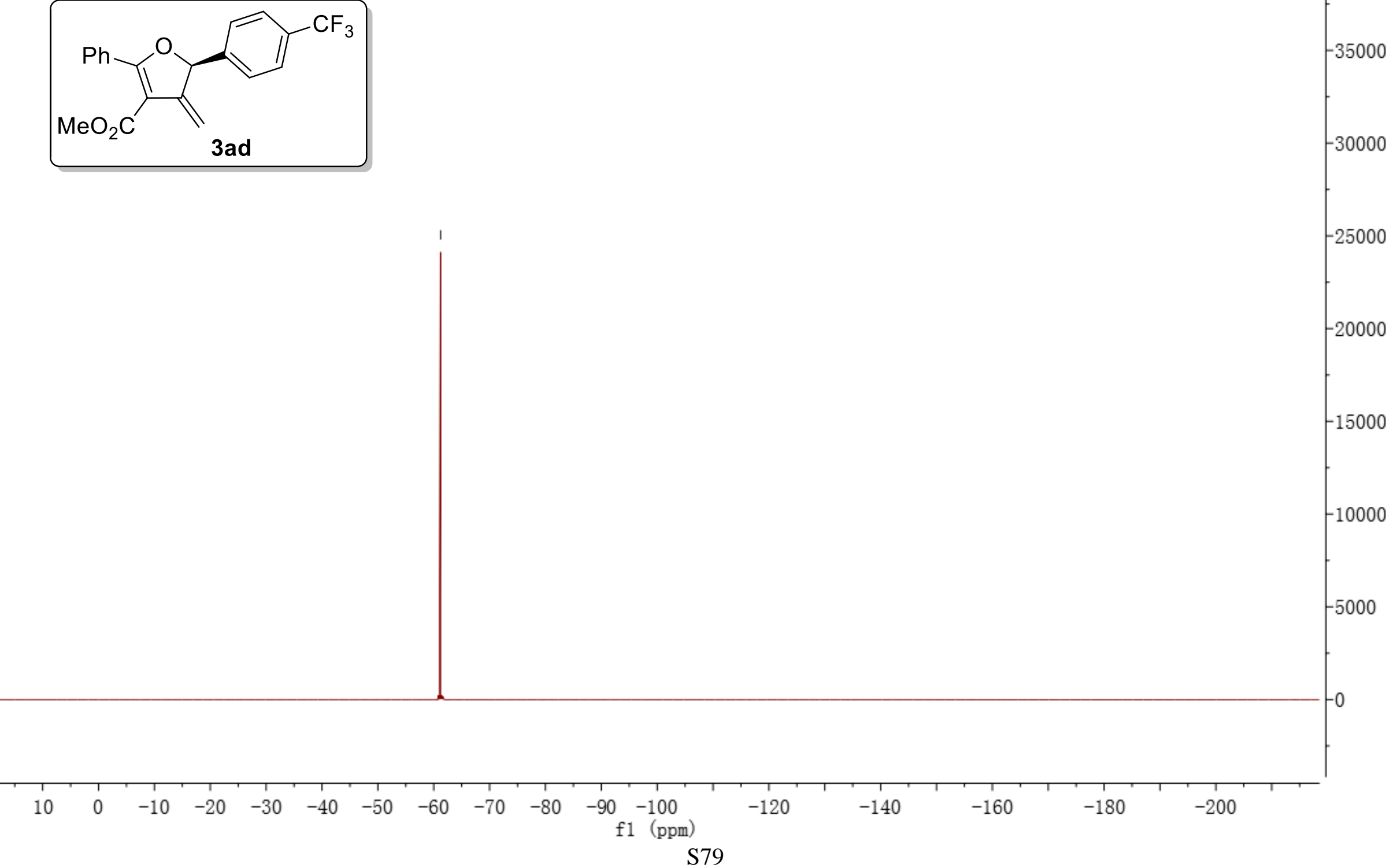


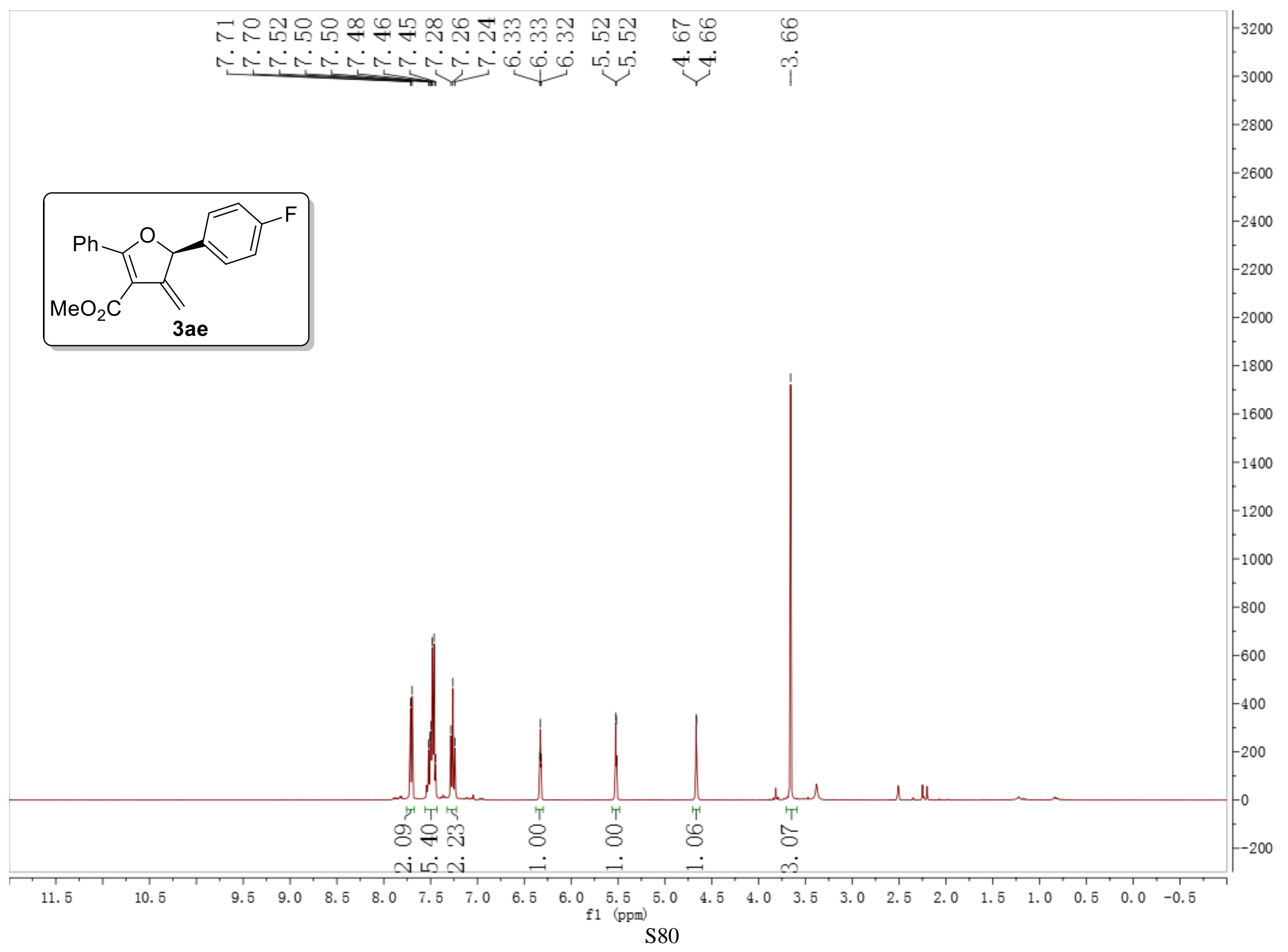




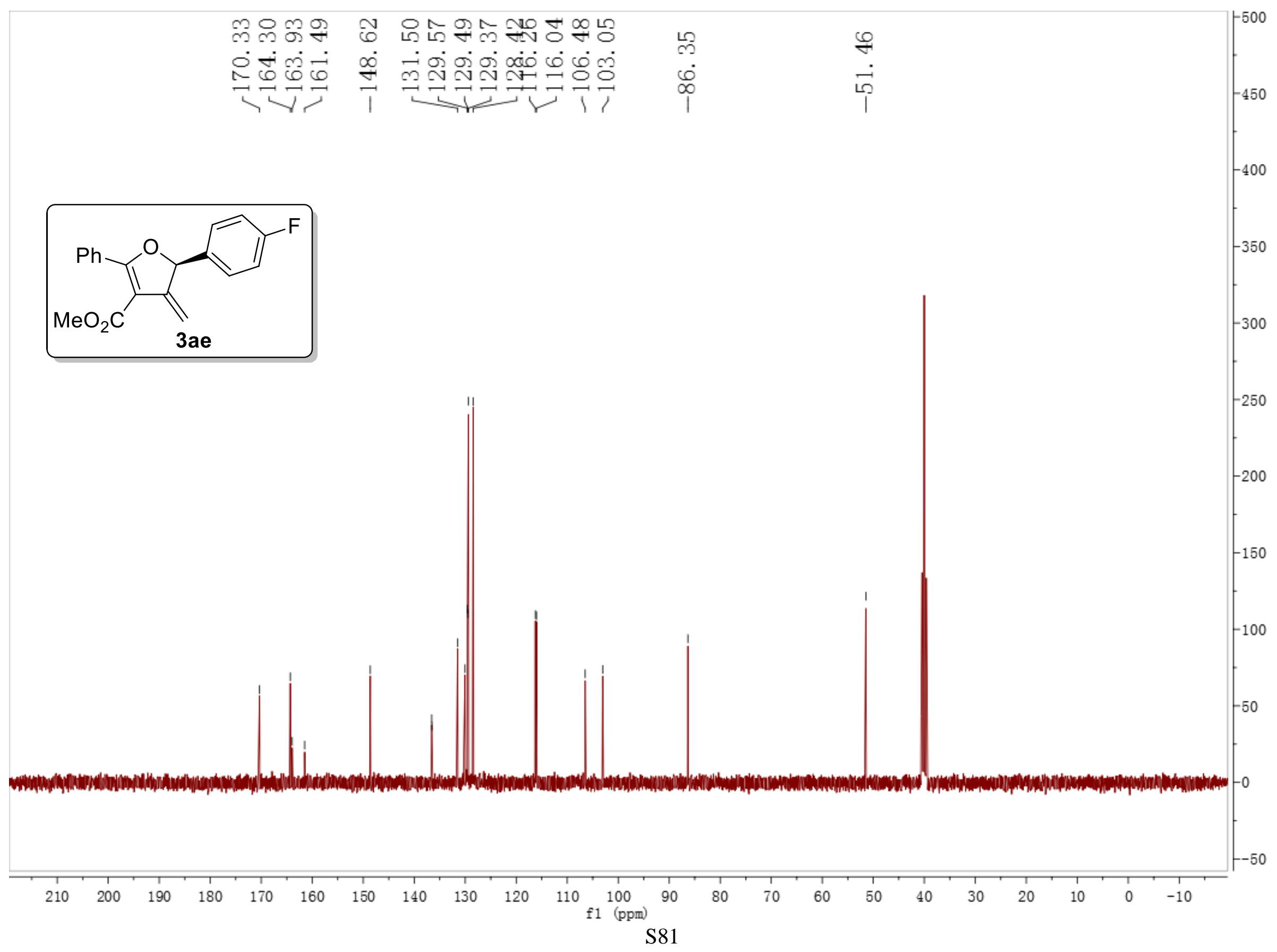




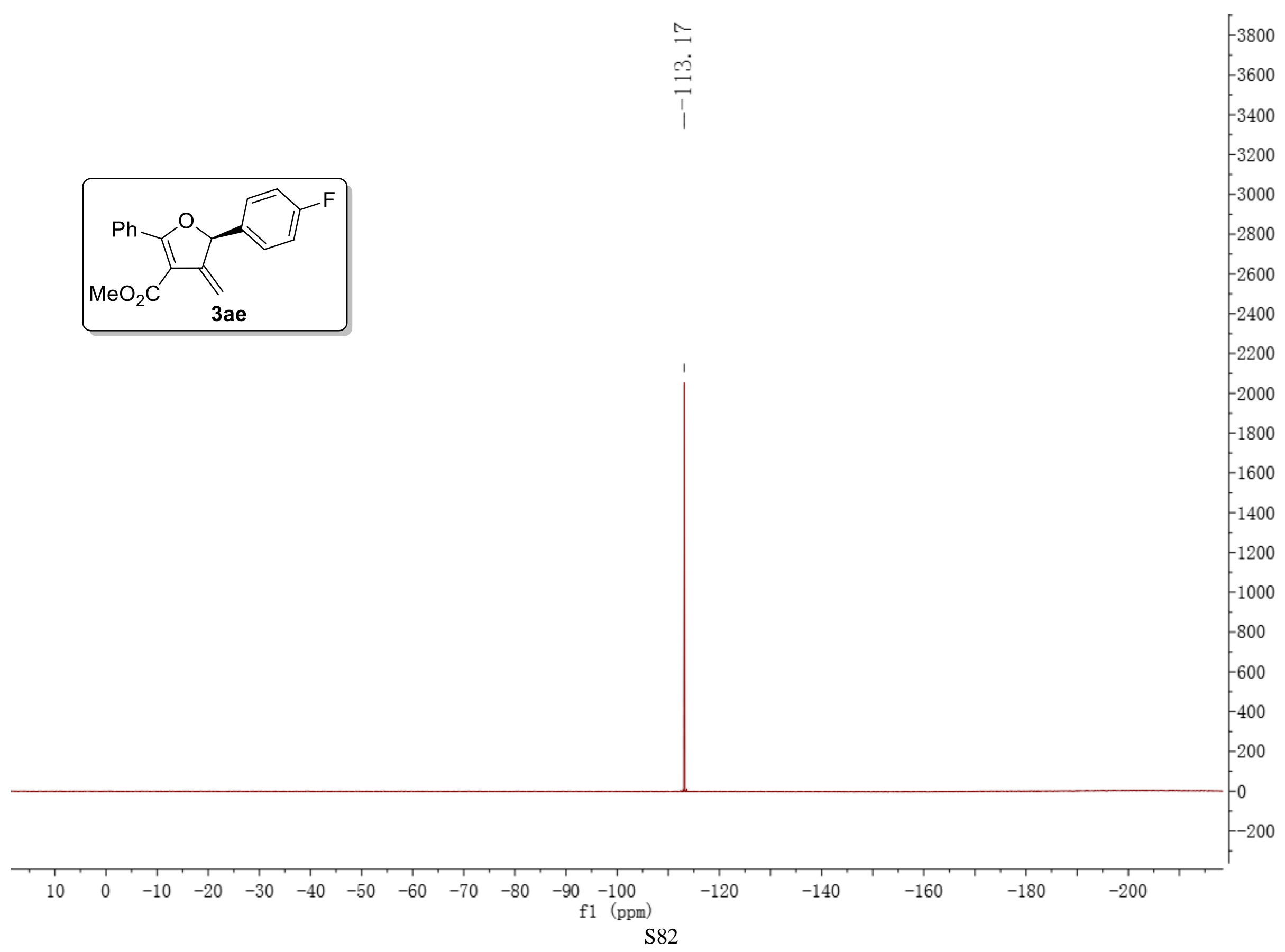




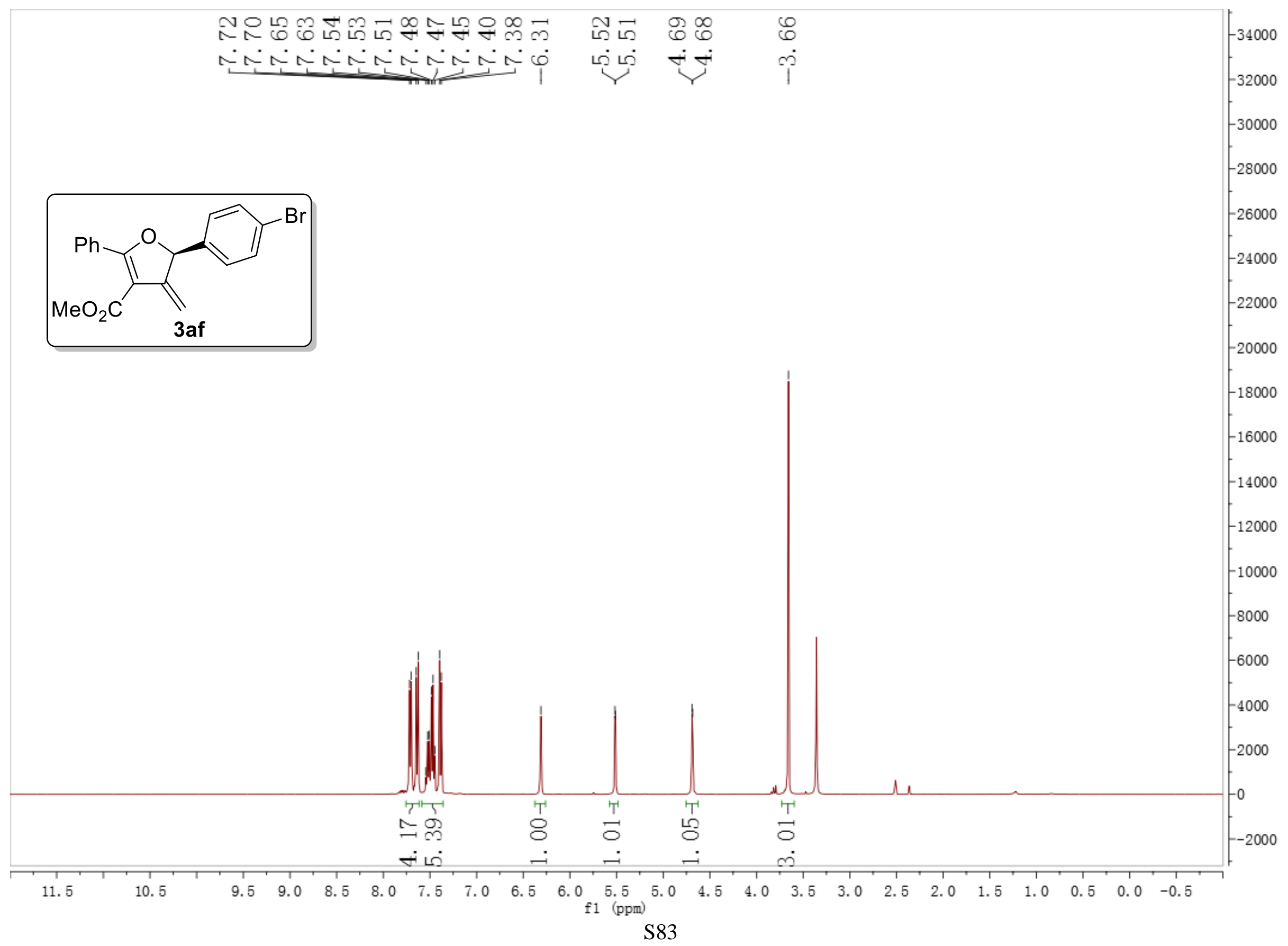




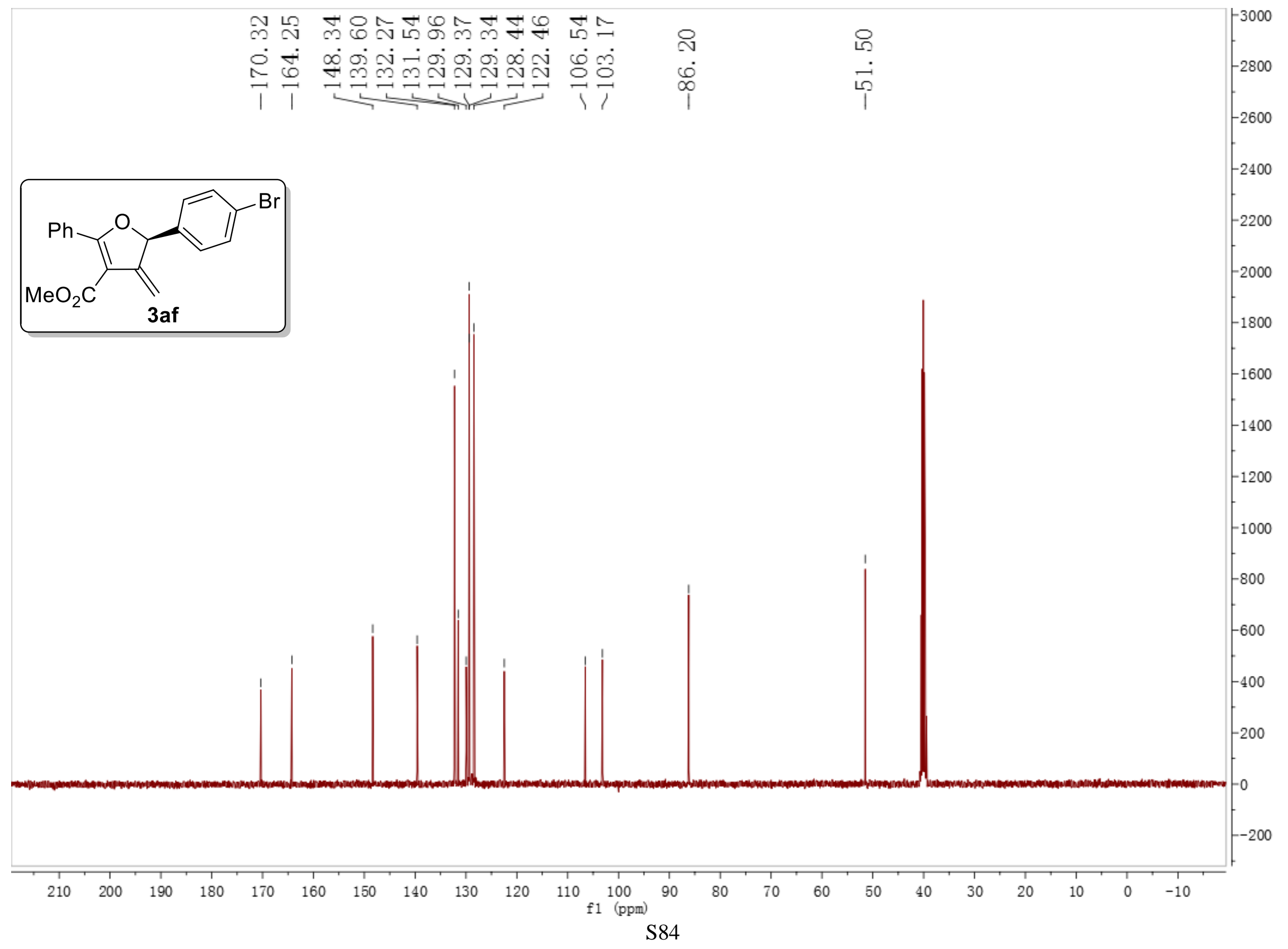




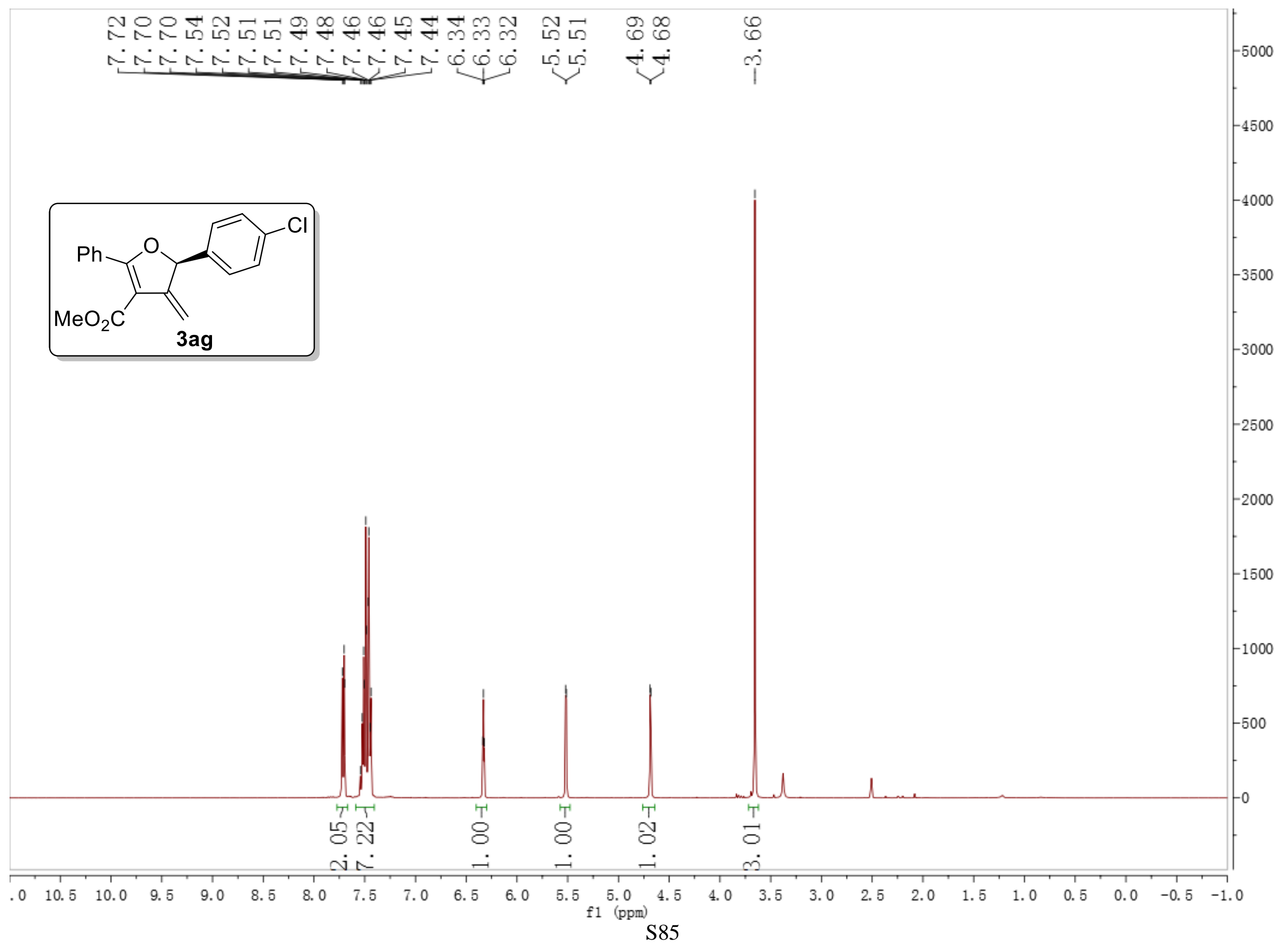




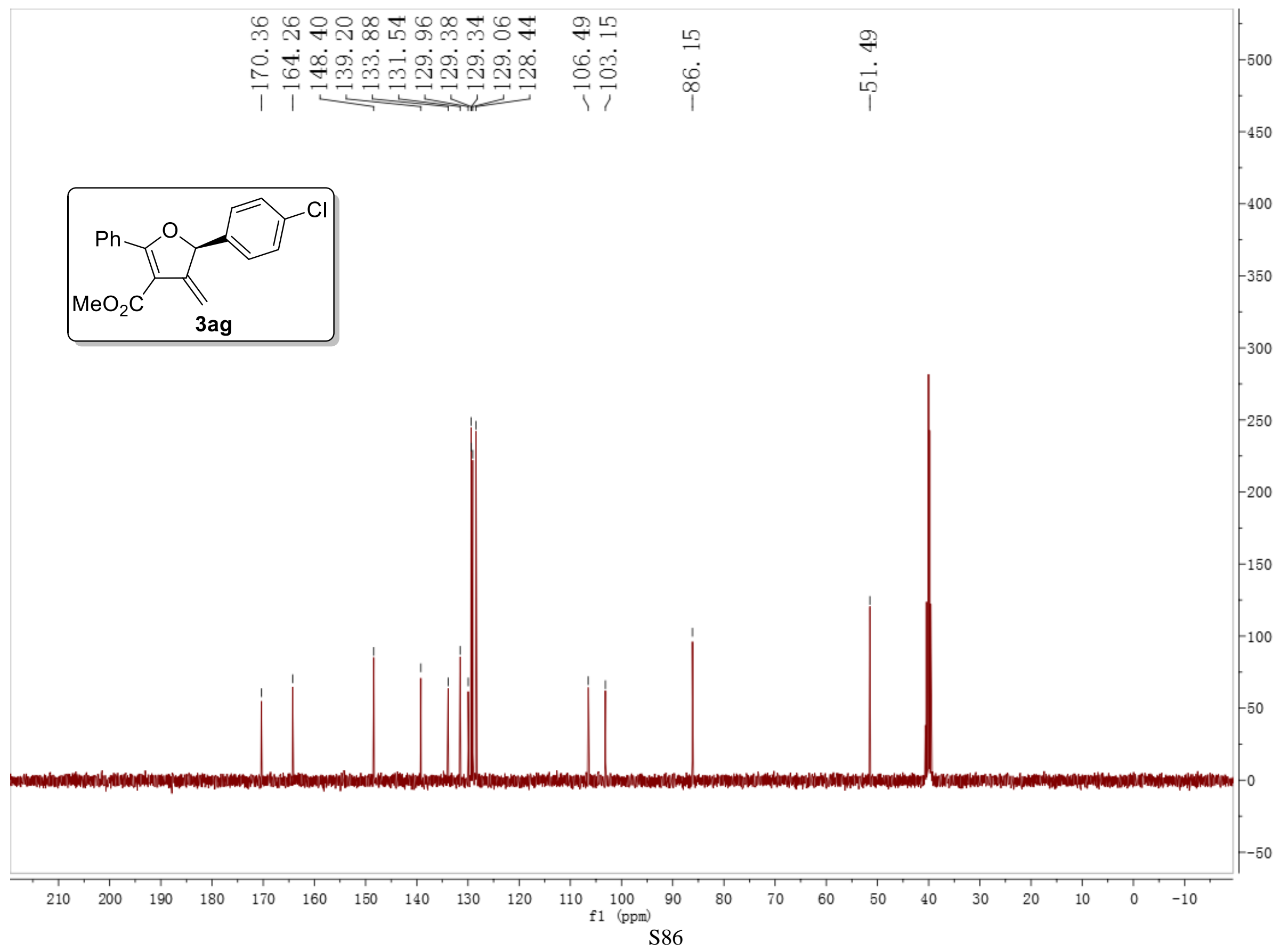




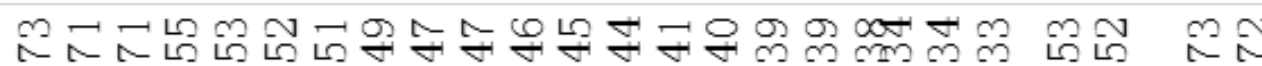

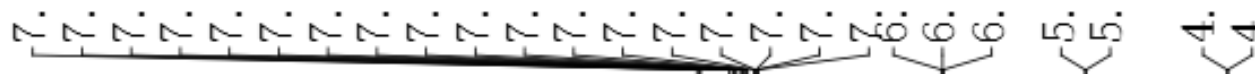
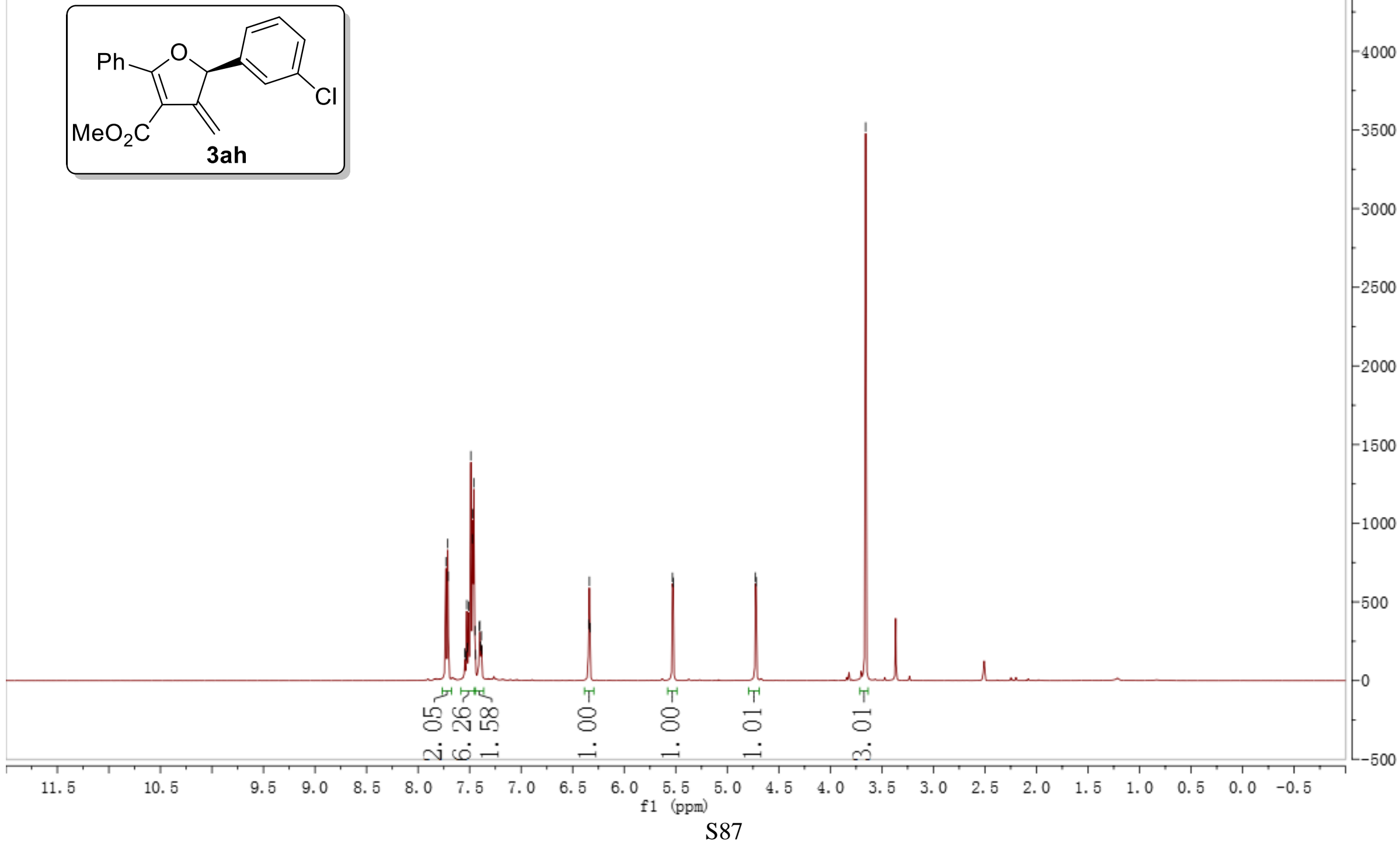


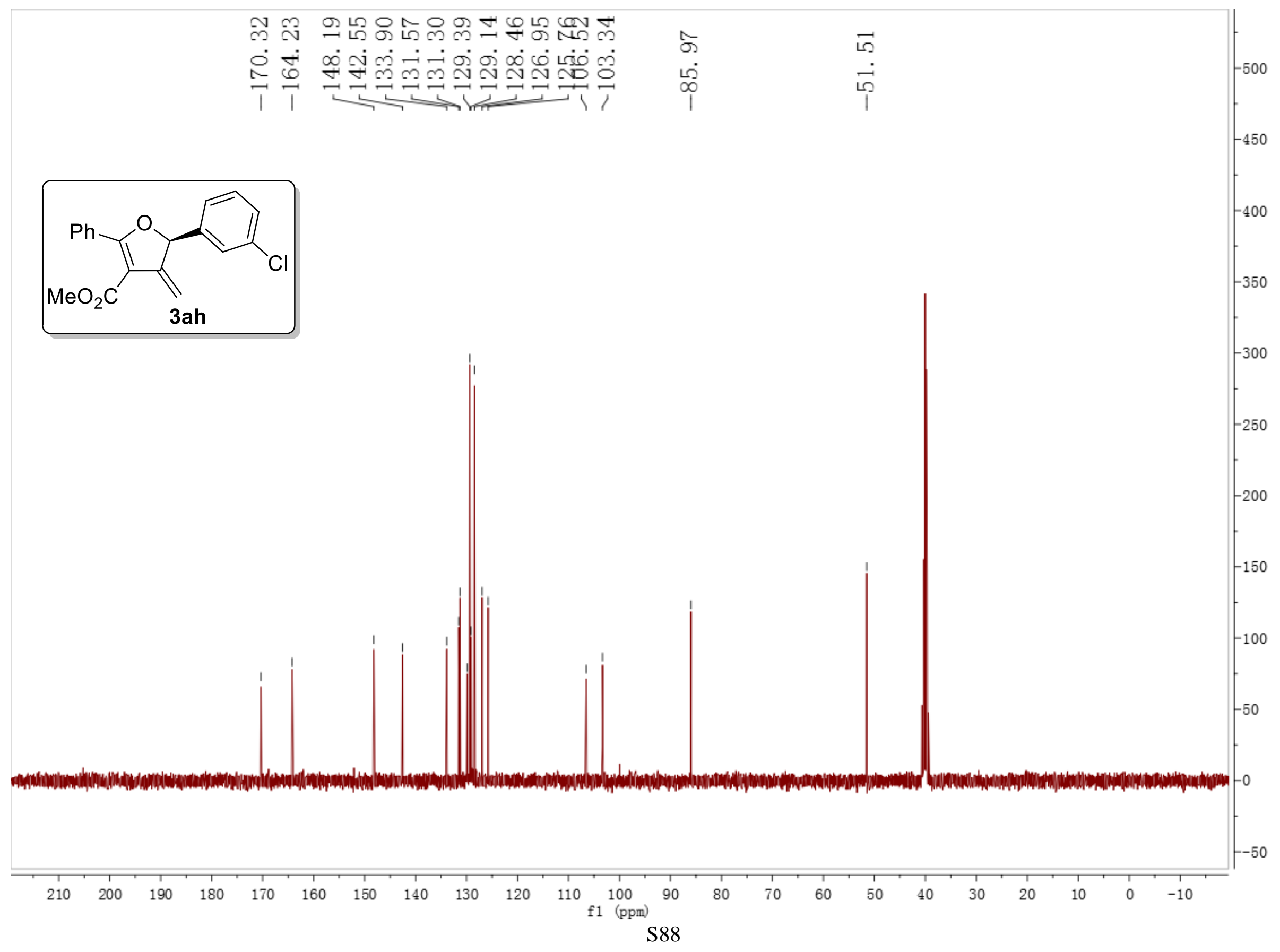




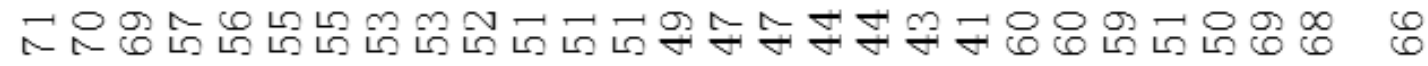

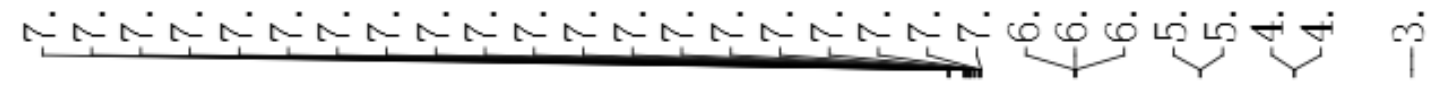
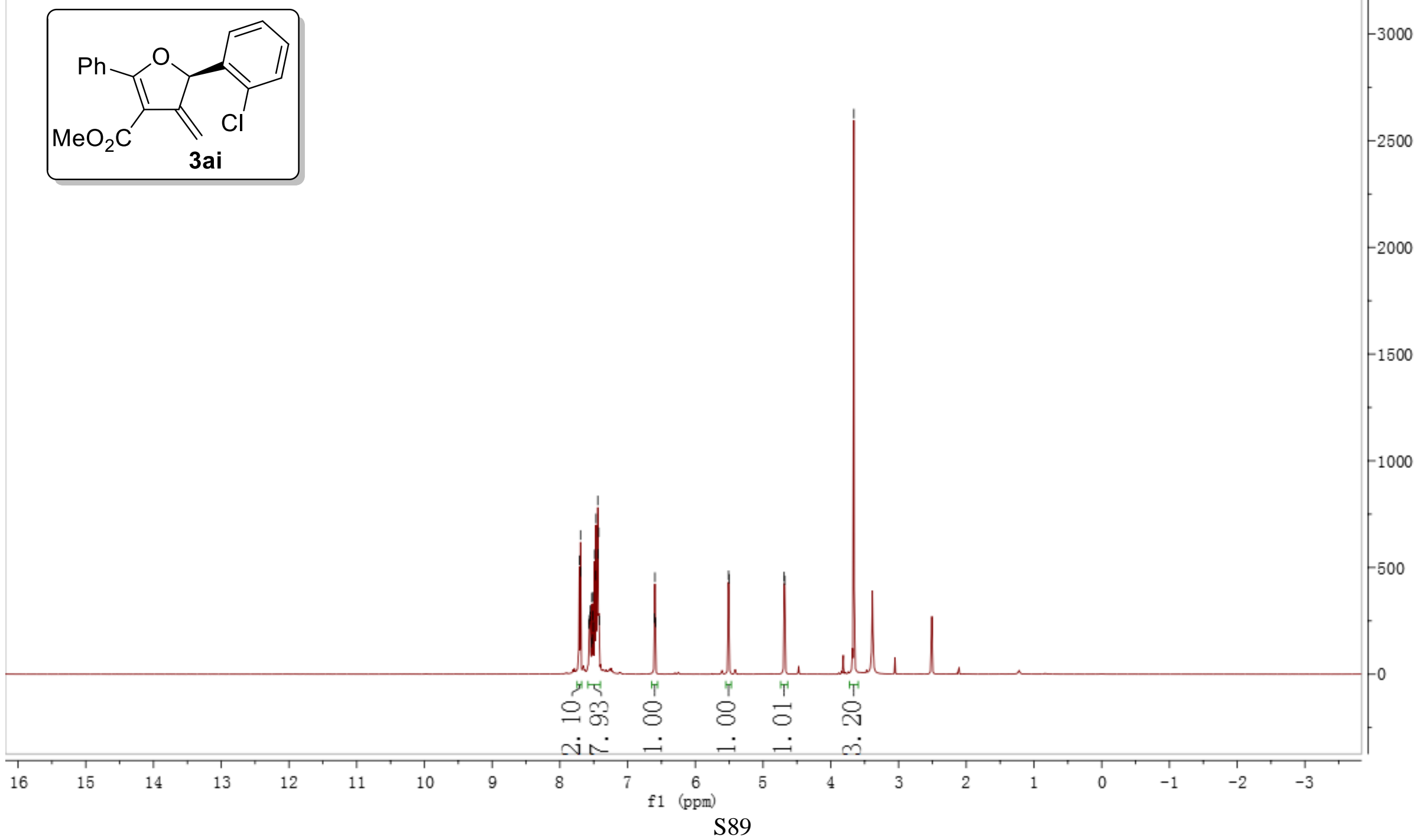


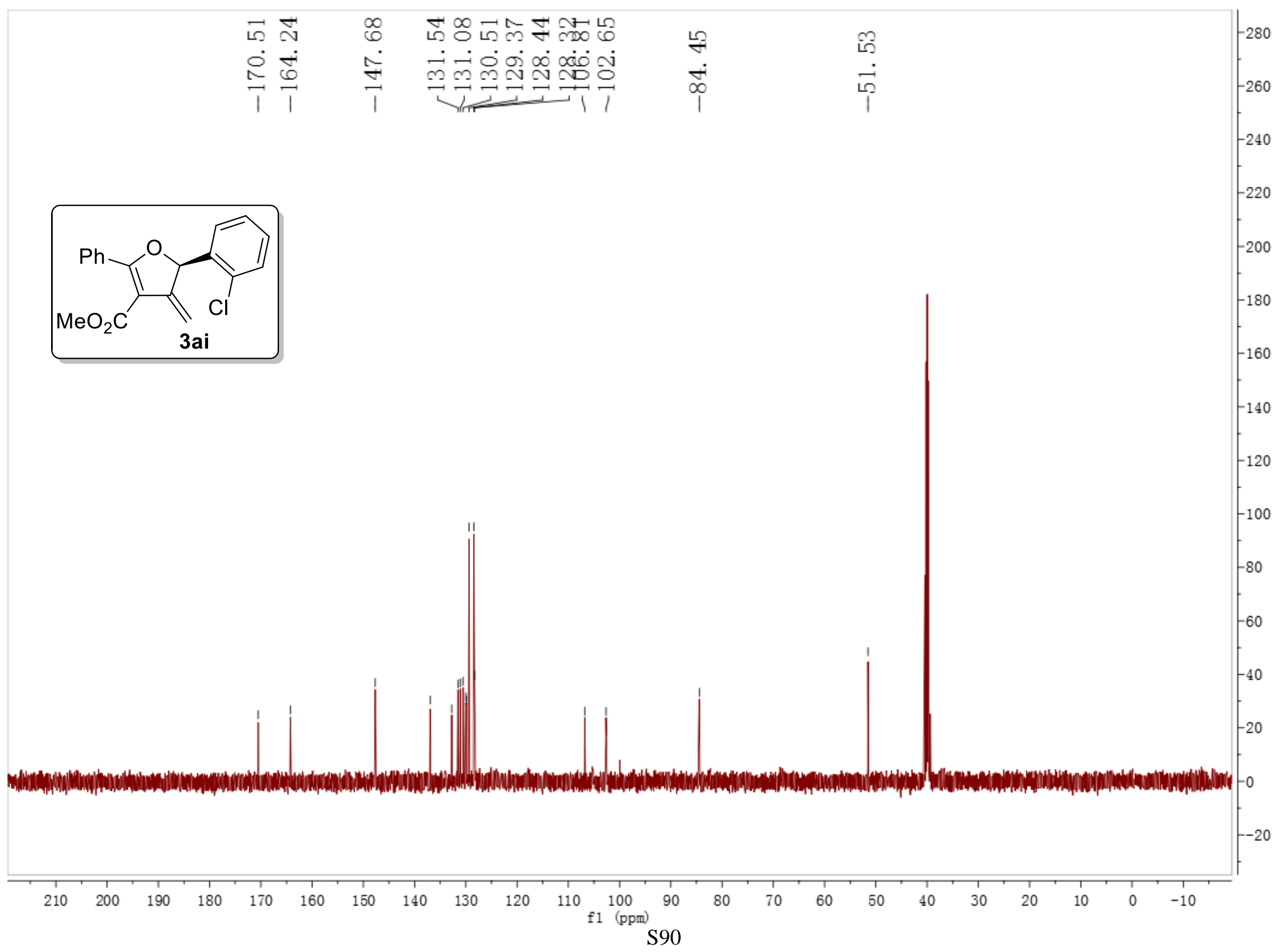




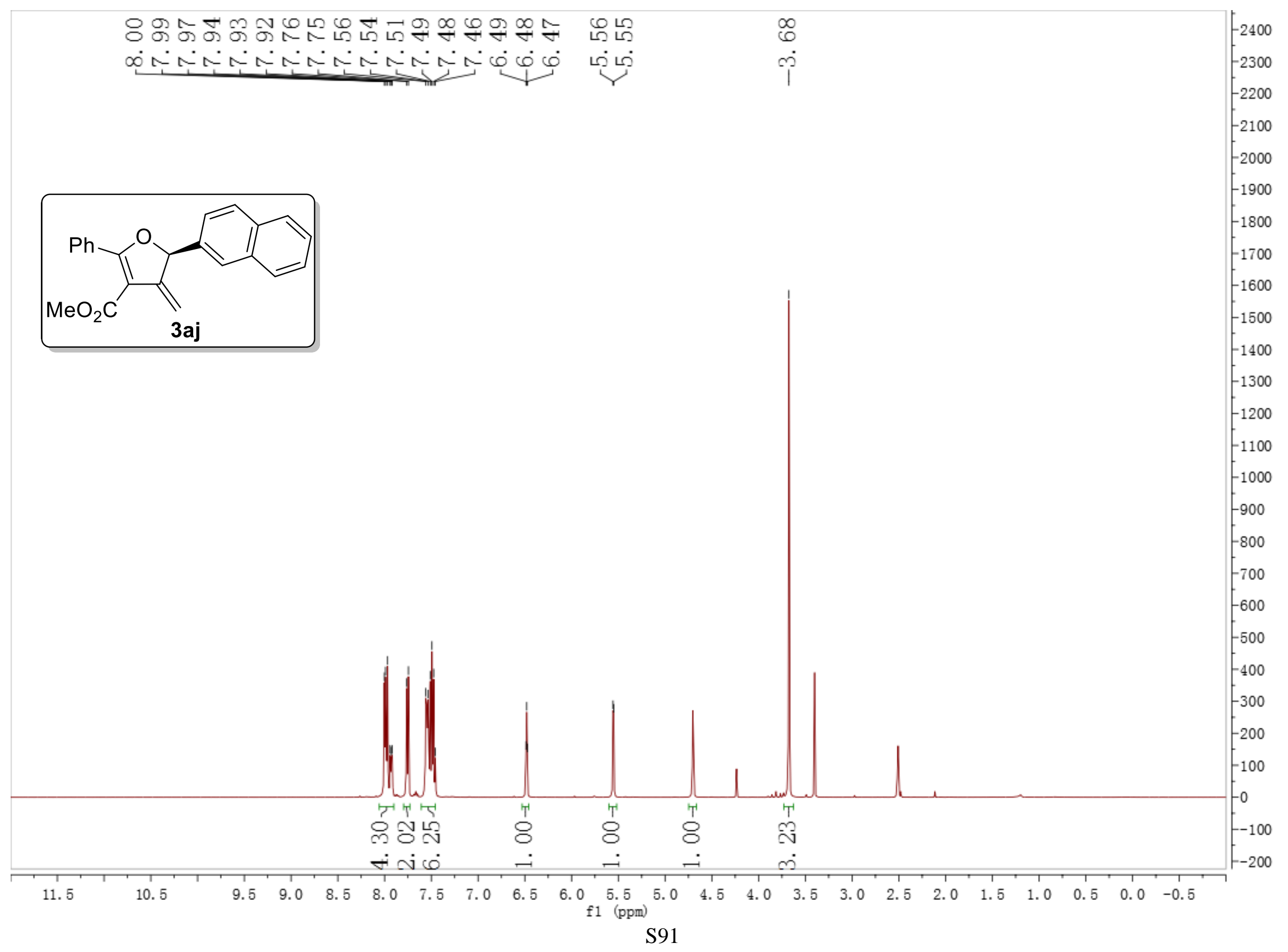




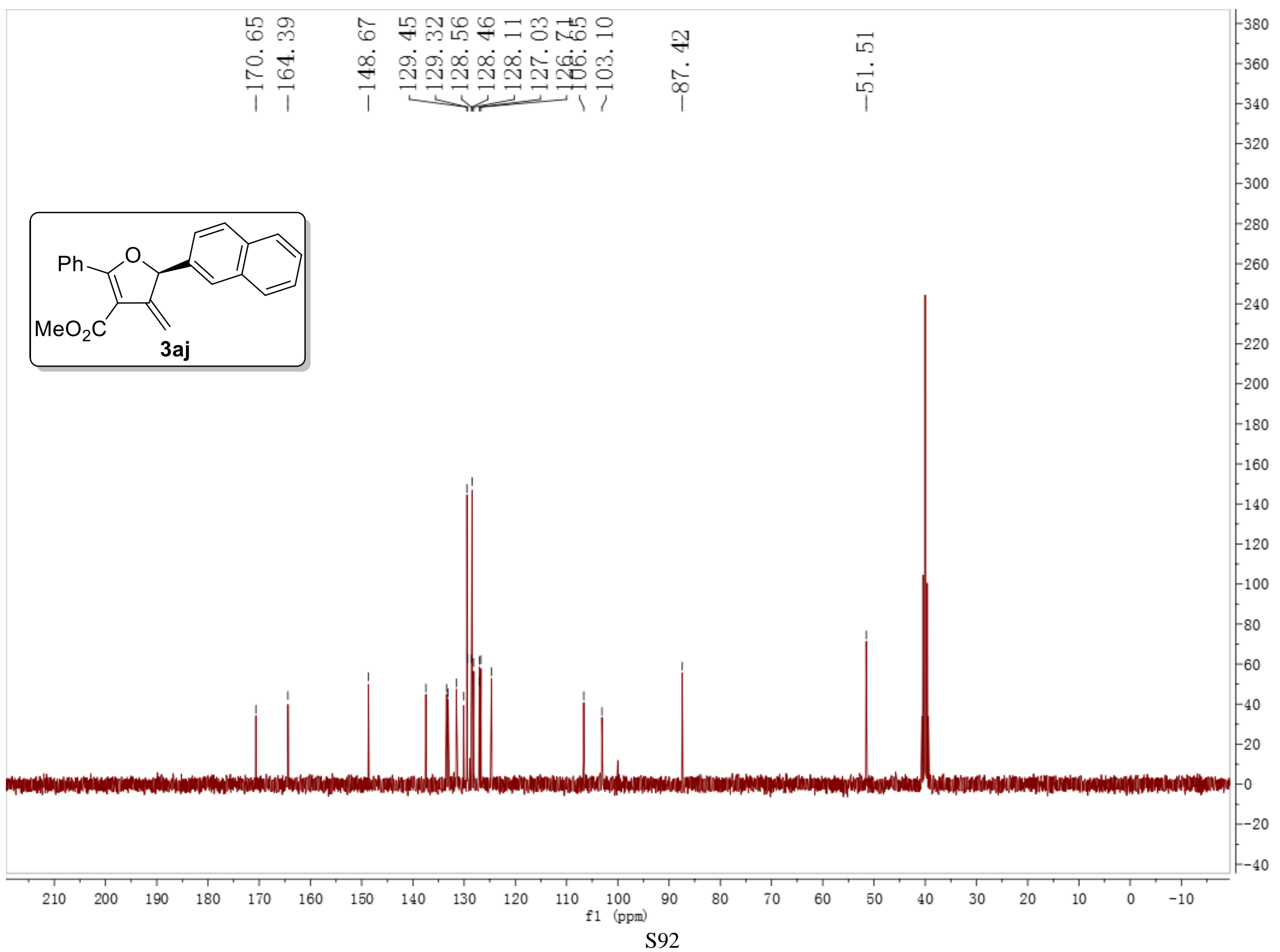




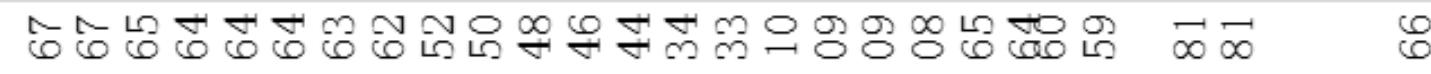

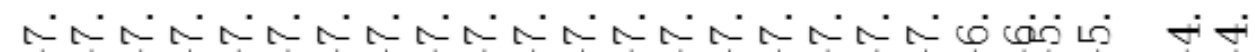

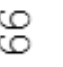

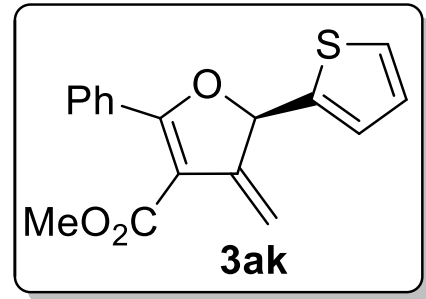

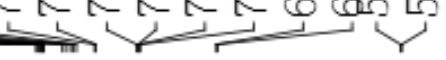

$\dot{+\infty}$

$-4500$

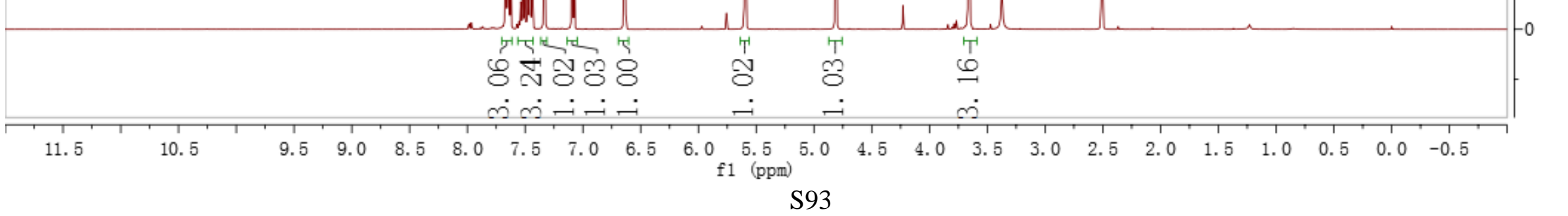




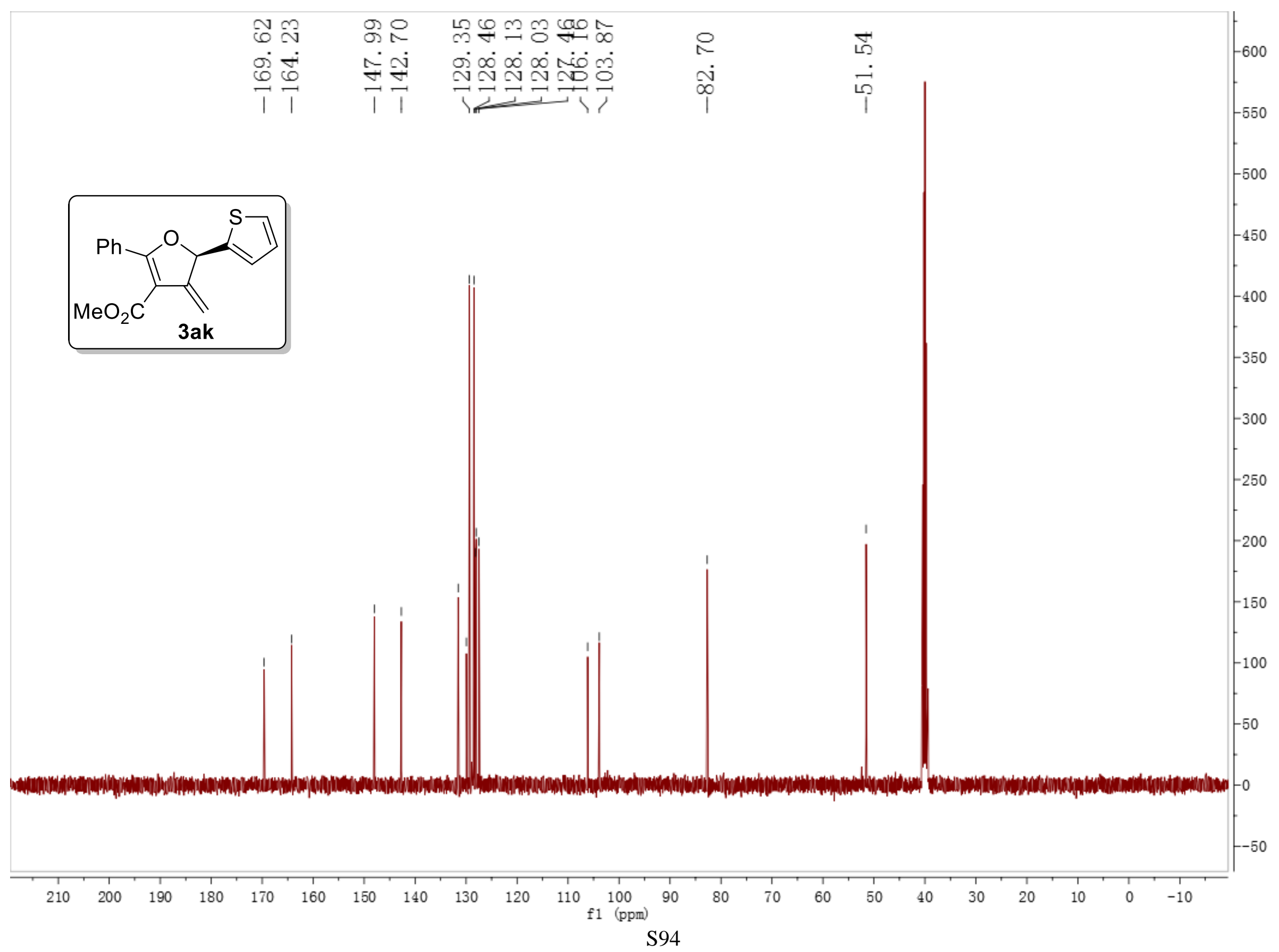




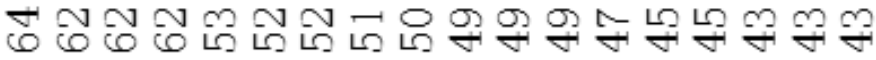

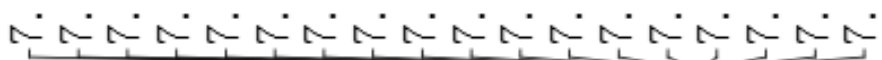

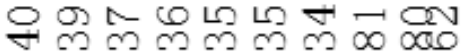

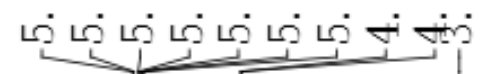
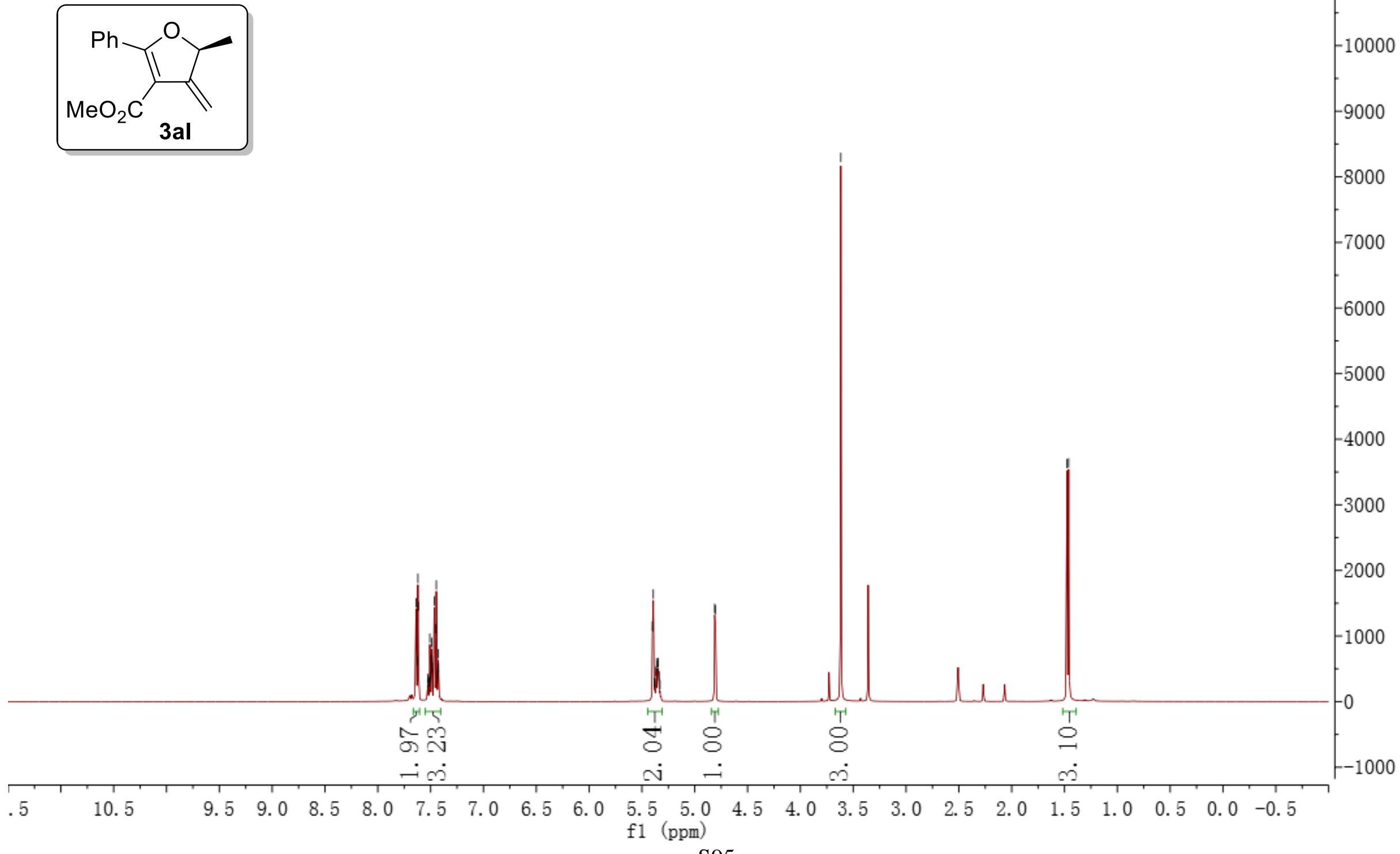


\begin{tabular}{|c|c|c|c|}
\hline & $\stackrel{2}{2}$ & 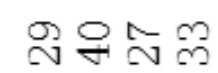 & $\exists \stackrel{\sim}{\sim}$ \\
\hline$\dot{;}$ & 0 & 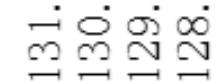 & $\dot{8}$ \\
\hline
\end{tabular}

$\overrightarrow{\vec{m}}$

$\stackrel{0}{\mathrm{~N}}$
$\stackrel{\mathrm{j}}{\mathrm{j}}$
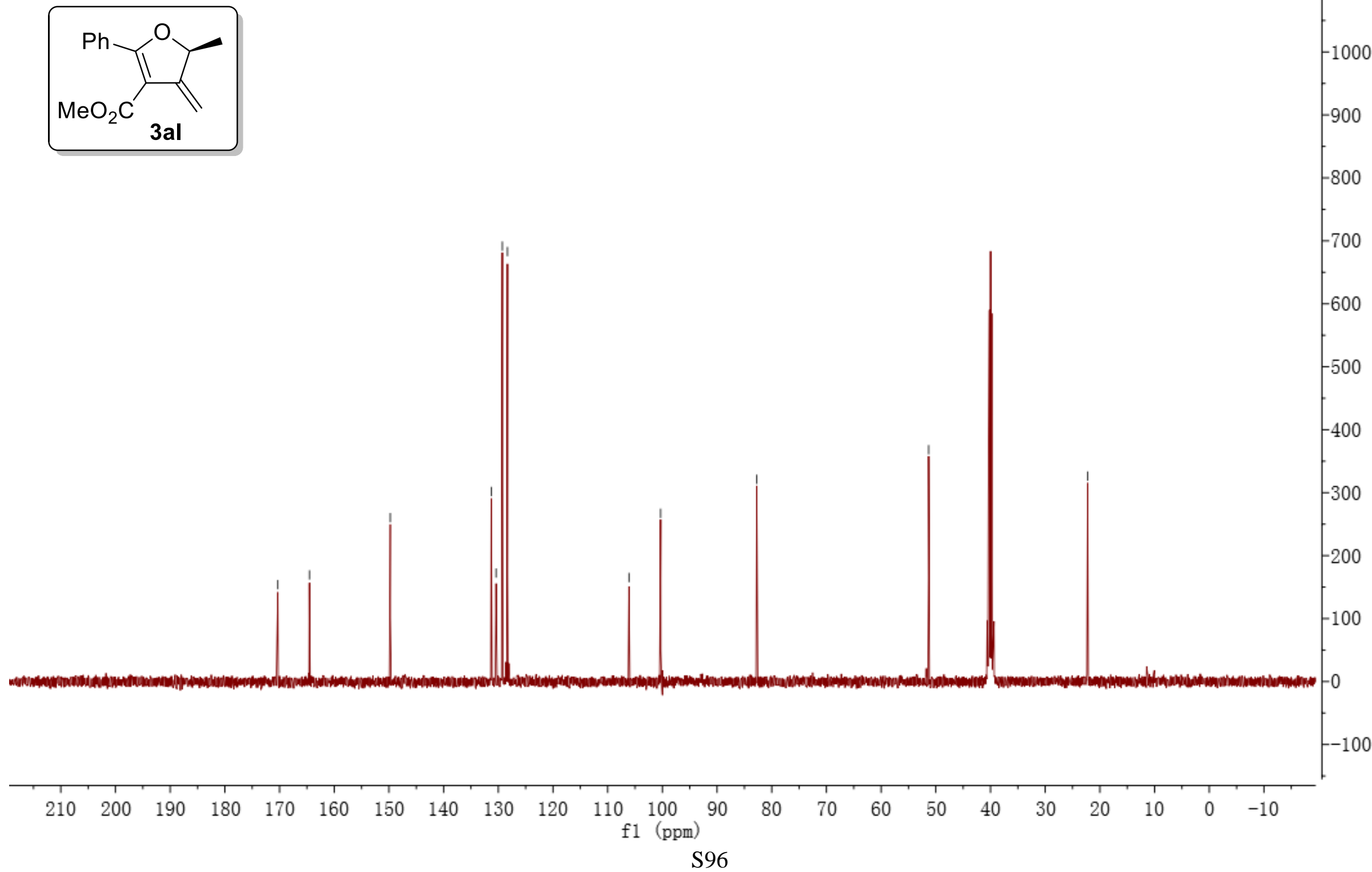


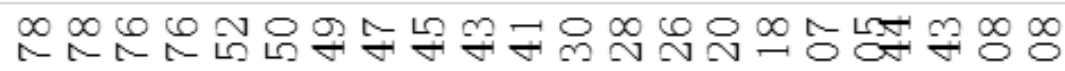

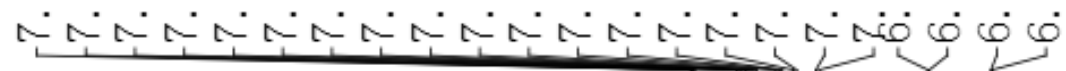

$\vec{p}$

$-2800$
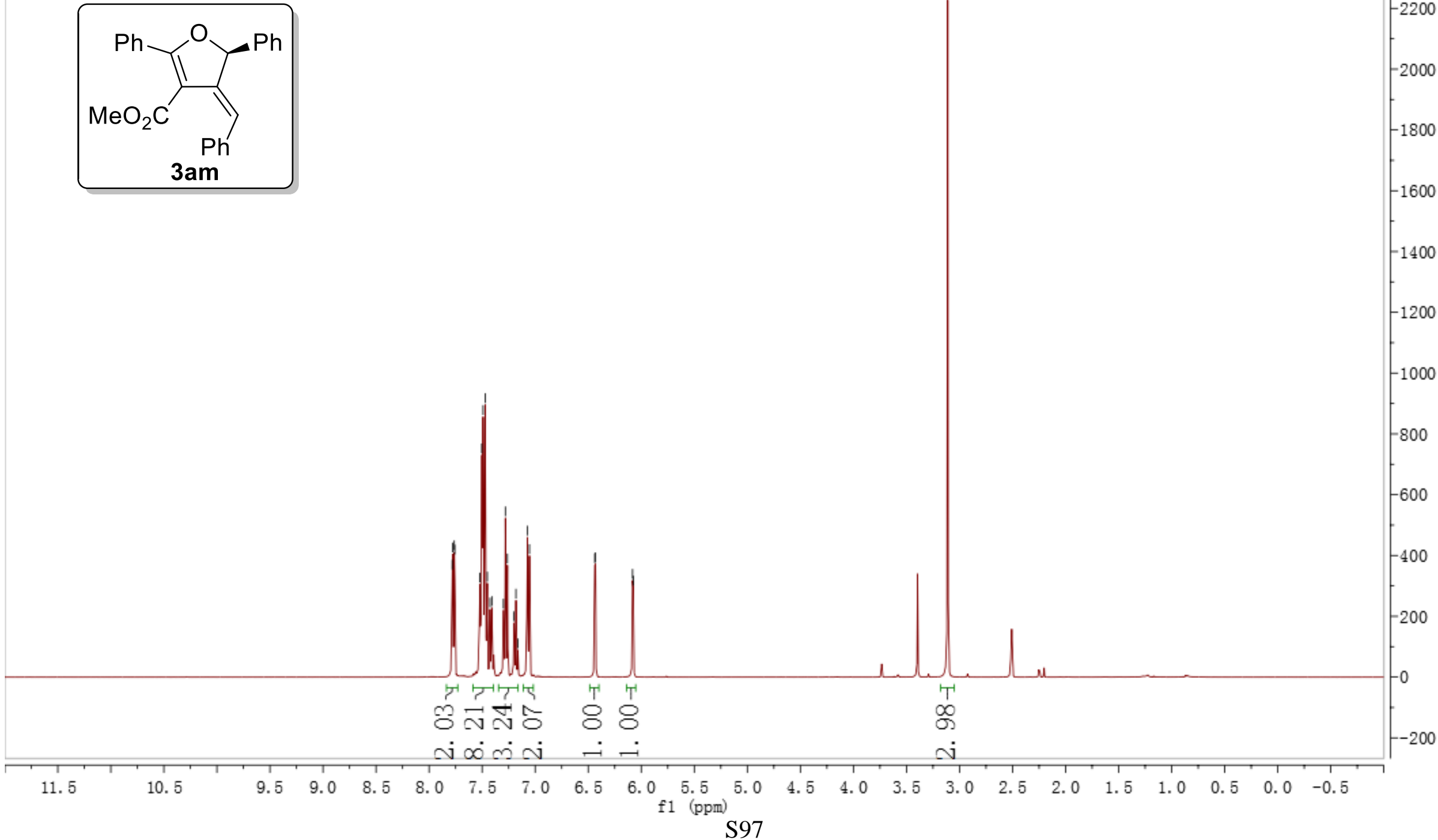


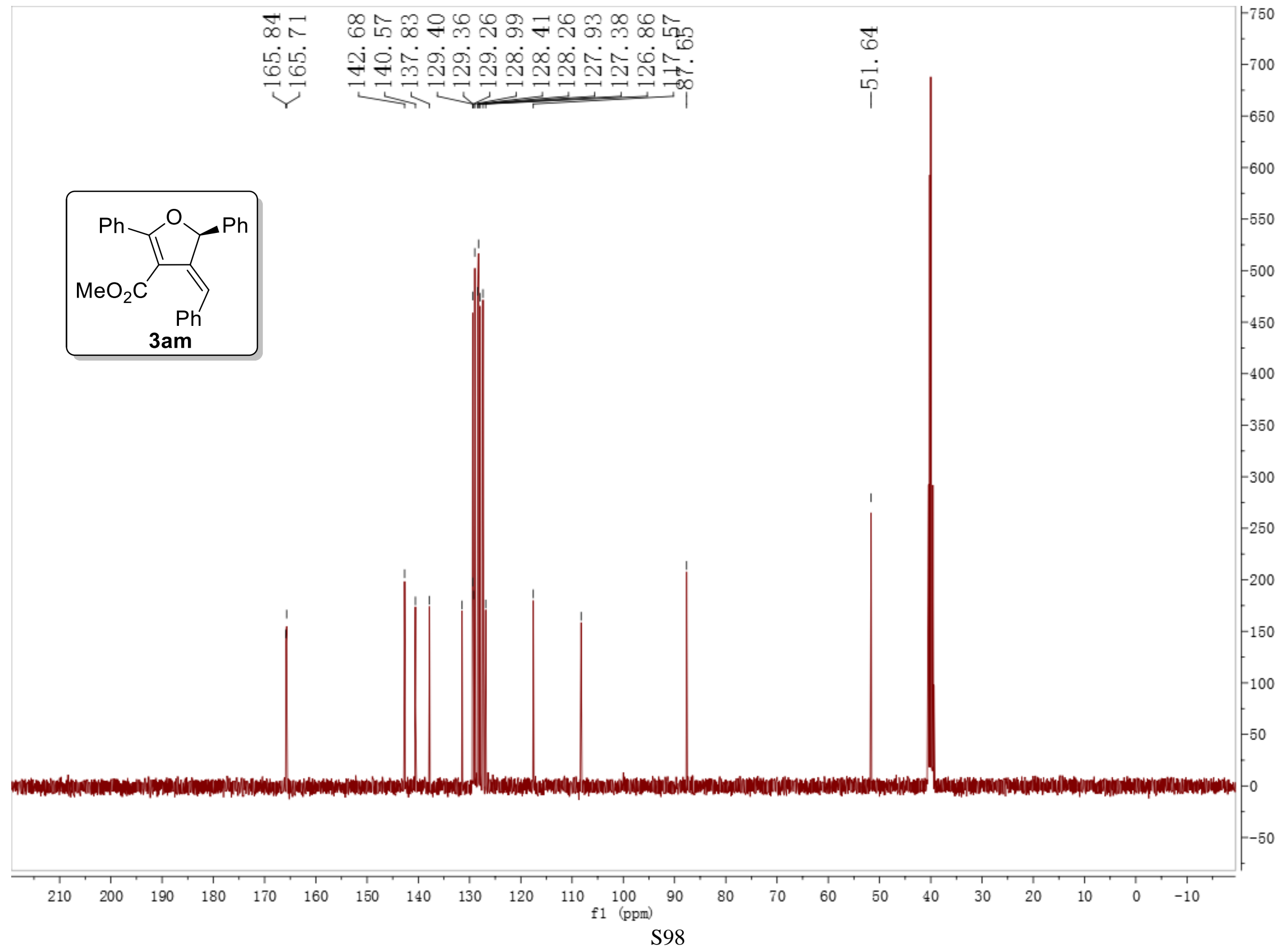




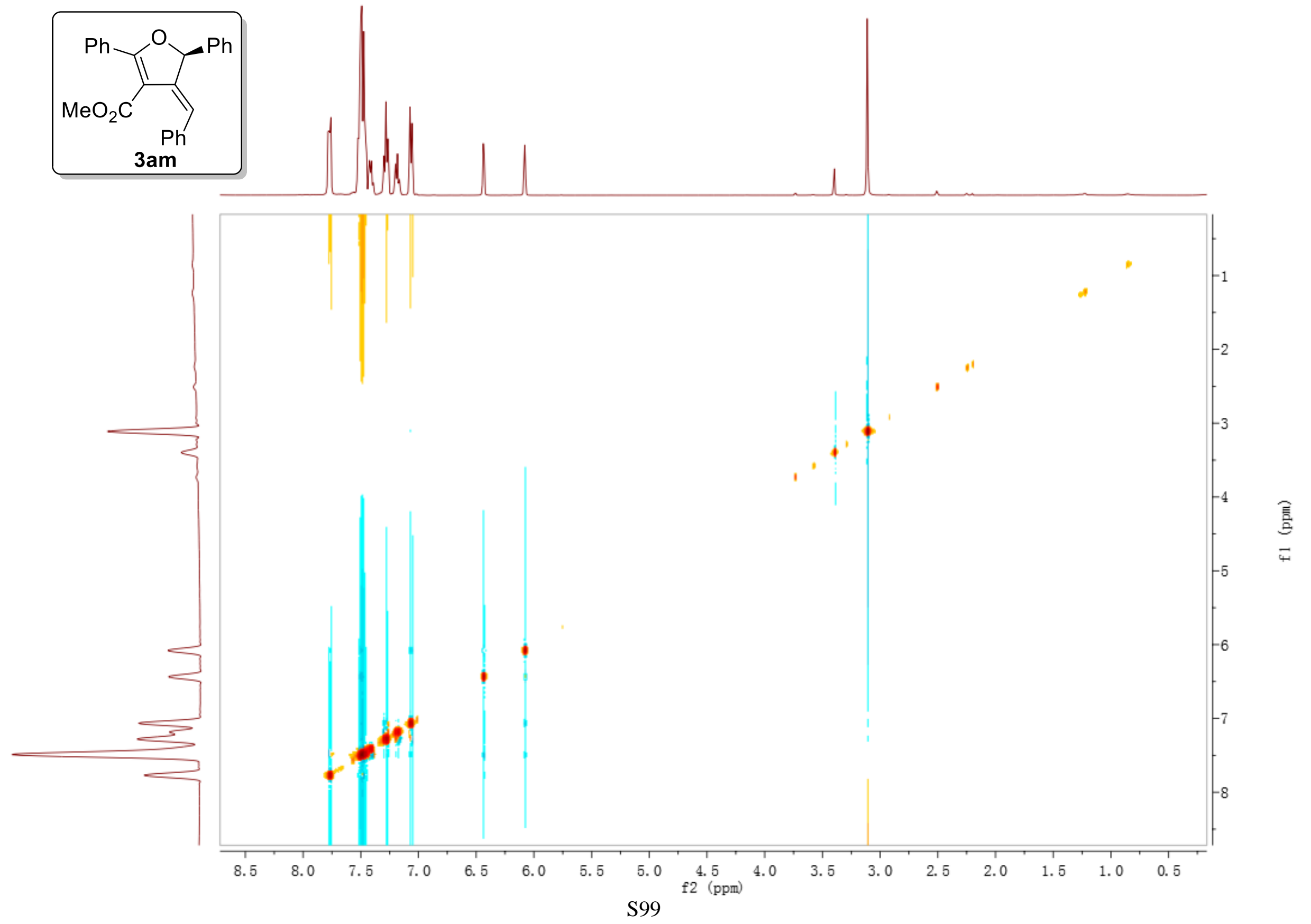




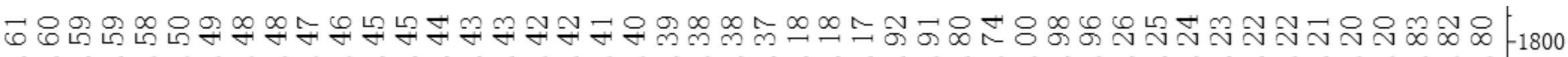

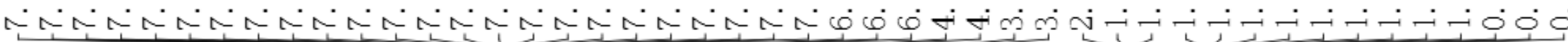
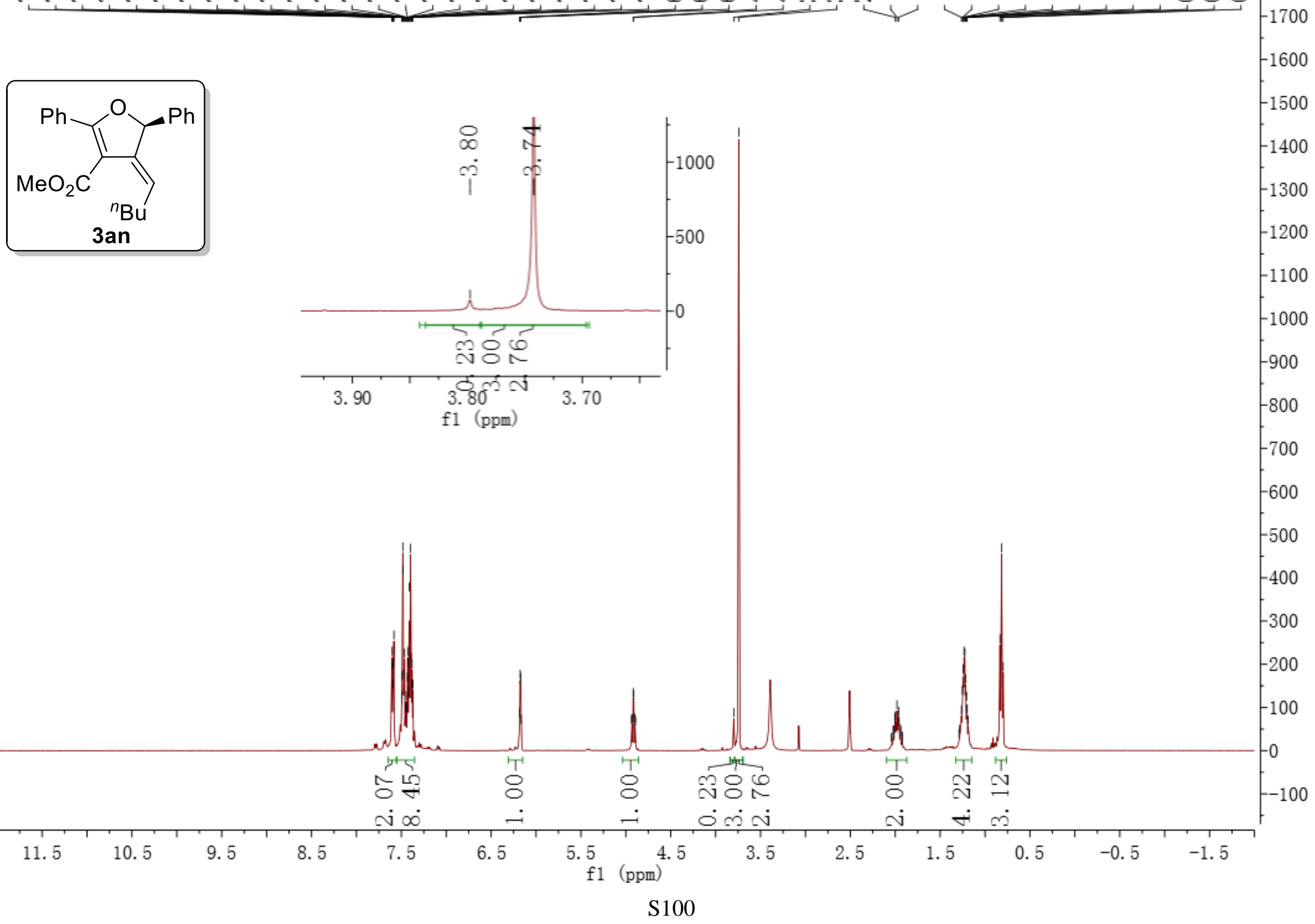


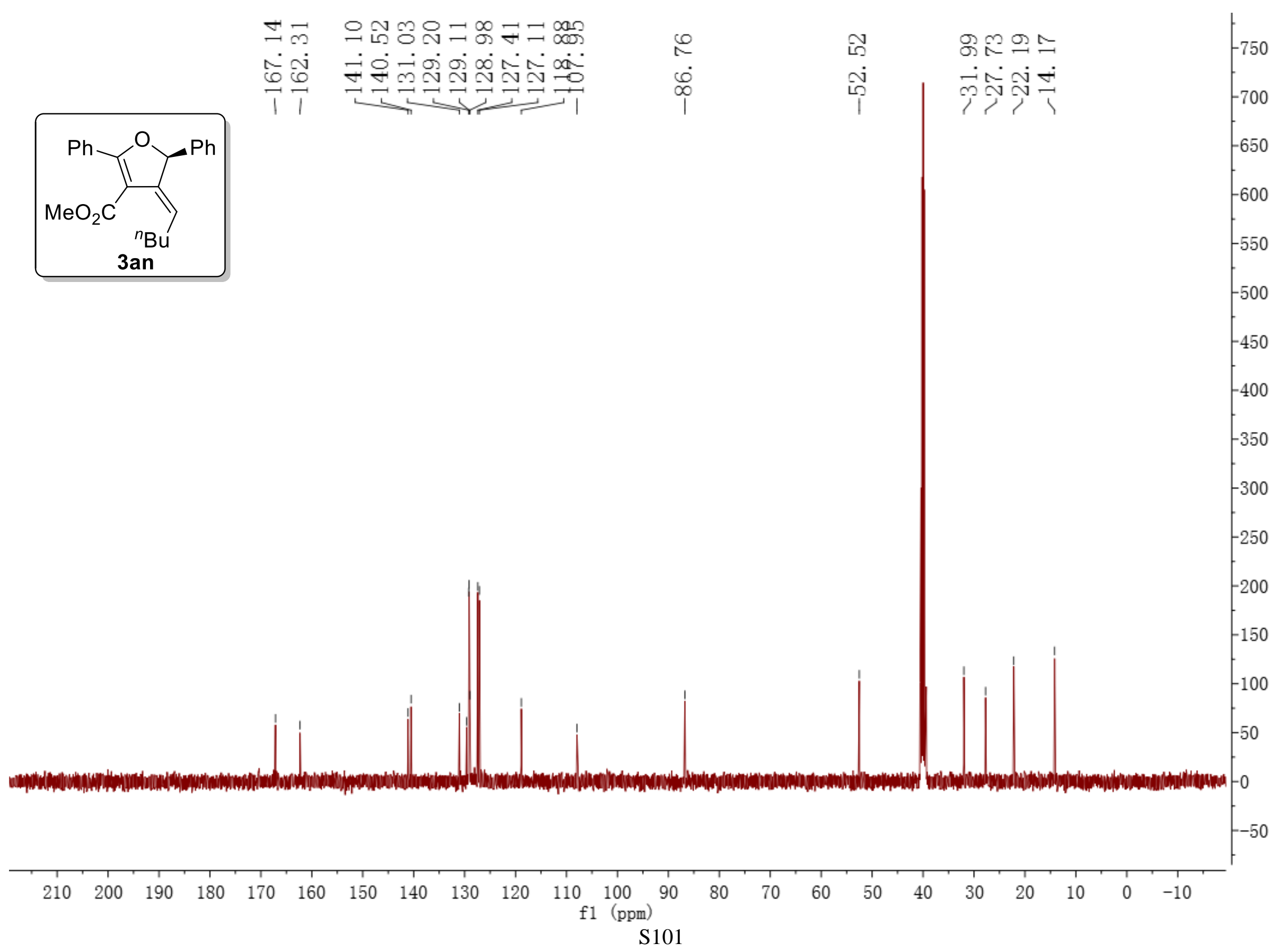




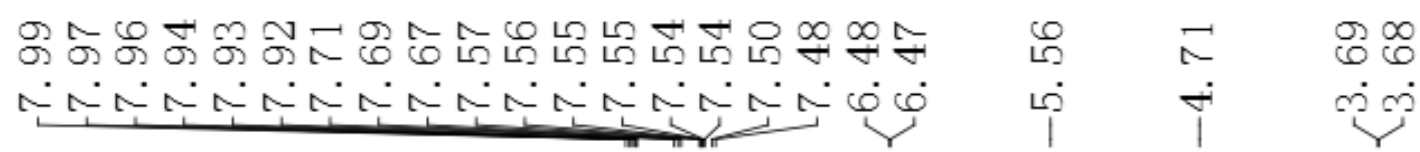
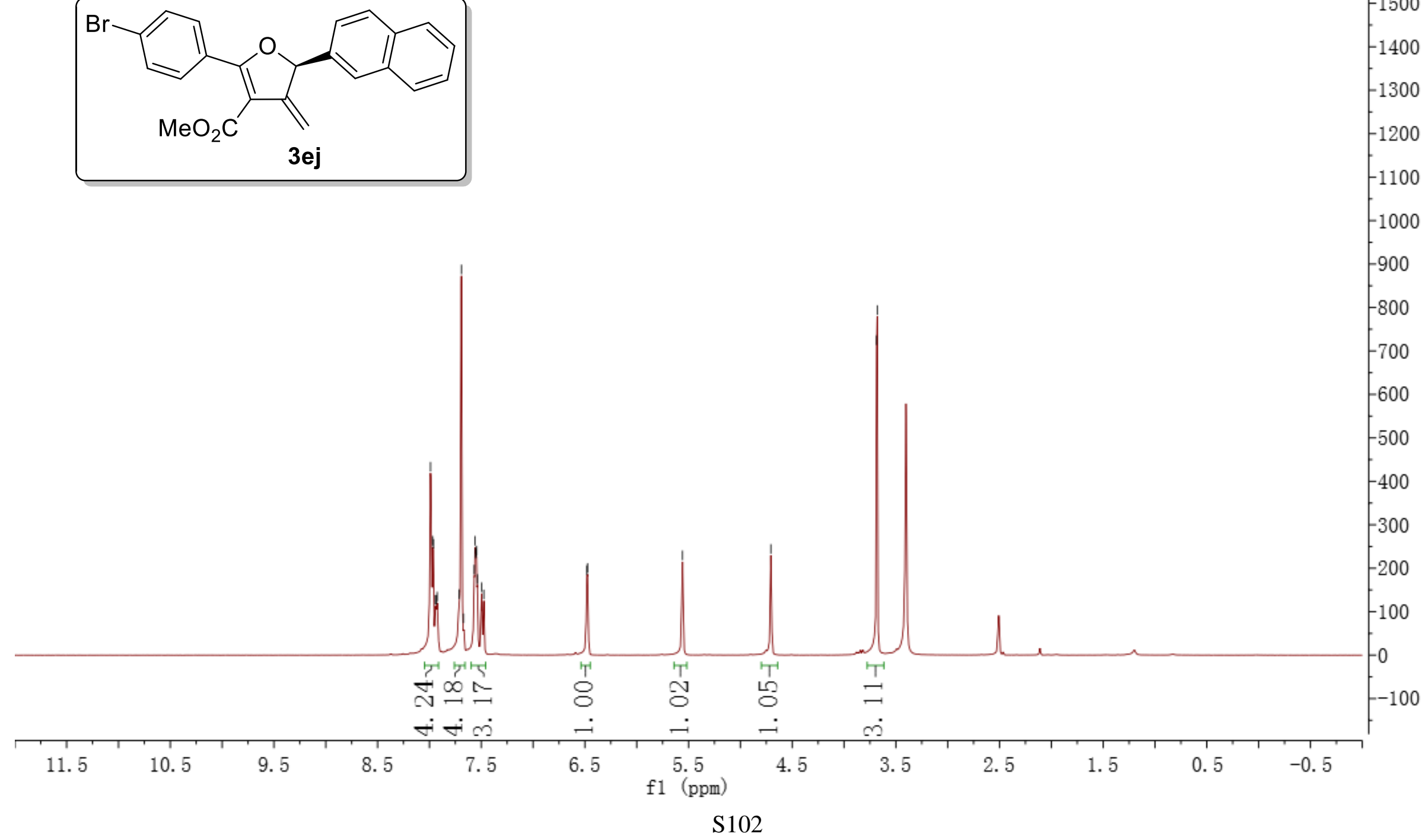


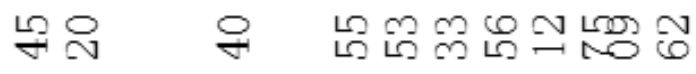

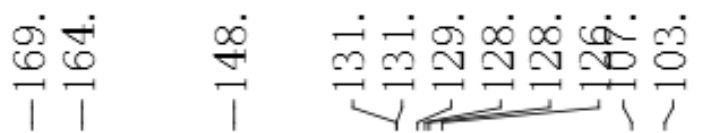
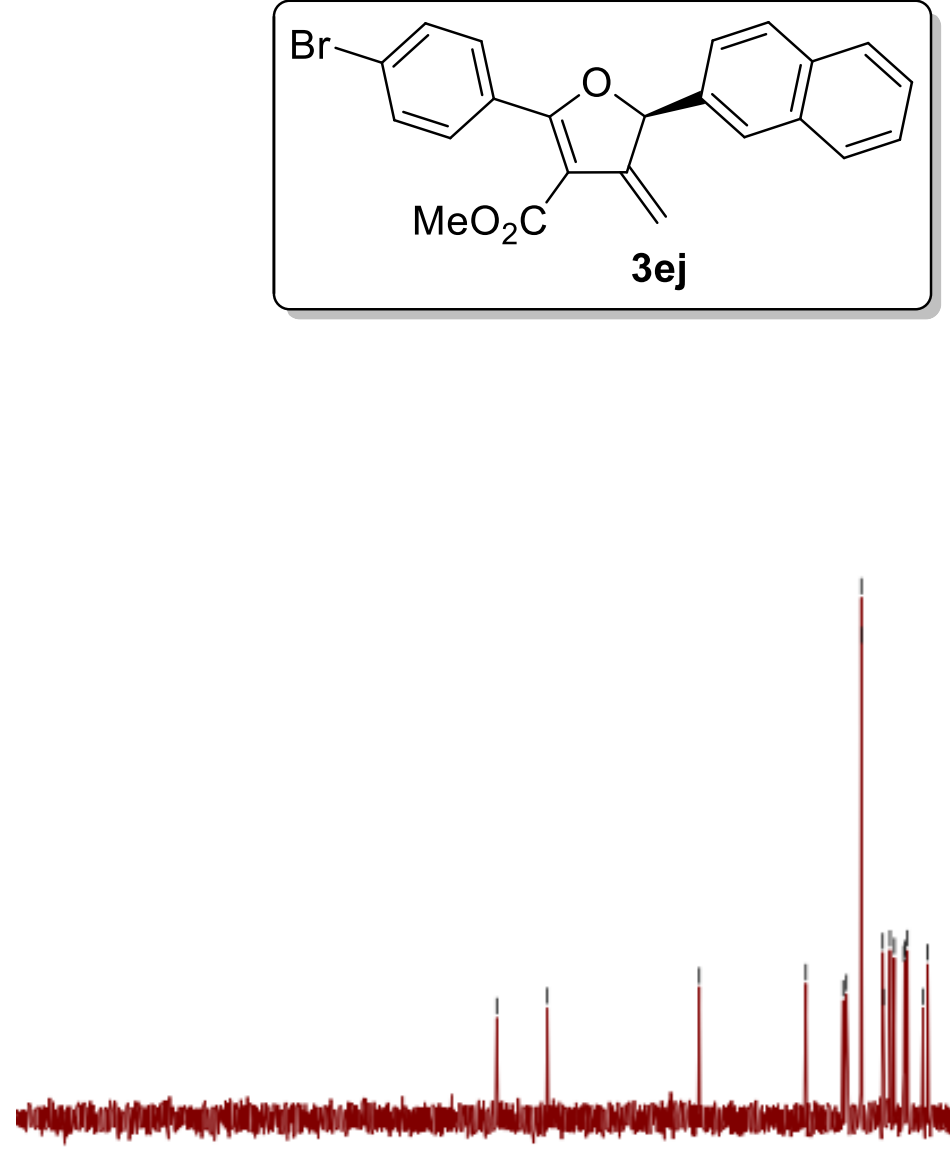


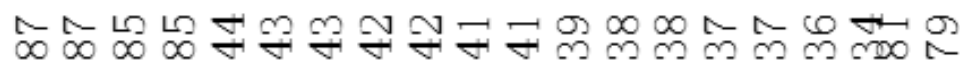

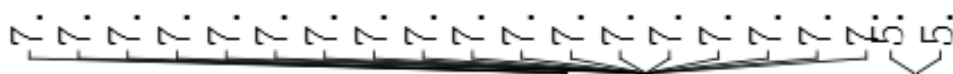

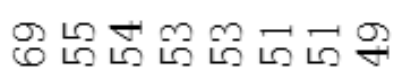

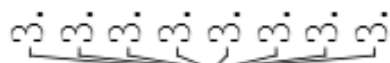
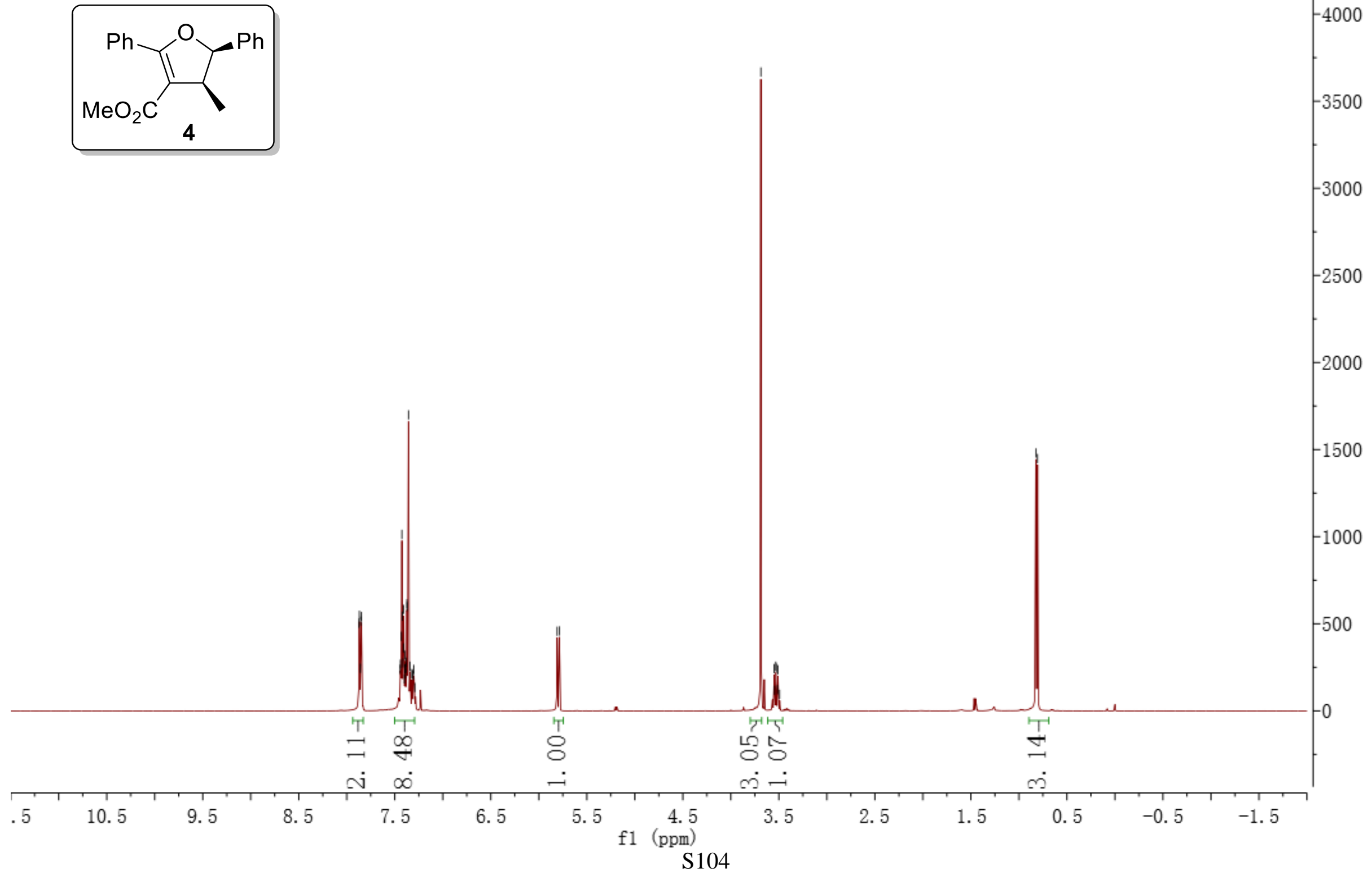

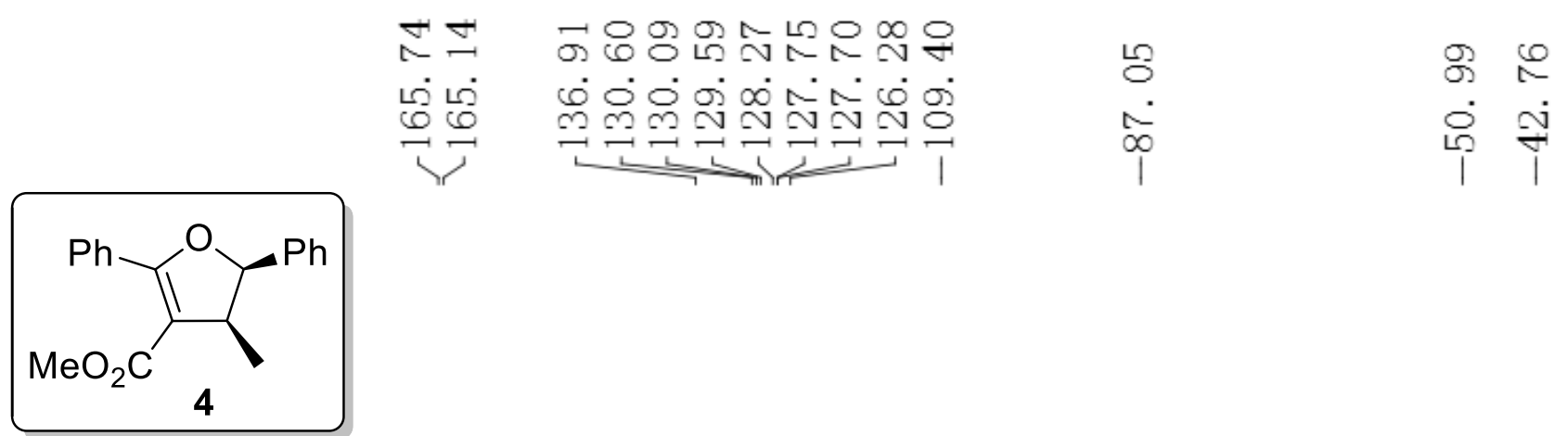

के

r 1 UU 


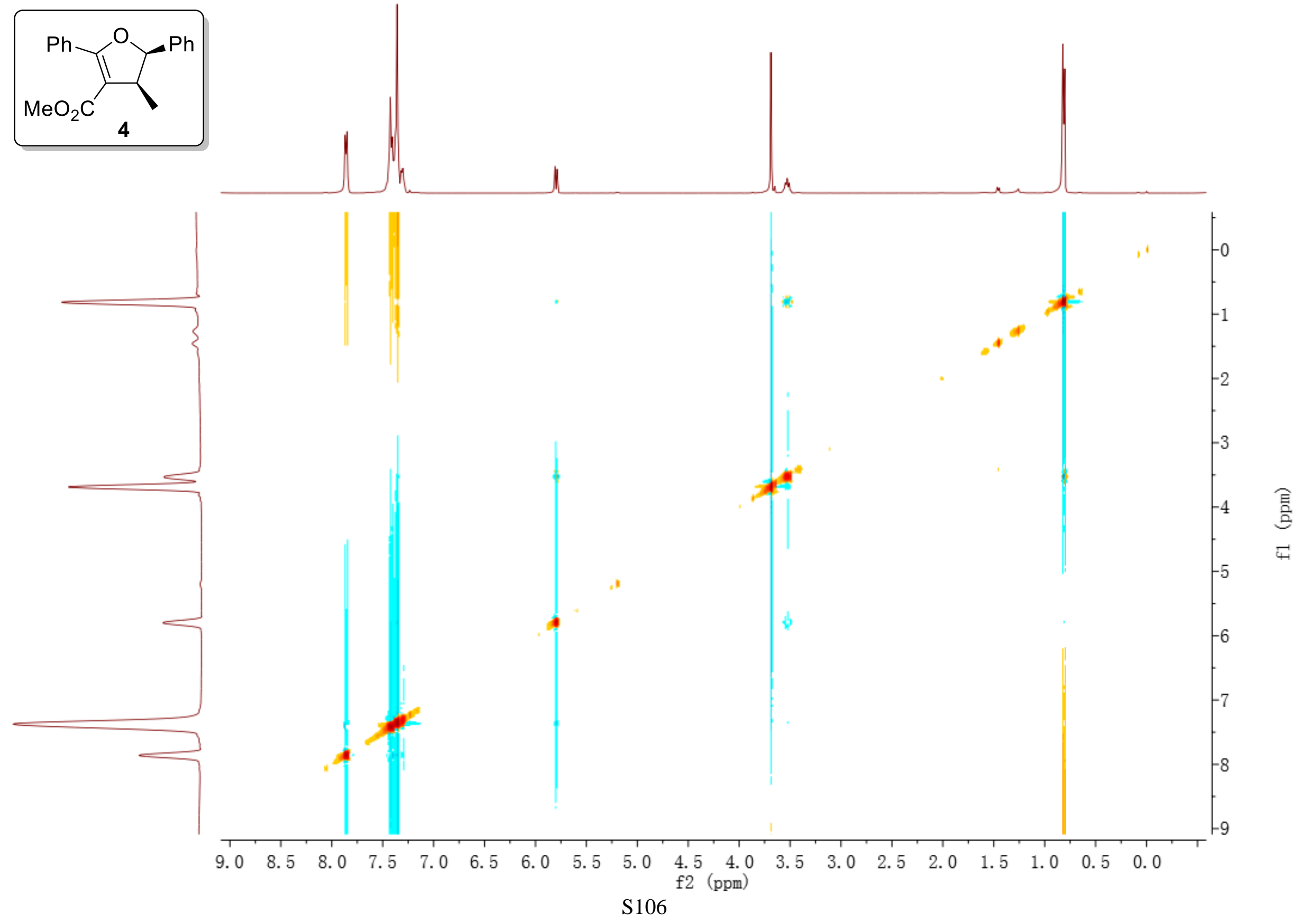




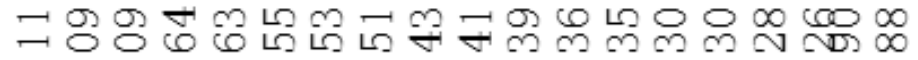

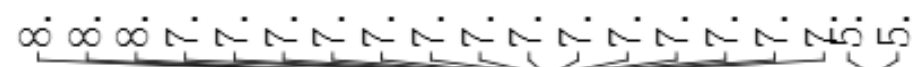

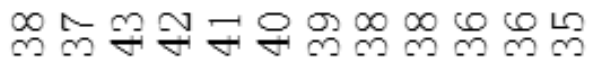

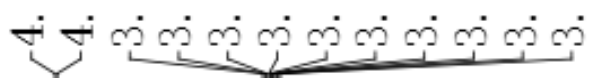

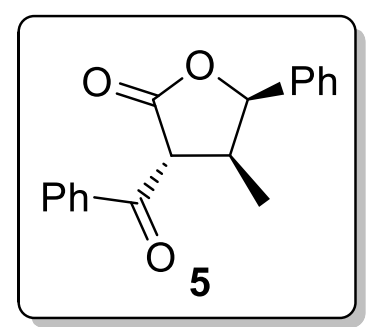

$\stackrel{\infty}{\infty} \infty$

சं

$-2100$

$-2000$

$-1900$

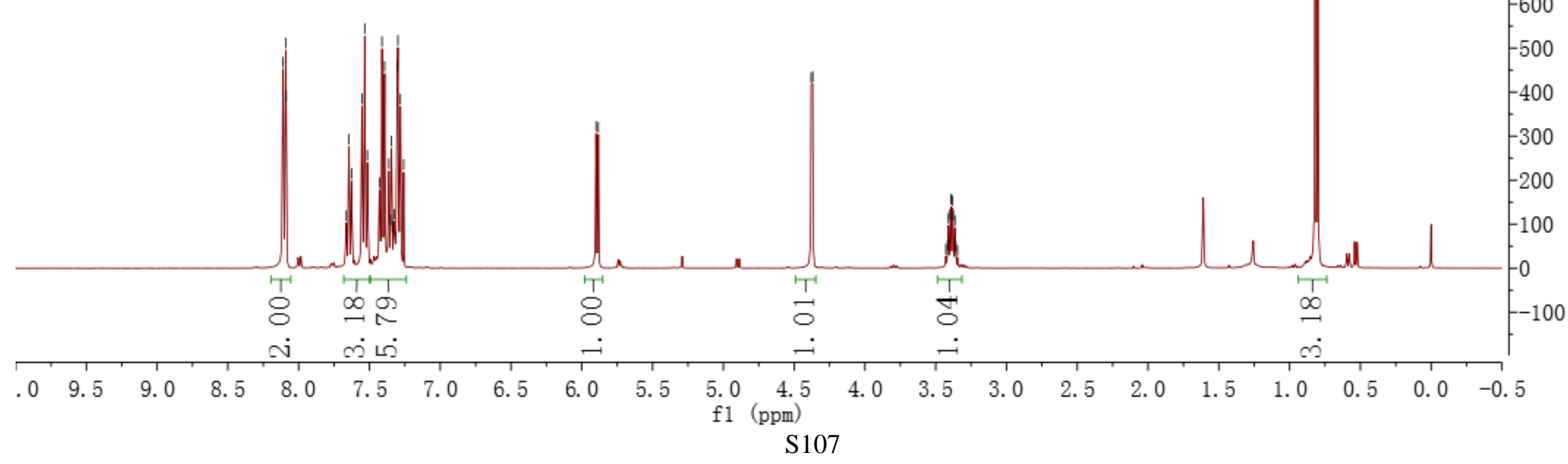




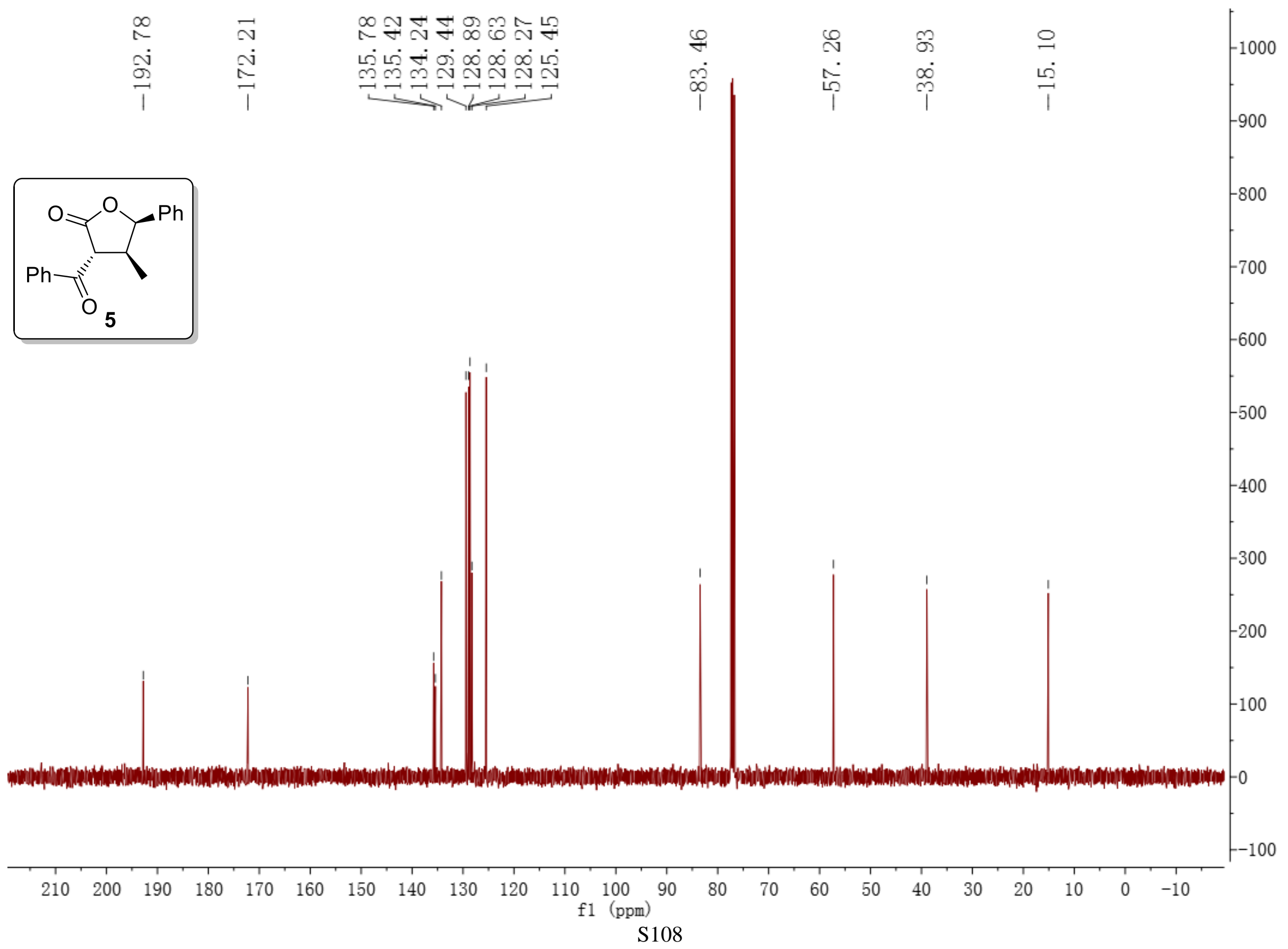




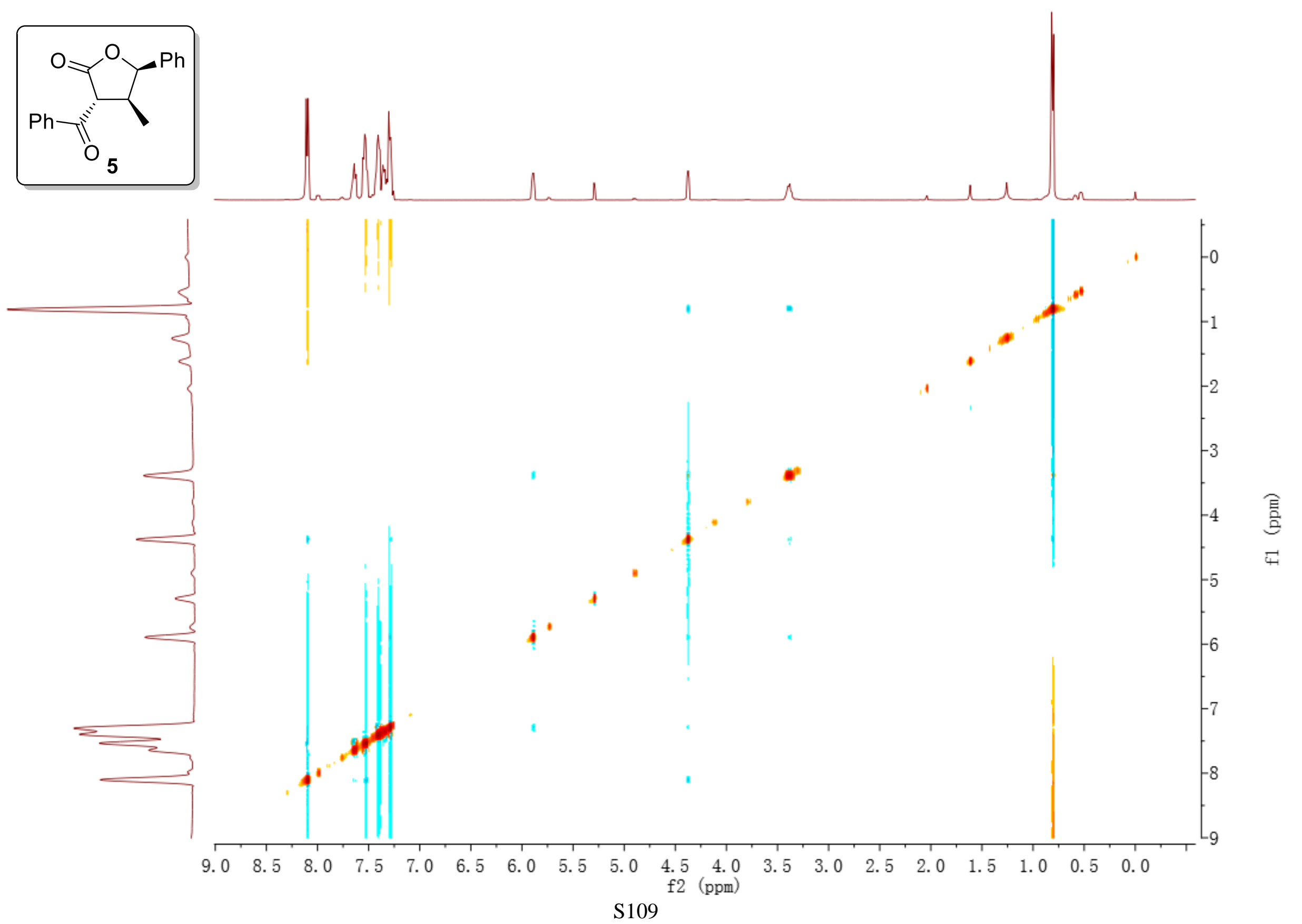

